\title{
NEUROINFLAMMATORY MECHANISMS OF PARKINSON'S
}

\section{DISEASE}

\author{
by \\ Emily Mangano \\ A thesis submitted to \\ the Faculty of Graduate and Postdoctoral Affairs \\ in partial fulfillment of the requirements for the degree of \\ Doctor of Philosophy \\ in \\ Neuroscience
}

\author{
Carleton University \\ Ottawa, Canada
}

(C)2011 Emily Mangano 
Library and Archives

Canada

Published Heritage Branch

395 Wellington Street Ottawa ON K1A ON4 Canada
Bibliothèque et

Archives Canada

Direction du

Patrimoine de l'édition

395 , rue Wellington

Ottawa ON K1A ON4

Canada
Your file Votre référence
ISBN: $978-0-494-79626-9$
Our file Notre référence
ISBN: $978-0-494-79626-9$
NOTICE:

The author has granted a nonexclusive license allowing Library and Archives Canada to reproduce, publish, archive, preserve, conserve, communicate to the public by telecommunication or on the Internet, loan, distribute and sell theses worldwide, for commercial or noncommercial purposes, in microform, paper, electronic and/or any other formats.

The author retains copyright ownership and moral rights in this thesis. Neither the thesis nor substantial extracts from it may be printed or otherwise reproduced without the author's permission.
AVIS:

L'auteur a accordé une licence non exclusive permettant à la Bibliothèque et Archives Canada de reproduire, publier, archiver, sauvegarder, conserver, transmettre au public par télécommunication ou par l'Internet, prêter, distribuer et vendre des thèses partout dans le monde, à des fins commerciales ou autres, sur support microforme, papier, électronique et/ou autres formats.

L'auteur conserve la propriété du droit d'auteur et des droits moraux qui protège cette thèse. $\mathrm{Ni}$ la thèse ni des extraits substantiels de celle-ci ne doivent être imprimés ou autrement reproduits sans son autorisation.
In compliance with the Canadian Privacy Act some supporting forms may have been removed from this thesis.

While these forms may be included in the document page count, their removal does not represent any loss of content from the thesis.
Conformément à la loi canadienne sur la protection de la vie privée, quelques formulaires secondaires ont été enlevés de cette thèse.

Bien que ces formulaires aient inclus dans la pagination, il n'y aura aucun contenu manquant.

\section{Canadä}




\begin{abstract}
Parkinson's disease (PD) has been linked to chronic exposure to environmental (e.g. heavy metals and pesticides) and immunological toxins. In particular, epidemiological studies show an increased prevalence of PD associated with exposure to pesticides; particularly, the herbicide, paraquat. Moreover, chronic exposure to paraquat has been reported to induce a loss of dopamine (DA) neurons within the substantia nigra pars compacta $(\mathrm{SNc})$ of rodents (as occurs with $\mathrm{PD}$ ). Accumulating evidence also indicates that chronic neuroinflammation (i.e. microglia-dependent inflammatory and oxidative processes) may mediate and/or enhance the neuropathological effects of environmental toxins. Hence, the following dissertation sought to assess the involvement of neuroinflammatory cascades in the neurodegenerative affects of paraquat in mice.
\end{abstract}

We presently demonstrated that pre-treatment with an immunological insult [lipopolysaccharide (LPS)] promoted neuroinflammatory cascades that sensitized DA neurons to the impact of subsequent paraquat exposure. However, this effect was dependent upon the timing between exposure to the two insults and the morphological state of microglia. Our current findings also suggested an important role for the proinflammatory cytokine, interferon-gamma (IFN- $\gamma$ ), in mediating the impact of paraquat upon DA neurons, and again, microglial cell alterations were related to the neurodegenerative cascade. Specifically, genetic ablation of IFN- $\gamma$ prevented the paraquat-induced activation of microglial and subsequent elevation of pro-inflammatory cytokines, inflammatory signalling factors and pro-oxidative factors, as well as the suppression of SNc trophic factors. 
Finally, administration of the trophic cytokine granulocyte macrophage colony stimulating factor (GM-CSF) treatment had neuroprotective consequences in LPS primed mice that also received paraquat exposure. Importantly, this trophic cytokine was able to impart such protective effects when administered either centrally or peripherally. GMCSF administration reversed the paraquat induced reduction of brain derived neurotrophic factor (BDNF) and was associated with a modulation of microglial state. In summary, the present dissertation supports the hypothesis that a pre-existing neuroinflammatory state may enhance the adverse consequences of later toxin exposure(s). We also demonstrate that IFN- $\gamma$ is a critical mediator of SNc neuron loss following toxin exposure and likely involved in the orchestration of microglial-dependent inflammatory and oxidative processes. Conversely, we provide evidence that GM-CSF can inhibit the neurodegenerative process following toxin exposure. Ultimately, these data should have important implications for understanding how environmental events can affect DA neurons and contribute to the development of novel strategies for treating PD. 


\section{Acknowledgements}

I would first like to thank my supervisor Shawn Hayley for his guidance and support throughout my graduate degrees. Shawn, I am indebted to you and I can't overstate how much I appreciate everything you have done for me. Your enthusiasm for research has always been an inspiration for me and I am grateful for your patience over the years and the efforts you have taken to help me become a better scientist. I am also grateful to advice I have received from my committee members, Dr. Anisman, Dr. Abizaid, Dr. DeRosa, and Dr. Bennett for their guidance and support.

Over the years I have been very fortunate to work alongside many great individuals who have taught me many of the techniques preformed in the following dissertation. I am grateful to guidance I received from Teresa Fortin for her assistance with developing and troubleshooting countless immunohistochemical procedures, Marzena Sieczkos for all her guidance in teaching me various in vivo procedures, Katie Cleland for taking the time to teach me molecular techniques. I am also extremely grateful to the many members of our team in Shawn Hayley's lab whose technical expertise I am indebted to. These include Geoffrey Crowe, Eric Nelson, Sarah Peters, Remmick So and Cheri Bethune. I would also like to take a moment to thank Darcy Litteljohn with whom I began my graduate studies with; over the years our collaboration has led to many publications.

Finally, this thesis could not have been completed without the support and love I have received from Dr. Marcelo Rocha, and my family. Special thanks must also go to Natasha Montemurro for taking the time to listen to all of my research talks I had to prepare over the years. Thank you for believing in every step of the way! 
This thesis is based on the following papers, which will be referred to in the text by

their roman numerals. Each paper will represent a separate chapter in the thesis.

I. Mangano E., Litteljohn D., So R., Nelson E., Peters S., Bethune C., Bobyn J, Hayley S. 2010. Interferon- $\gamma$ plays a crucial role in paraquat-induced neurodegeneration involving oxidative and pro-inflammatory pathways. Neurobiology of Aging, submitted.

II. Mangano, E.N. and Hayley S., 2009. Inflammatory priming of the substantia nigra influences the impact of later paraquat exposure: Neuroimmune sensitization of neurodegeneration. Neurobiology of Aging. 30, 1361-78.

III. Mangano, E.N., Peters S, Litteljohn D, So R, Bethune C, Bobyn J, Clarke M, Hayley S. 2010. Granulocyte macrophage-colony stimulating factor protects against substantia nigra dopaminergic cell loss in an environmental toxin model of Parkinson's disease. Neurobiology of disease, submitted. 


\section{Table of Contents}

Title page

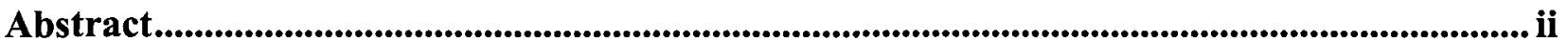

Acknowledgements .............................................................................................................................. iv

List of Abbreviations ............................................................................................................................. xi

List of Tables ............................................................................................................................................. xii

List of Figures..................................................................................................................................................... xiii

\section{Chapter 1}

General Introduction to the Neuroinflammatory Mechanisms of Parkinson's disease ......... 1

Background ...................................................................................................................................... 2

Toxin based animal models of PD ............................................................................. 8

Pesticides and Parkinson's disease …….................................................. 11

Immune toxins and Parkinson's disease ................................................. 15

Peripheral and central inflammation in Parkinson's disease ......................................... 17

Blood brain barrier and its importance in Parkinson's disease.

Entry of peripheral immune cells into the CNS: Role in Parkinson's disease pathology....

Neuroinflammatory mediated neurodegeneration: Role for microglia

Oxidative stress in PD-pathology: Role for pro-inflammatory cytokines 29

The role of pro-inflammatory cytokines and their downstream signalling

factors in Parkinson's disease

Interleukins and tumor necrosis factor- $\alpha$ in Parkinson's disease 33

Emerging evidence for a role of IFN- $\gamma$ signalling in Parkinson's disease 37

Therapeutics of Parkinson's disease: past, current and future...................................... 40

Research Objectives ................................................................................................................................ 46

The aims of this dissertation sought to assess.......................................................................... 47

\section{Chapter 2}

Interferon- $\gamma$ plays a crucial role in paraquat-induced neurodegeneration involving oxidative and pro-inflammatory pathways. 


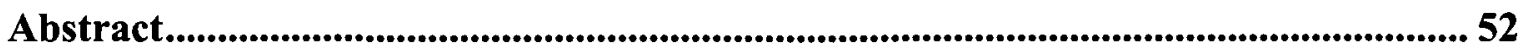

Introduction .......................................................................................................................... 53

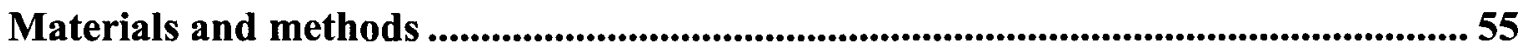

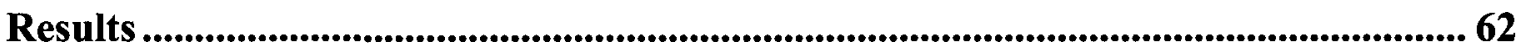

IFN- $\gamma$ deficiency prevented dopamine neuron loss following paraquat

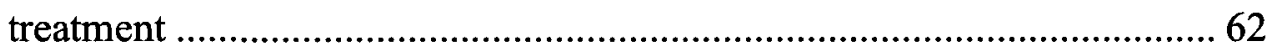

Accumulation of paraquat within the brain .............................................. 63

Figure 2.1 IFN- $\gamma$ KO mice were resistant to paraquat toxicity................ 64

Paraquat increased morphological signs of microglial reactivity and proinflammatory cytokine expression in wild-type but not IFN- $\gamma$ null mice. 65

Figure 2.2 IFN- $\gamma$ deficiency prevented paraquat induced activation of microglia.

IFN- $\gamma$ deficiency attenuated NADPH oxidase activation following paraquat exposure

Figure 2.3 Paraquat elevated the expression of several NADPH oxidase subunits within the SNc of wild type (WT) but not IFN- $\gamma$ knockout (KO) mice

Paraquat-induced changes in MAP kinase expression were prevented by IFN- $\gamma$ knockout

Paraquat provoked time- and- IFN- $\gamma$-dependent variations of inflammatory transcription factors and their downstream enzymes.

Figure 2.4 Paraquat-induced elevations of SNc mRNA of the MAP kinases, JNK3 and p38, were absent in IFN- $\gamma$ deficient mice.

Figure 2.5 Paraquat-induced elevations in the transcription factors, STAT1 and NFkB, as well as the inducible enzymes, iNOS and COX-2 are modulated by IFN $-\gamma$ deficiency.

Paraquat time-dependently diminished CREB and BDNF levels within the SNc among wildtype but not IFN- $\gamma$ null mice.

Discussion.

Figure 2.6 Paraquat-induced reductions of SNc mRNA for CREB and $\mathrm{BDNF}$ in the SNc are absent in IFN- $\gamma$ deficient mice.

Figure 2.7 Model of potential role of IFN- $\gamma$ on the pathways to pathology following paraquat exposure. 


\section{Chapter 3}

Inflammatory priming of the substantia nigra influences the impact of later paraquat exposure: Neuroimmune sensitization of neurodegeneration

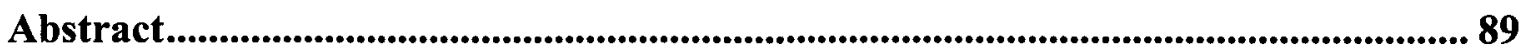

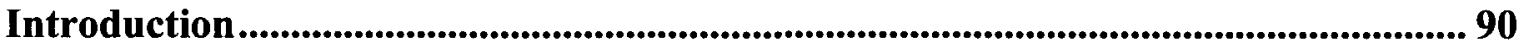

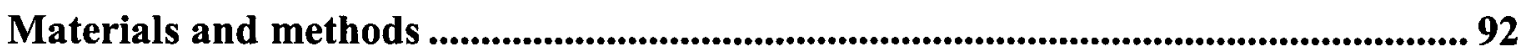

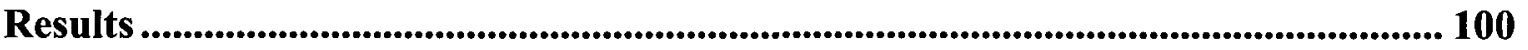

Experiment 1: LPS induced time-dependent SNc microglial activation 100 Infiltration of peripheral immune cells: CD3, CD11b and LMP 2 and 7 immunoreactivity at the meninges .................................................. 101

Figure 3.1 Intra-nigral LPS induced time-dependent changes in SNc microglia immunoproteasome expression. .................................. 102

Central and peripheral cytokine alterations following LPS ................. 104

Figure 3.2 Intra-nigral infusion of LPS provoked time-dependent elevation of CD3, LMP-2 and CD1 1b labelling at the meninges.......... 105

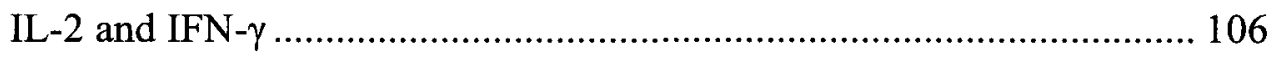

Experiment 2: LPS sensitized or de-sensitized DA neurons to paraquat depending upon timing of exposure................................................. 107

Figure 3.3 Intra-SNc LPS infusion provoked marked time-dependent elevations in several pro-inflammatory cytokines. ........................... 108

Figure 3.4 LPS time-dependently affected the sensitivity of SNc dopaminergic neurons to the effects of paraquat.

Inflammatory SNc glial changes that accompany the LPS-paraquat provoked neurodegeneration.

Figure 3.5 Intra-SNc infusion of LPS and systemic paraquat timedependently affected striatal dopaminergic terminal.

Figure 3.6 Intra-SNc infusion of LPS and systemic paraquat timedependently affected glial expression in the SNc.

Striatal glial and FGF changes accompany the LPS-paraquat treatments.

LPS and paraquat treatments provoked behavioural impairment

Figure 3.7 A 7-day delay between LPS and paraquat modulated glial response within the striatum to promote trophic support.

Figure 3.8 Toxin induced behavioral changes. 
Discussion.

Sensitization of neurodegenerative effects of LPS and paraquat (2 days)

Sensitized and de-sensitized glial responses associated with LPS and paraquat.

Cytokine variations provoked by LPS pre-treatment.............................. 126

LPS pre-treatment diminishes the impact of paraquat 7 days later

Conclusions

\section{Chapter 4}

Granulocyte macrophage-colony stimulating factor protects against substantia nigra dopaminergic cell loss in an environmental toxin model of Parkinson's disease...... 130

Abstract......................................................................................................................... 131

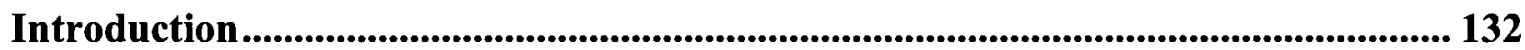

Materials and methods ............................................................................................................ 134

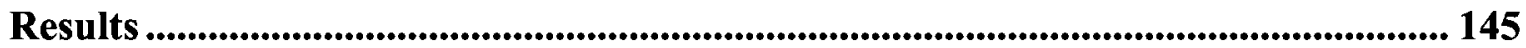

Experiment 1: Supra-nigral injection of GM-CSF or GDNF attenuated LPS-paraquat induced degeneration of SNc dopamine neurons and striatal terminals.

Figure 4.1 Central administration of GM-CSF and GDNF protected SNc neurons

Figure 4.2 Central administration of GM-CSF and GDNF attenuated the impact of LPS and paraquat upon striatal dopaminergic terminals.

Supra-nigral injection of GM-CSF influenced LPS and paraquat induced glial changes in the SNc.

Supra-nigral infusion of GM-CSF modified the paraquat induced reduction of hippocampal BDNF.

Experiment 2: Systemic administration of GM-CSF had neuroprotective consequences.

Figure 4.3 Central GDNF and GM-CSF modulated the impact of LPS and paraquat upon $\mathrm{SNc}$ astrocytes and microglia.

Figure 4.4 Central administration of GM-CSF influenced the effects of paraquat on hippocampal BDNF expression. 
Figure 4.5 Systemic GM-CSF treatment protected nigrostriatal neurons (both SNc soma and striatal terminals) from LPS and paraquat induced dopaminergic cell death

Experiment 3: GM-CSF neuroprotection was associated with normalized BDNF but not Bcl-2 or Bax levels.

Figure 4.6 Systemic GM-CSF administration modulated the effects of LPS and paraquat upon SNc astrocytes and microglia.

Discussion.

Figure 4.7 GM-CSF restored hippocampal BDNF expression in midbrain cultures exposed to paraquat.

\section{Chapter 5}

General Discussion

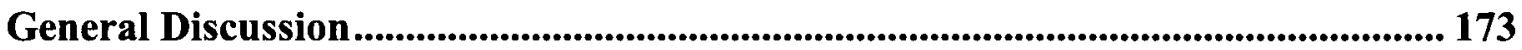

Paraquat and clinical aspects of Parkinson's disease .......................................... 175

Parkinson's disease and inflammation

Microglia are primary mediators of nigrostriatal damage

Cytokines and nigrostriatal degeneration: IL-1 $\beta$ and TNF- $\alpha$

Cytokines and nigrostriatal degeneration: IFN- $\gamma$

Anti-inflammatory and trophic cytokines protect nigrostriatal neurons. 186

Conclusions

Appendix A 


\section{List of Abbreviations}

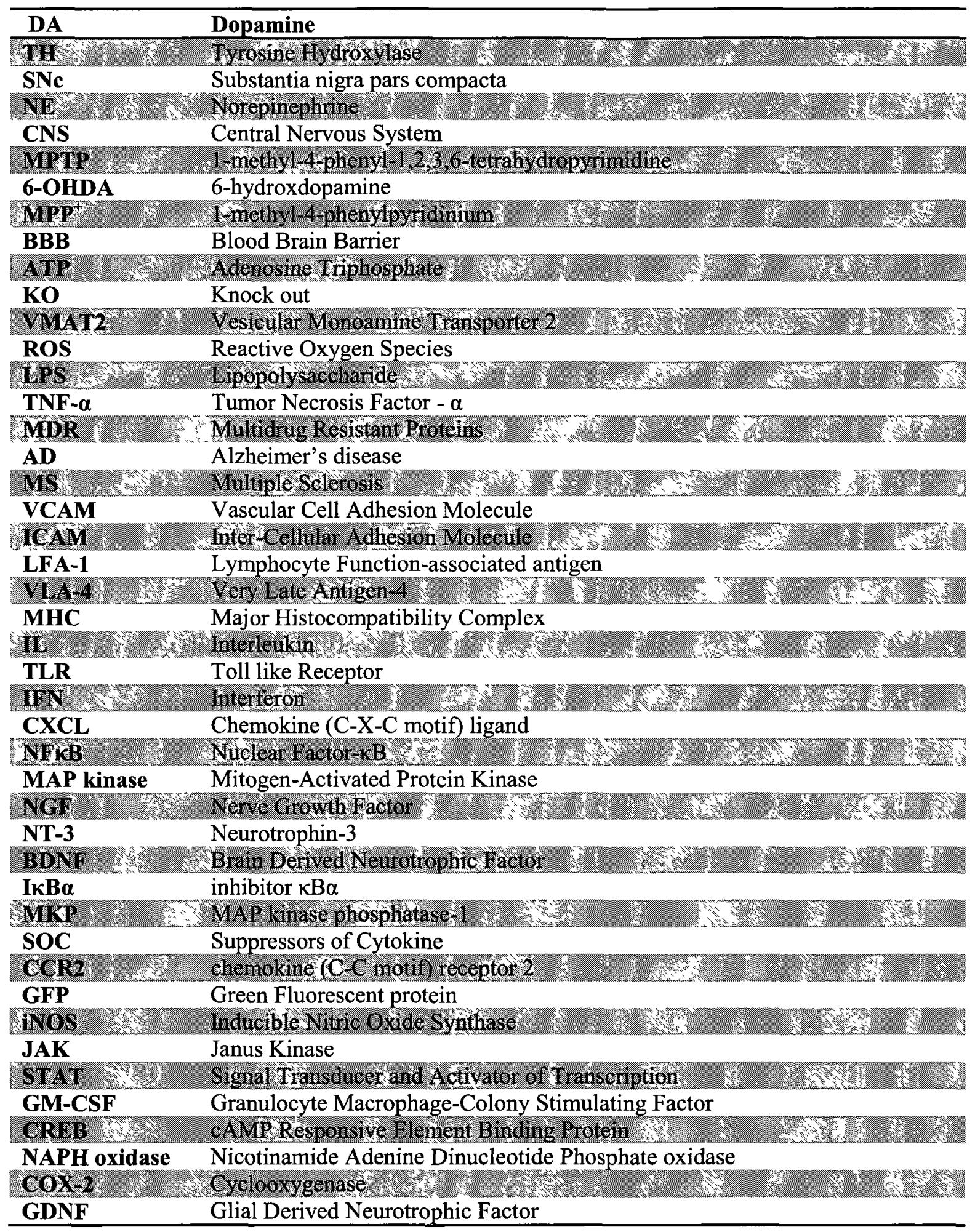


Proinflammatory cytokines and Parkinson's disease, xii

\section{List of Tables}

I. Table 2.1 SA Bioscience Gene List 


\section{List of Figures}

Figure 1.1 Anatomy of the Neurovascular Unit.

Figure 1.2 Leukocyte extravasation from bloodstream into the CNS.

Box 1.1 Apoptotic cell death in PD-like pathology.

Figure 2.1 IFN- $\gamma$ KO mice were resistant to paraquat toxicity.

Figure 2.2 IFN- $\gamma$ deficiency prevented paraquat induced activation of microglia.

Figure 2.3 Paraquat elevated the expression of several NADPH oxidase subunits within the SNc of wild type (WT) but not IFN- $\gamma$ knockout (KO) mice.

Figure 2.4 Paraquat-induced elevations in the transcription factors, STAT1 and NFkB, as well as the inducible enzymes, iNOS and COX-2 are modulated by IFN- $\gamma$ deficiency.

Figure 2.5 Paraquat-induced elevations of SNc mRNA of the MAP kinases, JNK3 and p38, were absent in IFN- $\gamma$ deficient mice.

Figure 2.6 Paraquat-induced reductions of SNc mRNA for CREB and BDNF in the SNc are absent in IFN- $\gamma$ deficient mice.

Figure 2.7 Model of potential role of IFN- $\gamma$ on the pathways to pathology following paraquat exposure.

Figure 3.1 Intra-nigral LPS induced time-dependent changes in SNc microglia immunoproteasome expression.

Figure 3.2 Intra-nigral infusion of LPS provoked time-dependent elevation of CD3, LMP-2 and CD11b labelling at the meninges.

Figure 3.3 Intra-SNc LPS infusion provoked marked time-dependent elevations in several pro-inflammatory cytokines.

Figure 3.4 LPS time-dependently affected the sensitivity of SNc dopaminergic neurons to the effects of paraquat.

Figure 3.5 Intra-SNc infusion of LPS and systemic paraquat time-dependently affected striatal dopaminergic terminal. 
Figure 3.6 Intra-SNc infusion of LPS and systemic paraquat time-dependently affected glial expression in the SNc.

Figure 3.7 A 7-day delay between LPS and paraquat modulated glial response within the striatum to promote trophic support.

Figure 3.8 Toxin induced behavioural changes.

Figure 4.1 Central administration of GM-CSF and GDNF protected SNc neurons.

Figure 4.2 Central administration of GM-CSF and GDNF attenuated the impact of LPS and paraquat upon striatal dopaminergic terminals.

Figure 4.3 Central GDNF and GM-CSF modulated the impact of LPS and paraquat upon $\mathrm{SNc}$ astrocytes and microglia.

Figure 4.4 Central administration of GM-CSF influenced the effects of paraquat on hippocampal BDNF expression.

Figure 4.5 Systemic GM-CSF treatment protected nigrostriatal neurons (both SNc soma and striatal terminals) from LPS and paraquat induced dopaminergic cell death.

Figure 4.6 Systemic GM-CSF administration modulated the effects of LPS and paraquat upon $\mathrm{SNc}$ astrocytes and microglia.

Figure 4.7 GM-CSF restored hippocampal BDNF expression in midbrain cultures exposed to paraquat.

Figure 6.1 Central administration of GM-CSF elevated Erk1/2 within the SNc following LPS and paraquat. 
Proinflammatory cytokines and Parkinson's disease, 1

\section{Chapter 1}

General Introduction to the Neuroinflammatory Mechanisms of Parkinson's disease 


\section{Background}

Parkinson's disease (PD) is the second most common neurodegenerative disease after Alzheimer's (Nagatsu and Sawada, 2005). Less than 10\% of all PD cases are caused by a known genetic mutation and the remaining cases are of unknown origin. Idiopathic PD affects approximately $1 \%$ of the population over the age of 65 , with the first signs of motor impairments becoming evident around 60 years of age (Tanner and Goldman, 1996). A recent large scale twin study was conducted in order to determine the genetic and the environmental risks involved in both types of PD. Not surprisingly, this study found that environmental risk factors do not play an important role in familial PD, whereas the incidence of idiopathic PD was correlated with exposure to environmental toxins found in certain areas, such as agricultural centers and industrial manufacturing plants (Landrigan et al., 2005; Logroscino, 2005; Nagatsu and Sawada, 2005).

The clinical signs of the disease stem from a dysregulation of the basal ganglia motor loop. Essentially, the major input area of the basal ganglia, the caudate-putamen nuclei (collectively referred to as the striatum in rodents) receives excitatory projections from most cortical areas, as well as midbrain dopamine (DA) producing neurons of the substantia nigra pars compacta (SNc). Degeneration of the dopaminergic neurons in the SNc represents the primary pathological hallmark of PD. The motoric deficits will appear following approximately $50 \%$ loss of $\mathrm{SNc}$ DA neurons, which corresponds to approximately $80 \%$ reduction of DA being released into the striatum (Toulouse and Sullivan, 2008). This neuronal loss is associated with a series of hallmark motor deficits including bradykinesia (slowness of movement), muscular rigidity (stiffness of limbs), postural instabilities and gait abnormalities (Blandini et al., 2000). As the disease progresses more of the nigrostriatal region will degenerate, which corresponds 
exaggeration and worsening of the symptoms (Toulouse and Sullivan, 2008). Neuronal loss is not isolated to the nigrostriatal pathway, in fact, post-mortem analysis of PD brains reveal a substantial loss of norepinephrine (NE) producing neurons in the locus coeruleus along with the loss of neocortical and hippocampal cells (Scatton et al., 1982). In addition to the nigrostriatal degeneration, a definitive PD diagnosis requires the presence of a significant number of Lewy bodies (Schulz-Schaeffer, 2010). Lewy Bodies are insoluble protein aggregates forming fibrils, composed mainly (but not exclusively) of the protein $\alpha$-synuclein (Rochet et al., 2004).

The clinical symptoms of PD ultimately arise from dysregulation of the basal ganglia motor circuitry. Although the SNc is technically not a part of the basal ganglia, its DA projections do strongly innervate post-synaptic D1 and D2 receptors in the striatum. Essentially, the striatum forms the major basal ganglia input and ligation of its DA receptors regulates the activity of downstream output nuclei (globus pallidus and substantia nigra pars reticulata) of this system. This, in turn, modulates thalamic output which is finally sent to the motor cortex (Blandini et al., 2000; DeLong and Wichmann, 2007). Activation of the D1 receptors promote signalling through the so called, 'direct' basal ganglia pathway, which essentially facilitates movement by relieving tonic inhibitory control of the thalamic projections to the motor cortex. In contrast, activation of the activation of the D2 receptors in the striatum results in stimulation of the 'indirect' pathway which leads to an overall inhibition of movement by facilitating inhibitory signals to the thalamus and motor cortex (Blandini et al., 2000; DeLong and Wichmann, 2007). In the case of $\mathrm{PD}$, disturbances of the direct pathway results in excessive inhibition of the major output nuclei; hence, leading to reduced thalamo-cortical activity. 
Likewise, disturbances of the indirect pathway that occur in PD result in diminished stimulation of the motor cortex (Blandini et al., 2000). Dysregulation of these two pathways ultimately results in the classical motor deficits associated with PD.

Although the mechanisms underlying dopaminergic cell death have yet to be fully elucidated, familial forms of the disease do account for a small number of cases (less than 5\%) (Betarbet et al., 2002; Kelada et al., 2006). Despite their extremely low penetrance, 6 causative genetic mutations have been linked with familial forms of PD (Morris, 2005). Specifically, genetic mutations coding for Leucine-rich repeat kinase 2 (LRRK-2), PARK3, and $\alpha$-synuclein account for the majority of autosomal dominant forms of Mendelian PD, while Parkin, PINK-1 and DJ-1 account for the autosomal recessive forms of the disease (Funayama and Hattori, 2007; Klein and Lohmann-Hedrich, 2007; Tan and Skipper, 2007; Thomas and Beal, 2007) Of course, there is the possibility that some yet to be identified genetic factors will emerge over time and explain a larger proportion of PD cases. However, it seems more likely that a combination of an intrinsic genetic vulnerability may act in a synergistic manner with environmental exposures to jointly influence the emergence of PD pathology.

Besides genetic alterations being of potential importance in the provocation of PD, accumulating evidence also points to environmental factors being involved. Specifically, epidemiological reports revealed a higher incidence of PD in areas with prominent agro-chemical usage or industrial areas with high levels of organic and heavy metal pollutants (Abbott et al., 2003; Baldi et al., 2003; Dick et al., 2007; Gorell et al., 2004; Hunot et al., 2004; Sherer et al., 2002a; Tanner and Goldman, 1996) Although there has yet to be agreement on any particular naturally occurring toxin that maybe 
responsible for $\mathrm{PD}$, the pesticides rotenone and paraquat, as well as a number of heavy metals, including manganese, iron and lead have been implicated in the progression of PD-pathology (Peng et al., 2007; Priyadarshi et al., 2001; Ritz and Yu, 2000; Tanner and Goldman, 1996). Indeed, case-control studies and meta-analyses have unveiled significant correlations between PD and prolonged occupational exposures to pesticides and heavy metals (Abbott et al., 2003; Gorell et al., 2004; Priyadarshi et al., 2001). In particular, the herbicide, paraquat, has emerged as a pesticide highly correlated with PD. Indeed, a Taiwanese cohort study found that exposure to paraquat had a greater association with PD in comparison to other pesticides (Liou et al., 1997). As will be discussed more fully in subsequent sections, paraquat can promote pathological features resembling PD-like syndrome in rodents, however, its long-term histopathological consequences in humans are presently unknown.

In spite of the overwhelming data discussed in this thesis supporting a role for pesticides in the provocation of $\mathrm{PD}$, there is very little evidence to support any individual pesticide alone as a causative factor for the onset of the disease (Brown et al., 2006) and there are even a few epidemiological reports that have failed to find a link between pesticide exposure and PD (Costello et al., 2009). This inconsistency may be owing to several factors including methodological limitations (relying on self-reports), which can result in biases. To overcome such limitations, one recent study attempted to assess the long term effects of paraquat and the fungicide maneb using a new evidence based model, which allowed the researchers to more accurately determine exposure rates to these pesticides (and eliminate self-report biases) in a rural Californian community (Costello et al., 2009). Interestingly, this is the first epidemiological report that clearly 
identified paraquat and maneb to be risk factors for developing PD. Furthermore, they also demonstrate that multiple toxin exposures over a person's lifetime can increase the chances for developing PD and the timing (specifically early life exposure) to these pesticides will increased the likelihood of developing PD later in life. Hence, it has become clear that environmental toxins (in particular paraquat) can influence the onset of PD and as will be discussed shortly, may even interact too synergistically with other insults to increase the risk of disease.

In addition to environmental agents, immunological insults of viral or bacterial origin may also contribute to the development of PD. This may be especially true for patients that develop a PD-like syndrome following encephalitis (Jang et al., 2008). It has been postulated that a subset of PD patients exposed to an infection prenatally or early in life may bring about the disease later in life (Takahashi and Yamada, 1999). For instance, documented cases of a PD-like syndrome were reported years following von Economo encephalitis and the influenza epidemic of 1918 (Casals et al., 1998; Dickman, 2001). Moreover, rodents exposed to the Japanese encephalitis virus undergo a few of the pathological features associated with $\mathrm{PD}$, including reduced number of DA neurons and marked gliosis coupled with PD-like behavioural symptoms such as bradykinesia (Ogata et al., 1997).

In addition to viruses, prenatal or early life exposure to a bacterial pathogen may also be implicated in the disease onset. Recent animal studies have revealed that rats prenatally exposed to LPS displayed substantially less DA neurons within the SNc (Carvey et al., 2003). Along these lines, rodents exposed to low concentrations of pesticides early in life were more susceptible to DA neurotoxins later in life 
(Thiruchelvam et al., 2000b). Therefore, it remains possible that early life exposure to immunological toxins may provoke a modest neuroinflammatory response that over a prolong period of time can initiate neurodegeneration or perhaps make the DA neurons more vulnerable to neurotoxic insults later in life (Hayley and Anisman, 2005).

Nonetheless, regardless of the type of toxin (immunological or environmental), the chronicity, concentration and/or pattern of exposure may ultimately be important in shaping the progression of PD. Although acute toxin exposure is likely handled by detoxification systems without damage to nearby tissue, repeated exposure may disturb central nervous system (CNS) homeostasis, resulting in maladaptive responses (e.g. prolonged activation of neuroinflammatory and oxidative cascades) and culminate in neuronal degeneration.

The present thesis will focus primarily upon the contribution of immune factors in the degeneration of SNc DA neurons using an environmental toxin-based model of PD. Specifically, the involvement of pro- and anti-inflammatory cytokines, coupled with growth factors and changes in the brain's own specialized immune cells (namely microglia) will be assessed in the following chapters. Briefly, the thesis will discuss the evidence indicating that chronically "hyper-reactive" microglia and associated cytokines (in particular interferon- $\gamma$ ) and oxidative factors may play a provocative role in PDpathology. In contrast, as we demonstrate in Chapter 3, a cytokine with growth factor properties, granulocyte macrophage-colony stimulating factor (GM-CSF) can have neuroprotective effects, owing to its immune modulatory and trophic effects. Overall, we posit that chronic toxin exposure (e.g. the herbicide, paraquat) can promote a state of chronic microglial activation, which in turn causes deleterious consequences in line with 
those observed in PD. To this end, finding novel means to counteract this neuroinflammatory response might ultimately interrupt the pro-death processes and promote recovery.

\section{Toxin based animal models of PD}

Three interrelated mechanisms are currently believed to be fundamental to the neurodegenerative effects caused by the classical DA toxins 1-methyl-4-phenyl-1,2,3,6tetrahydropyrimidine (MPTP) and 6-hydroxdopamine (6-OHDA), as well as the pesticides rotenone and paraquat: (1) inhibition of complex I of the mitochondrial respiratory chain, (2) activation of oxidative/apoptotic stress factors and (3) provocation of neuroinflammatory cascades. Importantly, various combinations of these three mechanisms likely contribute to the neuronal demise following differing toxin exposures. As will be discussed shortly, it is also critical to note that these toxins differentially affect pro-death processes. For instance, while MPTP and rotenone markedly inhibits mitochondrial complex I, paraquat is not believed to have such an effect. However, collectively all of the above mentioned toxins are known to induce signs of oxidative distress and pro-inflammatory cascades, which of course, is a main focus of the present thesis.

6-OHDA and MPTP are the most commonly employed toxins used in rodents and primates to elicit PD-like pathology (Bergman et al., 1998; Blum et al., 2001; Bove et al., 2005; Carrasco et al., 2005; German et al., 1996; Quintero et al., 2006; Salach et al., 1984). 6-OHDA is a hydroxylated analogue of DA, which is capable of producing severe peripheral and central lesions. Given that this DA toxin cannot penetrate the blood brain barrier (BBB), it must be administered either directly into the striatum, $\mathrm{SNc}$ or ascending 
medial forebrain bundle. The exact mechanism by which 6-OHDA is taken up by DA neurons is currently unknown; however, it is likely that the toxin is taken-up by the dopamine transporter. It has been shown that cytosolic accumulation of 6-OHDA will generate cytotoxic metabolites (e.g. quinones, superoxide radicals, hydrogen peroxide and the hydroxyl radicals) in a manner similar to endogenous dopaminergic autooxidation upon encountering monoamine oxidase (Blum et al., 2001; Gomez-Lazaro et al., 2008; Rodriguez-Pallares et al., 2007). In addition to 6-OHDA's ability to generate free radicals, this toxin has also been associated with an up-regulation of the proapoptotic factor Bax. See Box 1 for a detailed description of apoptosis in PD. Essentially, 6-OHDA will promote translocation of Bax to the outer mitochondrial membrane, resulting in the release of cytochrome $\mathrm{C}$ in to the cytoplasm and ultimately, apoptosis (Gomez-Lazaro et al., 2008; Yang and Tiffany-Castiglioni, 2008).

There are clear limitations to using 6-OHDA as an animal model of PD; for one, the requirement of administration of 6-OHDA directly into the nigrostriatal pathway. Secondly and most importantly, the time course for 6-OHDA induced DA cell death is very rapid. This clearly is not consistent with the very slow progressive nature of pathology normally observed in PD (Bove et al., 2005). Lastly, 6-OHDA is unable to cause the formation of Lewy body inclusions despite causing massive depletion of DA in the nigrostriatal region.

Some of the drawbacks associated with the 6-OHDA toxin model can be addressed by using the meperidine derivative, MPTP. This toxin is highly lipophilic and easily crosses the $\mathrm{BBB}$ preferentially targeting several midbrain DA neuronal populations, namely the A8 (retrorubral nucleus), A9 (substantia nigra), and A10 (ventral 
tegmental area) regions (German et al., 1996). Systemic exposure to MPTP has been used over the past two and a half decades to provoke $\mathrm{SNc}$ dopaminergic degeneration coupled with depletion of striatal dopamine in mice and primates (Bergman et al., 1998; Boulet et al., 2008; Czlonkowska et al., 1996; Przedborski et al., 1996). Essentially, MPTP is metabolized by monoamine oxidase B and converted into its active metabolite, $\mathrm{MPP}^{+}$, which is taken up by the dopamine transporter and into dopaminergic terminals (Nicklas et al., 1985). Early studies with MPTP determined that $\mathrm{MPP}^{+}$is a particularly potent mitochondrial complex I inhibitor, eventually culminating in decreased adenosine triphosphate (ATP) levels, loss of mitochondrial membrane potential, faulty intracellular calcium buffering and free radical generation (Blum et al., 2001).

More recent studies have demonstrated that MPTP can also induce an inflammatory response that can be detrimental to DA neurons. Important for the present thesis, numerous studies have demonstrated that MPTP induces robust microglial activation and stimulation of inflammatory messengers (Barcia et al., 2004; Bolin et al., 2005; Czlonkowska et al., 1996; Du et al., 2001; Feng et al., 2003; Hayley et al., 2004a; Wu et al., 2002). Moreover, pharmacologically blocking the microglia response can prevent MPTP induced degeneration of DA neurons (Wu et al., 2002). Unlike 6-OHDA, non-human primate exposure to low doses of MPTP have been reported to cause a Parkinsonian-like syndrome that mimics many of the clinical attributes of PD, including bradykinesia and resting tremors (Bergman et al., 1998; Boulet et al., 2008; Mounayar et al., 2007). Somewhat perplexingly however, long-term evaluation of MPTP has revealed that some of the neurochemical (striatal DA levels) and behavioural deficits do normalize 
over time, probably resulting from compensatory mechanisms being eventually recruited (Boulet et al., 2008; Mounayar et al., 2007).

\section{Pesticides and Parkinson's disease}

Substantial epidemiological data and meta-analyses have revealed that geographical location and exposure to a range of environmental contaminants are possible contributors to PD-pathology (Abbott et al., 2003; Cicchetti et al., 2009; Dhillon et al., 2008; Dick et al., 2007; Tanner and Goldman, 1996). In particular, pesticide exposures have consistently shown to increase the risk of developing PD, and this has been further strengthened using animals studies (Gao et al., 2003a; Gupta et al., 2010; Manning-Bog et al., 2002; McCormack et al., 2002; Mount et al., 2007; Peng et al., 2007; Shimizu et al., 2003; Sindhu et al., 2005; Thiruchelvam et al., 2000a). As such, several animal models administering different pesticides, including rotenone or paraquat, have been shown to cause progressive loss of midbrain DA neurons and in some cases other brains regions, including the locus coeruleus (Chen et al., 2010; Hoglinger et al., 2003).

Rotenone is a naturally derived compound that is widely used as an insecticide in organic gardening and fishery management (Greenamyre et al., 2003). Interestingly, rotenone like MPTP, is a highly lipophilic compound that easily crosses the BBB and acts to inhibit mitochondrial complex 1 of the electron transport chain (Richardson et al., 2005) resulting in diminished ATP production and the production of pro-apoptotic factors. Despite the fact that rotenone inhibits mitochondrial complex 1 in many brain regions midbrain dopaminergic neurons appear to be the most sensitive, suggesting, that these neurons are intrinsically vulnerable (Sherer et al., 2003a). Moreover, free radical accumulation has been attributed to rotenone-induced dopaminergic damage and this was 
supported by data which demonstrated that pre-treatment with an anti-oxidant (e.g. alphatocopherol) attenuated DA loss in mice normally induced rotenone (Sherer et al., 2003b).

Selective vulnerability of DA neurons may stem, in part, from interactions of DA toxins with the vesicular monoamine transporter 2 (VMAT2), which is responsible for uptake of cytosolic DA (Caudle et al., 2007). As previously mentioned, 6-OHDA can cause abnormal accumulation of oxidative radicals and toxic DA-quinone products. Indeed, a dysfunctional VMAT2 will prevent cytosolic DA from being properly taken up into the vesicles, thus allowing cytosolic DA to auto-oxidize and produce these cytotoxic products. Indeed, in vitro analysis revealed that rotenone can disrupt VMAT2 functioning by promoting nitration of the transporter's tyrosine residues (Watabe and Nakaki, 2008). Similarly, DA neurons are more vulnerable to sub-threshold levels of rotenone in the presence of nitrative free radicals (Ahmadi et al., 2008).

In addition to promoting oxidative stress, rotenone can cause other histopathological features resembling PD that are not observed with 6-OHDA or MPTP. In particular, rotenone can cause the formation of $\alpha$-synuclein positive Lewy body inclusions (Betarbet et al., 2006b; Sherer et al., 2002b), which are believed to reflect disturbances of protein folding, among other pathological processes occurring in PD brains. However, like the more classical DA toxins, rotenone also induces and heightened microglial activation (Zhou et al., 2007), which is associated with the production of reactive oxygen species (ROS) and inflammatory mediators. As we will discuss shortly, numerous studies revealed that microglial released pro-inflammatory and oxidative factors are key mediators of DA cell death in PD. 
Similar to rotenone, the herbicide paraquat can also promote pathological features that are believed to resemble those in idiopathic PD. This herbicide can provoke histopathological and behavioural changes reminiscent to PD (Brooks et al., 1999; Cicchetti et al., 2005; Fernagut et al., 2007; Litteljohn et al., 2009; Litteljohn et al., 2008; Mangano and Hayley, 2009; Ossowska et al., 2005b; Thiruchelvam et al., 2000a; Thiruchelvam et al., 2003). In this regard, paraquat is the third most commonly used pesticide in the world. Thirteen countries (which are largely within the European Union) have recognized the dangerous side-effects of both direct and indirect exposure to paraquat and have thus banned the use of this herbicide; curiously, Canada and the United States are not included in this list (Cicchetti et al., 2005).

Paraquat is chemically similar to the active metabolite of MPTP, $\mathrm{MPP}^{+}$and can reliably provoke a progressive loss of nigrostriatal DA neurons (Andersen, 2003b; McCormack et al., 2002; Ossowska et al., 2005b; Peng et al., 2004; Peng et al., 2007; Purisai et al., 2007; Richardson et al., 2005; Somayajulu-Nitu et al., 2009; Yang et al., 2007; Yang and Tiffany-Castiglioni, 2008). Although the degree of neuronal loss induced by paraquat is less than that induced by MPTP $(\sim 30 \%$ versus $50 \%)$; like rotenone, paraquat can provoke the formation of Lewy body inclusions (Mak et al., 2010; Manning-Bog et al., 2003; McCormack et al., 2002).

In addition to these pathological hallmarks, paraquat can trigger behavioural disturbances reminiscent of PD. In particular, we have previously shown that mice chronically treated with paraquat experienced a loss of striatal DA coupled with reduced locomotor activity, which was evident by impaired vertical forelimb and hindlimb coordination ( $\mathrm{Li}$ et al., 2005; Litteljohn et al., 2009; Litteljohn et al., 2008). Similarly, 
aged mice ( 5 or 18 months) concomitantly exposed to paraquat and the fungicide maneb experienced robust motor deficits, which remained stable even 3 months following toxin exposure (Ossowska et al., 2005b; Thiruchelvam et al., 2003). In addition, paraquat treatment can also trigger non-motoric behavioural disturbances, including diminished performance on a forced swim test and open field (Chen et al., 2008; Litteljohn et al., 2009; Litteljohn et al., 2008), which is often taken to reflect affective disturbances. Presently, the underlying mechanism responsible for paraquat induced DA cell death remains unknown. However, our own work and that of others suggest that activation of neuroinflammatory cascades, along with the production of oxidative radicals plays an important role.

Like rotenone, paraquat has been shown to cross the BBB using a specific amino acid transporter (system L-carrier) and accumulate in the nigrostriatal pathway without compromising the integrity of the barrier (McCormack and Di Monte, 2003; Shimizu, 2001). Administration of the neutral amino acid L-valine (a competitive reuptake inhibitor) prior to paraquat treatment attenuated DA loss in the SNc by preventing paraquat from accumulating in the brain (McCormack and Di Monte, 2003). Upon entry into the brain, the mechanism by which paraquat elicits its neurotoxic effects appears to be different from that of MPTP and rotenone. Indeed, paraquat does not inhibit mitochondrial complex 1 of the electron transport chain; but rather it acts as a redox cycling agent, ultimately promoting free radical accumulation, coupled with neuroinflammation and mitochondrial dysfunction (McCormack et al., 2005; Richardson et al., 2005). Such effects are linked to the fact that paraquat has two positively charged 
ions allowing it to generate large quantities of ROS upon contact with molecular oxygen (Richardson et al., 2005).

\section{Immune toxins and Parkinson's disease}

The neurodegenerative effects of PD may be enhanced if superimposed with several risk factors including, advanced age, exposure to a previous toxin, as well as mutations of the $\alpha$-synuclein gene (Manning-Bog et al., 2003; Thiruchelvam et al., 2003). As will be discussed in detail in Chapter 2 of this thesis, priming the SNc with an immunological toxin (lipopolysaccharide; LPS) prior to paraquat exposure or concomitant exposure of paraquat with the fungicide maneb greatly augmented the degree of DA cell death in the SNc (from 30 to 50\% DA cell loss) (Mangano and Hayley, 2009; Purisai et al., 2007). Similarly, exposure to a sub-toxic dose of iron synergistically augmented the effects of paraquat on SNc DA neurons and this was related to both a reduction in number of available anti-oxidants and an increase in the pro-apoptotic transcription factor c-Jun N-terminal kinases (JNK). Accordingly, pretreatment with a synthetic superoxide dismutase/catalase mimetic, EUK-189 or pharmacological inhibition of JNK prevented DA cell death later caused by iron and paraquat (Peng et al., 2007).

It might be the case that insults which occur during development have delayed consequences on the nigrostriatal system only becoming evident with increased age and exposure to subsequent insults. Alternatively, a developmental insult may accelerate the normal loss of DA neurons across a person's life, whereby pathology only becomes evident if a critical threshold of DA loss has been surpassed. Indeed, studies assessing the long term affect of immune insults upon DA functioning demonstrated that early life 
exposure to challenges such as LPS, increased the vulnerability of developing PD-like pathology later in life. For instance, prenatal exposure to LPS at embryonic day 10.5 resulted in fewer overall number of SNc dopaminergic neurons in adult rats and elevated levels of the pro-inflammatory cytokine tumor necrosis factor (TNF- $\alpha$ ) in the striatum (Ling et al., 2002). In addition to the overall reduction in DA neurons, prenatal LPS exposure exacerbated the impact of later toxins including rotenone, 6-OHDA or supranigral injection of LPS. Specifically, re-exposure to a subsequent toxin resulted in greater microglial activation in the SNc as well as an up-regulation of TNF- $\alpha$ in the striatum (Ling et al., 2004a; Ling et al., 2006; Ling et al., 2004b).

Similar to the prenatal studies using LPS, early-life exposure to environmental toxins such as paraquat alone or when combined with maneb sensitized DA neurons further to the deleterious actions of subsequent nigrostriatal insults (Cory-Slechta et al., 2005). Specifically, exposure to paraquat or paraquat + maneb at postnatal days 5-19 enhanced the loss of SNc DA neurons and motor impairment when re-exposure to these insults occurred month's later (Thiruchelvam et al., 2005). It is conceivable that ongoing neuroinflammation following such challenges may sensitize neurons to the degenerative consequences of subsequent environmental insults.

As already mentioned, neuroinflammatory processes are likely fundamental for the oxidative damage provoked by DA toxins (Czlonkowska et al., 2002; KurkowskaJastrzebska et al., 1999a). In this respect, it is important to underscore that each of the environmental agents discussed thus far have been shown to induce signs of neuroinflammation. This is not surprising given that a primary role of inflammatory immunological functioning is to rid the body of such neurotoxins. Accordingly, it is our 
contention that excessive activation of central and peripheral immune factors (such as cytokines) stimulated by these challenges may contribute to neuronal tissue damage evident in PD.

\section{Peripheral and central inflammation in Parkinson's disease}

Prolonged activation of pro-inflammatory factors within the CNS is a common feature of most neurological conditions, including PD, Alzheimer's disease (AD), multiple sclerosis (MS), and cerebral stroke. Neuroinflammation is characterized by the presence of hyper-reactive microglia, often coupled with infiltration of adaptive cells such as T-lymphocytes (Re and Przedborski, 2006). It is well documented that once activated microglia are capable of inducing a self- self-sustaining pro-inflammatory state that can be detrimental to the CNS (Block et al., 2007). However, the primary initiator(s) of such a response in $\mathrm{PD}$ are not well understood. Speculation surrounding the possibility that an adaptive immune response, most notably infiltration of effector T-lymphocytes and macrophages, could initiate the central microglial response has begun to gain momentum in recent years. Yet, the traditional "neurocentric" view of PD has continued to favour a central location for the genesis of the toxic processes that take place in PD.

The innate immune system (predominantly macrophages, granulocytes, natural killer cells and microglia) acts as a first line of defence removing any unwanted antigens, while at the same time surveying for pathogens within the periphery. Upon detection of unwanted pathogens within the CNS, innate immune cells will recruit members of the adaptive immune system (i.e. effector $\mathrm{T}$ lymphocytes) by releasing pro-inflammatory cytokines and chemokines. Subsequently, resident immune cells (astrocytes and microglia) will up-regulate adhesion molecules on the BBB and promote CNS infiltration 
of peripheral immune factors see Figure 1.1. It is essential that this neuroinflammatory response remain tightly regulated otherwise there is the potential that an excessive number of peripheral immune cells can gain access to the CNS and promote the release of neurotoxic factors from resident glial cells. In the case of PD, it is unclear if neuroinflammation is primarily responsible for initiating neuronal death or rather if it is a secondary reaction activated in response to dead or dying DA neurons.

\section{Blood brain barrier and its importance in Parkinson's disease}

The CNS is protected from the periphery by a three layer encasing: the dural membrane (outer), the arachnoid membrane (middle) and the pial membrane (inner). A combination of vasculature (blood vessels and smooth muscle cells) and central components (consisting of endothelial cells, neurons and astrocytes) work together as a unit to nourish the brain; collectively this is referred to as the neurovascular unit, see Figure 1.2

Recent reports have demonstrated that the integrity of the $\mathrm{BBB}$ is disrupted in $\mathrm{PD}$ patients (Zlokovic, 2008). Essentially, the BBB monitors exchanges between the brain and periphery and prevents a range of potentially harmful substances (e.g. environmental toxins) from gaining access to the brain. In detail, the BBB consists of a unique monolayer of endothelial cells held together by tight junctions (Hawkins and Davis, 2005). At irregular intervals vascular cells called pericytes are embedded between the endothelial cells, which maintain the stability of the cerebral microvessels and modulate cerebral blood flow (Bell and Zlokovic, 2009; Dore-Duffy, 2008). Together with the endothelial cells, pericytes are enclosed by a basement membrane (also referred to as the basal lamina extracellular matrix) that connects with the astrocytic end-feet and nearby microglia. Permeability of the BBB may stem from a variety of complications including 
malformation of blood vessels, dysfunctional transporter mechanisms or incomplete formation of tight junctions. P-glycoprotein is one of many transporters located on the BBB involved in the transport of specific lipid-soluble molecules into the brain and has recently been implicated in degeneration of DA neurons (Dutheil et al., 2010; Westerlund et al., 2009).

Transport across the BBB against a concentration gradient requires facilitated transporters and thus ATP. Of all the transporters, P-glycoprotein was the first Multidrug Resistant protein identified; it is highly expressed on the luminal side of BBB where it transports a large number of xenobiotics from the brain back into the blood (Bernacki et al., 2008; Juliano and Ling, 1976; Terasaki and Hosoya, 1999). Interestingly, polymorphisms in the P-glycoprotein gene have been identified in PD patients (Westerlund et al., 2009). Paralleling these findings, chronic neuroinflammation induced by LPS can also influence the integrity of BBB by disrupting P-glycoprotein (Salkeni et al., 2009).

Very little is known about the effects of normal aging on the BBB; using brain imaging techniques few studies have demonstrated that the BBB becomes more permeable as a healthy brain ages (Farrall and Wardlaw, 2009). For instance, the xenobiotic transporter P-glycoprotein is significantly down-regulated in specific brain regions of aged humans (Bartels et al., 2009). Given that P-glycoprotein removes detoxified substrates from the brain any disruptions in this transporter can lead to a build up of toxic material within the brain. Upon analysis of PD patients, this transporter appears to be disrupted in during the later stages of the disease (Bartels et al., 2008a; Bartels et al., 2008b). Given that this impairment does not occur early during the disease 
suggests that any disruptions in P-glycoprotein may not be the initial cause of DA cell death. Nonetheless, it may further any ongoing degeneration preventing the removal of toxic by-products.

Furthermore, a dysfunctional BBB transport system could magnify the accumulation of neurotoxic material within the brain over time. For example, a combination of polymorphisms in the ABCB1 gene, which are associated with altered $\mathrm{P}$ glycoprotein function and occupational exposure to organochlorine insecticides were reported to interact and increase the risk of PD (Dutheil et al., 2010). This is in line with a recent report which showed that $\mathrm{P}$-glycoprotein participates in the extrusion of amyloid beta from brain parenchyma (Kuhnke et al., 2007; Westerlund et al., 2009). To this end, it remains possible that a disruption in P-glycoprotein (or any other BBB transporter) would increase brain exposure to neurotoxins normally excluded by these xenobiotic transporters.

Increased permeability of the BBB has been associated with both neuroprotective and pathological infiltration of peripheral immune cells (de Boer and Gaillard, 2006; Zlokovic 2008). Microglial cells localized close to the BBB are sensitive to changes in the brain homeostasis and can react by releasing factors that influence the permeability of the BBB. Upon recognizing any central disturbances microglia can induce the expression of cell-surface antigens and up-regulate cytokines and chemokines in an attempt to recruit innate and adaptive immune cells to an area of injury in order to re-establish homeostasis (Zlokovic 2008). 
Several reports have suggested that the pro-inflammatory cytokine, TNF- $\alpha$, can regulate the permeability of the BBB and extravasation of leukocytes into the CNS by promoting the expression of adhesion molecules [i.e. intracellular adhesion molecule-1 (ICAM-1)] on the endothelium (Dickstein et al., 2000; Dobbie et al., 1999; Wong and Dorovini-Zis, 1992). This is in line with post-mortem reports that have demonstrated an increase in TNF- $\alpha$ in the brains of PD patients (Mogi et al., 1994). However, the involvement of TNF- $\alpha$ in the regulation of BBB permeability has been contested by recent reports using transgenic mice lacking TNF- $\alpha$ receptors (Phares et al., 2007). We and others postulate that microglia-dependent release of TNF- $\alpha$ can affect the permeability of the BBB indirectly by activating other microglial-dependent processes. In particular, TNF- $\alpha$ can promote the accumulation of peroxynitrite indirectly, which can directly affect the permeability of the BBB (Hooper et al., 2000; Kean et al., 2000).

The pro-inflammatory cytokine, IFN- $\gamma$, which is also linked to PD pathology (Mount et al., 2007) can modulate the permeability of the BBB by stimulating the production of the highly toxic radical, peroxynitrite, by activating either eNOS directly on the endothelium or microglia-dependent processes (Gupta et al., 2010; Mander and Brown, 2005; Phares et a1., 2007). Despite that fact that the exact mechanism responsible for modulating the permeability of the BBB is not clear, a chronic state of inflammation can enhance long term permeability of the $\mathrm{BBB}$; and combined with chronic exposure to environmental toxins and a genetic vulnerability may render some individuals more susceptible to the impact of neurotoxins. 
It is also important to mention that systemic infections can also induce a central immune response. Indeed, various regions of the brain are not encased by BBB rather they are highly vascularized, allowing large molecules to diffuse into the CNS (Rivest, 2009). These regions are referred to as circumventricular organs and are located in various regions all around the brain, including organum vasculosum of the lamina terminalis, the subfornical organ, the median eminence and the area postrema (Rivest, 2009). Circulating immune cells (e.g. effector $T$ cells or macrophages) or pathogens $/$ toxins can easily enter in the brain parenchyma and induce a central immune response. Therefore, understanding how a state of chronic neuroinflammation induced either by microglia or the periphery can influence the integrity of the $\mathrm{BBB}$ may provide insight as to immune responses influence CNS-pathologies.

Entry of peripheral immune cells into the CNS: Role in Parkinson's disease pathology The inflammatory response within the CNS differs from peripheral mediated inflammation not only because of the BBB but also the absence of a true lymphatic drainage system, and minimal endogenous antigen presenting cells (Man et al., 2007). In non-pathological conditions, leukocyte populations (mainly $\mathrm{CD}^{+}$T-lymphocytes) routinely migrate into distinct CNS compartments. This process of extravasation (infiltration) is tightly controlled by chemokines, adhesion molecules and their respective receptors located predominantly at the post-capillaries venules, see Figure 1.1 (Butcher, 1991; Springer, 1994; Weninger and von Andrian, 2003). Essentially, effector Tlymphocytes migrate across tightly regulated areas of the CNS for immunosurveillance purposes, patrolling and searching for antigen presenting cells expressing corresponding antigen fragments. Despite the beneficial functions, leukocyte trafficking of $\mathrm{CD}^{+} \mathrm{T}$ - 
lymphocytes into the CNS may contribute to a leaky BBB by promoting the release of peroxynitrite from eNOS (Phares et al., 2007).

Microglial release of pro-inflammatory cytokines can act on the BBB endothelium and stimulate an up-regulation of the adhesion molecules: ICAM-1 and vascular cell adhesion protein-1 (VCAM-1) (de Boer and Gaillard, 2006; Neumann et al., 1998). This up-regulation can promote the recruitment and extravasation of circulating Tlymphocytes and monocytes that express their counter receptors, lymphocyte functionassociated antigen-1 (LFA-1) and very late antigen-4 (VLA-4), respectively (Neumann et al., 1998). It has been postulated that ICAM-1 and LFA-1 are essential for facilitating the extravasation of peripheral immune cells into the CNS. Indeed, ICAM-1 and its receptor, LFA-1 are over expressed in SNc PD patients; in addition, humans as well as non-human primates exposed to MPTP revealed intense ICAM-1 immunostaining in areas of extensive DA cell loss (Miklossy et al., 2006).

The role of the adaptive immune system ( $\mathrm{T}$ and $\mathrm{B}$ lymphocytes) in PD remains a controversial topic, despite the fact that post-mortem analysis of PD brains revealed extensive infiltration of $\mathrm{CD} 4^{+}$and $\mathrm{CD} 8^{+} \mathrm{T}$-lymphocytes (Brochard et al., 2009). These infiltrating immune cells, particularly $\mathrm{CD}^{+} \mathrm{T}$-lymphocytes can only cause an immune response following the interaction with an appropriate antigen-presenting cell (e.g. macrophages, dendritic cells and microglia). Prior to the interaction with an effector Tlymphocyte an antigen-presenting cell must first recognize a foreign pathogen, digest it, and then display fragments of the antigen on its own surface. Bound to this antigen fragment is a major histocompatibility complex (MHC) molecule that will attract the effector T-lymphocytes. Once a T-lymphocyte with the correct receptor fits the 'antigen- 
MHC complex', the T-lymphocyte will bind to it and stimulate the antigen-presenting cell to secrete pro-inflammatory cytokines that are required for T-lymphocyte activation (Villadangos et al., 2005).

Highly reactive microglial cells increase the expression of the membrane protein referred to as MHC class $\mathrm{II}$, which allows microglia to readily present antigen to lymphocytes. Given that MHC class II expression is elevated on microglia within the SNc of PD patients (Villadangos et al., 2005), it is possible that microglial-lymphocyte interactions are abnormally enhanced in PD (Teismann et al., 2003). Indeed, MPTP caused infiltration of $\mathrm{CD}^{+}$and $\mathrm{CD}^{+} \mathrm{T}$ lymphocytes, along with enhanced microglial staining and transgenic mice lacking only $\mathrm{CD}^{+}$(not $\mathrm{CD} 8^{+}$) $\mathrm{T}$-lymphocytes were protected from the toxic effects of MPTP (Brochard et al., 2009; Kurkowska-Jastrzebska et al., 1999a). Thus, it was posited that MPTP induced DA cell death may be partially mediated by $\mathrm{CD}^{+} \mathrm{T}$ lymphocytes. This appears to be in sharp contrast to other studies showing that adoptive transfer of co-polymer-1 vaccine protected DA neurons against MPTP by generating non-encephalitic $\mathrm{CD}^{+}$T-lymphocyte infiltration (Benner et al., 2004). Furthermore, this infiltration was associated with suppression of microglia activity and a subsequent increase of GDNF release from astrocytes within the SNc (Benner et al., 2004).

\section{Neuroinflammatory mediated neurodegeneration: Role for microglia}

Presently there is an ongoing debate as to the role microglia and perivascular macrophages play in $\mathrm{PD}$, whether they are primarily responsible for $\mathrm{DA}$ degeneration or a secondary reaction acting to remove the dead/dying neurons. In reality, it is likely that microglia provide some beneficial support to neurons, as well as release toxic by- 
products as a consequence of the respiratory burst. As will be discussed shortly, the various stages of the disease, as well as other factors such as the degree of cellular distress could result in a "waxing and waning" of microglial response.

Microglia and perivascular macrophages are similar: they are both of monocytic origin and primarily act as phagocytes. However, they do differ from each other in many ways i.e. anatomical location, phenotypic expression and functionality. Microglia can be found strictly within the brain parenchyma, functioning as immunomodulatory cells, whereas, perivascular macrophages are generally only found circulating within the cerebrospinal fluid of the perivascular space (Audoy-Remus et al., 2008). During nonpathological conditions microglial cells detect and react to modifications of the local environment (Davalos et al., 2005; Nimmerjahn et al., 2005).

In the case of injury or disease, communication between neurons and microglia, endothelial cells and circulating leukocytes are thought to occur through ATP gradients and the release of heat shock proteins from sick/dying cells (Matzinger, 2002; Popovich and Longbrake, 2008). As neurons succumb to metabolic stress, they release ATP into the extracellular space, inducing the propagation of calcium waves in nearby astrocytes which in turn causes the release of more ATP. Subsequently, microglial cells will recognize these ATP gradients and migrate along these gradients to facilitate the removal of these sick / dying cells (Cotrina et al., 2000; Davalos et al., 2005; Koizumi and Fujishita, 2007). In this regard, microglia perform an essential neuroprotective housekeeping function (Popovich and Longbrake, 2008; Streit et al., 2005). In the case of PD, these 'danger' signals (e.g. ATP or heat-shock proteins) released from injured and dying cells may be subtle and occur over a prolonged period of time (Popovich and 
Longbrake, 2008). Perivascular macrophages are thought to have differing functions, wherein their close proximity with blood vessels and glia limitans place then in an ideal position to regulate angiogenesis, and promote stabilization of blood vessels, phagocytosis and antigen presentation (Harling-Berg et al., 1999).

Post-mortem analysis of PD brains demonstrated profound microgliosis in the SNc and to a lesser extent the striatum (McGeer et al., 1988). This was supported by Langston et al, 1999 who showed that humans exposed to MPTP displayed heightened microglial activation within the SNc 16 years following MPTP exposure (Langston et al., 1999). Initially it was thought that microglia were evenly distributed in the brain; however, Lawson et al 1990 showed that there seems to be a particularly high concentration of microglia in the $\mathrm{SNc}$, olfactory telencephalon, basal ganglia, and hippocampus, which coincidently are regions are most affected by $\mathrm{PD}$ and $\mathrm{AD}$, respectively. In this regard, the high concentration of microglia may explain why these brain regions are preferentially targeted in the aforementioned diseases. The fact that the SNc contains a higher density of microglial cells then the cortex and other brain regions is one explanation as to why the SNc may be especially vulnerable to immunological and environmental toxic insults (Kim et al., 2000).

The reactivity state of microglia varies along a spectrum ranging from resting to hyperactive and is under the strict control of several regulatory proteins. Whether microglia take on a more M1 (a state associated with the respiratory burst and the release of pro-inflammatory cytokines) or M2 (basal state often associated with the release antiinflammatory and neuroprotective factors) phenotype depends on the signals they receive (Michelucci et al., 2009). For the most part, microglia take on a M2 phenotype, acting as 
sentinels slowly proliferating and removing any debris by a means of phagocytosis (Davalos et al., 2005; Kumar and Jack, 2006; Nimmerjahn et al., 2005). In contrast to perivascular macrophages, "resting" microglia (M2) are ramified; meaning thin processes extend from the cell body into the surrounding microenvironment. The immunological phenotype (e.g. surface receptor expression) of this state is characterized by low expression of MHC proteins, CD45, CD14 and CD11b receptors (Aloisi, 2001; Kreutzberg, 1996).

Together with astrocytes, microglia work to maintain a homeostatic microenvironment within the brain, responding to changes in the CNS such as neurotransmitter imbalances as well as infiltrating peripheral immune cells (Bessis et al., 2007). Compelling evidence suggests that microglial cells in a M2 state perform neuroprotective functions in PD, at least in the short term, by secreting trophic factors such as nerve growth factor (NGF), neurotrophin (NT)-3 and brain derived neurotrophic factors (BDNF) (Baquet et al., 2005; Peterson and Nutt, 2008). However, as the disease progresses, cytotoxic substances promoted by DA cell death (e.g. enhanced extracellular debris, oxidative stress and pro-inflammatory cytokine release) cause microglia to undergo changes in their activational state whereby increasing both activation markers and cell adhesion molecules, resulting in a more hyper-reactive state, characteristic of the M1 phenotype (Gao and Hong, 2008). Despite that fact that microglia will undergo functional changes with "normal" aging (heightened activation and reduced proliferative ability) the degree of activation in age-dependent neurodegenerative diseases appear to be substantially different (Conde and Streit, 2006a; Conde and Streit, 2006b). Indeed, in the case of PD, microglial cells can undergo extreme morphological changes and adopt an 
M1 phenotype, wherein their processes retract inward and the cell adopts an amoeboidlike appearance, eventually becoming phagocytic, akin to the perivascular macrophage. Paralleling these morphological changes, these hyper-active microglia enhance the expression of ICAM, MHC class II, complement receptors while at the same time release IL-1 $\beta$, IL-6, IFNs and TNF- $\alpha$ in an attempt to combat infections (Glezer et al., 2007; Hirsch et al., 2005; Przedborski et al., 1996)

An M1 microglial phenotype has been implicated in the progression of toxininduced degeneration of the nigrostriatal pathway (Barcia et al., 2004; McGeer et al., 2003) and are likely responsible for enhancing DA neuronal loss especially following an immunological insult such as LPS. Indeed, priming the SNc with LPS augmented the loss of DA neurons provoked by paraquat and this effect appeared to be dependent upon the manifestation of an M1 phenotypic state of microglial (Mangano and Hayley, 2009). Corroborating these findings, blocking the induction of a M1 microglial state using antiinflammatory drugs such as minocycline, dexamethasone, 3-hydroxmorphinan and NSAIDs protected SNc DA neurons in various animal models of PD (Cassarejos et al., 2006; Du et al., 2001; Kurkowska-Jastrzebska et al., 2004; Quintero et al., 2006; Wu et al., 2002). Thus, in contrast to the beneficial effects M2 microglia, hyper-reactive M1 microglia can produce excessive amounts of inflammatory mediates that can be neurotoxic and lead to neurodegeneration (Block et al., 2007; McGeer et al., 2005)

It is important to mention that, regulatory mechanisms encompassing both antiinflammatory cytokines (IL-4 and IL-10) and inhibitory proteins such as inhibitor $\kappa \mathrm{B} \alpha$ $\left(\mathrm{I}_{\mathrm{k}} \mathrm{B} \alpha\right)$ MAP kinase phosphatase-1 (MKP) and suppressors of cytokine (SOCs) signalling are responsible for suppressing microglial-dependent inflammatory pathways (Glezer et 
al., 2007). Deficiencies in these anti-inflammatory mechanisms can potentially result in aggravated neuronal injury and synaptic dysfunction by altering neurovascular blood flow, BBB permeability, nutrient supply, secretion of matrix molecules, or even by preventing clearance of toxic molecules. Essentially, under non-pathological conditions, the brain is thought to be under constant immunosuppression by IL-10, IL-4 and neurotrophins (e.g. tumor growth factor, and NGF), as well as BDNF (Neumann et al., 1998; Soulet and Rivest, 2008). In fact, IL-4 and IL-10 can promote a M2 microglial phenotype that favours neuronal survival and is associated with increased expression of chemokine ( $\mathrm{C}-\mathrm{C}$ motif) receptor 2 (CCR2) and scavenger receptors, as well as the release of growth factors (Sozzani et al., 1998; Sulahian et al., 2000). Moreover, electrically stimulated neurons (in vitro) have also been reported to suppress MHC expression on microglia through the modulation of the p75 receptor (Neumann et al., 1998). Disturbances of such immunosuppressive processes could lead to chronic inflammation and breakdown of the BBB.

\section{Oxidative stress in PD-pathology: Role for pro-inflammatory cytokines}

Upon contact with sick/dying neurons, innate immune cells such as microglia and macrophages typically release ROS (superoxide and nitric oxide; NO) to initiate the removal of these cells (Gao and Hong, 2008). Provided that these immune cells are only transiently activated, the release of ROS is generally not detrimental to nearby neurons (Gao and Hong, 2008). In addition, several endogenous anti-oxidant systems (e.g. superoxide dismutase, glutathione peroxidase, and catalase) typically neutralize circulating ROS and prevent these free radicals from damaging healthy neurons. However, if microglia are chronically activated, endogenous anti-oxidant systems can become overwhelmed by the accumulation of ROS (Hald and Lotharius, 2005). Indeed, 
post-mortem analyses have revealed a reduction in anti-oxidants (e.g. glutathione) and high levels of lipid peroxidation (indicative of ROS induced oxidative stress) in the SNc of PD patients (Alam et al., 1997; Dexter et al., 1989; Dexter et al., 1994). Furthermore, animal models of the disease have consistently demonstrated that damaged DA neurons are associated with augmented levels of pro-oxidant factors (Anrather et al., 2006; Gao et al., 2002a; Gupta et al., 2010; Mander and Brown, 2005; Pawate et al., 2004; Prasad et al., 2007). Indeed, rodents over expressing superoxide dismutase or glutathione peroxidase are protected against pesticides paraquat and maneb (Thiruchelvam et al., 2005). As such, PD patients may be more susceptible to oxidative stress because they possess basally lower levels of SNc anti-oxidants in comparison to non-PD patients (Bharath et al., 2002).

The primary oxidant producer, NADPH oxidase, is a membrane-bound enzyme of the innate immune system designed to kill microbes, it is highly expressed in granulocytes, macrophages and even microglia (Anrather et al., 2006). NADPH oxidase will catalyze the reduction of molecular oxygen into superoxide by acting as an electron donor. Superoxide has the potential to be dangerous because it is a ROS intermediate, acting as the starting material for generating a variety of highly toxic oxidants including hydrogen peroxide, hydroxyl radicals, singlet oxygen and peroxynitrite (Anrather et al., 2006). Chronically activated microglia can produce large quantities of superoxide via NADPH oxidase in response to variety of toxins including immunological (e.g. LPS) and/or environmental toxins. Over-activation of this enzyme has been postulated to increase the oxidant load to nearby DA neurons (i.e. accumulation of quinolinic acid, ROS, NO) (Mosley et al., 2006). 
Under basal conditions, NADPH oxidase remains inactive, as the core components $\left(\mathrm{p} 40^{\mathrm{PHOX}}, \mathrm{p} 47^{\text {PHOX }}\right.$ and $\mathrm{p} 67^{\mathrm{PHOX}}$ ) reside in the cytosol. In order to activate NADPH oxidase, p47 ${ }^{\text {PHOX }}$ is phosphorylated and stimulates the migration of all three cytosolic subunits to the outer membrane of the microglial cell surface, where they bind with nucleotide-binding proteins Rac1A and Rac2. Collectively, these will activate the catalytic subunit gp91, which is located on the outer surface of the microglia, catalyzing the reaction of oxygen with NADPH oxidase to form ROS (Mosley et al., 2006). Postmortem analysis of $\mathrm{PD}$ brains revealed an up-regulation of gp91 within the SNc, suggesting that NADPH oxidase may be chronically activated in these patients (Gao et al., 2003b; Wu et al., 2003). Similarly, MPTP induced DA neuron death occurred concomitantly with microglial-induced NADPH oxidase production of ROS (Gao et al., 2003b; Wu et al., 2003) and pharmacological inhibition or genetic deletion of NADPH oxidase subunits protected DA neurons from several DA toxins, including paraquat (Purisai et al., 2007).

It is important to mention that the majority of superoxide is produced by NADPH oxidase and can react rapidly with iNOS produced nitric oxide to form the highly dangerous and stable oxidant peroxynitrite. Unlike superoxide, this free radical compound can easily penetrate the outer membrane of DA neurons and cause nitration of tyrosine residues (3-nitrotyrosine) on cellular proteins resulting in structural and functional alterations of nearby neurons (Ischiropoulos and Beckman, 2003). Indeed an up-regulation of iNOS was reported within the SNc of PD patients (Knott et al., 2000) and markers of oxidative stress including 8-hydroxy-2-deoxyguanosine (a marker of DNA damage caused by peroxynitrite) and malondialdehyde (the final marker for lipid 
peroxidation induced by free radicals) are both are elevated that in the plasma of PD patients (Chen et al., 2009). Therefore, it is highly likely that free radicals such as nitric oxide and peroxynitrite contribute to the demise of DA neurons. Moreover, rodent studies using transgenic mice lacking a functional NADPH oxidase enzyme or those deficient in iNOS all displayed significantly less DA neuronal loss following either 6-OHDA, MPTP, rotenone, or paraquat (Cheret et al., 2008; Davalos et al., 2005; Gao et al., 2003a; Gao et al., 2003b; Knott et al., 2000; Purisai et al., 2007; Vila et al., 2001; Wu et al., 2002; Wu et al., 2005)

\section{The role of pro-inflammatory cytokines and their downstream signalling factors in} Parkinson's disease

Cytokines have been implicated in acute and chronic neuronal demise (Anisman et al., 2008). Clinical studies revealed augmented levels of several pro-inflammatory cytokines (TNF- $\alpha$, IL-6, IL-1 $\beta$, IFN- $\gamma$ ) in post-mortem brain as well as in the blood and/or cerebrospinal fluid of patients with stroke, head injury, MS, AD and PD (Basic Kes et al., 2008; Brodacki et al., 2008; Goodman et al., 2008). In particular, a recent study found that PD patients had elevated basal and LPS-induced blood levels of numerous pro-inflammatory cytokines, including MCP-1, RANTES, MIP-1 $\alpha$, IL-8, IFN$\gamma$, IL- $1 \beta$ and TNF- $\alpha$; and significant correlations were observed between cytokine levels and the severity of Parkinsonism (Reale et al., 2009). Although many of these findings have been recapitulated in animal models, it is still uncertain whether these cytokines primarily play a neuroprotective or neurodestructive role. It may be that relatively low endogenous cytokine levels act in a protective capacity to buffer against damage related to death processes, whereas relatively high levels of these factors contribute to neuronal 
damage (Bruce et al., 1996). Indeed, low levels of cytokines can provoke the release of potentially beneficial trophic factors (BDNF, GDNF) and free radical scavengers, but elevated levels can activate oxidative-inflammatory cascades or even induce apoptotic death (Rogers et al., 2001; von Boyen et al., 2006). For instance, mice genetically lacking TNF- $\alpha$ receptors (thereby removing the influence of low endogenous levels of TNF- $\alpha$ ) were more susceptible to ischemic injury (Bruce et al., 1996); yet, administration of exogenous TNF- $\alpha$ at the time of ischemia exacerbated neuronal death (Barone et al., 1997). Likewise, administration of the endogenous IL-1 antagonist, IL-1ra, reduced infarct size in response to middle cerebral artery occlusion and prevented the accumulation of inflammatory infiltrates within the area of damage (Barone et al., 1998), suggesting a prominent destructive role for IL-1 in acute cerebrovascular insults. In effect, the concentration as well as timing of cytokine exposure likely determines whether primarily protective or deleterious consequences arise from these immunotransmitters.

Interleukins and tumor necrosis factor- $\alpha$ in Parkinson's disease

Caspase-1, cleaves the precursor, pro-IL-1, to form the mature and biologically active IL-1 $\alpha$ and IL-1 $\beta$ cytokines (Thornberry et al., 1992). A portion of the synthesized IL-1 is secreted in a soluble form, but a portion is retained within the cell membrane (Dinarello, 1988). Both the soluble and membrane-bound forms of IL-1 are biologically active, particularly with respect to lymphocyte activation (Dinarello, 1988). Although IL1 is a potent pro-inflammatory agent, it also has many other actions, including appetite regulation, insulin secretion and neuronal phenotype development (Plata-Salaman, 1994). IL-1 signalling is dependent upon its type I receptor and the IL-1 receptor accessory protein, which are located on adjacent portions of the membrane (Rothwell and Hopkins, 1995). 
Based upon its involvement in the wasting syndrome (cachexia) often seen in cancer patients, the pro-inflammatory cytokine TNF- $\alpha$ was previously referred to as cachectin. Interestingly, this cytokine was at one point considered to be a promising treatment for some types of cancer (e.g., melanoma) (Bennani-Baiti and Davis, 2008). Much like IL-1 $\beta$, TNF- $\alpha$ is a pleiotropic cytokine, which exerts a wide array of actions on numerous cell types. For instance, it has physiological actions on bone osteoclasts (important for rheumatoid arthritis), mononuclear and polymorphonuclear blood cells, fibroblasts, skin keratinocytes, insulin sensitive adipocytes, as well as brain neurons and glial cells (Eigler et al., 1997). Like other cytokines, TNF- $\alpha$ typically acts locally at the site of generation; however, small amounts of the cytokine are found circulating in the bloodstream.

Mounting evidence suggests a role for ILs and TNF- $\alpha$ in PD. Specifically, postmortem analyses of PD brain tissue revealed increased expression of TNF- $\alpha$ and its related Fas receptor, as well as the cytokines IFN- $\gamma$, IL-1 $\beta$ and IL-6 (Nagatsu et al., 2000). Likewise, in animals, MPTP induced alterations of pro-inflammatory cytokine genes, including those encoding IL-1 $\beta$ and TNF- $\alpha$ (Bian et al., 2009; Mandel et al., 2000b); similarly, 6-OHDA increased levels of these cytokines within the SNc and striatum (Jin et al., 2008). Indeed, an increasing number of studies are beginning to assess the impact of cytokine manipulations on PD-like pathology. In this regard, both systemic and central administration of IL-1 $\beta$ was reported to affect SNc DA neuronal survival. Indeed, pharmacological inhibition of IL- $1 \beta$ attenuated the loss of DA neurons provoked by intra-SNc infusion of LPS together with 6-OHDA injection (Koprich et al., 2008). Moreover, direct application of IL-1 $\beta$ augmented the neurodestructive effects of 6- 
OHDA upon cultured midbrain neurons (Pott Godoy et al., 2008). However, somewhat surprisingly chronic adenoviral induced expression of IL-1 $\beta$ in the striatum also induced a loss of SNc DA neurons (Pott Godoy et al., 2010), suggesting that the cytokine can exert damaging effects upon DA terminals that results in the retrograde destruction of upstream soma. Importantly, the IL-1 $\beta$ induced loss of neurons was associated with motor impairment and an enhanced microglial response; and anti-inflammatory treatment prevented these effects (Pott Godoy et al., 2010). Yet, older studies reported that central infusion of IL-1 $\beta$ protected DA neurons from 6-OHDA and MPTP toxicity and induced dendritic branching from residual neurons following SNc lesion (Saura et al., 2003; Wang et al., 1994). The discrepancies between the studies remain to be explained but likely stem from dose and timing considerations, since, as already mentioned, some cytokines might have both protective and deleterious effects depending on their concentration and the state of the microenvironment in which they act.

Involvement of TNF- $\alpha$ in PD, like IL-1 $\beta$, is somewhat controversial, with two conflicting reports indicating that TNF- $\alpha$ deletion either protected striatal terminals and normalized DA levels in MPTP-treated mice (Ferger et al., 2004; Sriram et al., 2002) or increased DA metabolism, without necessarily affecting neuronal survival (Rousselet et al., 2002). Interestingly, in one study there was no effect of intra-SNc infusion of TNF- $\alpha$ or IL-1 $\beta$ either alone or together upon neuronal survival (Castano et al., 2002), but the source for this outcome is uncertain. More recently, adenoviral vector mediated longterm expression of TNF- $\alpha$ within the SNc was reported to provoke a progressive loss of DA neurons over 28 days that was associated with irreversible akinesia (De Lella Ezcurra et al., 2010). Likewise, over-expression of a dominant negative TNF- $\alpha$ protein (inhibits 
endogenous TNF- $\alpha$ ) in the SNc ameliorated the loss of DA neurons and motor impairment induced by 6-OHDA treatment (McCoy and Tansey, 2008).

The cytokines IL-1 $\beta$ and TNF- $\alpha$ typically influence central processes through $\mathrm{NF} \mathrm{KB}$, a transcription factor that plays a critical role in the regulation of innate and adaptive immune reactions, including the mobilization of inflammatory chemokines and lymphocyte proliferative responses following infection or traumatic injury (Lucas et al., 2004; Poynter et al., 2004). Indeed, NFkB signalling occurs ubiquitously throughout the brain, and IL-1 $\beta$ infusion into the lateral ventricles induced the translocation of NFKB to the nucleus at several brain regions distal to the site of infusion, including the choroid plexus, ependymal cells, cerebral vasculature and meninges, as well as at the basolateral amygdala (Konsman et al., 2000).

$\mathrm{NF} \kappa \mathrm{B}$ is composed of five subunits, together with a nuclear localization signal, which are normally held in an inactive state by an endogenous inhibitory factor, IאB. However, exposure to inflammatory stimuli triggers the phosphorylation and consequent degradation of $\mathrm{I} \kappa \mathrm{B}$, resulting in the translocation of $\mathrm{NF} \kappa \mathrm{B}$ to the nucleus where it promotes gene expression (Lucas et al., 2004). Immunological insults may initiate this NFkB cascade through the provocation of cytokines, particularly IL-1 $\beta$ and TNF- $\alpha$, which, after binding to their cell surface receptors, stimulate kinases that target IKB for ubiquitination and subsequent proteasomal degradation (Lucas et al., 2004). As well, these cytokines may also affect CNS processes by stimulating NFKB signalling cascades.

To this end, NFאB appears to have potent effects upon CNS processes important for neuronal survival and plasticity. In terms of functional outputs, NFKB is readily 
activated in response to excitatory neurotransmission and is believed to play an important role in learning and memory (Mattson, 2005). The transcription factor may also have a neuroprotective role through the induction of anti-apoptotic proteins, such as $\mathrm{Bcl}-2$ and the antioxidant enzyme, manganese superoxide dismutase (Mattson, 2005). Yet, NFkB signalling may also result in the synthesis or up-regulation of inflammatory cytokines and enzymes, ROS, and excitotoxins that can contribute to neurodegeneration (Aoki et al., 2009; Pahan et al., 2001). For instance, iNOS expression within microglia and astrocytes is readily provoked by $\mathrm{NF \kappa B}$ activation following exposure to cytokines, such as IL-1 $\beta$ or IL-12 (Hartlage-Rubsamen et al., 1999; Pahan et al., 2001). Similarly, stressor exposure may contribute to neurological pathology by affecting NFkB-mediated production of oxidative radicals given that restraint stress was shown to promote neuronal excitotoxicity in rats that was associated with enhanced TNF- $\alpha$ release and NFKB mediated activation of iNOS and COX-2 (Madrigal et al., 2006). Ultimately, a host of factors, including the chronicity and type of inducing stimulus, likely influence whether NFkB activation has protective or detrimental effects upon neuronal survival or functioning.

Emerging evidence for a role of IFN- $\gamma$ signalling in Parkinson's disease

The T-helper type 1 (Th1) pleiotropic pro-inflammatory cytokine, IFN- $\gamma$, has also been implicated as an important player in PD-like pathology. In fact, our own work and that of others revealed that PD patients incurred significantly higher levels of IFN- $\gamma$ in serum and the nigrostriatal pathway when compared to aged matched controls (Gribova et al., 2003; Mount et al., 2007). Paralleling these human studies, genetic ablation of IFN$\gamma$ attenuated the neurodegenerative effects of MPTP on the nigrostriatal system in mice and blunted the neuroinflammatory response engendered by the toxin (Mount et al., 
2007). Similarly, in vitro co-cultures consisting of midbrain neuronal + microglia were protected from rotenone's toxicity when microglial cells were taken from mice deficient for the receptor for IFN- $\gamma$, suggesting that the cytokine is likely originating from and acting through microglia (Mount et al., 2007).

Although IFN- $\gamma$ is virtually undetectable in the healthy brain, central levels of the cytokine are apparent following acute traumatic injury, as well as in association with autoimmune conditions, such as MS (Mana et al., 2006). Indeed, it is well established that IFN- $\gamma$ is produced in the periphery by lymphoid cells (mainly $\mathrm{CD} 4^{+}$lymphocytes and natural killer cells) and these cells can infiltrate the nigrostriatal system following sufficient challenge (Mount et al., 2007). Moreover, emerging evidence indicates that IFN- $\gamma$ may also be produced by resident brain microglia, albeit in low concentrations (Kawanokuchi et al., 2006; Mount et al., 2007). In fact, a recent study reported IFN- $\gamma$ in the supernatants of immune challenged microglia, even in the absence of $T$ cells (Wang and Suzuki, 2007).

Neurotoxicity caused by IFN- $\gamma$ may be directly attributed to its ability to promote transcription of several pro-oxidative factors such as iNOS and gp91[rate-limiting catalyst required for nicotinamide adenine dinucleotide phosphate-oxidase (NADPH) oxidase activation] (Kakar et al., 2005). As well, IFN- $\gamma$ if co-administered with LPS can also enhance the production of several pro-inflammatory cytokines from microglia including IL-6 and TNF- $\alpha$ (Lafortune et al., 1996). This is particularly important given that chronic activation of TNF- $\alpha$ can exaggerate a neuroinflammatory responses and enhance DA neuronal loss via p55 TNF-R1 mediated apoptosis (Hayley et al., 2004a). 
Binding of IFN- $\gamma$ to its receptor (IFN- $\gamma-\mathrm{R}$ ) on microglia can induce a series of events that are mediated by JAK/STAT. Essentially IFN- $\gamma-\mathrm{R}$ will remain inactive until IFN- $\gamma$ catalyzes the activation of the enzymes JAK1 and JAK2, which are linked to the cytoplasmic tail of the receptor. Thereafter, two STAT molecules are recruited and bind to the paired docking sites on the activated receptor complex located on the JAKs (Thomson and Lotze, 2003). During this activation process, the STAT molecules are phosphorylated and dissociate from the IFN- $\gamma-\mathrm{R}$ in order to form homo- or hetero-dimers. The activated STAT1 homodimers or STAT1-STAT2 heterodimer will translocate to the nucleus and promote the transcription of several genes including MAP kinases, CREB, and iNOS (Planas et al., 2006; Thomson and Lotze, 2003).

Prolonged activation of JAK-STAT signalling following an immune challenge is likely caused by concerted actions of several cytokines besides IFN- $\gamma$, including IL-6 and IL-10 (Arimoto et al., 2006; Bolin et al., 2002; Bolin et al., 2005; Goodwill et al., 2007; Qian et al., 2006). Indeed, these cytokines promote a wide range of effects mediated through JAK/STAT, wherein their concentration and timing of exposure appear to dictate the type of response. Finally, it is important to mention that potential cross-talk between JAK-STATs and any other related pathways that might be coincidentally activated. Indeed, besides the typical aforementioned cytokines that induce JAK-STAT signalling, several other cytokines, such as IL- $1 \beta$ and TNF- $\alpha$, that act through alternative pathways (e.g. NFאB, MAP kinases) following a variety of immune challenges. Ultimately, the neuronal outcomes provoked will depend upon the overall "sum" of each of these seemingly disparate pathways. 


\section{Therapeutics of Parkinson's disease: past, current and future}

Recent neuroimaging studies have suggested that the preclinical stage for PD is approximately 4-5 years prior to symptom onset and the rate of DA cell loss is roughly $10 \%$ each year during the early stages of the disease (Dunnett and Bjorklund, 1999). Based on the multiple aforementioned mechanisms implicated in nigrostriatal degeneration (e.g. accumulation of oxidative factors, chronic inflammation, apoptosis and mitochondrial dysfunction), different stages of the "neurodegenerative cycle" may offer potential for diverse neuroprotective strategies. In this regard, neurotrophic, antiinflammatory and anti-apoptotic factors may offer beneficial effects at different stages of disease progression.

The most commonly prescribed treatment option for PD is L-DOPA (oral administration of the precursor for DA) because it can easily cross the BBB and immediately compensate for DA loss. Despite the fact that ${ }_{L}$-DOPA is effective at alleviating akinesia (inability to initiate movement) and rigidity during the early and midstages of the disease (Dunnett and Bjorklund, 1999), a tolerance to ${ }_{\text {L-DOPA develops }}$ during the late stages of PD. As well as a series of unwanted side-effects, such as dyskinesia (controlled involuntary motor gestures) also eventually emerge with this treatment. Chronic fluctuations of DA levels associated with ${ }_{L}$-DOPA treatment itself might actually accelerate neurodegeneration, owing to the high rate of oxidative metabolites generated with excessive DA concentrations (Marsden and Parkes, 1977). Hence, patients are often forced to reduce or halt treatment during the later stages of the disease (Marsden and Parkes, 1977).

Newer drug treatment options have recently been explored, which are not accompanied by such debilitating side effects. For instance, DA agonists, inhibitors of 
catechol-O-methyltransferase and monoamine oxidase $\mathrm{B}$, as well as antioxidants and DAT blockers have recently been employed (Dunnett and Bjorklund, 1999). Unfortunately, like ${ }_{L}$-DOPA, the efficacy of these treatments also decline with disease progression. More recently, neurosurgical approaches such as thalamotomy and deep brain stimulation have been shown to successfully reduce the core symptoms caused by PD such as tremors. Like most therapeutic treatments of PD, surgical procedures do not halt disease progression; they merely alleviate the core motor symptoms (Bronstein et al.; Marsden et al., 1997). The clinical benefit from such surgical interventions is also relatively short lived (lasting 4-5 years) (Rodriguez-Oroz et al., 2005).

Several anti-oxidative treatment options or adjunct therapies have been explored for PD, including ongoing clinical trials involving vitamin $\mathrm{E}$ and deprenyl. Other drugs, such as glutathione-enhancing agents and ion chelators are also presently being explored in animal models to determine their neuroprotective effects against DA toxins (Dunnett and Bjorklund, 1999). Besides pharmacological approaches, new genetic technologies, particularly siRNA, may also provide the opportunity to selectively manipulate different pro-death mechanisms in PD.

One exciting new avenue for treating PD involves the use of trophic factors in an attempt to stabilize neuronal viability and even promote some degree of recovery following long standing disease. Indeed, recent studies have revealed that there is a reduction of the trophic cytokine, BDNF within the SNc of PD patients (Mogi et al., 1999; Salehi and Mashayekhi, 2009). Given those receptors for BDNF have been found on SNc DA neurons (Murer et al., 2001), a reduction in trophic support to these neurons may increase their vulnerability to later toxin exposures. In addition to providing trophic 
support to DA neurons, it is hypothesized that BDNF also dampens inflammatory and oxidative pathways through the activation of its receptor, tyrosine kinase receptor $\mathrm{B}$ (Murer et al., 2001). For example, BDNF can promote the survival and differentiation of mesencephalic DA neurons as well as protect against MPTP and 6-OHDA insults in vitro (Murer et al., 2001). Other studies show that intrastriatal injection of BDNF prevents 6OHDA induced loss of SNc DA neurons and normalizes apomorphine-induced rotation, which is a measure of asymmetrical DA functioning resulting from unilateral DA loss (Shults et al., 1995). Therefore, numerous studies support the use of neurotrophic factors, such as BDNF as a treatment option for PD patients (Peterson and Nutt, 2008).

Another neurotrophic factor, glial derived neurotrophic factor (GDNF) belongs to the TGF- $\beta$ superfamily and has emerged as a potential candidate for neuroprotection in PD patients. GDNF is produced primarily by astrocytes, although microglial cells do contribute low levels of GDNF. Despite that fact that post-mortem studies have indicated that GDNF is unchanged in the PD brain (Mogi et al., 2001), the appeal to using GDNF as a therapeutic agent is based on recent studies reporting that the factor has both neuroprotective and neurorestorative properties. In fact, central infusion of GDNF (either intra-SNc or intra-striatal) has been shown to be neuroprotective against virtually all animal models of PD (Ai et al., 2003; Fox et al., 2001; Gash et al., 1998; Gash et al., 1996; Kearns et al., 1997; Tomac et al., 1995). In particular, a central infusion of GDNF not only attenuates the destructive effects of MPTP by restoring DA levels and fibre density, but also promotes motor recovery (Tomac et al., 1995). Importantly, GDNFinduced restoration of DA functioning persists months after trophic support was terminated (Grondin et al., 2002; Kirik et al., 2004; Maswood et al., 2002). 
Due to the success of GDNF in rodent and non-human primate models of $P D$, the growth factor has been used in several clinical trials. Indeed, direct infusion of GDNF into the putamen induced significant improvements in motor symptoms as early as three months after commencing the treatment and was associated with minimal side effects (Gill et al., 2003; Patel et al., 2005; Slevin et al., 2005). However, upon closer investigation, the improvements in dopamine storage were restricted to the immediate area surrounding the catheter used during the growth factor infusion (Gill et al., 2003; Love et al., 2005). These latest findings are consistent with findings from non-human primates that reported similar clinical efficacy, coupled with axonal sprouting, following GDNF infusion into the basal ganglia (Gill et al., 2003). However, a randomized placebocontrolled study was unsuccessful at replicating these beneficial effects (Lang et al., 2006). In addition, a few patients developed antibodies against the infused GDNF peptide, which may interfere with the therapeutic benefits and others appeared to be more vulnerable to opportunistic infections (Gill et al., 2003; Lang et al., 2006). The discrepancies in the data may be due, in part to variations in the catheter design and positioning, as well as drug dosage.

Given the inconclusive beneficial effects coupled with the side-effects associated with GDNF delivery, several new techniques are presently being developed to get around the fact that GDNF do not cross the BBB. These include newer generation viral vectors that temporarily disrupt the BBB to allow central infiltration (Doolittle et al., 2000), implantation of trophic factor secreting neural progenitor cells (Behrstock et al., 2006), encapsulating trophic factors to facilitate BBB penetration (Juillerat-Jeanneret and 
Schmitt, 2007; Pardridge, 2002), as well as breaking trophic factor into small bioactive fragments to again enhance their infiltration (Peleshok and Saragovi, 2006).

Alternatively, finding novel neurotrophic factors that can cross the BBB would be desirable. In this regard, granulocyte-macrophage colony stimulating factor (GM-CSF) is a hematopoietic cytokine with well documented trophic actions in the periphery that has been shown to infiltrate the CNS and accumulate in reasonable levels within the brain (Franzen et al., 2004; McLay et al., 1997; Thomson and Lotze, 2003). Its basic function is to promote the differentiation and maturation of peripheral innate immune cells, including neutrophils and dendritic cells. Indeed, GM-CSF is routinely administered to cancer patients to reduce febrile neutropenia (fever response) by accelerating formation of neutrophils from granulocyte progenitors (Dale, 2002).

GM-CSF receptor is a heterodimer consisting of a ligand-binding epitope and a signal transduction subunit that interestingly enough, in humans is shared with the IL-3 and IL-5 receptors (Whetton and Dexter, 1989). GM-CSF is mainly produced by peripheral immune cells ( $\mathrm{T}$ lymphocytes, macrophages, and NK cells) and to a less degree central astrocytes and microglia (Malipiero et al., 1990; Thomson and Lotze, 2003). As the name suggests, this cytokine acts to rapidly mobilize and stimulate the proliferation and maturation of myeloid progenitors, precursors of neutrophils, monocytes, macrophages, and eosinophils to contend with infectious or traumatic insults (Reddy et al., 2009). Recently GM-CSF receptors were discovered on neural progenitor cells, suggesting that GM-CSF may also stimulates the maturation of neurons (Ha et al., 2005; Kim et al., 2004). Such an effect may be one of many mechanisms through which GM-CSF can improve neurological performance following acute CNS injuries such as 
spinal cord injury and cerebral ischemia (Kawada et al., 2006; Nakagawa et al., 2006; Yoon et al., 2007). However, the therapeutic effect of this trophic cytokine in neurodegenerative diseases is unclear.

In addition to its pro-inflammatory effects, GM-CSF has recently been shown to have potent growth factor-like properties within the CNS. In fact, systemic exposure to GM-CSF induced spontaneous axonal regeneration and promoted functional recovery from traumatic spinal cord injury (Bouhy et al., 2006; Ha et al., 2005; Huang et al., 2007) and reduced infarct volume and improved neurological function following ischemia (Nakagawa et al., 2006; Schabitz et al., 2008). Moreover, GM-CSF activation of microglia/macrophages can result in vascular proliferation and increase collateral blood flow in patients suffering from coronary artery disease (Nakagawa et al., 2006).

Paralleling these findings, GM-CSF has also been shown to protect CNS from injury by counteracting apoptotic cell death. Specifically, GM-CSF has anti-apoptotic effects, as indicated by its ability to inhibit the pro-apoptotic proteins, p53 and Bax, while increasing levels of the anti-apoptotic factor, $\mathrm{Bcl}-2$; subsequently inducing the proliferation and differentiation of neural stem cells following spinal cord injury (Huang et al., 2007). Similarly, GM-CSF attenuated the neurodegenerative effects of MPTP both in vivo and in vitro by promoting the expression of $\mathrm{Bcl}-2$ and down-regulating the expression of Bax (Kim et al., 2009a). The neuroprotective properties associated with this trophic cytokine are beginning to gain momentum and in the future GM-CSF may become a potentially useful treatment option for PD. 


\section{Research Objectives}

PD has emerged as a multifaceted disease, wherein intermittent exposures to various environmental (e.g. pesticides, heavy metals, infections) and immunological toxins are proposed to take a toll on the CNS. That said, it is likely that some genetic predisposition might play a role in at least a subset of PD cases. It is our contention that although relatively low levels of environmental toxins might not have obvious immediate consequences, collectively these agents set in place a series of inflammatory changes that eventually culminates in CNS pathology. Specifically, accumulation of free radicals and other factors caused by prolonged pesticide exposure will eventually overwhelm the nigrostriatal system and give rise to a chronic pro-inflammatory state, as indicated by an up-regulation of pro-inflammatory cytokines and pro-oxidant mediators. Given the complex aetiology of PD and substantial evidence implicating multiple converging prodeath pathways (pro-apoptotic, pro-oxidative, and neuroinflammatory) in the disease, it is not unreasonable to suggest that a combination of drug therapies may be clinically efficacious in preventing ongoing neurodegeneration. The proposed studies of this dissertation characterized the involvement of microglia and the pro-inflammatory cytokine, IFN- $\gamma$, in contributing to the neurodegenerative effects of LPS and paraquat. As well, we also assessed the neuroprotective consequences of the trophic cytokine GMCSF. The general discussion section following the specific chapters puts our findings in perspective and seeks to integrate in an overall model how the inflammatory and trophic cytokines might come to influence neuronal survival. 


\section{The aims of this dissertation sought to assess}

1. The temporal effects of paraquat exposure on the nigrostriatal system.

2. The role of IFN- $\gamma$ in paraquat-inducted toxicity and elucidate the involvement of IFN- $\gamma$ induced microglial-dependent processes in the demise of DA neurons

3. Whether central administration of LPS can sensitize or de-sensitize SNc DA neurons to the effects of paraquat.

4. Whether the trophic cytokine, GM-CSF, can protect DA neurons from the effect of LPS and paraquat.

5. The effectiveness of GM-CSF administration when administered either centrally or systemically. 


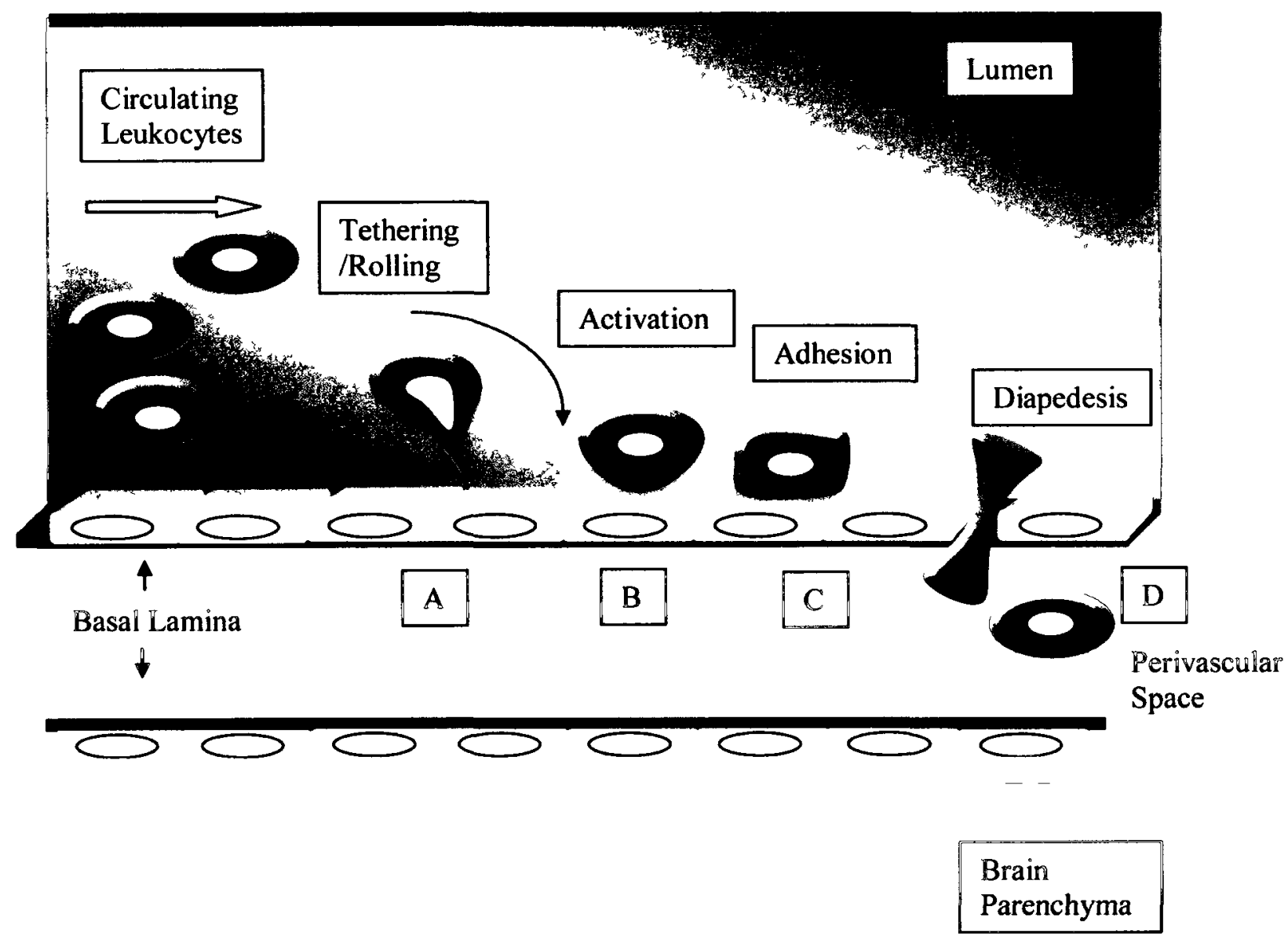

Figure 1.1 Extravasation. The process of leukocyte extravasation across vascular walls and into tissue, including the brain requires interaction between several adhesion and signalling molecules on both the circulating leukocytes and the vascular wall. Circulating lymphocytes regularly make transient contact with the vascular epithelium, a process referred to as tethering. Up-regulation of a selectins on the leukocytes will allow the leukocytes to reduce their velocity and roll along the endothelium surface (A). The leukocytes search for chemotactic factors present on the endothelium (B), which in turn increase the avidity by allow the integrins (i.e.VLA-4 and LFA1) present on the leukocytes to bind to adhesion molecules (C). This enhanced avidity will allow the leukocytes to adhere to the vascular endothelial wall and promoting diapedesis of leukocytes (D). 


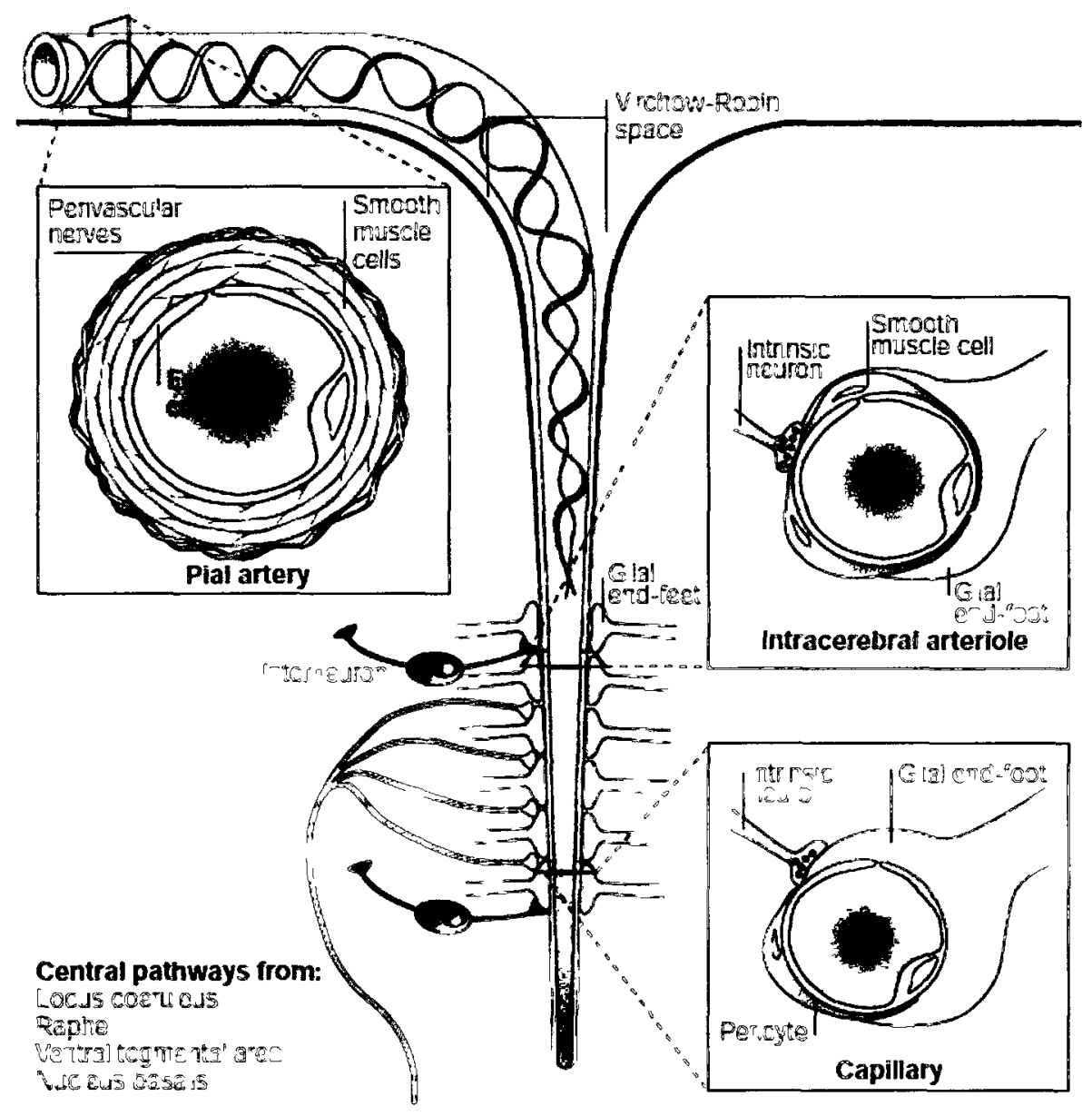

Iadecola, C. (2004) Nature 5:349

Figure 1.2 Anatomy of the Neurovascular Unit. The brain is divided into three layers, the dura (outer), the arachnoid (middle) and the pial (inner) each layer is richly supplied with blood vessels and in close proximity to neuronal processes. The cerebral arteries branch into smaller pial arteries, they run along the surface of the brain. As these arteries penetrate into the brain they are separated from the brain parenchyma by the perivascular space, which is filled with cerebrospinal fluid. As the arterioles penetrate deeper into the brain, perivascular space becomes smaller and eventually the perivascular space disappears. The vascular basement membrane of the arterioles comes in contact with the astrocytic end-feet, forming the capillary. The Intracerebral arterioles and capillaries are also closely associated with neural processes that originate within the CNS. Collectively, the perivascular neurons, astrocytes, and vascular cells constitute a functional unit referred to as the neurovascular unit. The main purpose of the unit is to maintain the homeostasis of the cerebral microenvironment. The neurovascular unit also acts as a defence against deleterious effects of brain injuries. 


\section{Proinflammatory cytokines and Parkinson's disease, 50}

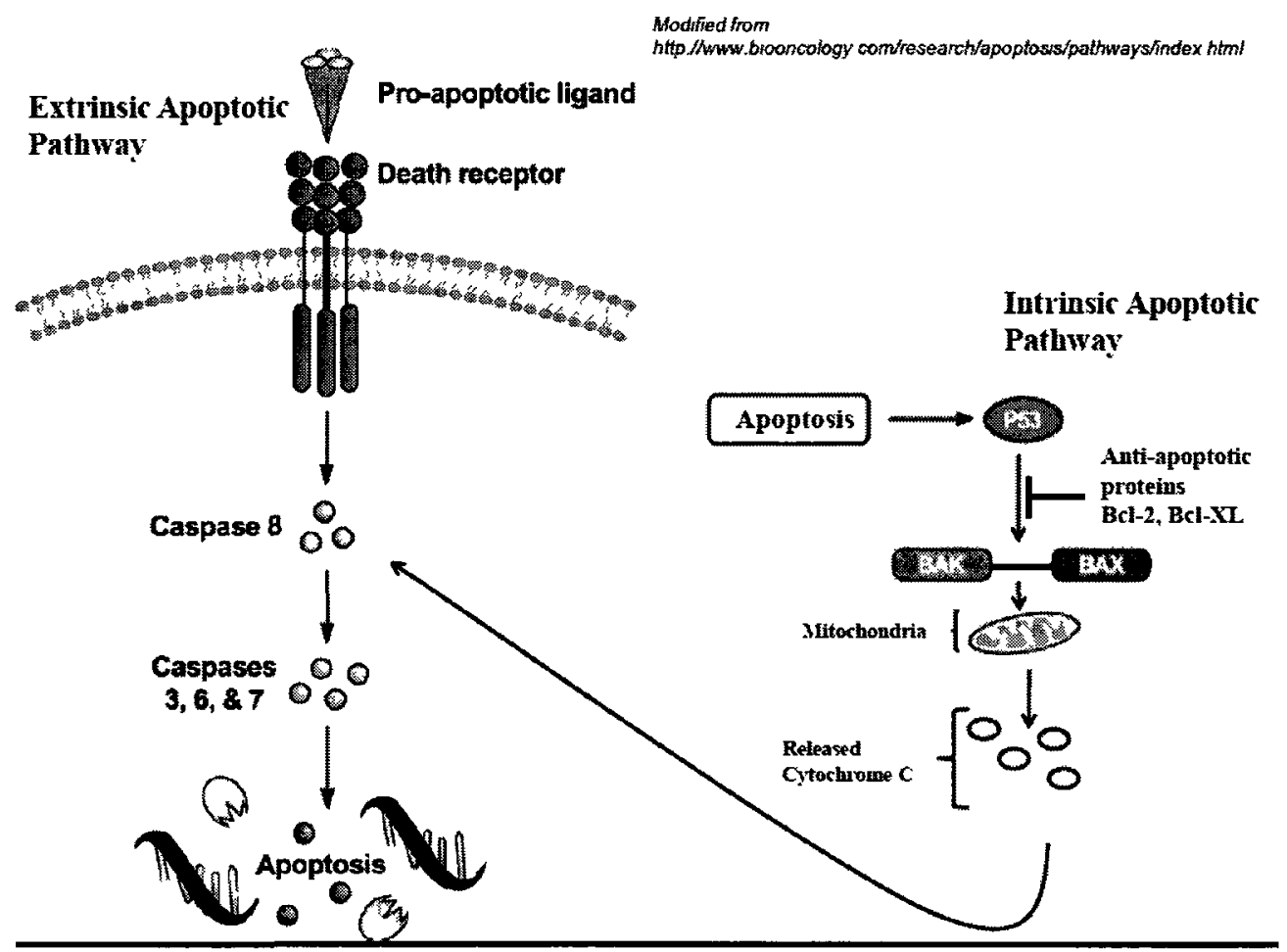

\section{Box 1.1 Apoptotic cell death in PD-like pathology.}

There are two different ways to achieve apoptosis; using the extrinsic (top left) or intrinsic (bottom right) pathway. Both pathways converge on effector caspases and eventually destroy the cell by cutting cellular and nuclear substrates. In the extrinsic pathway utilizes a trimerization of death receptors activated by death ligands. Subsequently, this will to the activation of proteolytic regulatory caspases, such as caspases-8, and eventually caspases-3. The intrinsic pathway involves activation of pro-apoptotic proteins (Bax or Bak) by $\mathrm{p} 53$, which will cause the formation of large mitochondrial pores and the subsequent release of cytochrome $\mathrm{c}$. This in turn activates regulatory caspases, usually involving caspases- 9 , and eventually caspases -3 .

Unlike necrosis which involves swelling and cell lysis, which has profound affects upon neighbouring cells, apoptosis is an active process of programmed death that does not markedly affect the extracellular space (Vila and Przedborski, 2003). The crucial mediators of apoptosis are proteolytic enzymes referred to as caspases, which cleave their substrates after becoming activated in response to intracellular signalling pathways. Upon activation, initiator caspases (caspases 2,8,9, and 10) cleave effector caspases (caspases 3,6, and 7), which are ultimately responsible for mitochondrial damage, nuclear membrane breakdown, DNA fragmentation and inevitably cell death (Vila and Przedborski, 2003). Typically, the cell begins to shrink due to a breaking down of the chromatin in the nucleus (nuclear condensation). Although these cells do not readily spill their contents into the extracellular space, local microglia are mobilized in order to engulf and remove these compromised cells.

In some cases, extrinsic signals such as the pro-inflammatory cytokines, Fas and TNF- $\alpha$, bind to their cell surface receptors (which are located on DA neurons) and promote apoptotic cell death. Essentially, activation of these surface receptors is associated with particular adaptor proteins which contain 'death effector domains' that recruit the initiator caspases-8. Therefore, apoptosis may come about either from exposure to exogenous pro-apoptotic insults or following intrinsic signals that are produced following cellular stress, such as growth factor deprivation.

Enhancing mitochondrial permeability appears to be a common event in all forms of apoptotic cell death. The pro-apoptotic factors Bax, Bad and Bak can promote mitochondrial leakage by increasing outer membrane permeabilization. The formation of these large pores in the mitochondrial outer and inner membranes will cause leakage of mitochondrial toxic factors into the cytosol (Gomez-Lazaro et al., 2008). Essentially, Bax and Bad will translocate to the mitochondria, promoting the release of cytochrome $C$ into the cytosol (via these large pores in the mitochondrial membrane). Cytoplasmic cytochrome $\mathrm{C}$ coupled with then interacts with two cytosolic proteins; apoptotic protease-activating factor and caspase- 9 , which leads to activation of caspase- 3 and ultimately, apoptosis 
Proinflammatory cytokines and Parkinson's disease, 51

Chapter 2

Interferon- $\gamma$ plays a crucial role in paraquat-induced neurodegeneration involving oxidative and pro-inflammatory pathways. 


\section{Abstract}

Exposure to environmental contaminants, particularly pesticides, may be an important etiological factor in Parkinson's disease (PD) and evidence suggests a role for microglia dependent inflammatory and oxidative processes in nigrostriatal pathology induced by such toxins. Yet, the events mediating microglial activation and their effects are not fully known. To this end, we hypothesized that the pro-inflammatory cytokine, interferon-gamma (IFN- $\gamma$ ), may be a prime factor in the pathogenesis of PD, given its critical role in regulating microglial responses to pathogens. Indeed, the present investigation demonstrated that genetic deletion of IFN- $\gamma$ protected substantia nigra pars compacta (SNc) dopamine (DA) neurons from the toxic effects of the pesticide, paraquat, and normalized changes in inflammatory and oxidative factors within this brain region. Specifically, IFN- $\gamma$ knockout prevented the paraquat-induced morphological signs of microglial activation and expression of key NADPH oxidase subunits, while also preventing time-dependent changes in pro-inflammatory enzymes (iNOS, COX-2), cytokines (IL-1 $\beta$, TNF- $\alpha$ ), and signalling factors (JNK, p38, STAT1, NFkB). Moreover, paraquat transiently suppressed SNc expression of trophic and pro-neuroplastic factors (CREB, BDNF), and IFN- $\gamma$ deficiency again reversed these effects. These data suggest that IFN- $\gamma$ is required for paraquat-induced microglial activation and the subsequent elaboration of oxidative and inflammatory cascades underlying $\mathrm{SNc}$ dopaminergic cell loss, as well as the early pesticide induced reductions in trophic support that may render neurons even more susceptible to toxin damage. Targeting IFN- $\gamma$ could thus have therapeutic implications for PD and other neurodegenerative conditions that involve multiple inflammatory pathways. 


\section{Introduction}

The neuropathological processes underlying Parkinson's disease (PD) are characterized by, among other things, a progressive degeneration of dopamine (DA) neurons of the substantia nigra pars compacta ( $\mathrm{SNc}$ ) and consequently a loss of DA release in the striatum (Langston, 2006). Mounting evidence suggests that environmental toxins contribute to PD, with numerous epidemiological studies implicating exposure to heavy metals, infectious agents, and especially pesticides such as paraquat (Liou et al., 1997; Peng et al., 2007; Dhillon et al., 2008; Elbaz et al., 2009). Administration of paraquat (and other pesticides, e.g., rotenone) provoked a loss of SNc DA neurons while inducing PD-like behavioral disturbances, coupled with oxidative and inflammatory stress in nigrostriatal and limbic brain regions (Gao et al., 2003a; Sindhu et al., 2005; Litteljohn et al., 2008; Somayajulu-Nitu et al., 2009; Chen et al., 2010). Beyond their individual consequences, environmental toxins can further enhance neuronal death when exposure occurs in the context of genetic vulnerability (Yang et al., 2007), or following a pre-existing neuroinflammatory response, (Purisai et al., 2007; Mangano and Hayley, 2009).

Microglial-driven neuroinflammation and oxidative stressors appear to be particularly important in the progression of PD (McGeer et al., 1988, 2008; L' Episcopo et al., 2010). Indeed, activated microglia along with up-regulated expression of the enzymes, nicotinamide adenine dinucleotide phosphate (NADPH) oxidase, inducible nitric oxide synthase (iNOS) and cyclooxygenase-2 (COX-2), have been observed within the SNc of post-mortem PD brain, as well as in rodents treated with DA toxins (Hunot et al., 1996; Liberatore et al., 1999; Knott et al., 2000; Gao et al., 2003a; Wu et al., 2003). Correspondingly, genetic or pharmacological inhibition of these inducible enzymes 
conferred some degree of neuroprotection in rodents (Teismann and Ferger, 2001; Feng et al., 2003; Wu et al., 2003; Vijitruth et al., 2006; Qian et al., 2007). The fact that blocking microglial activity using anti-inflammatories, such as minocycline, dexamethasone or 3-hydroxymorphinan, attenuated the neurodegenerative impact of DA toxins (Du et al., 2001; Wu et al., 2002; Kurkowska-Jastrzebska et al., 2004; Zhang et al., 2006), along with findings showing that microglial activation precedes neuronal loss (Kim et al., 2000; Purisai et al., 2007; Huang et al., 2008), supports the notion that microglia are primary pathological contributors to SNc neuronal loss.

The pro-inflammatory cytokine, interferon- $\gamma($ IFN- $\gamma)$, is a critical regulator of microglia, inducing the expression of both oxidative and inflammatory enzymes (NADPH oxidase; iNOS, COX-2) (Eklund and Kakar, 1999; Delgado, 2003; Pawate et al., 2004; Kawanokuchi et al., 2006) and promoting pro-inflammatory cytokine release, many of which [e.g., tumor necrosis factor- $\alpha$ (TNF- $\alpha$ ), interleukin-1 $\beta$ (IL-1 $\beta)$ ] have been implicated in PD (Mogi et al., 1994; Gribova et al., 2003; McCoy et al., 2006, 2007; Koprich et al., 2008). Likewise, we found that IFN- $\gamma$-deficient mice were resistant to the impact of MPTP (Mount et al., 2007) and displayed attenuated neurochemical and behavioral responses to paraquat (Litteljohn et al., 2009).

The mechanisms through which IFN- $\gamma$ might influence DA neuronal survival are not well established, nor has its role been evaluated using an ecologically relevant toxin model of PD. To this end, we hypothesized that IFN- $\gamma$ deficiency would prevent SNc DA neuron loss following administration of the pesticide, paraquat, and that such an effect would be stem from blunted microglial activity, coupled with a reduction of oxidative and inflammatory mediators. Indeed, IFN- $\gamma$ deficiency was found to have neuroprotective 
consequences that were associated with a reduction of microglial activation (at least morphologically) and oxidative (e.g. iNOS and NADPH) and inflammatory (e.g. TNF-a, COX-2) factors. Moreover, paraquat was found to reduce trophic and pro-neuroplastic (BDNF, CREB) factors early in the course of treatment and IFN- $\gamma$ deficiency prevented this effect. These findings suggest that IFN- $\gamma$ may be a critical cytokine linking toxin exposure to microglia-driven pathological process and hence be an important therapeutic target in PD.

\section{Materials and methods}

Development of the IFN- $\gamma$ knockout (KO) mouse has been described previously (Dalton et al., 1993). IFN- $\gamma \mathrm{KO}$ and wild-type mice raised on a C57BL/6 genetic background were obtained from Jackson Laboratory (Bar Harbor, ME, USA) and interbred for successive generations. Homozygous wildtype and IFN- $\gamma$ KO littermates were subsequently generated from heterozygous breeding pairs. Between 8-10 weeks of age, males were single housed in standard polypropylene cages $(27 \times 21 \times 14 \mathrm{~cm})$, and maintained on a 12-hour light/dark cycle. Water and Ralston Purina mouse chow (St. Louis, MO) were provided ad libitum, and the room temperature maintained at $21^{\circ} \mathrm{C}$. All experimental test paradigms were approved by the Carleton University Committee for Animal Care and adhered to the guidelines outlined by the Canadian Council for the Use and Care of Animals in Research.

Experiment 1: Immunohistochemical assessment of SNc DA neuronal survival, as well as microglial morphology and expression of NADPH oxidase subunits following chronic paraquat exposure.

Intraperitoneal (i.p.) injections of paraquat (1,1'-dimethyl-4,4'-bipyridinium dichloride; Sigma-Aldrich, USA), at a dose of $10 \mathrm{mg} / \mathrm{kg}$, or an equivalent volume of 
physiological saline (Sigma-Aldrich), were administered to male IFN- $\gamma \mathrm{KO}$ and wildtype mice ( $n=10$ /group), times a week for 3 consecutive weeks ( 9 injections in total). This dose of paraquat has been utilized previously with minimal systemic toxicity (Brooks et al., 1999; Mangano and Hayley, 2009). Seven days following the final paraquat injection, mice were euthanized and perfused using 4\% paraformaldehyde (PFA). Excised brains were post-fixed in 4\% PFA for 24-hours and then cryoprotected for 3 days in a solution comprising of $20 \%$ sucrose, $0.1 \mathrm{M}$ PBS and $0.02 \%$ sodium azide ( $\mathrm{pH} 7.4$ ).

\section{Immunohistochemical procedures}

Frozen brains were cryostat-sectioned and coronal sections $(20 \mu \mathrm{m})$ obtained throughout the rostro-caudal axis of the SNc and mounted onto gelatin-coated slides. Loss of DA neurons was assessed using anti-tyrosine hydroxylase (TH) antibody and counterstained with $1 \%$ cresyl violet (Sigma-Aldrich). The primary antibody (TH) was diluted in a solution containing $1 \%$ BSA, $0.5 \%$ Triton $\mathrm{X}-100,0.05 \%$ Tween 20 and $0.05 \%$ sodium azide in $0.01 \mathrm{M}$ PBS, $\mathrm{pH} 7.3$ and the sections were incubated overnight at room temperature (1:3000, ImmunoStar, Hudson, WI,USA). The primary antibodies were visualized using a biotin rabbit anti-mouse (1:500, Cedarlane) secondary and horseradish peroxidase-conjugated streptavidin tertiary (1:500, Cedarlane). Sections were incubated with diaminobenzidine (DAB; Sigma-Aldrich) for 10 minutes, counterstained with cresyl violet (Sigma-Aldrich) and dehydrated with serial alcohol washes before cover slipping with clearene (Surgipath).

Survival of SNc DA-producing cells was determined by way of serial section analysis of the number of $\mathrm{TH}^{+}$cells within the $\mathrm{SNc}$, at bregma levels ranging from -2.70 to -3.88 . Using a double blind procedure 8-10 animals were analyzed per group and the 
total number of $\mathrm{TH}^{+}$cells was counted across multiple bregma levels. Midbrain sections from each bregma level and animal were compared across treatment groups. The same midbrain sections used to determine TH loss were used to validate TH loss. Specifically, the total number of neurons stained with cresyl violet from each bregma level and animal were averaged to determine total number of surviving neurons for each animal.

Changes in the cellular morphology of microglia, as indicated by differing degrees of compaction of the soma and thickening of the normally thin varicose processes, along with proliferative changes are taken to reflect a state of activation (Nimmerjahn et al., 2005). To this end, the morphological state of microglia was assessed using CD11b (complement receptor marker present on microglia, which we previously found to be a sensitive marker of microglial activation state; (Mangano and Hayley, 2009) anti-serum (1:1000; AbD Serotec, Raleigh, NC, USA; overnight incubation at ${ }^{4} \mathrm{C}$ ). Thereafter, sections were washed with $0.01 \mathrm{M}$ PBS and visualized by incubating with the appropriate AlexaFluor secondary antibody for 2-hrs at room temperature (1:200; Invitrogen, Carlsbad, CA, USA).

\section{Confocal Analysis}

Free-floating SNc sections $(20 \mu \mathrm{m})$ were double-labelled with rat anti-CD11b (1:1000, Serotec) and rabbit anti-gp91 ${ }^{\text {PHOX }}$ or $\mathrm{p}^{\text {PHOX }}$ (1:200; BD Transduction, Lexington, KY, USA). Enhanced expression of these NADPH oxidase subunits upon $\mathrm{CD}_{1} 1 \mathrm{~b}^{+}$microglial should reveal the potential ability of these cells to release the superoxide radical. Sections were washed 3 times with $0.01 \mathrm{M}$ PBS and blocked for 1-hr at room temperature with a $10 \%$ normal serum solution containing $1 \% \mathrm{BSA}, 0.5 \%$ Triton $\mathrm{X}-100,0.05 \%$ Tween 20 and $0.05 \%$ sodium azide in $0.01 \mathrm{M}$ PBS, $\mathrm{pH}$ 7.3. Sections were 
incubated with primary antibodies in the blocking solution overnight at $4^{\circ} \mathrm{C}$. All antibodies were incubated in a solution containing $1 \% \mathrm{BSA}, 0.5 \%$ TritonX-100, $0.05 \%$ Tween 20, and visualized using appropriate secondary AlexaFluro antibody

(1:200; Invitrogen) following 2-hrs incubation at room temperature. Co-localization was determined using EZ-C1 software with a Nikon C1 confocal system.

Experiment 2: Time-dependent changes in the expression of inflammatory, oxidative and trophic factors induced by paraquat.

A separate time course was conducted to ascertain the temporal nature of paraquat induced alterations of oxidative and inflammatory signalling factors thought to underlie DA neuronal death, as well as select trophic factors (e.g. BDNF) hypothesized to counteract such inflammatory and degenerative processes. IFN- $\gamma \mathrm{KO}$ and wildtype littermate mice were randomly assigned to paraquat $(10 \mathrm{mg} / \mathrm{kg}$; i.p.) or saline $(n=$ 8/group) treatment groups. However, in this experiment, injections were administered 3 times per week for either 1 or 3 weeks. Animals were subsequently euthanized by rapid decapitation 2-hrs following their final injection, the SNc and striatum were removed for processing by quantitative real-time PCR. A 2-hr sacrifice interval was chosen in light of our previous work showing that alterations in brain regional neurochemistry and cytokines are most robust within this time frame (Litteljohn et al., 2009; Mangano and Hayley, 2009).

\section{Brain dissection technique}

2-hrs after the final treatment (i.e. post- $3^{\text {rd }}$ or $-9^{\text {th }}$ injections), sequential coronal slices were collected using razor blades and a chilled stainless steel microdissecting block with adjacent slots arranged $1.0 \mathrm{~mm}$ apart. As previously described (Hayley et al., 2004b), SNc and striatal nuclei were collected by micropunch, with reference to the 
mouse brain atlas of Franklin and Paxinos (1997). Tissue samples were stored at $-80{ }^{\circ} \mathrm{C}$ until later PCR processing.

\section{Quantitative Real-time PCR array}

A custom PCR array comprising 23 oxidative, inflammatory and trophic genes of interest, 3 housekeeping genes, as well as several internal controls was purchased from SABiosciences (Frederick, MD, USA). All of the genes assayed are listed in Table 1. All primers were designed and optimized by SABiosciences. In order to ensure the purity and quality of tissue samples, only those which met the genomic contamination criteria, reverse transcription control, and positive PCR control set out by SABioscience were used during the data analysis.

Unless specified, all reagents and kits were purchased from SABioscience; detailed protocols can be found on the company's website at www.sabiosciences.com. Briefly, Trizol (Invitrogen, Burlington, ON, Canada) was used to isolate RNA from SNc and striatum tissue samples, according to the manufacturer's instructions. RNA was subsequently purified by passing the samples through a series of spin columns and treatment with DNAse (PA-001). The quality of the RNA was determined using Experion $^{\text {TM }}$ RNA highSens Analysis kit (BioRad Laboratories, Hercules, CA, USA); only samples with RNA quality indicator (RQI) values between 7.2 and 9.0 were used for qPCR. After treating samples with a genomic DNA elimination mixture (to prevent genomic contamination), a Nano PreAMP cDNA synthesis kit was used (C-06) to create first strand cDNA. SYBR green mixture was used according to the manufacturer's protocol for the $\mathrm{qPCR}$, and samples were analyzed with a BioRad iQ5 real-time thermocycler. 
The mRNA expression of each gene of interest was normalized by subtracting the average of the $C_{t}$ values of the housekeeping genes from the gene of interest's $C_{t}$ [i.e., $\Delta C_{t}=C_{t}$ (gene of interest) $-C_{t}$ (housekeeping genes)]. The $2^{-\Delta \Delta C t}$ method was subsequently used to convert $\Delta \mathrm{C}_{\mathrm{t}}$ values to mRNA fold changes relative to the control group, as described elsewhere (Livak and Schmittgen 2001; Schmittgen and Livak 2008).

Table 1. SA Bioscience Gene List

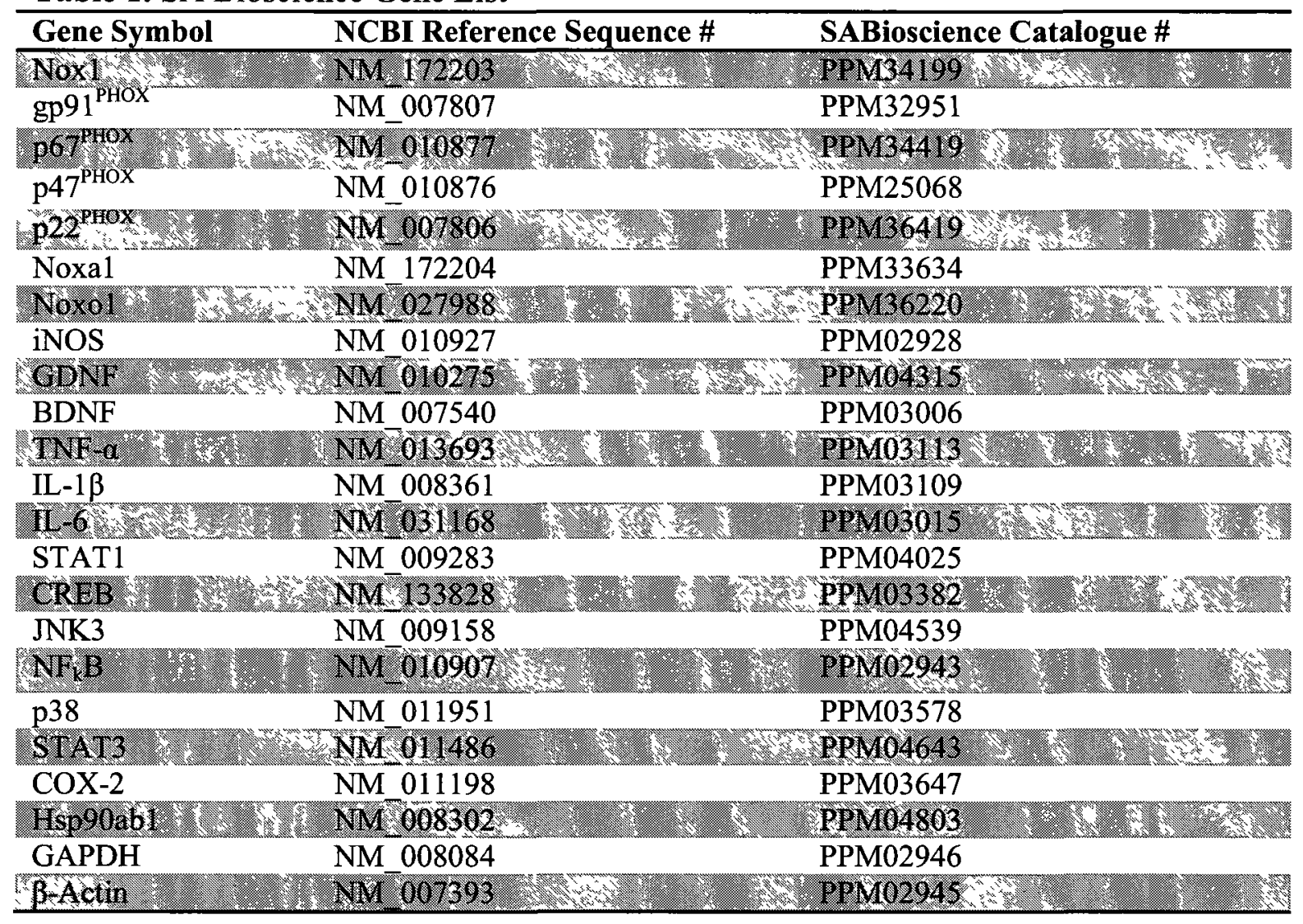

\section{HPLC determination of paraquat accumulation}

In order to determine whether genotype influenced the entry and accumulation of paraquat in specific brain regions a separate set of wild type $(n=9)$ and IFN- $\gamma$ KO $(n=6)$ mice were subjected to the same paraquat injection regimen of the previous studies. 
Following 3 weeks of paraquat dosing ( 3 times $/$ week; $10 \mathrm{mg} / \mathrm{kg}$; i.p.), brains were rapidly removed after decapitation. Brains were placed cortex side down in a stainless steel brain blocker and razor blades inserted to produce $2.0 \mathrm{~mm}$ coronal tissue sections. Thereafter, striatal and SNc brain regions were collected by micropunch (as previously described in Hayley et al., 1999; Hayley et al., 2004). All brain samples were taken with reference to the mouse brain atlas of Franklin and Paxinos (1997) and were stored at $-80^{\circ} \mathrm{C}$ until processing.

Paraquat accumulation within the brain was determined through highperformance liquid chromatography (HPLC) as previously described (Hayley et al. 1999). Briefly, several brain regions (cortex, hippocampus, striatum and midbrain) were sonicated for 20 minutes in a solution containing $12 \%$ acetic acid (few seconds by sonic dismembrator followed by 20 minutes in ultrasound water bath). The tissue samples were further centrifuged for 10 minutes at $21000 \mathrm{RCF}$ and supernatant transferred to HPLC /auto-sample vials. After centrifugation $50 \mu \mathrm{l}$ of sample was injected into the HPLC system and paraquat separation was carried at a flow rate of $1.5 \mathrm{ml} / \mathrm{min}$ through a system equipped with a ZORBAX SB-C8 column, a radial compression column (4.6um $\mathrm{x}$ $250 \mathrm{~mm}$; Agilant Technologies), using a UV detector $(263 \mathrm{~nm})$. The mobile phase used for separation comprised of $3.5 \mathrm{ml}$ tri ethylamine, $1.0 \mathrm{ml}$ 1-hexane-sulphonic acid, sodium salt and D1 $800 \mathrm{ml} \mathrm{H} 2 \mathrm{O}$ (HPLC grade) that had been filtered using 0.22-mm filter paper, degassed, and the $\mathrm{pH}$ levels adjusted to 2.5 using ortophosphoric acid. Stock solution

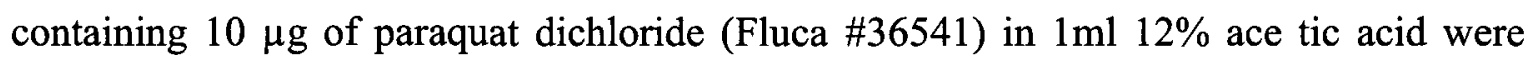
used to determine to total amount of paraquat accumulation within the brain. 
A Hewlett-Packard integrator determined the height and area of the peaks. The protein content of each sample was measured using bicinchoninic acid with a protein analysis kit (Pierce Scientific, Canada), and a spectrophotometer (Brinkman, PC800 colorimeter). Paraquat determination was based on protein levels.

\section{Statistical Analyses}

All data were analysed by ANOVA followed by Fisher's planned comparisons where appropriate (unless otherwise stated). Data were evaluated using a StatView (version 6.0) statistical software package available from the SAS Institute, Inc.

\section{Results}

IFN- $\gamma$ deficiency prevented dopamine neuron loss following paraquat treatment

Consistent with our hypothesized role of IFN- $\gamma$ in the neurodegenerative effects of paraquat, the impact of the pesticide was abrogated in mice lacking the cytokine. Specifically, the number of surviving $\mathrm{TH}^{+}$neurons varied as a function of IFN- $\gamma$ deletion and paraquat treatment at $\mathrm{SNc}$ levels corresponding to bregma levels $-3.08,-3.16$, and $3.28\left\{F_{\mathrm{S}_{1,24}}=44.46,10.19\right.$ and 6.219 , respectively, $\left.p<0.05\right\}$. As shown in Figure 1, the three week paraquat regimen provoked a moderate $(\sim 35 \%)$ but statistically significant reduction in $\mathrm{TH}^{+}$neurons in wildtype mice across the three SNc levels $(p<0.05)$. However, this effect was completely absent in the IFN- $\gamma$ deficient mice (Figure 2.1). In order to ascertain whether the loss of $\mathrm{TH}^{+}$staining reflects an actual loss of neurons or is simply a phenotypic suppression of the enzyme, we quantified counterstained cresyl violet $\mathrm{TH}^{+}$and $\mathrm{TH}^{-}$neurons within the $\mathrm{SNc}$. To this end, we found that paraquat provoked a selective loss of $\mathrm{TH}^{+}$cresyl violet stained $\mathrm{SNc}$ neurons (in wildtype animals), whereas no difference was observed in $\mathrm{TH}^{-}$cresyl violet neurons (data not shown). 
However, the overall number of cresyl violet neurons within the SNc was reduced, indicating that there was a genuine neuronal loss induced. Moreover, the fact that paraquat only influenced $\mathrm{TH}^{+}$cresyl violet counterstained neurons indicates that this cellular population is selectively vulnerable to the neurodestructive effects of the pesticide.

Insert Figure 2.1 about here

Accumulation of paraquat within the brain

Accumulation of paraquat was measured within the striatum and midbrain of wild type and IFN- $\gamma$ KO mice to determine if genetic deletion of the cytokine differentially affected the central accumulation and regional distribution of the herbicide. To this end, mice exposed to paraquat $3 \times /$ week for 3 weeks had comparable levels of paraquat within the two brain regions analyzed. Specifically, the mean values of paraquat accumulation within the striatum and $\mathrm{SNc}$ were $3.689 \pm 0.472$ and $4.58 \pm 0.57 \mathrm{ng} / \mathrm{mg}$ tissue, respectively $(\mathrm{n}=9)$ for the wild type mice and $4.933 \pm 0.58$ and $4.93 \pm 0.41 \mathrm{ng} / \mathrm{mg}$ tissue, respectively $(\mathrm{n}=6)$ for the IFN- $\gamma$ KO mice. These were not significantly different in either case (t-tests, $\mathrm{p}>0.05$ ). Thus, IFN- $\gamma$ deletion clearly did not affect midbrain accumulation of paraquat and the very slight (but not significant) elevation in paraquat in the striatum of knockout mice should certainly not explain the neuroprotective consequences observed in these animals. 


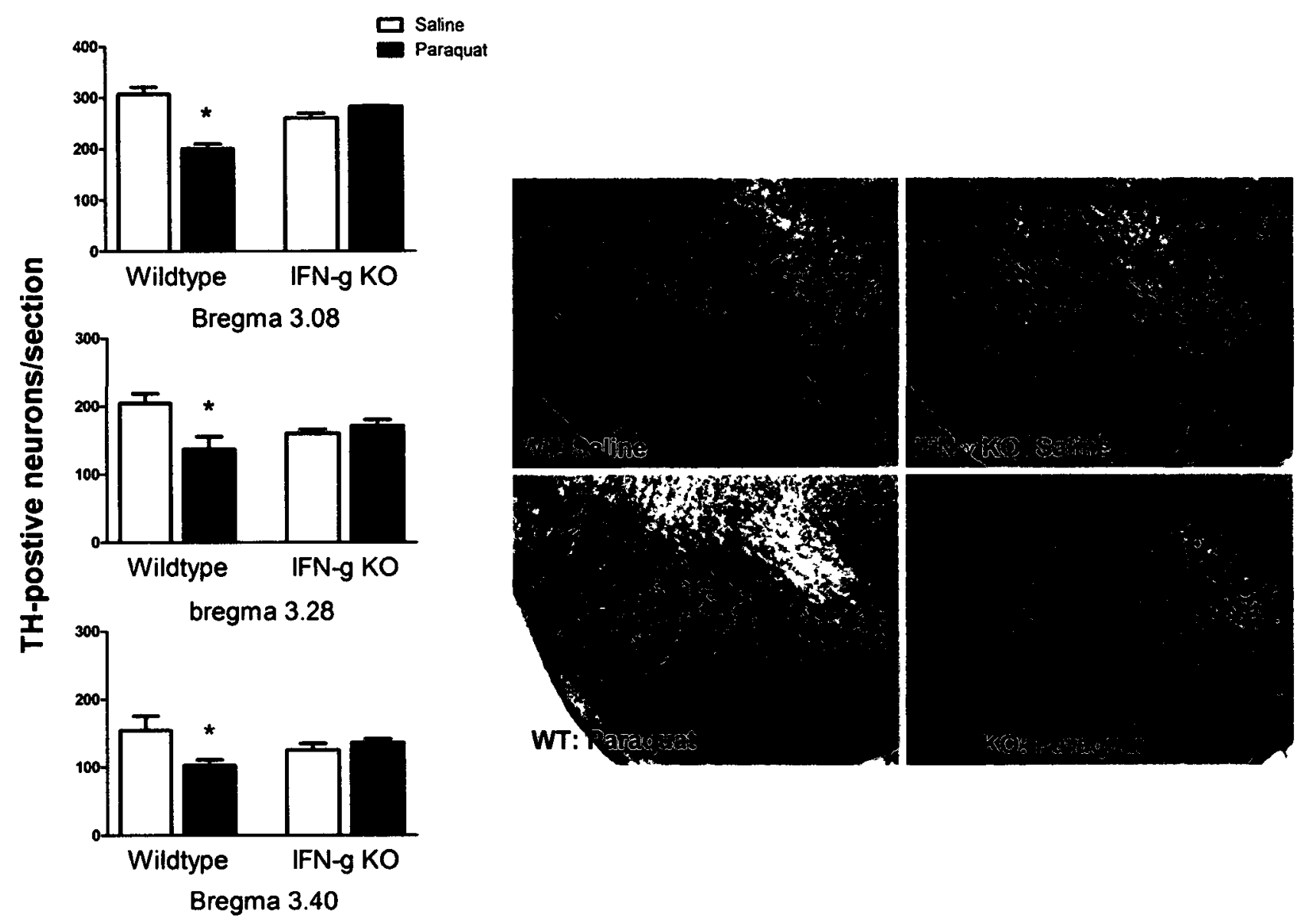

Figure 2.1 IFN- $\gamma$ KO mice were resistant to paraquat toxicity.

As shown in the representative photomicrographs on the right, paraquat exposure $(10 \mathrm{mg} / \mathrm{kg}$, three times a week for three weeks) provoked a significant reduction in $\mathrm{TH}^{+}$neurons of wildtype (WT) mice but had no such effect on IFN- $\gamma$ KO animals. The left bar graphs display the quantification of $\mathrm{TH}^{+}$neuronal loss across three different $\mathrm{SNc}$ bregma levels. Error bars represent SEM, 2-way ANOVA * $\mathrm{p}<0.001$ 
Paraquat increased morphological signs of microglial reactivity and pro-inflammatory cytokine expression in wild-type but not IFN- $\gamma$ null mice

As previously observed (Mangano and Hayley, 2009), paraquat increased staining of the microglial cell marker, CD11b, within the SNc of wildtype mice. These microglial cells displayed morphological characteristics indicative of an intermediately activated state, including short, thickened dendritic processes and a bushy rounded soma (see representative photomicrographs in Figure 2.2). In contrast, no differences were observed between paraquat treated IFN- $\gamma \mathrm{KO}$ mice and their saline-treated counterparts with respect to $\mathrm{CD}_{11 \mathrm{~b}^{+}}$staining. In all instances, $\mathrm{CD} 11 \mathrm{~b}^{+}$cells from IFN- $\gamma \mathrm{KO}$ mice displayed thin, highly ramified branching patterns and fine, less obvious soma indicative of a microglial "resting" state (Nimmerjahn et al., 2005). Hence, the morphological state of microglia, which is intimately linked to the inflammatory potential of these cells, was clearly associated with the degree of degeneration observed within the SNc.

Insert Figure 2.2 about here

Pro-inflammatory cytokines may contribute to the neurodegenerative response induced by paraquat by promoting a heightened state of microglial reactivity and inducing the release of oxidative species. In the present investigation, paraquat timedependently provoked changes in the expression of several pro-inflammatory cytokines within the SNc. As shown in Figure 2.2, the pro-inflammatory cytokines, TNF- $\alpha$ and IL$1 \beta$, displayed similar temporal expression patterns. Specifically, a transient albeit nonsignificant reduction in cytokine mRNA was evident after 1 week (data not shown). In contrast, at week 3, separate ANOVAs revealed a significant Genotype $\times$ Treatment 

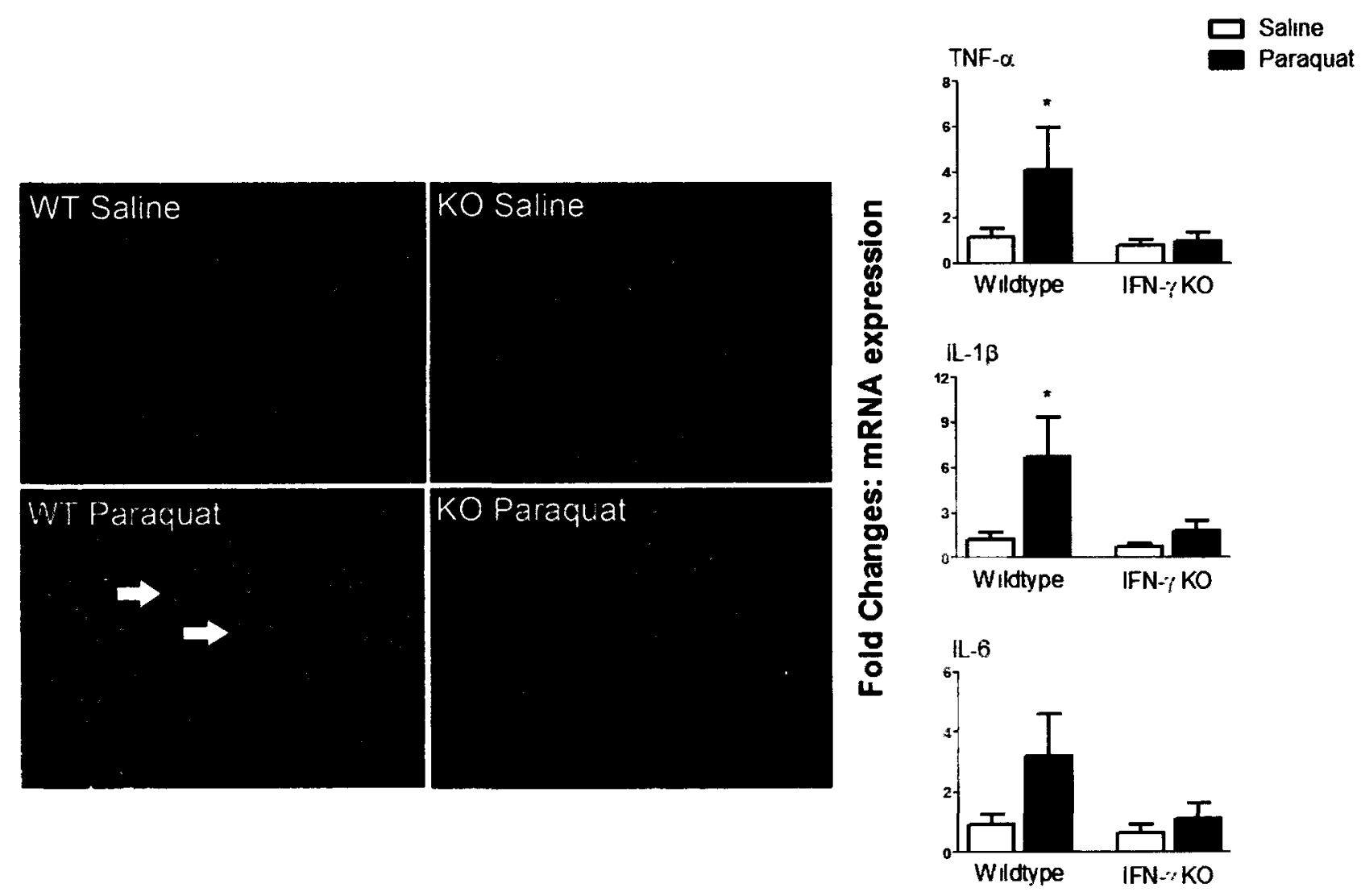

Figure 2.2 IFN- $\gamma$ deficiency prevented paraquat induced activation of microglia.

Representative photomicrographs of CD11b immunostaining within the SNc of wildtype (WT) and IFN- $\gamma$ knockout (KO) mice exposed to either saline or paraquat are depicted on the left. Arrows indicate microglial cells with morphological changes typical of a state of intermediate level reactivity. The right hand bar graphs reveal the quantification of expression of TNF- $\alpha$, IL-6 and IL- $1 \beta$ mRNA measured using q-PCR from a separate group of saline or paraquat treated WT and IFN- $\gamma$ KO mice. Results are mean \pm SEM, 2-way ANOVA * $\mathrm{p}<0.05$. 
interaction for both TNF- $\alpha$ and IL-1 $\beta\left\{F_{\mathrm{S}_{1,17}}=5.369\right.$ and 5.587, respectively, $\left.p<0.05\right\}$. As shown in Figure 2.2 and confirmed by the follow-up comparisons, paraquat markedly increased TNF- $\alpha$ and IL-1 $\beta$ expression within the SNc of wildtype animals after 3 weeks $(p<0.05)$. However, mice lacking IFN- $\gamma$ were entirely protected against these lateroccurring pesticide-induced effects. In contrast to TNF- $\alpha$ and IL-1 $\beta$, mRNA expression of IL- 6 was not significantly altered after 3 weeks of paraquat treatment among mice of either genotype. Yet, as shown in Figure 2.2, there was a definite trend towards an elevation of IL-6 expression; and, once again, this effect (albeit non-significant) was largely restricted to the wildtype mice.

Relative to the SNc, the striatum appeared to be far less susceptible to the impact of paraquat on brain cytokine mRNA expression levels, at least at the time-points (and sacrifice intervals) assayed. Indeed, the ANOVAs failed to reveal any significant main effects of or interactions between Genotype and Treatment for any of the cytokines at either of the experimental time-points (data not shown).

\section{IFN- $\gamma$ deficiency attenuated NADPH oxidase activation following paraquat exposure}

Paraquat time-dependently increased the SNc mRNA expression of several subunits of the pro-oxidant enzyme, NADPH oxidase. This complex inducible enzyme is located on the outer membrane of microglia and requires cytosol-to-membrane translocation of a subset of the enzymatic subunits to generate superoxide radicals (Qian et al., 2009; Qian et al., 2008). At the end of week 1, no changes were detected in mRNA expression of the subunits making up either the phagocytic (gp91 ${ }^{\mathrm{PHOX}}, \mathrm{p} 47^{\mathrm{PHOX}}$ and p6 $7^{\text {PHOX }}$ ) or non-phagocytic (Noxo1, Nox1 and Noxa1) isoforms of NADPH oxidase among wildtype or IFN- $\gamma \mathrm{KO}$ mice (data not shown). However, by week 3 , all of the 
subunits varied as a function of paraquat treatment, and most of these effects were influenced by IFN- $\gamma$ deficiency.

Indeed, at week 3, paraquat caused robust IFN- $\gamma$-dependent changes in the mRNA expression of each of the phagocytic NADPH oxidase subunits assayed (Figure 3, leftmost panel). Specifically, the ANOVAs revealed a significant Genotype $\times$ Treatment interaction for both membrane-bound ( $\mathrm{p} 22$ and $\mathrm{gp} 91^{\mathrm{PHOX}}$ ) and cytosolic ( $\mathrm{p} 47^{\mathrm{PHOX}}$, p67 ${ }^{\text {PHOX }}$ ) enzymatic subunits $\{F \mathrm{~s} 1,17=4.28,19.25,2.75$ and 11.43 , respectively, $p<$ $0.05\}$. In each case, mRNA concentration was significantly elevated in the SNc among wildtype mice following pesticide treatment $(p<0.05)$. Conversely, as shown in Figure 3 , the IFN- $\gamma \mathrm{KO}$ mice were completely resistant to these paraquat-induced effects. It is important to underscore that the elevations of these oxidative subunits only occurred at week 3, paralleling paraquat's maximal neurodegenerative effects. Indeed, we found that paraquat did not provoke a significant loss of $\mathrm{TH}^{+} \mathrm{SNc}$ neurons at earlier time-points (e.g., after 1 week of treatment).

Insert Figure 2.3 about here

Consistent with the fact that NADPH oxidase is robustly expressed within microglia, both the catalytic (gp91 ${ }^{\mathrm{PHOX}}$ ) and inducible cytosolic $\left(\mathrm{p} 67^{\mathrm{PHOX}}\right)$ subunits were found to be co-localized in $\mathrm{CD} 11 \mathrm{~b}^{+}$cells within the SNc (Figure 2.3). Paralleling the mRNA data, immunohistochemical analyses revealed that the enzymatic subunits were selectively increased in $\mathrm{CD}_{11 \mathrm{~b}^{+}}$microglia within the SNc of paraquat-treated wildtype but not IFN- $\gamma$ KO mice (Figure 2.3). 
Figure 2.3 Paraquat elevated the expression of several NADPH oxidase subunits within the SNc of wild type (WT) but not IFN- $\gamma$ knockout (KO) mice.

The bar graphs shown on top panels (A) reveal mRNA for cytosolic (p67pHox, p47pHox, Noxa1, Noxo1) and membrane-bound (p22PHox, gp91PHox, Nox1) subunits of NADPH oxidase was measured by q-PCR in wildtype and IFN- $\gamma \mathrm{KO}$ mice after 3 week exposure to either saline or paraquat. The data depict mean fold changes \pm SEM relative to housekeeping genes. ${ }^{*} p<0.0001,2$-way ANOVA. The bottom representative photomicrographs display staining for the microglial marker, CD11b (green) and either the gp91 PHOX (top panel) or p67PHox (bottom panel) NADPH oxidase subunits (red). Paraquat treatment induced gp91PHox and p67PHox staining in WT mice (panels D and E, respectively) and these subunits were clearly co-localized upon microglia (yellow region of overlap indicated by arrows). In contrast, IFN- $\gamma$ KO mice treated with paraquat (panels $\mathrm{H}$ and I) had virtually undetectable levels of the NADPH oxidase subunits and were indistinguishable from either saline treated WT (panels B and C) or IFN- $\gamma$ KOs (panels F and $\mathrm{G})$. 


\section{A SNc: Week 3}
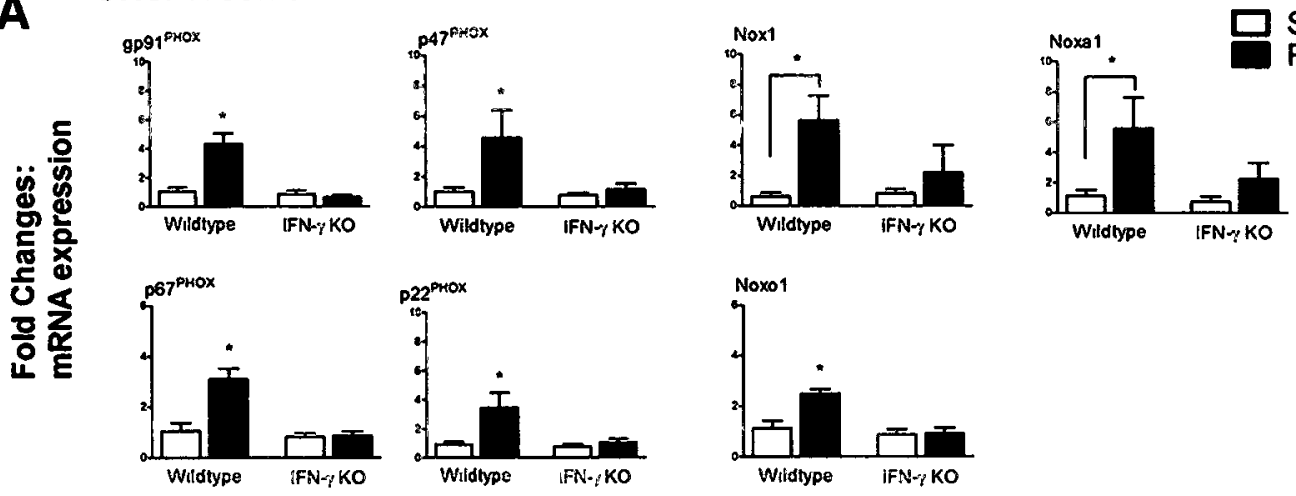

Saline

Paraquat
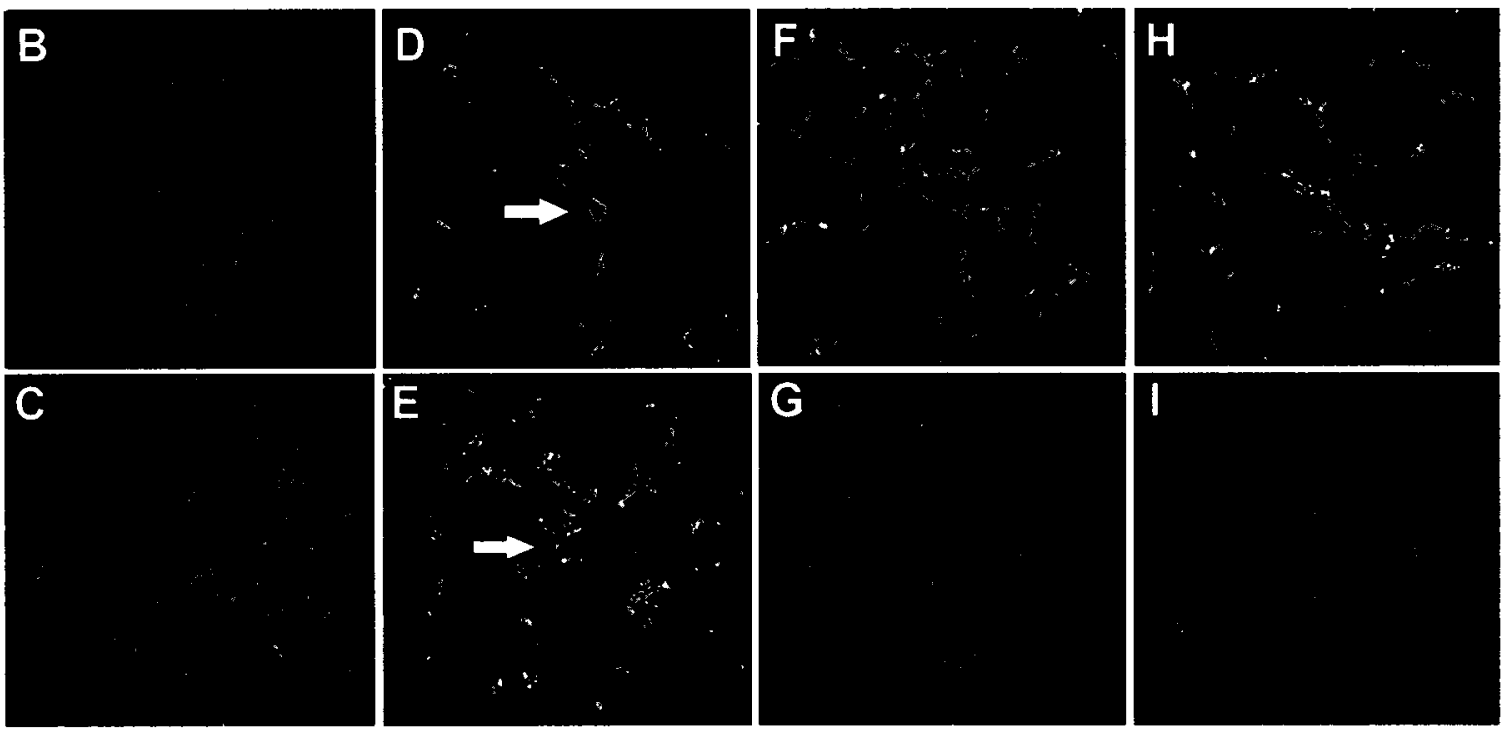
With respect to the non-phagocytic subunit isoforms of NADPH oxidase (i.e., Noxo1, Nox1 and Noxa1), similar patterns of SNc mRNA expression was comparable to the aforementioned phagocytic NADPH oxidase. Indeed, while Noxo1, Nox1 and Noxa1 were not altered by the experimental manipulations at the early time-point (week 1); by week 3, paraquat differentially influenced mRNA expression levels of these subunits among wildtype and IFN- $\gamma$ KO animals. Specifically, the ANOVA revealed a significant Genotype $\times$ Treatment interaction for Noxo1 $\left\{F_{1,16}=7.313 p<0.05\right\}$, such that paraquat increased levels of this cytosolic subunit within the SNc of wildtype mice $(p<0.05)$, but not among the IFN- $\gamma$ KO (Figure 3). Similarly, by week 3, mRNA expression for both the catalytic membrane-bound subunit, Nox1 (gp91 ${ }^{\text {PHOX }}$ homolog), and the cytosolic Noxa1 subunit (p67 $7^{\text {PHOX }}$ homolog) were significantly augmented following paraquat treatment $\left\{F \mathrm{~s}_{1,16}=6.74\right.$ and 5.945, respectively $\left.p<0.05\right\}$. Although the ANOVAs failed to reveal a significant interaction effect of Genotype with Treatment for the SNc mRNA levels of these two subunits, the paraquat-induced mRNA changes appeared to be largely restricted to wildtype animals. Indeed, as shown in Figure 3, IFN- $\gamma$ deficiency clearly attenuated the rise in Nox1 and Noxal evident in the SNc of wildtype mice $(p<0.05)$.

As was the case for the cytokine variations, very few changes in NADPH oxidase subunit mRNA expression were detected within the striatum (data not shown). In fact, only $\mathrm{p} 47^{\mathrm{PHOX}}$ was significantly affected by the pesticide $\left\{F_{1,23}=4.487 p<0.05\right\}$, such that striatal mRNA expression levels of this subunit were elevated following 3 weeks of paraquat treatment, irrespective of IFN- $\gamma$ deficiency.

Paraquat-induced changes in MAP kinase expression were prevented by IFN- $\gamma$ knockout The MAP kinases, p38 $\alpha$ and JNK3 have been implicated in toxin-induced neurodegeneration (Choi et al., 2010; Newhouse et al., 2004; Peng et al., 2004). Here, we 
report that paraquat induced robust time- and- IFN- $\gamma$-dependent variations of MAP kinase expression in the SNc but not striatum. In this regard, within the SNc, neither of the MAP kinases was significantly affected by paraquat at week 1, although the IFN- $\gamma$ KOs appeared to display increased JNK3 levels at this early time-point (Figure 2.4). Contrastingly, at week 3, separate ANOVAs revealed that SNc mRNA expression of both p38 $\alpha$ and JNK3 significantly varied according to the interaction of Genotype with Treatment $\left\{F_{\mathrm{S}_{1,17}}=5.360\right.$ and 5.959 , respectively, $\left.p<0.05\right\}$. While paraquat increased mRNA expression of both MAP kinases in wildtype animals $(p<0.05)$, these effects were absent among the IFN- $\gamma$ KO mice (Figure 2.4). Thus, p38 $\alpha$ and JNK3 not only followed similar SNc expression patterns over time in response to the paraquat treatment and IFN- $\gamma$ deletion, but also varied analogously with the aforementioned proinflammatory cytokine and NADPH oxidase subunit expression levels within this same brain region.

Insert Figure 2.4 about here

Paraquat provoked time- and-IFN- $\gamma$-dependent variations of inflammatory transcription factors and their downstream enzymes

In addition to changes in MAP kinase expression, it was of interest to determine the time-dependent influence of paraquat on several key transcription factors involved in inflammation and/or IFN- $\gamma$ signalling. In this regard, the pesticide robustly affected SNc expression of both STAT1 (a primary mediator of IFN- $\gamma$-regulated transcription) and NFאB (but not STAT3), and once again, these changes were further influenced by time (i.e., week 1 vs. week 3) and IFN- $\gamma$ deletion. 


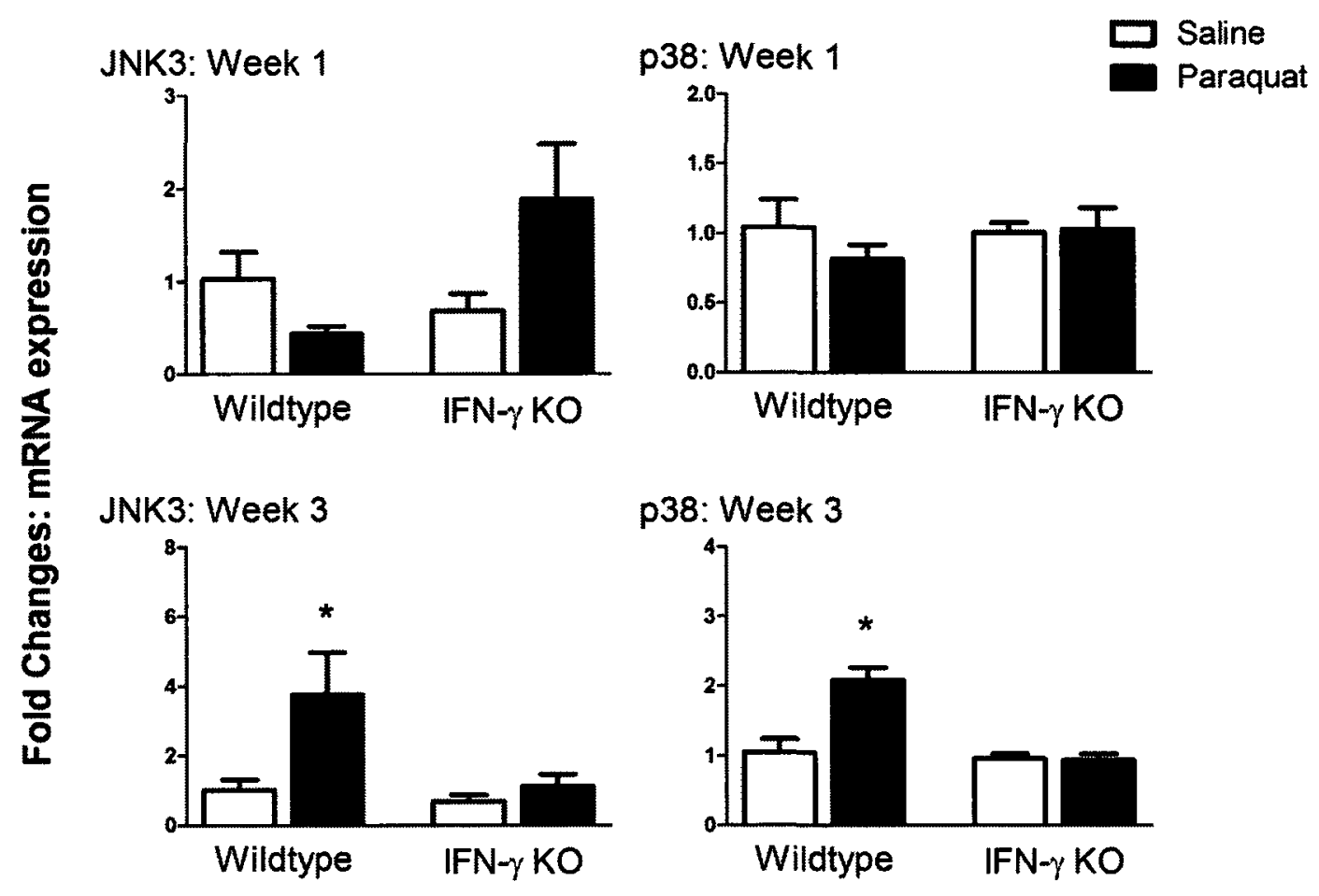

Figure 2.4 Paraquat-induced elevations of SNc mRNA of the MAP kinases, JNK3 and p38, were absent in IFN- $\gamma$ deficient mice.

Expression of mRNA of JNK3 and p38 was assessed using qPCR in wildtype and IFN- $\gamma$ KO mice after 1 (top panels) and 3 (bottom panels) weeks of exposure to either saline (white bars) or paraquat (black bars). Results are presented as mean fold changes \pm SEM relative to that of housekeeping genes. ${ }^{*} \mathrm{p}<0.05,2$-way ANOVA. 
With respect to STAT1, separate ANOVAs revealed that paraquat significantly affected SNc mRNA levels of the transcription factor after both 3 (week 1) and 9 injections week 3 ; and IFN- $\gamma$ KO differentially modified these effects $\left\{F_{1,20}=3.342\right.$ and $F_{1,17}=7.478$, respectively, $\left.p<0.05\right\}$. As shown in Figure 2.5 and confirmed by the multiple comparisons, at week 1, paraquat (somewhat surprisingly) increased STAT1 expression within the SNc among IFN- $\gamma$ KOs but not wildtype mice $(p<0.05)$. Yet, by the end of week 3 , levels of the IFN- $\gamma$-activated transcription factor normalized among the paraquat-treated IFN- $\gamma$ deficient mice. Moreover, at this later time-point, wildtype mice treated with the pesticide displayed robustly elevated STAT1 expression $(\mathrm{p}<0.05)$.

Insert Figure 2.5 about here

Unlike STAT1, SNc mRNA expression of NFkB was not influenced by paraquat or IFN$\gamma$ deletion at the early week-1 time-point (data not shown); however, by week $3, \mathrm{NF}$ B levels were found to vary. Indeed, the ANOVA revealed a significant Genotype $\times$ Treatment interaction for NFאB $\left\{F_{1,17}=5.81, p<0.05\right\}$, such that paraquat increased SNc expression of this transcription factor, but once again, only in wildtype mice $(p<0.05)$. In contrast, SNc expression of STAT3, which is a primary mediator of IL-6 signalling (Murray, 2007), was completely unaffected by IFN- $\gamma$ deletion and paraquat treatment, at either of the time-points tested (data not shown).

The inflammatory enzymes, COX-2 and iNOS, which are downstream targets of STAT1 and NFkB (Bolli et al., 2003; de Prati et al., 2005; Tsatsanis et al., 2006), play a role in the degenerative process in toxin-based animal models of PD 
Proinflammatory cytokines and Parkinson's disease, 75

Figure 2.5 Paraquat-induced elevations in the transcription factors, STAT1 and NFkB, as well as the inducible enzymes, iNOS and COX-2 are modulated by IFN- $\gamma$ deficiency. The top and bottom series of bar graphs display mRNA changes (after 1 and 3 weeks, respectively) in wildtype and IFN- $\gamma$ KOs after treatment with either saline (white bars) or paraquat (black bars). Data presented are mean fold changes \pm SEM relative to that of housekeeping genes. ${ }^{*} \mathrm{p}<0.05,2$-way ANOVA. 
Fold Changes: mRNA expression
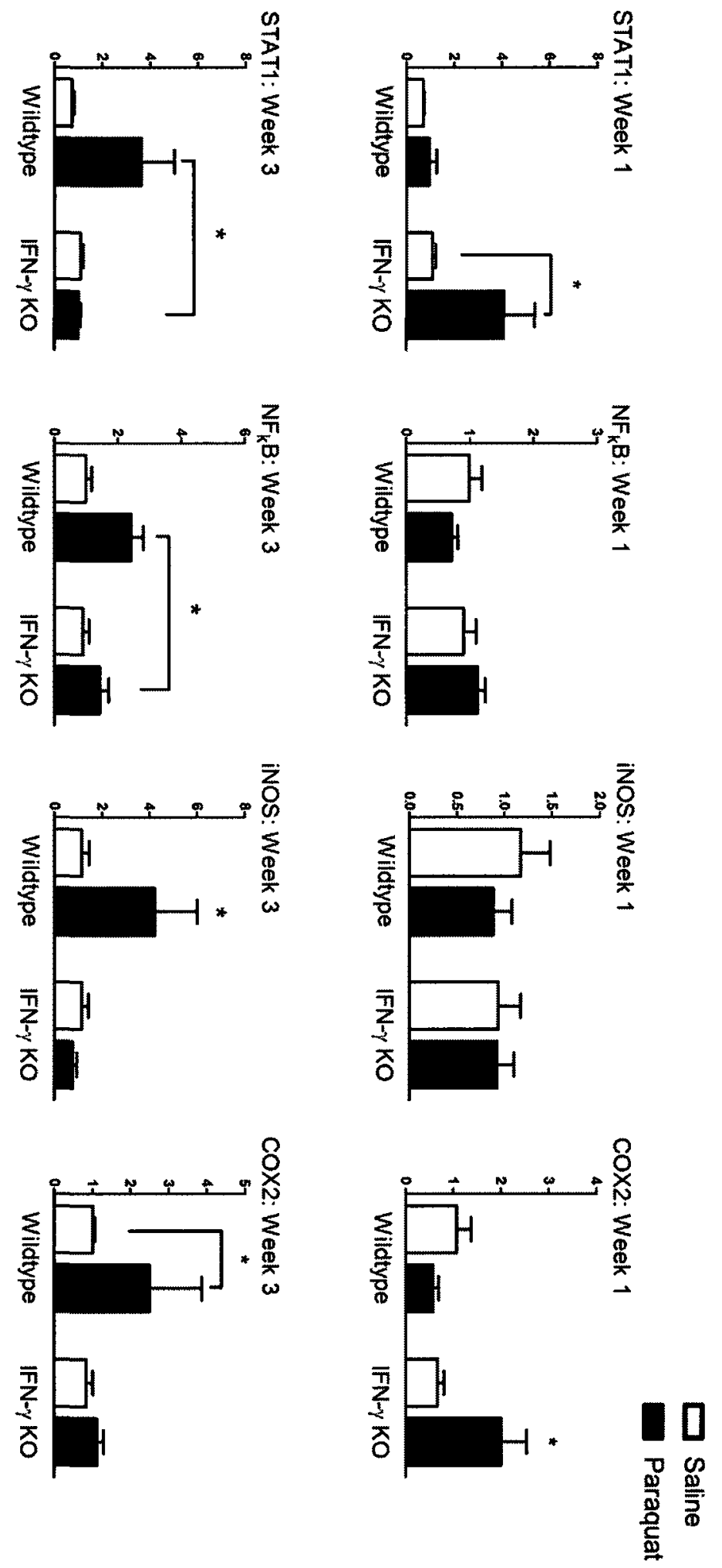
(Okuno et al., 2005; Wang et al., 2009; Gupta et al., 2010). Perhaps not surprisingly then, in the present study, both COX-2 and iNOS varied time-dependently in the SNc as a function of IFN- $\gamma$ deletion after paraquat treatment, although the enzymes displayed somewhat divergent expression patterns over time (again, no significant effects were observed in the striatum). In this regard, while no between-groups differences were observed for week-1 iNOS levels, COX-2 expression varied significantly at week 1 as a function of the interaction between Genotype and Treatment $\left\{F_{1,20}=4.88, p<0.05\right\}$. Much like STAT1, the follow-up comparisons revealed that paraquat caused an elevation of COX-2 among IFN- $\gamma$-deficient $(p<0.05)$, but not wildtype mice (see Figure 2.5 ).

In contrast, by the end of week 3 , separate ANOVAs revealed that paraquat and IFN- $\gamma$ deletion jointly influenced mRNA expression of both COX-2 and iNOS $\left\{F \mathbf{S}_{1_{17}}=\right.$ 5.816 and 4.958, respectively, $p<0.05\}$. As shown in Figure 2.5 and confirmed by the multiple comparisons, paraquat-treated wildtype mice displayed markedly enhanced COX-2 and iNOS expression relative to control animals $(p<0.05)$; however, IFN- $\gamma$ KO mice were completely unaffected by the pesticide treatment.

Paraquat time-dependently diminished CREB and BDNF levels within the SNc among wildtype but not IFN-y null mice

Besides oxidative and inflammatory mechanisms, there is reason to believe that reductions of trophic factors, stemming from alterations in cell survival signalling, may play a role in PD and animal models of the disease (Nagatsu et al., 2000; Parain et al., 1999). Accordingly, we assessed the impact of paraquat and IFN- $\gamma$ deletion on the CREB transcription factor and its downstream target, BDNF, within the SNc and striatum. Once again, no changes were observed within the striatum at either of the time-points assayed (data not shown). 
However, within the SNc, separate ANOVAs revealed that, at the early 1 week time-point, both CREB and BDNF varied significantly as a function of the interaction between Genotype and Treatment $\left\{F_{\mathbf{S}_{1,17}}=5.515\right.$ and 5.366, respectively, $\left.p<0.05\right\}$. Indeed, after 1 week of injections, paraquat-treated wildtype mice displayed diminished mRNA expression levels of both CREB and BDNF $(p<0.05$, relative to saline-treated wildtype controls), whereas IFN- $\gamma$ KO mice were completely resistant to such changes (Figure 2.6).

Insert Figure 2.6 about here

Unlike the majority of the pro-inflammatory and pro-oxidant factors assayed, CREB and BDNF mRNA expression levels were not significantly affected by the paraquat treatment at week 3 among mice of either genotype $(p>0.05)$. Indeed, as shown in Figure 2.6, the (transient) paraquat-induced week 1 reduction of SNc CREB and BDNF in wildtype animals had completely normalized by the end of week 3 .

\section{Discussion}

Accumulating evidence has implicated neuroinflammatory cascades in the death of midbrain DA neurons in PD patients and animal models of the disease (Hunot et al. 1999; Block and Hong, 2005; Miller et al., 2009). In this regard, numerous studies have demonstrated that SNc DA neurons are particularly vulnerable to the impact of inflammatory insults, such as LPS, as well as other toxins (e.g., MPTP, paraquat, and rotenone) that have been shown to stimulate inflammatory factors (Gao et al., 2003b; Thomas et al., 2008; Mangano and Hayley, 2009). 

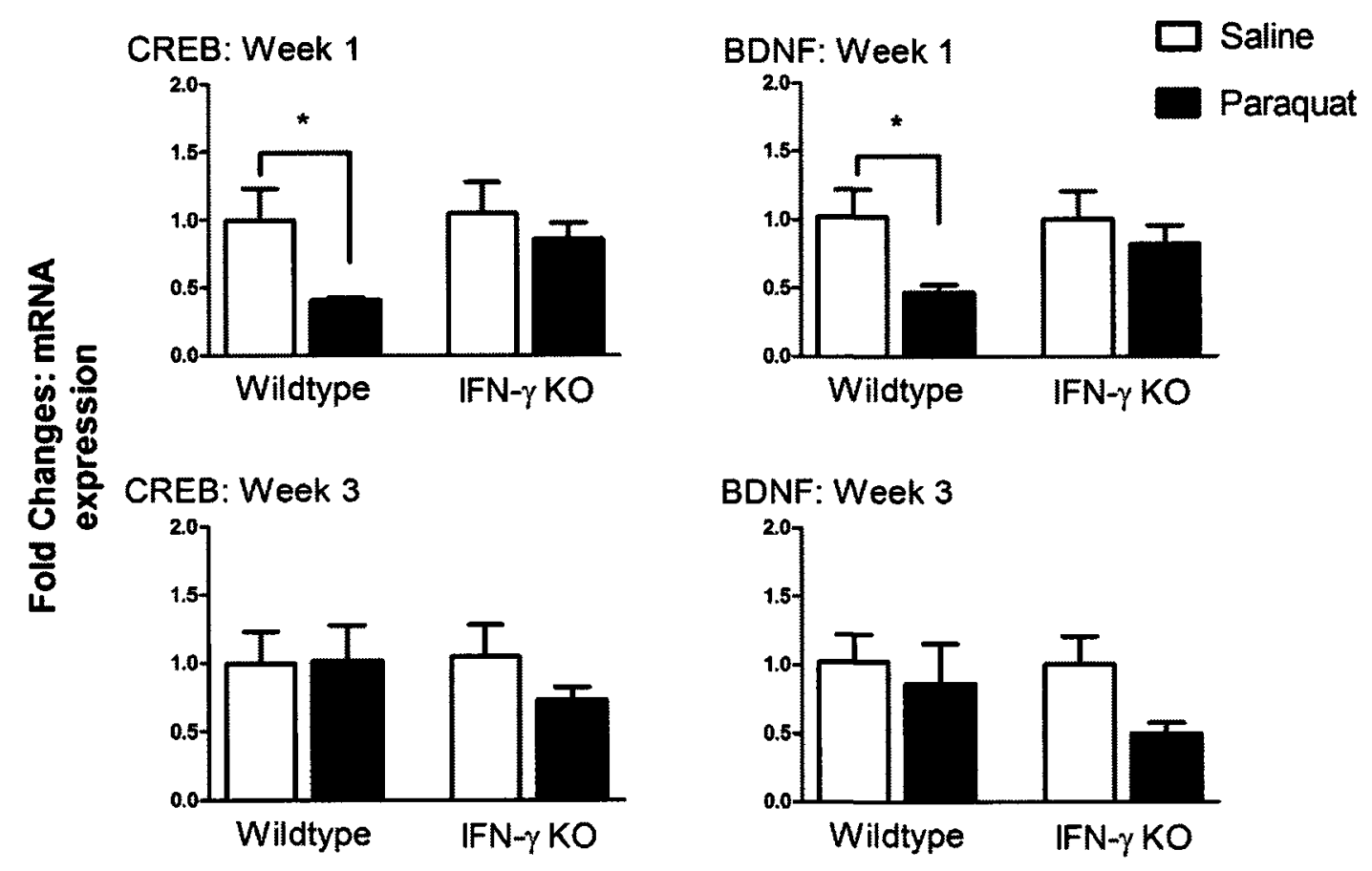

Figure 2.6 Paraquat-induced reductions of $S N C M R N A$ for $C R E B$ and BDNF in the $S N c$ are absent in IFN- $\gamma$ deficient mice.

Changes in mRNA expression for CREB and BDNF were measured by qPCR in wildtype and IFN- $\gamma \mathrm{KO}$ mice following 1 (top panels) or 3 (bottom panels) week exposure to either saline (white bars) or paraquat (black bars). Results are presented as mean fold changes \pm SEM relative to that of housekeeping genes. ${ }^{*} p<0.05,2$-way ANOVA. 
The fact that microglial activation appears to occur prior to the loss of DA neurons following toxin exposure (Kim et al., 2000; Purisai et al., 2007), and attenuation of microglial responses using anti-inflammatory agents often results in neuroprotective consequences (Du et al., 2001; Sugama et al., 2009; Radad et al., 2010), suggests a primary role for microglial-driven inflammation in PD-like pathology. Corroborating these findings, we demonstrate that the pesticide, paraquat, provoked a loss of SNc DA neurons that was associated with microglial activation and alterations of numerous oxidative, inflammatory and trophic factors. Moreover, all of these effects were prevented by IFN- $\gamma$ deficiency, indicating that the pro-inflammatory cytokine may be a primary mediator of microglial-dependent processes in PD (see Fig. 7 for conceptual model). That said, it is important to note that paraquat also reduced SNc expression of the trophic factor, BDNF, along with CREB, prior to the neurodegenerative and proinflammatory/oxidative changes. The fact that IFN- $\gamma$ deficiency also prevented this early paraquat effect underscores the importance of the cytokine not only in the evolution of inflammatory changes over time (which could conceivably be primarily linked to the neurodegenerative process or alternatively be secondarily recruited by ongoing cell loss), but also early pathological changes that could place neurons in an especially vulnerable state. 
Figure 2.7 Model of potential role of IFN-y on the pathways to pathology following paraquat exposure.

Paraquat acts as a potent inducer of oxidative distress by liberating superoxide $\left(\mathrm{O}_{2}\right.$-) and nitric oxide (NO) via NADPH oxidase and inducible nitric oxide (iNOS) enzymes on microglia. These can then combine to form the highly reactive and deleterious ONOOradical which can directly damage dopamine (DA) neurons. At the same time, the proinflammatory cytokines, tumor necrosis factor- $\alpha$ (TNF- $\alpha$ ) and interleukin-1 $\beta$ (IL-1 $\beta$ ), as well as the inflammatory enzyme, cyclooxygenase-2 (COX-2), also likely play a role in perpetuating the pro-death process in DA neurons through their impact upon oxidative and pro-apoptotic factors (e.g. the MAP kinase, JNK and BAX) and disturbances of mitochondrial functioning. The present data indicate that the cytokine, interferon- $\gamma$ (IFN$\gamma$ ), plays a crucial role in regulating microglia and these pro-death processes following paraquat exposure. It is posited that IFN- $\gamma$ acts through STAT1, NFkB and p38 to promote many of these effects. Given that paraquat reduced cyclic AMP response element binding protein (CREB) and brain derived neurotrophic factor (BDNF) expression prior to DA neuronal loss, it is possible that diminished trophic support from astrocytes rendered DA neurons vulnerable to the ongoing inflammatory and oxidative effects of paraquat. 


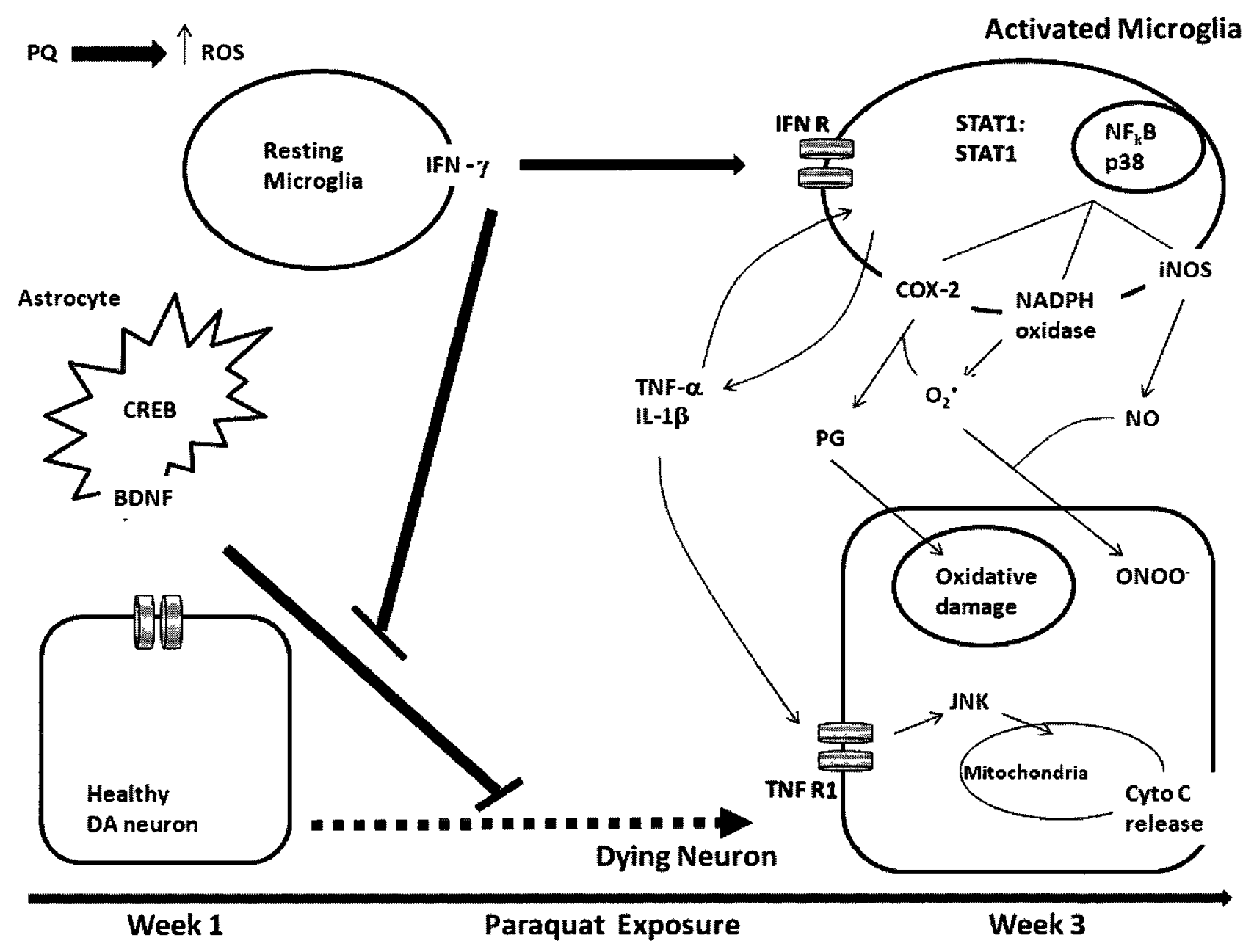


Previous reports indicate that circulating and nigrostriatal levels of IFN- $\gamma$ are increased in PD patients (Hunot et al., 1999; Ciesielska et al., 2003; Gribova et al. 2003; Mogi et al., 2007); and mice lacking IFN- $\gamma$ were resistant to the neurodegenerative effects of MPTP (Mount et al., 2007). The present study extends these findings by showing that genetic ablation of IFN- $\gamma$ prevented the loss of DA neurons associated with paraquat exposure. This is particularly important as multiple epidemiological studies have correlated pesticide exposure in general, and a few have further implicated paraquat itself, with an increased incidence of PD (Liou et al., 1997; Dhillon et al., 2008; Costello et al., 2009; Gatto et al., 2009). It is also important to note that, besides being a commonly used pesticide, paraquat provokes histopathological changes, including $\alpha$ synuclein accumulation, which more closely resemble PD than those provoked by MPTP or 6-hydroxydopamine (Manning-Bog et al., 2002; Fernagut et al., 2007; Mak et al., 2010).

Among the pro-inflammatory factors, IFN- $\gamma$ is well established as having potent stimulatory effects upon microglia, affecting the expression of surface receptors, such as MHC and TLR4, necessary for antigen presentation and responding to pathogens (Okuno et al., 2005; Kawanokuchi et al., 2006; Moran et al., 2007). Upon stimulation by IFN- $\gamma$, microglial up-regulate expression of several key enzymes involved in the generation of the oxidative burst and other inflammatory responses (Twig et al., 2005; Kawanokuchi et al., 2006). Accordingly, the present data reveal that IFN- $\gamma$ is a critical mediator of the effects of paraquat upon SNc microglial expression of NADPH oxidase (both phagocytic and non-phagocytic subunits), iNOS and COX-2. At the same time, paraquat increased SNc expression of the pro-inflammatory cytokines, IL- $1 \beta$ and TNF- $\alpha$, as well as their 
primary downstream transcription factor, NFKB and the IFN- $\gamma$ transcription factor, STAT1, which, along with NFKB, are capable of regulating iNOS and COX-2 gene expression (Paik et al., 2002; Jung et al., 2010). Since IFN- $\gamma$ deficiency abrogated virtually all of these paraquat-induced effects (as well as frank neuronal loss), these findings provide compelling evidence in support of the required nature of the cytokine for the microglial-associated oxidative and inflammatory responses underlying paraquatinduced neurodegeneration.

Paraquat-induced elevations of pro-oxidant enzymes, cytokines and key intracellular inflammatory signalling factors all paralleled the temporal pattern of SNc DA neuron loss (i.e., cell death present after 3 but not 1 week(s) of paraquat). This profile clearly suggests that a pro-inflammatory and pro-oxidative environment evolves over time following paraquat. Indeed, this slowly developing pro-death (or survival-limiting) state might be related to the time required for the inflammatory response to "ramp up" (possibly involving priming of peripheral immune cells) or conversely, might reflect the cumulative effects of paraquat, as the toxin is known to accumulate in the nigrostriatal brain regions over very long periods of time (Prasad et al., 2007, 2009). This contrasts with the more rapid microglial response that we and others have observed following intra-SNc infusion of the bacterial endotoxin, LPS (Kim et al., 2000; Ling et al., 2006; Mangano and Hayley, 2009). Yet, previous work demonstrated that when paraquat was administered at a time of heightened LPS-induced microglial response (after 2 but not 7 days), these cells maintained a morphologically active state over a month later and were associated with an enhanced neurodegenerative effect (Mangano and Hayley, 2009). Thus, the timing of the SNc neuroinflammatory response appears to depend upon the 
nature of the eliciting insult and once microglia are engaged, a prolonged response may be maintained, which might result in a sensitized response of these cells to subsequent challenges, and this could be translated into augmented damage to local DA neurons.

As already mentioned, microglial are an important source of oxidative species and mice lacking the gp91 ${ }^{\text {PHOX }}$ catalytic subunit of NADPH oxidase or those deficient in iNOS displayed significantly less DA neuronal loss following MPTP challenge (Liberatore et al., 1999; Wu et al., 2002, 2003). Similarly, IFN- $\gamma$ deficiency presently blocked the elevation of iNOS and NADPH oxidase subunits and neuronal loss provoked by paraquat. Although these enzymes are present in astrocytes and neurons, their expression is generally markedly more robust on microglia (Dringen, 2005; Sorce and Krause, 2009). Indeed, we found that $\mathrm{p} 67^{\mathrm{PHOX}}$ and gp91 ${ }^{\mathrm{PHOX}}$ were localized in microglia of wildtype mice following paraquat exposure. These findings are consistent with the limited in vitro data showing that exposure to LPS and IFN- $\gamma$ together increased NADPH oxidase and iNOS activity in glial cells (Pawate et al. 2004) and reduced DA neuron survival in midbrain cultures (Jeohn et al., 2000; Shibata et al., 2003). Given that paraquat induced the expression of iNOS and $\mathrm{gp} 91^{\mathrm{PHOX}}$, along with the other NADPH subunits within the SNc but only the $\mathrm{p} 47^{\text {PHOX }}$ subunit (which must interact with $\mathrm{p} 67^{\text {PHOX }}$ or $\mathrm{gp} 91^{\mathrm{PHOX}}$ to be effective) within the striatum, suggests that most of damaging effects of the herbicide likely occur upstream at the level of SNc DA soma. These data are consistent with reports indicating that the impact of paraquat is appreciably less on striatal DA terminals, compared to SNc soma (McCormack et al., 2002; Ciccetti et al, 2005). 
Although downstream oxidative or inflammatory effects of IFN- $\gamma$ would presumably stem from gene regulation through its main transcription factor, STAT1 (Kakar et al. 2005), the cytokine can also act through STAT-independent posttranslational mechanisms and, like IL-1 $\beta$ and TNF- $\alpha$, can affect parallel MAP kinase pathways (Chesler et al., 2004). Importantly, these factors are fundamentally linked to oxidative processes; indeed, JNK or p38 antagonists blocked oxidative radical production by interfering with the translocation of NADPH oxidase subunits (Yamamori et al., 2002; Dang et al., 2003; Brown et al., 2004; Niwa et al., 2004; Kuan et al., 2005; Peng et al., 2004, 2007). Hence, the fact that STAT1 expression was modulated by paraquat and IFN$\gamma$ deficiency in a manner identical to JNK and p38 once again supports an integral role for IFN- $\gamma$ in a complex multi-pathway regulation of DA neuronal survival (see Figure 7).

Besides oxidative and inflammatory factors, PD has been associated with alterations of trophic factors, such as GDNF and BDNF (Howells et al., 2000; Chauhan et al., 2001; Masaki et al., 2003; Guerini et al., 2009). Indeed, virtually all animal models of PD have demonstrated some degree of neuroprotection following GDNF administration (Eslamboli et al., 2005; Zheng et al., 2005; Huang et al., 2010). Similarly, BDNF has well documented pro-survival effects and is found in within the DA neurons of the SNc (Seroogy et al., 1994; Zhang et al., 2007). Although GDNF expression was below detection in the present study, we did find that paraquat reduced BDNF and its upstream regulator, $\mathrm{CREB}$, within the $\mathrm{SNc}$; and once again, IFN- $\gamma$ deficiency prevented this effect. Interestingly, $\mathrm{BDNF}$ and $\mathrm{CREB}$ reductions occurred at week 1 of the paraquat regimen, in contrast to the later occurring elevations of the aforementioned oxidative and inflammatory factors. In effect, the diminished trophic support presumed with the BDNF 
and CREB reductions early in the regimen could engender a vulnerability to the oxidative and inflammatory stressors that were later promoted by paraquat administration.

In short, the present findings support an integral role of IFN- $\gamma$ in mediating multiple interconnected pathways leading to neurodegeneration following paraquat exposure (Figure 7). It is our contention that paraquat enhanced the vulnerability of SNc DA neurons by dampening BDNF support prior to a loss of DA neurons and IFN- $\gamma$ deficiency prevented this early reduction in trophic support. Subsequent exposure to paraquat-induced production of free radicals promoted the release of microglialdependent pro-oxidant and pro-inflammatory factors. Chronic exposure to this pesticide can cause these microglial cells to assume a chronically hyperactive state characterized by the excessive production of oxidative and inflammatory factors. These changes, together with reductions of trophic factors (BDNF and CREB) might favour a state of diminished resiliency and plasticity of SNc neurons. We further posit that IFN- $\gamma$ is the critical inflammatory mediator that shifts microglia into a prolonged state of activation and release of toxic factors, eventually resulting in the death of DA neurons. These findings further the scope of inflammatory processes linked to PD to include IFN- $\gamma$ as a potentially important therapeutic target. 
Proinflammatory cytokines and Parkinson's disease, 88

\section{Chapter 3}

Inflammatory priming of the substantia nigra influences the impact of later paraquat exposure: Neuroimmune sensitization of neurodegeneration 


\begin{abstract}
Activation of microglia along with the release of inflammatory cytokines and oxidative factors often accompanies toxin-induced degeneration of substantia nigra pars compacta (SNc) dopamine (DA) neurons. Multiple toxin exposure may synergistically influence microglial-dependent DA neuronal loss and, in fact, pre-treatment with one toxin may sensitize DA neurons to the impact of subsequent insults. Thus, we assessed whether priming SNc neurons with the inflammatory agent, lipopolysaccharide (LPS), influenced the impact of later exposure to the pesticide, paraquat, which has been reported to provoke DA loss. Indeed, LPS infusion into the SNc sensitized DA neurons to the neurodegenerative effects of a series of paraquat injections commencing 2 days later. In contrast, LPS pre-treatment actually protected against some of neurodegenerative effects of paraquat when the pesticide was administered 7 days after the endotoxin. These sensitization and de-sensitization effects were associated with altered expression of reactive microglia expressing inducible immunoproteasome subunits, as well as variations of fibroblast growth factor and a time-dependent infiltration of peripheral immune cells. Circulating levels of the inflammatory cytokines, interleukin (IL)-6, IL-2, tumor necrosis factor- $\alpha$ and interferon- $\gamma$ were also time-dependently elevated following intra-SNc LPS infusion. These data suggest that inflammatory priming may influence DA neuronal sensitivity to subsequent environmental toxins by modulating the state of glia land immune factors, and these findings may be important for neurodegenerative conditions, such as Parkinson's disease (PD).
\end{abstract}




\section{Introduction}

Parkinson's disease (PD) stems from degeneration of the dopamine (DA) neurons of the substantia nigra pars compacta( $\mathrm{SNc})$, resulting in reduced $\mathrm{DA}$ release from terminals of the striatum (Blandini et al., 2000; Hirsch et al., 1988; Langston, 2006). Although a few rare familial forms of PD exist (Dawson and Dawson, 2003; Smith et al., 2005), most cases are idiopathic and probably arise from exposure to a constellation of environmental factors, probably in conjunction with a genetic vulnerability (Betarbet et al., 2006a; Betarbet et al., 2000; Kelada et al., 2006). In this regard, epidemiological and animal studies have linked PD with exposure to several commonly used pesticides, such as paraquat and rotenone (Andersen, 2003a; Cicchetti et al., 2005; Thiruchelvam et al., 2000b; Wu et al., 2003). These pesticides provoke a loss of DA neurons, alterations of DA metabolism, and induce the formation of $\alpha$-synuclein containing Lewy bodies in rodents (Betarbet et al., 2006a; Sherer et al., 2007). Moreover, exposure to multiple toxins, such as paraquat and the fungicide, maneb, may synergistically increase the risk of PD (Andersen, 2003a; Cicchetti et al., 2005; Thiruchelvam et al., 2000a).

Neuroinflammatory processes may be one major mechanism through which environmental toxins contribute to neurodegeneration in PD (Carvey et al., 2003; Dickman, 2001; Koustilieri et al., 2002). Indeed, post-mortem PD brain tissue and common animal models of the disease (exposure to MPTP or 6-OHDA) activated microglia coupled with increased expression of oxidative factors, as well as proinflammatory cytokines, including tumor necrosis factor- $\alpha$ (TNF- $\alpha)$ and interleukin- $1 \beta$ (IL-1ß) (Depino et al., 2003; Mandel et al., 2000a; Mogi et al., 1996; Mogi et al., 1994; Nagatsu et al., 2000; Nagatsu and Sawada, 2005). Similarly, paraquat, rotenone and the gram-negative bacterial endotoxin, lipopolysaccharide (LPS), stimulate microglial release 
of superoxide free radicals (Kim et al., 2000; Le et al., 2001; Qian et al., 2006; Qin et al., 2004) and co-administration of LPS with either pesticide synergistically augmented DA loss through a microglial-dependent mechanism (Gao et al., 2002a; Gao et al., 2003a; Gao et al., 2003c; Liu et al., 2003; Purisai et al., 2007). In addition to glial variations, alterations of interferon- $\gamma(\mathrm{IFN}-\gamma)$ producing T lymphocytes were reported in PD patients and following MPTP treatment (Baba et al., 2005; Czlonkowska et al., 2002; KurkowskaJastrzebska et al., 1999b). Peripheral immune cells could influence neurodegeneration by affecting microglial activity or the production of neurotrophic growth factors, such as glial derived neurotrophic factor (GDNF) or fibroblast growth factor (FGF) (Benner et al., 2004; Laurie et al., 2007; Le et al., 2001; Walker et al., 1998).

Other elements of the immune system, including the immunoproteasome, which are involved in antigen processing, might also be activated by environmental toxins. Exposure to immunogenic antigens (e.g. LPS) normally induces the catalytic subunits, low molecular mass polypeptide (LMP)-2 and LMP-7, which replace constitutive proteasome subunits (Marques et al., 2004), resulting in the formation of a functional immunoproteasome (Groettrup et al., 2001). Although substantial evidence indicates that PD patients have a dysfunctional 20S proteasome (Doherty et al., 2002; McNaught et al., 2002; Nakamura et al., 2006), little is known regarding immunoproteasome functioning in PD. However, altered expression of the LMP-2 and LMP-7 subunits were reported within the cortex and striatum of Huntington's and Alzheimer's disease patients (DiazHernadez et al., 2003; Mishto et al., 2006) and impaired motor performance was found in LMP-2 deficient mice (Martin et al., 2004). As already mentioned, LPS exposure may synergistically enhance neuroinflammatory and neurodegenerative processes when co- 
administered with a DA toxin (Gao et al., 2002a; Gao et al., 2003a; Gao et al., 2002b; Gao et al., 2003b). Moreover, LPS pre-treatment (priming) enhanced the behavioral and neurochemical effects of a cytokine or neurogenic stressor, when these challenges were given some time later (cross-sensitization) (Hayley et al., 1999; Hayley et al., 2001; Schmidt et al., 1995). Hence, it was hypothesized that intra-SNc infusion of LPS would sensitize inflammatory processes, enhancing the neurodegenerative effects of later exposure to the DA toxin, paraquat (cross-sensitization). Furthermore, as sensitization effects related to inflammatory processes may vary over time (Cunningham et al., 2005; Hayley and Anisman, 2005), it was of particular interest to establish whether such effects were dependent upon the timing between exposure to the endotoxin and the pesticide. Indeed, previous studies using animal models of stroke, indicated that immune challenges, such as LPS or TNF- $\alpha$, may sensitize or de-sensitize (protect) neurons to the impact of ischemia, depending upon the timing of exposure to the two challenges (Hayley and Anisman, 2005; Rosenzweig et al., 2004). Finally, we assessed whether changes in inflammatory immune factors (LMPs, cytokines), the growth factor, FGF, and activation of microglia or infiltrating peripheral immune cells were associated with any individual or sensitizing neurodegenerative effects of the LPS and paraquat treatments.

\section{Materials and methods}

Single housed, male, C57BL/6 mice obtained from Charles River Laboratories (Laprairie, Quebec, Canada) at 9-10 weeks of age were acclimated to the vivarium for 2 weeks prior to experimental procedures being initiated. Mice were maintained on a $12-\mathrm{h}$ light/12-h dark cycle in a temperature $\left(21^{\circ} \mathrm{C}\right)$ controlled room with an ad libitium diet of Ralston Purina mouse chow and water. 


\section{Overall experimental rationale}

Two experiments were conducted to determine, firstly, the temporal profile of cytokine and inflammatory cellular changes provoked by direct infusion of relatively low doses of LPS into the SNc, and secondly, whether administration of paraquat at certain intervals following the endotoxin resulted in augmented or diminished neurodegenerative responses associated with distinct inflammatory changes.

\section{Surgery and LPS infusion}

All mice (10-12 weeks of age) underwent stereotaxic surgery wherein indwelling cannulae (26-gauge stainless steel) were implanted above the SNc (bregma anteroposterior $-3.16 \mathrm{~mm}$; lateral $\pm 1.2 \mathrm{~mm}$; ventral $-4.0 \mathrm{~mm}$ ) and given a 6-day convalescence period prior to commencement of experimentation. Thereafter, an infusion of either saline or LPS $(0.1$ or $3.0 \mu \mathrm{g})$ was given in $2 \mu \mathrm{l}$ of fluid delivered into the SNc over a 5 minutes period from polyethylene tubing connected to a Hamilton micro-liter syringe driven by a Harvard Apparatus Pico Plus syringe pump. In contrast to studies involving restraint of rodents during central infusions, mice in the present study were able to freely move about in their home cages, thereby minimizing the stress associated with this procedure.

\section{Experiment 1: intra-SNc LPS time course}

At either 90 minute, 2- or 7-days following intra-SNc infusion of saline or LPS, half of the mice ( $n=8-10$ /group) were rapidly decapitated and the $\mathrm{SNc}$ and striatal nuclei collected by micro-punch and the remaining half underwent transcardial perfusion. Specifically, using a biopsy needle $(1.0 \mathrm{~mm}$ diameter) tissue was micro-punched from coronal sections $(0.5 \mathrm{~mm}$ ) of the SNc (bregma -2.92 to -3.88 ) and striatal (bregma 0.38 to 
1.10) nuclei (Hayley et al., 2004a; Hayley et al., 2001), following the Atlas of Franklin and Paxinos (1997).

Brain punches were then stored at $-80^{\circ} \mathrm{C}$ until they were ready to be processed for cytokine protein concentrations using a Luminex multiplex laser-based bead assay. Plasma was also extracted from trunk blood for cytokine determinations. Briefly, blood was collected in EDTA treated tubes and then centrifuged for $10 \mathrm{~min}$ at $3600 \mathrm{rpm}$ and supernatant removed. The remaining mice were perfused with $10 \mathrm{ml}$ of cold saline followed by $4 \%$ PFA; brains were removed and post-fixed for an additional 24-hr. Brains were then cryo-protected in a $20 \%$ sucrose solution with $0.1 \mathrm{M}$ PBS and $0.02 \%$ sodium azide (pH 7.4) for at least 3 days before immunohistochemical assessment of glial and immune cell activation.

\section{Experiment 2: LPS-paraquat sensitization}

Two or seven days following intra-SNc infusion of saline or a low dose of LPS $(0.1 \mu \mathrm{g} / 2 \mu \mathrm{l})$, mice $(n=10 /$ group $)$ were administered either saline or paraquat $(10 \mathrm{mg} / \mathrm{kg}$; i.p.) three times a week for a 3 week period. Mice were then sacrificed 5 days following their last paraquat or saline injection and perfused (as described for Experiment 1) for immunohistochemical analyses of glia, FGF and quantification of DA neuronal loss by TH and FluoroJade B immunohistochemistry.

\section{Multiplex Luminex procedure}

The Luminex 100 is a suspension based bead array system that can detect up to 100 different analytes in a single $50 \mu$ sample. Sets of microspheres ( $5.6 \mu \mathrm{m}$ beads) are internally dyed with different ratios of fluorophores, each conjugated to a different capture probe (cytokine specific antibody). Following incubation, a classification laser 
identifies the particular cytokine bound and a second reporter laser quantifies the signal present. We utilized a custom multiple cytokine detection kit (Beadlyte Mouse MultiCytokine Detection System, Upstate Cell Signalling Solutions) to detect circulating and brain levels of IL-2, IL-6, IFN- $\gamma$ and TNF- $\alpha$ at three different times following LPS infusion (Experiment 1). In recent studies using this procedure, we have reliably observed distinct patterns of elevations of these cytokines in response to several insults, ranging from middle cerebral artery occlusion to chronic psychological stressors.

To determine cytokine concentrations, brain punches were homogenized and assayed for protein while plasma was treated with a serum diluent (Upstate Cell Signalling, Cat\#43-007); apart from these distinctions all samples underwent the exact same process. Following three brief washes, $60 \mu \mathrm{l}$ of lysis buffer was added to the samples and they were immediately homogenized, centrifuged at $6000 \mathrm{rpm}$ for $10 \mathrm{~min}$ at $4^{\circ} \mathrm{C}$ and $50 \mu \mathrm{l}$ of the supernatant was collected for use in the Luminex analysis. To prepare standards for Luminex analysis, $5000 \mathrm{pg}$ of Multi-Cytokine 2 standard was resuspended in $1 \mathrm{ml}$ serum diluent and vortexed at a medium speed for $15 \mathrm{~s}$, following which serial dilutions were prepared. After $25 \mu \mathrm{l}$ of Beadlyte Cytokine Assay Buffer was added to the wells, plates were vortexed and a vacuum manifold applied to remove excess liquid. For serum samples, $25 \mu \mathrm{l}$ of serum diluent and $25 \mu 1$ of sample were added to each well. Following $20 \mathrm{~min}$ incubation on a shaker, the anti-mouse multi-cytokine beads were vortexed, sonicated and $25 \mu 1$ of the bead solution added to the wells. Following a brief vortex, plates were then incubated overnight at $4^{\circ} \mathrm{C}$. Thereafter, samples were re-suspended in $50 \mu \mathrm{l}$ of Beadlyte Cytokine Assay Buffer and the vortex and washing procedures repeated. Finally, $25 \mu 1$ of biotin conjugated cytokine beads were 
added for 90 min incubation in the dark. Just prior to the end of the incubation period, the Beadlyte Streptavidin-PE was diluted (1:25) and $25 \mu 1$ was added to each well for the final 30 min incubation. The assay was then halted using $25 \mu 1$ of Beadlyte Stop Solution. Filter plates were then read in a Luminex 100 instrument, fitted with a five-parameter logistic regression curve using QT Masterplex software (MiraiBio, Hitachi, CA) (Hulse et al., 2004).

\section{Immunohistochemistry}

Brains were cut using a Shandon AS620 Cryotome (Fisher Scientific, Ottawa, ON) and coronal sections (14 $\mu \mathrm{m}$ thick) were obtained from every $6^{\text {th }}$ section of the striatum and every $4^{\text {th }}$ section of the SNc between the aforementioned bregma levels. Sections were then incubated with primary antibodies (0.01M PBS, $0.3 \%$ Triton X-100) overnight at $4^{\circ} \mathrm{C}$ and following two washes with $0.01 \mathrm{M}$ PBS, were incubated with secondary antiserum for 2 -hr at room temperature. Thereafter, slides were cover-slipped with anti-fade and stored at $-20^{\circ} \mathrm{C}$.

Experiment 1, glial activation within the SNc was evaluated using primary antibodies specific for microglia (rat anti-mouse CD11b; 1:1000, Serotec) and astrocytes (rabbit anti-mouse GFAP, 1:500, Dako). To assess the possibility that LPS may have influenced the infiltration of peripheral immune cells around the meninges adjacent to the SNc, CD3immunoreactivity (rat anti-mouse 1:400, Serotec) was used as a marker for Tlymphocytes and $\mathrm{CD} 11 \mathrm{~b}$ as a marker for peripheral macrophages.

As well, to determine the impact of LPS upon central immunoproteasome activity, antiLMP-2 (rabbit anti-mouse 1:400, Serotec) and anti-LMP 7 (rabbit anti-mouse 1:400, Serotec) immunostaining was conducted across multiple levels of the SNc. Donkey anti- 
rat and anti-rabbit CY3 conjugated secondary anti-sera (1:200, Jackson Laboratories) was used for visualization of these glial and immune cell markers. In cases of double labelling, anti-LMP and anti-CD11b or anti-mouse TH were co-incubated, as already described, and visualized using anti-rabbit CY3 (1:200, Jackson) and anti-rat or antimouse FITC (1:100, Jackson).

Microglial reactivity was semi-quantitatively evaluated by two blind raters (>90\% inter-rater reliability) that assessed the degree of morphological change using a 0-3 rating scale, with a rating of 0 referring to resting glial state (highly ramified with thin processes), 1 reflecting an intermediate reactive state in which less than 10 cells/SNc could be considered moderately activated (thickened, short processes with a compact soma), a rating of 2 was given when the majority of cells were intermediately activated with occasional highly activated cells (these essentially have an amoeboid, macrophagelike appearance, being spherical in shape and lacking processes) and a rating of 3 was given when a large number of cells displayed the most highly activated amoeboid shape.

Survival of DA neurons in Experiment 2 was assessed by counting the number of $\mathrm{TH}^{+}(1: 1000$, ImmunoStar) neurons present through the rostral-caudal axis of the SNc and making between group comparisons at specific bregma levels. Briefly, at least 2 sections were obtained from each animal representing each of five levels from -3.08 to 3.40 relative to bregma. Sections from each level/animal were averaged for the number of $\mathrm{TH}^{+}$neurons with a total of $8-10$ animals/group analyzed. As previously described (Hayley et al., 2004a), density of the $\mathrm{TH}^{+}$terminals within the striatum was quantified using a software program available for free download from the University of Texas, UTHSCSA (Image-Tool, Version 3.0). In all cases, analyses were performed by an 
individual unaware of the experimental treatments and each tissue sample was quantified relative to its own non-stained background and then compared to the other treatment groups.

Additionally, FluoroJade B, immunohistochemistry was used to assess whether ongoing neurodegeneration may have been occurring in any survival neurons following the treatments. Briefly, slides were incubated with $0.0001 \%$ FluoroJade B (Vector) for 30 min after sequential immersion in $1 \% \mathrm{NAOH}$, alcohol $(80 \%$ followed by $70 \%)$ and $0.06 \%$ potassium permanganate.

Finally, some sections were double labelled with mouse anti-TH (1:800, ImmunoStar) and the mature neuronal marker, rabbit anti-NeuN (1:800, Chemicon) and visualized using anti-TH Texas Red (1:150, Vector) and anti-rabbit FITC (1:100, Vector). The number of surviving $\mathrm{TH}^{+}$and $\mathrm{TH}^{-}$neurons $\left(\mathrm{NeuN}^{+}\right)$were then quantified using a Nikon C1 Confocal system. Thus, we assessed whether the LPS-paraquat treatment selectively affected DA neurons $\left(\mathrm{TH}^{+} / \mathrm{NeuN}^{+}\right)$or induced a global loss of these plus nonDA neurons $\left(\mathrm{TH}^{-} / \mathrm{NeuN}^{+}\right)$within the vicinity of the SNc. As described for Experiment 1, microglial (CD11b) and astrocyte (GFAP) immunohistochemistry was performed on SNc and striatal sections. As well, FGF-2 immunoreactivity within the striatum was determined using the primary antibody (rabbit anti-mouse 1:100, Santa Cruz), together with biotinylated goat anti-rabbit anti-serum (1:100, Vector) and visualized with streptavidin conjugated Texas Red (1:50, Vector).

\section{Western blot}

Western blot analyses of tissue punches from the striatum were conducted to assess changes in astrocyte expression that may be associated with the LPS and paraquat elicited 
loss of $\mathrm{TH}^{+}$terminals. Specifically, the primary antibody GFAP (1:5000, Dako) was used and $\beta$-actin (1:20,000, Sigma) applied as a loading control. Following our previous procedures (Hayley et al., 2004a), striatal tissue punches were diluted in an extraction buffer $(5.0 \mathrm{M} \mathrm{NaCl}, 0.5 \mathrm{M}$ EDTA,100mM EGTA, 1M DTT, 50\% glycerol, $0.1 \mathrm{M}$ PMSF, $10 \mathrm{mg} / \mathrm{ml}$ leupeptin, $5 \mu \mathrm{g} / \mathrm{ml}$ aprotinin, $1 \mathrm{M}$ glycerol-phosphate, $0.5 \mathrm{M} \mathrm{NaF}$, and $100 \mathrm{mM}$ Na-orthovanadate), sonicated, centrifuged at $14,000 \mathrm{rpm},(15 \mathrm{~min})$ and supernatant assessed for protein concentration using a BioRad protein assay. Thereafter, $30 \mu \mathrm{g}$ of protein was analyzed by SDS/PAGE followed by western blot.

\section{Behavioural analyses}

All behavioural testing was conducted between $08: 00$ and 11:00 $\mathrm{h}$ to minimize influences of circadian variations. One hour following the final paraquat/saline injections mice were assessed for PD-like symptoms as reflected by three qualitative indices of motor impairment; posture, gait and muscle tone. Specifically, mice were placed in an open area $(30 \mathrm{~cm} \times 30 \mathrm{~cm} \times 30 \mathrm{~cm})$ for $3 \mathrm{~min}$, during which behavioural symptoms were scored on a 3-point rating scale by two independent blind raters.

Individual ratings of these three symptoms were then compiled to create an average, thought to reflect the overall motor impairment of the animal. Individual ratings of posture were determined by assessing the hunched/arched appearance of the spine of mice (a rating of 0 was assigned for normal posture, a 1 was assigned if an arched back appearance became apparent within 3 minute of being placed in an open field, a rating of 2 was given to mice that immediately displayed a hunched appearance that lasted for the entire 3 minute in the arena). In terms of gait, mice were evaluated on the style of stepby-step motion utilized in the arena. If the paw-placement appeared normal with a well 
coordinated gait a rating of 0 was assigned, whereas if the mouse displayed either dragging the hind-paws in an uncoordinated manner or shuffling of the hind limbs producing a "start-stop" movement, a rating of 1 was assigned. If both abnormal behaviours were concomitantly present a rating of 2 was given. For muscle tone, mice were briefly (10 seconds) suspended in the vertical position and hind-legs were scrutinized for limb placement and struggling behaviour. Mice with a robust struggle reflex in the vertical position and no signs of splayed limbs were given a normal rating of 0 . Animals showing mildly splayed hind-paws with the absence of normal struggling in the vertical position were assigned a rating of 1 . Those that failed to show any sign of struggle in the vertical position and had severe splayed hind-paws were given a rating of 2.

\section{Statistical analyses}

Data were analyzed by ANOVA, followed by Fisher's planned comparisons $(p<$ 0.05) where appropriate. Data were evaluated using a StatView (Version 5) statistical software package available from the SAS Institute, Inc.

\section{Results}

Experiment 1: LPS induced time-dependent SNc microglial activation

Both concentrations of LPS provoked a similar degree of activation of microglia, hence, for the sake of clarity; photomicrographs are only presented for the lower dose of the endotoxin $(0.1 \mu \mathrm{g})$. As shown in Figure 3.1, intra-SNc LPS infusion provoked a robust increase of SNc CD1 $\mathrm{bb}^{+}$immunoreactivity, peaking at the 2-day interval on the ipsilateral injection side. In contrast, $\mathrm{CD} 11 \mathrm{~b}^{+}$immunostaining within the contra-lateral noninjected SNc was not affected by the LPS treatments (data not shown). At 2-days following LPS infusion, $\mathrm{CD}_{1} 1 \mathrm{~b}^{+}$microglia within the SNc, below the site of injection, 
displayed morphological characteristics indicative of a highly activated state, including shortened thick dendritic processes and compact rounded soma. By contrast, saline treated mice displayed thin, highly ramified branching patterns and fine, less obvious soma characteristic of a microglial resting state.

Semi-quantitative ratings confirmed that CD11b hyper-reactive microglial cells were maximally activated 2-days after the endotoxin. At the 7-day interval, there were some intermediately reactive cells, along with those that appeared to be in a resting state, see Figure 3.1. The fact that CD11b was still modestly elevated 7-days following LPS treatment, suggests that a single exposure to the endotoxin had protracted effects upon SNc microglial reactivity. Paralleling the changes in $\mathrm{CD}_{11} \mathrm{~b}^{+}$immunostaining, a robust elevation of LMP-2 and -7 immunoproteasome subunit staining was observed 2-days after the endotoxin (Figure 3.1); moreover, these still remained elevated after 7-days, particularly LMP-7. Within the SNc, both LMPs were largely co-localized with CD11 $\mathrm{b}^{+}$ cells, thereby indicating the expression of these immunoproteasome subunits on microglia. However, there was no evidence of either of the LMPs being expressed on $\mathrm{TH}^{+}$neurons within the SNc (data not shown).

Insert Figure 3.1 about here

Infiltration of peripheral immune cells: CD3, CD11b and LMP 2 and 7 immunoreactivity at the meninges

As shown in Figure 3.2, intra-SNc LPS infusion augmented CD3 immunoreactivity around the meninges just ventral to the SNc. Although there was only a modest increase in the CD3 staining around the meninges after 2-days, a dramatic 
Figure 3.1 Intra-nigral LPS induced time-dependent changes in SNc microglia immunoproteasome expression.

As indicated by the photomicrographs, microglial cells of saline treated mice $\left(\mathrm{CD} 11 \mathrm{~b}^{+}\right.$ staining) were thin and highly ramified, characteristic of a resting state. Exposure to LPS $(0.1 \mu \mathrm{g})$ moderately influenced microglia activation after $90 \mathrm{~min}$; however, 2-days following LPS induced profound morphological changes, wherein microglial cells displayed shortened thick dendritic processes and compact rounded soma. Microglia cells remained modestly elevated at 7-days following LPS infusion. As depicted in the table, a semi-quantitative rating scale confirmed that a single exposure to LPS caused a peak in ratings of microglial reactivity 2-days following LPS infusion. Paralleling the CD11 $\mathrm{b}^{+}$ immunostaining, variations in the immunoproteasome subunits LMP-2 and LMP-7 were induced by a single LPS infusion. Specifically, saline treated mice displayed low levels of LMP-2 and LMP-7 immunoreactivity within the SNc; however, both LMP-2 and LMP-7 were greatly elevated 2-days following LPS. The photomicrographs shown on the bottom right (see arrows) reveal that a 2-day delay between LPS+paraquat treatments induced CD11b immunoreactivity that was predominately co-localized with LMP-2 (the same was true for LMP-7, not shown). 


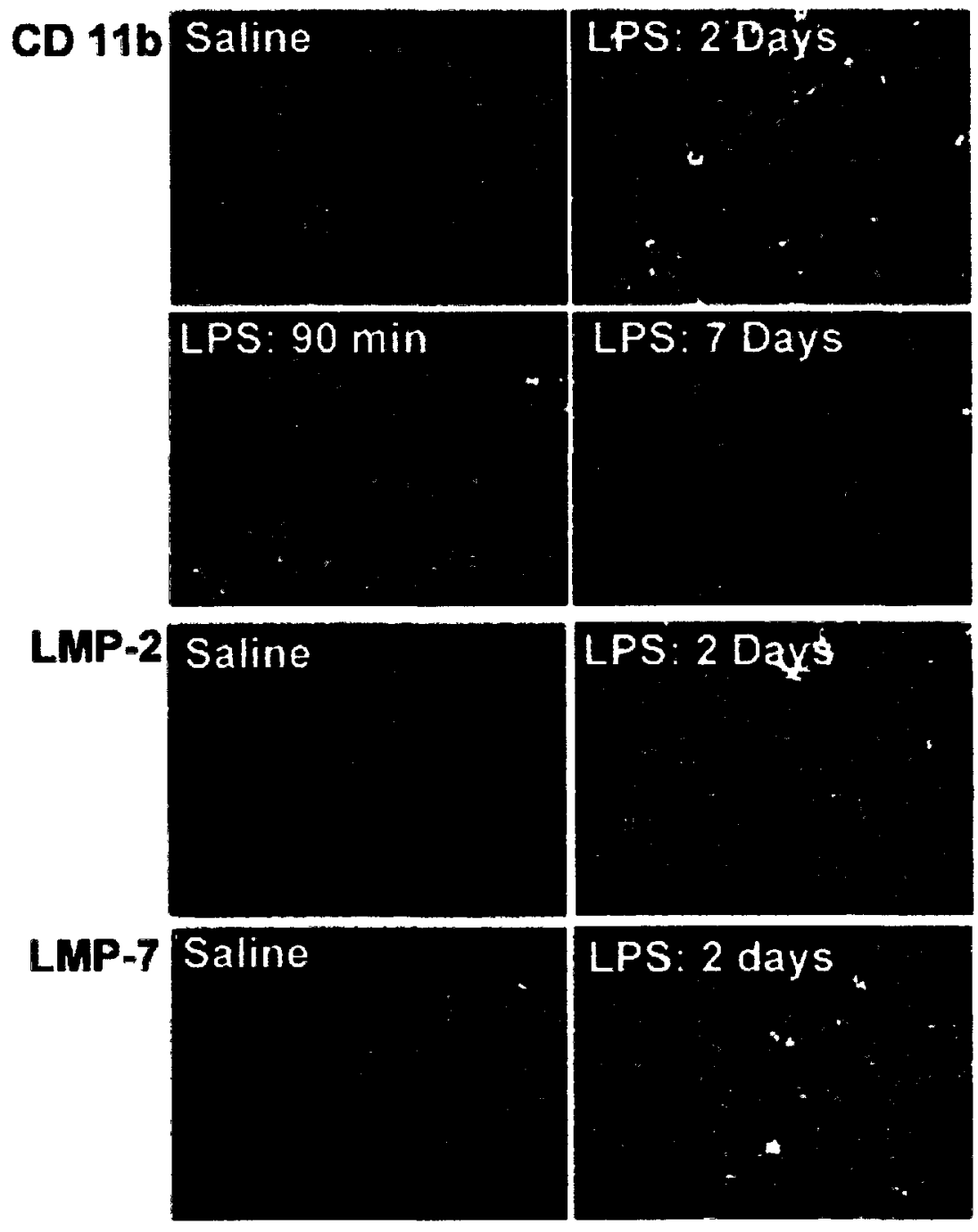

\begin{tabular}{ll}
\hline Treatment & $\begin{array}{l}\text { Microglia Rating } \\
\text { (Mean } \pm \text { SEM) }\end{array}$ \\
\hline Sellne & $0.0 \pm 0.0$ \\
90 minutes & $1.0 \pm 1.0$ \\
Day 2 & $2.7 \pm 0.3$ \\
Day 7 & $0.4 \pm 0.3$ \\
\hline
\end{tabular}

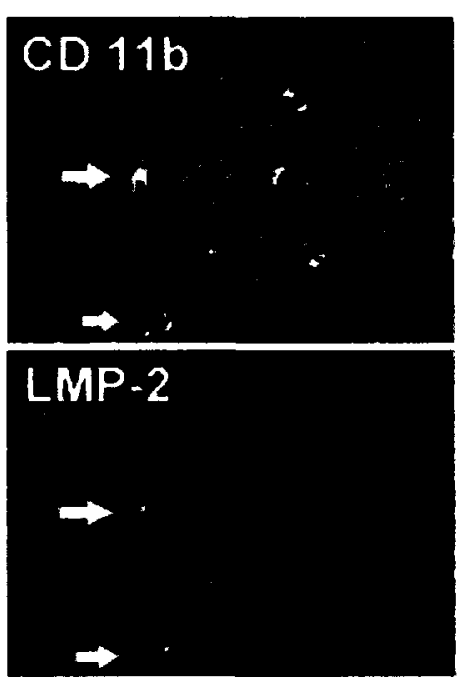


elevation in the number of cells expressing the CD3 T-lymphocyte marker was evident 7days following the endotoxin (Figure 3.2). These cells were small, ovoid and consistent with the appearance of T-lymphocytes that were previously shown to infiltrate the brain parenchyma following TNF- $\alpha$ administration (Farkas et al., 2003). Paralleling these CD3 changes, LMP-2 and LMP-7 labelling of meningeal cells located ventral to the SNc displayed an identical temporal pattern of labelling (Figure 3.2). In contrast, CD11b labelling of cells lining the meninges (which likely reflect macrophages) was markedly elevated above control levels at both the 2- and 7-day intervals following LPS infusion (Figure 3.2).

Finally, a pattern of CD11b, CD3and LMP labelling that was identical to that observed at the meninges, was also apparent around the third ventricle (data not shown), suggesting that these immune cells were present throughout the meningeal and ventricular lining of the brain.

Insert Figure 3.2 about here

Central and peripheral cytokine alterations following LPS

Infusion of LPS into the SNc provoked marked changes of several cytokines within the SNc and striatum, as well as in peripheral circulation. In particular, two prominent patterns of cytokine alterations emerged, an early cytokine elevation that peaked after $90 \mathrm{~min}$ and a late occurring pattern, wherein levels were increased 7-days following the endotoxin. 


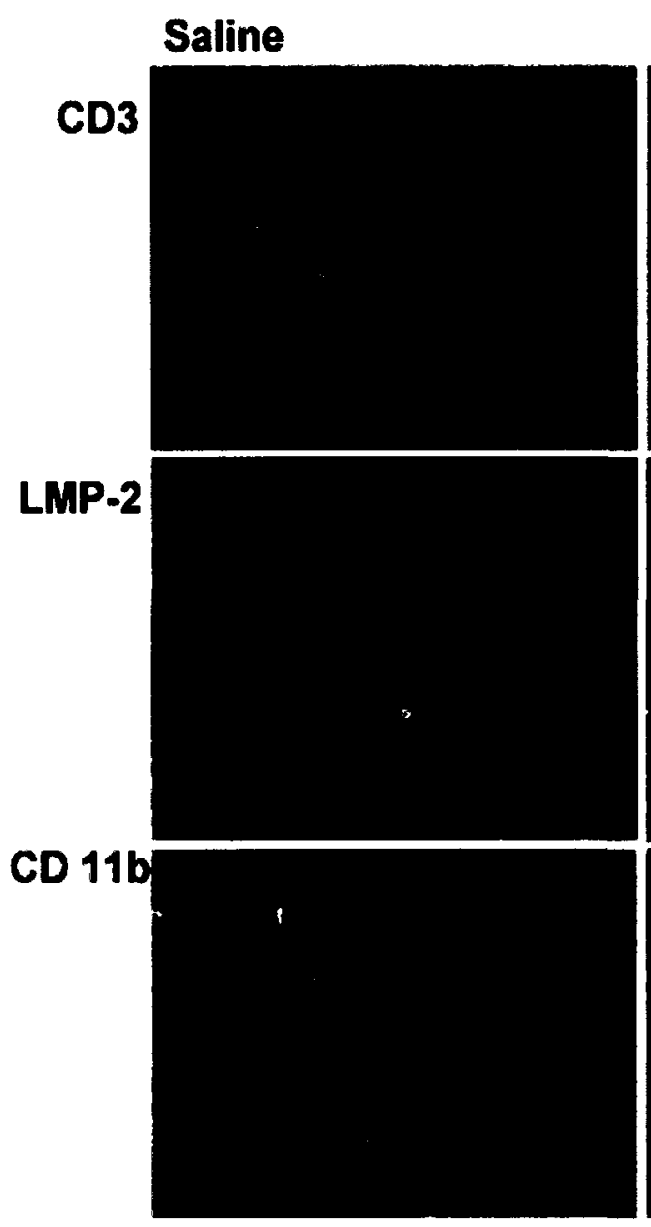

LPS: 2 Days
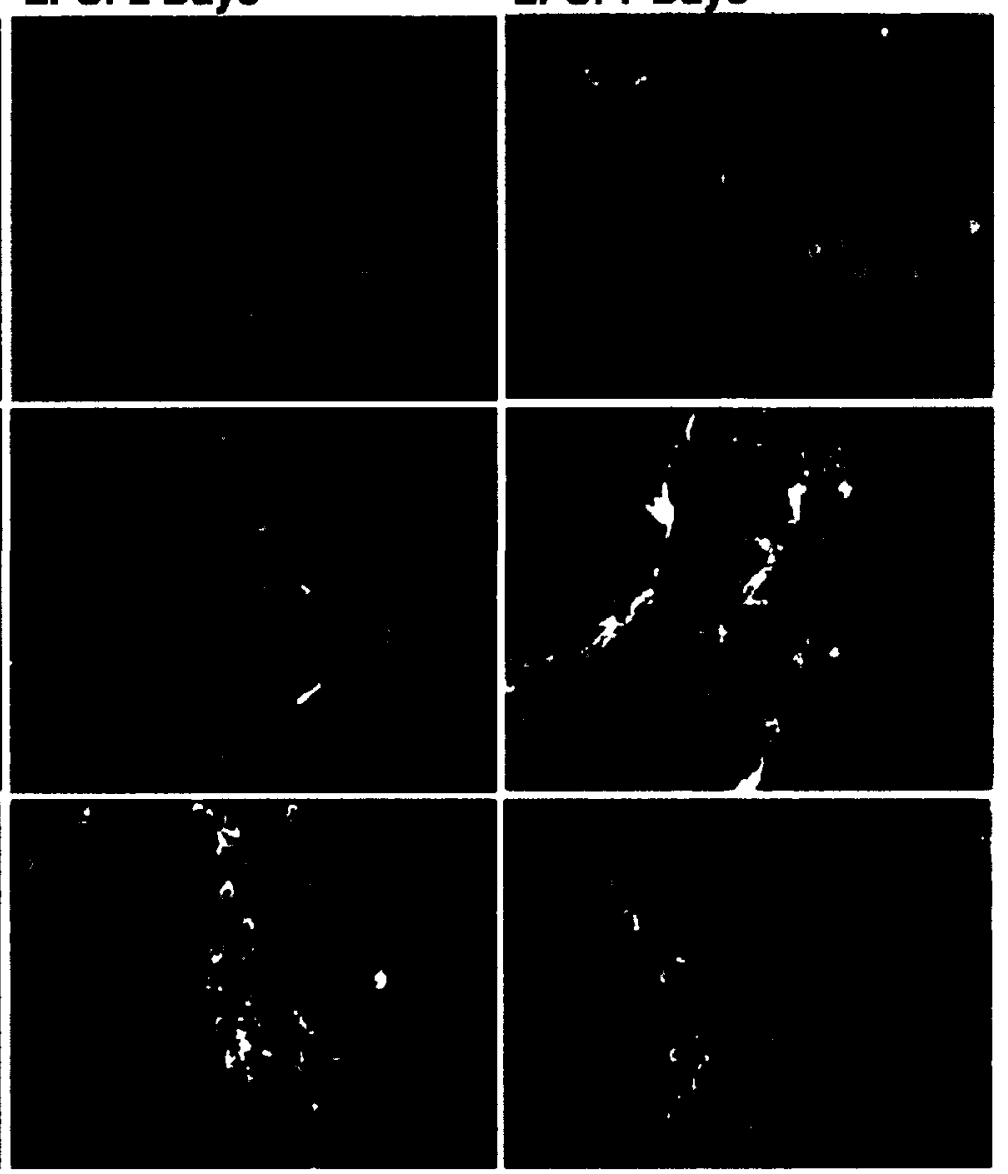

LPS: 7 Days

Figure 3.2 Intra-nigral infusion of LPS provoked time-dependent elevation of CD3, LMP-2 and CD11b labelling at the meninges.

As shown in the top photomicrographs, relative to saline treated mice enhanced immunostaining for the T-lymphocyte receptor marker $\mathrm{CD} 3$ was observed in the meninges after 2-days. Immunostaining for $\mathrm{CD} 3$ continued to dramatically increase by 7-days following LPS exposure. Paralleling CD3, saline treated animals displayed minimal LMP-2 staining at the meninges. However, a 2-day delay following LPS increased immunostaining of LMP-2 and this effect was appreciably enhanced by the 7-day interval. In contrast, the most robust $\mathrm{CD} 11 \mathrm{~b}^{+}$immunostaining observed in the meninges was apparent 2-days after LPS but still moderately elevated by 7-days, relative to saline treated controls. 
$I L-6$ and $T N F-\alpha$

These two macrophage derived cytokines varied as a function of LPS treatment, $F_{\mathrm{S}_{6,33}}=4.53$ and $9.93, p<0.001$, respectively. As shown in Figure 3.3 and confirmed by the follow-up comparisons, a significant and dramatic elevation of circulating TNF- $\alpha$ and IL-6 ( $<29$ and 36-fold respective increases) was evident in mice that had received LPS 90 min earlier, relative to the remaining groups. Interestingly, the magnitude of these cytokine elevations was greater following administration of the lower $(0.1 \mu \mathrm{g})$ of the two LPS doses. Paralleling the peripheral changes, a similar elevation of TNF- $\alpha$ and IL-6 occurred within the SNc, $F \mathrm{~s}_{6,70}=4.72,4.40$, respectively, $p<0.01$. As shown in Figure 3.3 and confirmed by the follow up comparisons, an elevation of TNF- $\alpha$ and IL- 6 within the SNc occurred 90 min following LPS infusion $(p<0.01)$.

The pattern of alterations of these cytokines within the striatum following LPS exposure was clearly distinct from that evident within the SNc, such that concentrations of TNF- $\alpha$ were not significantly affected, whereas IL- 6 variations were apparent, $F_{6,24}=$ $6.01, p<0.001$. Indeed, the follow up comparisons revealed elevated levels of IL-6 within the striatum, peaking after $90 \mathrm{~min}$ and remaining elevated 2 and 7 days following the endotoxin.

\section{$I L-2$ and $I F N-\gamma$}

The overall ANOVA indicated that plasma levels of IFN- $\gamma$ were influenced by the intra-SNc infusions, $\mathrm{F}_{6,68}=2.71, p<0.05$. Although levels of IFN- $\gamma$ did not differ from saline treated controls at the $90 \mathrm{~min}$ interval, the cytokine was significantly elevated 7 days following the higher $3.0 \mu \mathrm{g}$ dose of LPS $(p<0.05)$. As shown in Figure 3.3, a similar time dependent change was apparent for circulating IL-2, although the ANOVA was just shy of significance $\mathrm{F}_{6,56}=2.10, p=0.06$. Nonetheless, based upon previous 
evidence indicating disturbed T-lymphocytes responses in PD (Baba et al., 2005), it had been hypothesized that both of the Th-1 associated cytokines, IFN- $\gamma$ and IL-2, would be altered by the intra-SNc LPS infusion. Based on the a priori hypothesis, follow-up comparisons were conducted. These comparisons revealed that IL-2 was significantly reduced 90 min following LPS infusion, but increased with the passage of time, such that levels of the cytokine exceeded that of controls at 7-days following the high dose of the endotoxin ( $p<0.05$; see Figure 3.3). In contrast to the peripheral variations, $\mathrm{SNc}$ and striatal levels of IL-2 and IFN- $\gamma$ were not altered by intra-SNc LPS treatment.

Insert Figure 3.3 about here

Experiment 2: LPS sensitized or de-sensitized DA neurons to paraquat depending upon timing of exposure.

Experiment 1 established some of the inflammatory immune changes provoked by intra-SNc LPS that might influence neuronal vulnerability. For the present experiment, we hypothesized that the inflammatory state of the $\mathrm{SNc}$ microenvironment would determine whether DA neurons were more or less vulnerable to subsequent paraquat exposure. Thus, mice were exposed to paraquat 2- or 7-days following intra-SNc LPS; at times corresponding to robust microglial and immune cell responses.

The ANOVAs revealed that the number of $\mathrm{TH}^{+}$neurons varied as a function of the treatments, with a statistically significant LPS infusion $\times$ paraquat interaction at two different SNc levels, $F \mathrm{~s}_{1,35}=4.18 ; 6.30, p<0.05$, bregma levels -3.08 and -3.40 , respectively. As shown in Figure 3.4, chronic systemic exposure to paraquat or acute intra-SNc infusion of LPS caused a modest $(<30 \%)$, yet statistically significant, reduction 

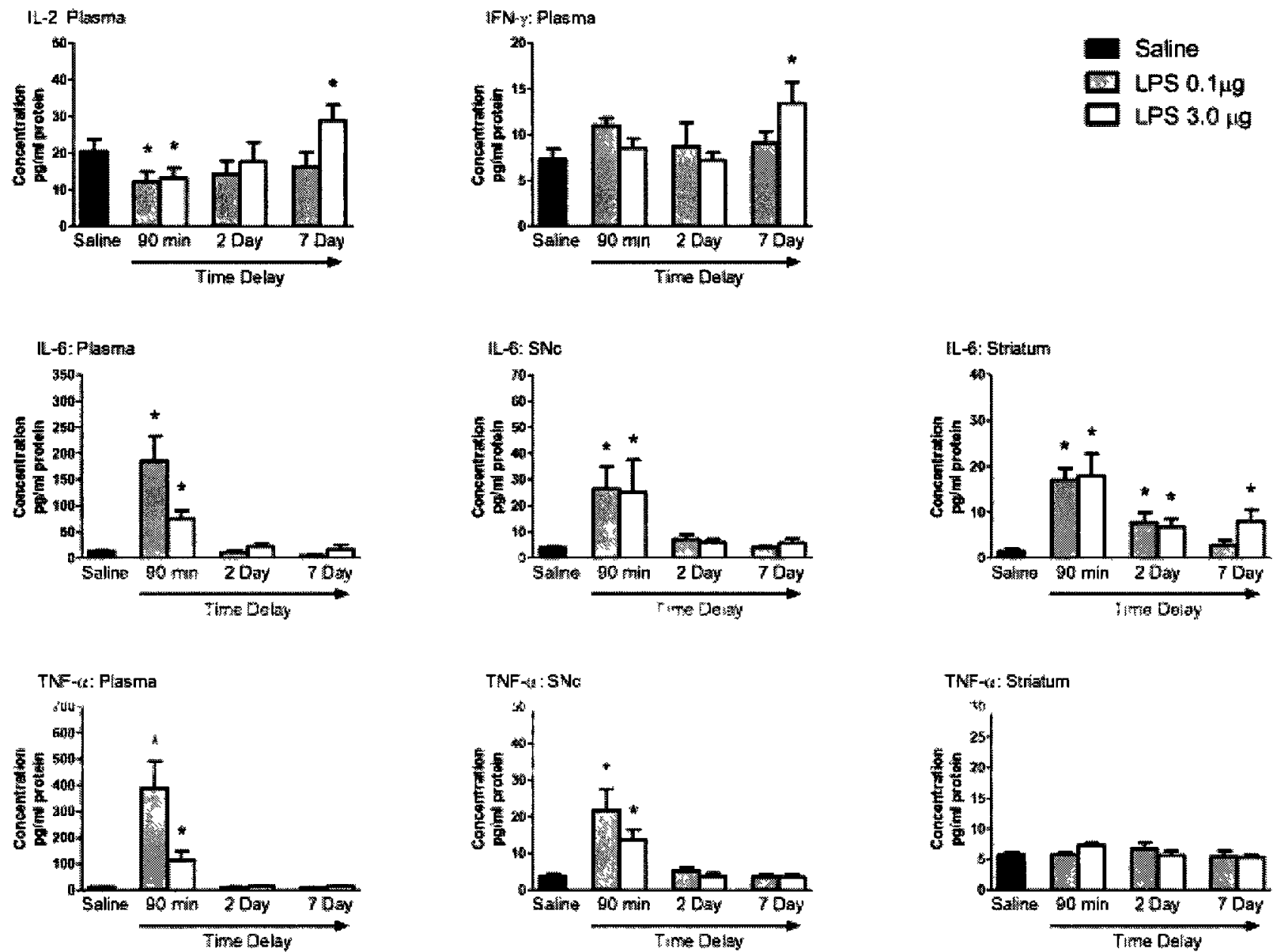

Figure 3.3 Intra-SNc LPS infusion provoked marked time-dependent elevations in several pro-inflammatory cytokines.

LPS induced profound elevations in IL-6 (peaking after $90 \mathrm{~min}$ ) relative to saline treatment within plasma, SNc and striatum. Interestingly, the rise in IL-6 persisted for the entire 7-day interval within the striatum, at least following the high dose of LPS. Paralleling the IL-6 changes, LPS provoked a dramatic elevation of plasma and SNc levels of TNF- $\alpha$ after the $90 \mathrm{~min}$ interval, with the $0.1 \mu \mathrm{g}$ dose being somewhat more effective. However, intra-SNc LPS infusion did not influence TNF- $\alpha$ level within the striatum. Interestingly, the two T/NK cell derived inflammatory cytokines, IL-2 and IFN$Y$, displayed similar patterns of elevation, wherein their levels were increased within plasma only at the 7-day interval following the 3.0 LPS injection. In contrast to this later elevation, both LPS doses reduced plasma IL-2 levels after $90 \mathrm{~min}$, relative to saline administration. The effects of LPS upon IL-2 and IFN- $\gamma$ appear to have been limited to the periphery since levels of these cytokines within the $\mathrm{SNc}$ and striatum were not affected by LPS. $p<0.05$, relative to intra-SNc saline infusion. 
in the number of $\mathrm{TH}^{+}$cells across levels of the $\mathrm{SNc}$, relative to saline treated controls $(p<$ $0.05)$.

However, animals that were pre-treated with the bacterial endotoxin 2-days prior to receiving the paraquat injections were much more vulnerable to the impact of the pesticide. Indeed, these mice displayed an additional 35-60\% reduction of $\mathrm{TH}^{+}$labelling, such that the LPS pre-treatment resulted in a significantly greater loss of neurons being induced by paraquat compared to animals that had received the pesticide in the absence of the endotoxin $(p<0.05 ;$ Figure 3.4$)$.

Corroborating the sensitized loss of $\mathrm{TH}^{+}$neurons, the mice treated with LPS and then paraquat also displayed FluoroJade B staining within the SNc (Figure 3.4). Presence of this marker for dying neurons in this group, but not in any of the other treatment conditions, suggests that an ongoing neurodegenerative process may be selectively activated in the sensitized mice. In contrast to these sensitized effects after the 2-day interval, LPS pre-treatment 7-days prior to paraquat exposure did not augment the impact of the pesticide upon SNc neurons, suggesting that the effects of the inflammatory endotoxin may have begun to dissipate after several days or that alternate factors were induced (Figure 3.4).

Insert Figure 3.4 about here

In a subset of SNc tissue samples ( $n=5$ /group), double labelling was conducted to determine whether the LPS and paraquat induced loss of $\mathrm{TH}^{+}$neurons was specific for these neurons, rather than reflecting a global neurodegenerative response. In this regard, 
Figure 3.4 LPS time-dependently affected the sensitivity of SNc dopaminergic neurons to the effects of paraquat.

Paraquat alone (i.p.; $10 \mathrm{mg} / \mathrm{kg} 3$ times a week for 3 weeks) provoked a loss of $\mathrm{TH}^{+} \mathrm{SNc}$ neurons and this effect was considerably enhanced in mice that were pre-treated with LPS $(0.01 \mu \mathrm{g}) 2$-days prior to receiving paraquat. Moreover, as observed in the above photomicrograph, the surviving neurons of mice treated with LPS + paraquat were morphologically abnormal in appearance relative to animals that received saline. Quantification of number of $\mathrm{TH}^{+}$neurons, which is presented in the bar graphs at the middle left, confirmed that LPS pre-treatment 2-days prior to paraquat resulted in a greatly augmented loss of neurons at within the SNc. The far right photomicrographs depict FluoroJade B immunohistochemistry of mice infused with LPS 2-days prior to commencing paraquat. FluoroJade B immunostaining was only present in the ipsi-lateral side of the brain, near the injection site. Finally, the bottom left confocal photomicrograph $(20 \times$ magnification) depicts double labelled SNc sections from mice pre-treated with LPS that received saline or paraquat 2-days later. The number of neurons that co-expressed the NeuN (green) and TH (red) was reduced in the LPS-paraquat treated mice (arrows give example of $\mathrm{NeuN}^{+} / \mathrm{TH}^{+}$neurons). However, there was no difference between the groups with respect to the population of $\mathrm{NeuN}^{+}$neurons that did not express $\mathrm{TH}\left(\mathrm{NeuN}^{+} / \mathrm{TH}^{-}\right.$; see arrowheads for example). The lower right bar graph shows the quantification of these neuronal populations. ${ }^{*} \mathrm{p}<0.05$, relative to salinesaline treated controls. 

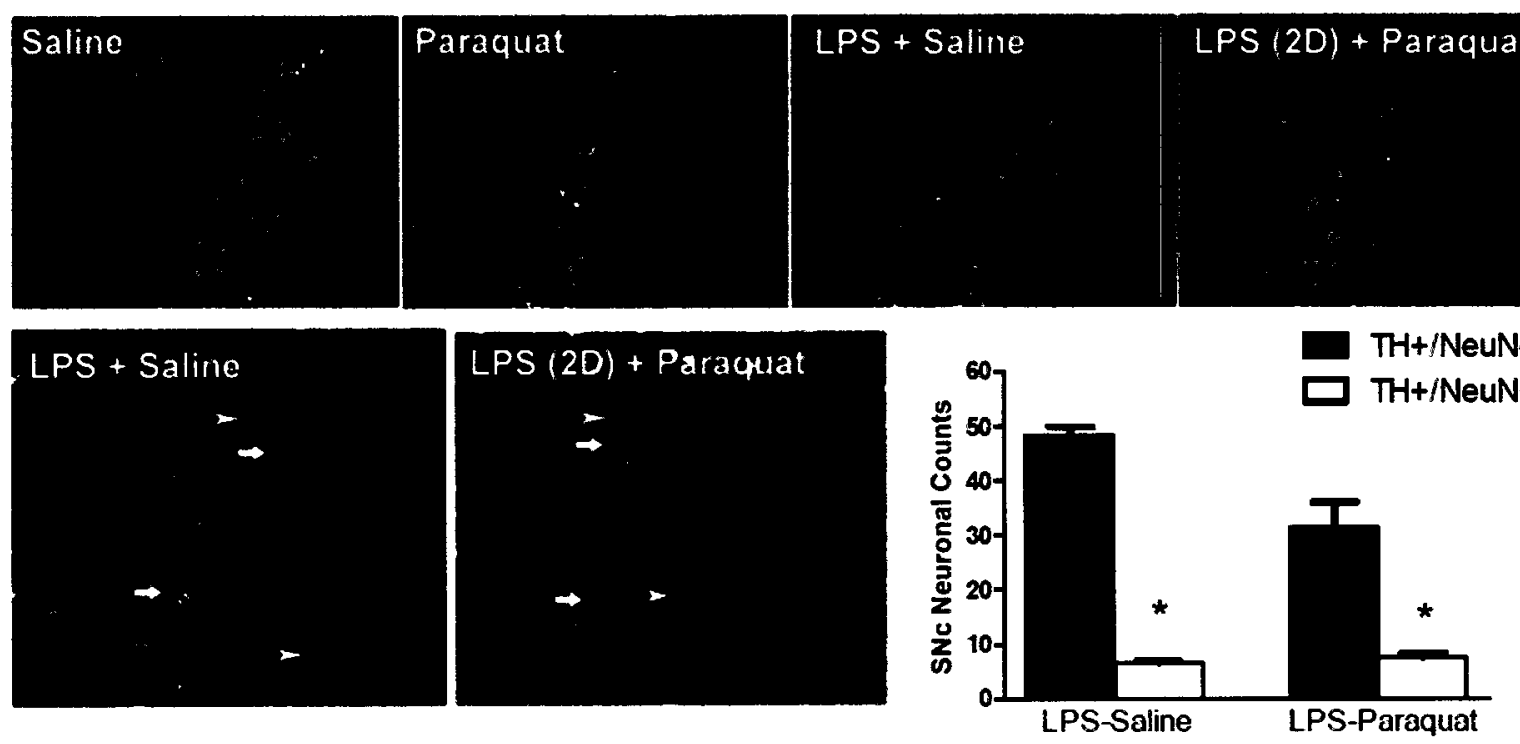

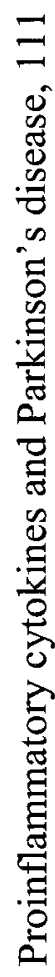

Bregma: -3.08

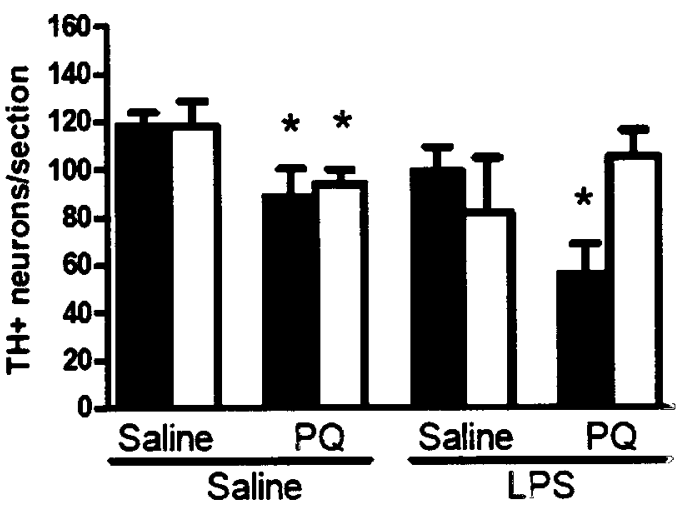

Bregma: -3.40

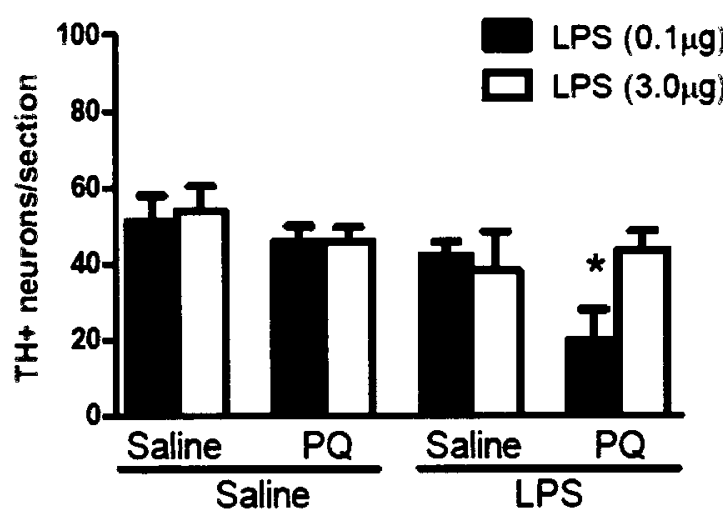

Contra-lateral

LPS (7D) + Paraquat
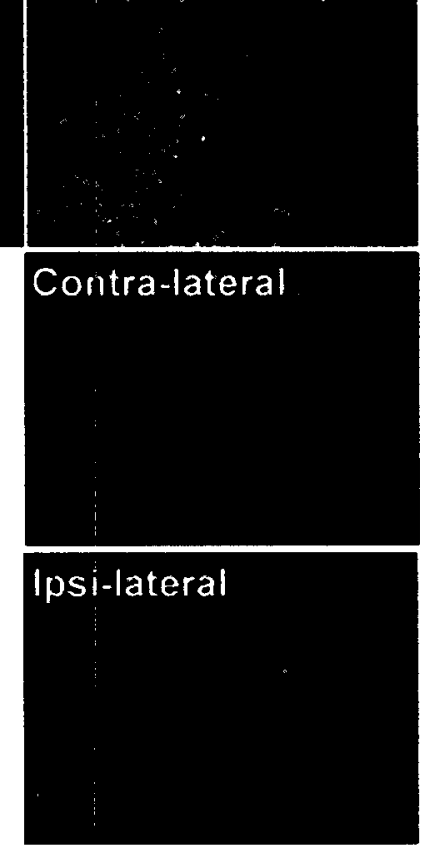
quantification of double labelled $\left(\mathrm{TH}^{+}\right.$and $\left.\mathrm{NeuN}^{+}\right)$confocal images revealed a significant reduction of $\mathrm{TH}^{+}$neurons that co-express $\mathrm{NeuN}^{+}$in LPS primed mice that received paraquat treatment 2-days later, relative to mice that had received saline following the endotoxin, $F_{1,8}=14.23, p<0.01$. However, there was no difference between these groups in the number of NeuN expressing neurons that did not express $\mathrm{TH}\left(\mathrm{NeuN}^{+} / \mathrm{TH}^{-}\right)$, suggesting that the LPS and paraquat treatments did not affect this sub-population. As well, the surviving $\mathrm{TH}^{+}$neurons of the LPS-paraquat treated mice appeared morphologically impaired, often displaying intracellular vacuoles and poor cellular definition, raising the possibility that these "sick" neurons may degenerate after more protracted delays.

In addition to the SNc variations, paraquat treatment significantly reduced striatal $\mathrm{TH}^{+}$terminal density, $F_{1,21}=17.85, p<0.05$. Although there was no significant LPS treatment main effect or interaction with the paraquat treatment, our a priori hypothesis was that LPS pre-treatment would modify the impact of paraquat. Thus, we conducted post-hoc comparisons of mice that received paraquat in the presence or absence of LPS pre-treatment. Interestingly, as shown in Figure 3.5, LPS pre-treatment actually diminished the impact of paraquat exposure 7-days later, indicating that the endotoxin had a de-sensitizing effect at this interval.

Densitometry measures confirmed that $\mathrm{TH}^{+}$terminal staining within the striatum were significantly greater in LPS pre-treated mice that received paraquat 7-days later, compared to those that received the pesticide following saline pre-treatment, $p<0.05$; $<46 \%$ elevation in LPS-paraquat 7-day animals, see Figure 3.5. In effect, the 7-day LPS 
pre-treatment interval appeared to preserve the striatal terminals, attenuating the impact of paraquat, whereas no such effect was observed at the 2-day interval.

Insert Figure 3.5 about here

Inflammatory $S N c$ glial changes that accompany the LPS-paraquat provoked neurodegeneration

Enhanced SNc microglial reactivity was provoked by the individual LPS and paraquat treatments. Specifically, among mice that received intra-SNc LPS infusions $\mathrm{CD}_{1} \mathrm{~b}^{+}$cells were more numerous than in animals that received saline infusion (Figure 3.6). It is noteworthy that these animals had received the LPS infusion more than a month earlier, indicating that a single exposure to the endotoxin provoked long-term microglial changes, albeit of a smaller magnitude than observed after shorter intervals (see Experiment 1). Similarly, a modest elevation of CD11b reactivity within the SNc was observed following the 3 weeks of paraquat injections compared to mice that only received saline (Figure 3.6). However, the most prominent changes in CD11b immunoreactivity occurred in intra-SNc LPS pre-treated mice that received paraquat 2days later. Indeed, these mice displayed microglial cells characteristic of a highly activated state with shortened thick dendritic processes and compact round amoeboid-like soma similar to that of peripheral macrophages (Figure 3.6). In contrast, $\mathrm{CD}_{11} \mathrm{~b}^{+}$ labelling was not appreciably influenced by LPS pre-treatment 7-days prior to paraquat administration, such that the microglial marker did not differ between these animals and those that received the pesticide in the absence of the endotoxin. Thus, paralleling the sensitized loss of $\mathrm{TH}^{+}$neurons, LPS appeared to provoke a time-dependent sensitization 
Figure 3.5 Intra-SNc infusion of LPS and systemic paraquat time-dependently affected striatal dopaminergic terminal.

The photomicrographs depict immunostaining of $\mathrm{TH}^{+}$terminals within the striatum in mice that received either saline or LPS $(0.01 \mu \mathrm{g})$ followed by a 2- (top panels) or 7(bottom panels) day delay later prior to commencing saline or paraquat treatments (i.p.; $10 \mathrm{mg} / \mathrm{kg}$ three times a week for 3 weeks). Mice that received paraquat displayed reduced $\mathrm{TH}^{+}$terminal staining relative to their respective controls. In contrast, LPS followed by saline 2- or 7- days later did not influence $\mathrm{TH}^{+}$labelling of striatal terminals. Mice that received paraquat 2-days following LPS pre-treatment displayed a diminution of $\mathrm{TH}^{+}$ staining comparable to that observed in animals exposed paraquat alone. However, LPS pre-treatment 7-days prior to paraquat administration prevented $\mathrm{TH}^{+}$reduction associated with paraquat, such that immunostaining was now similar to that of controls. Quantification of striatal density depicted in the bar graph at the bottom confirmed that these findings. ${ }^{*} p<0.05$. 

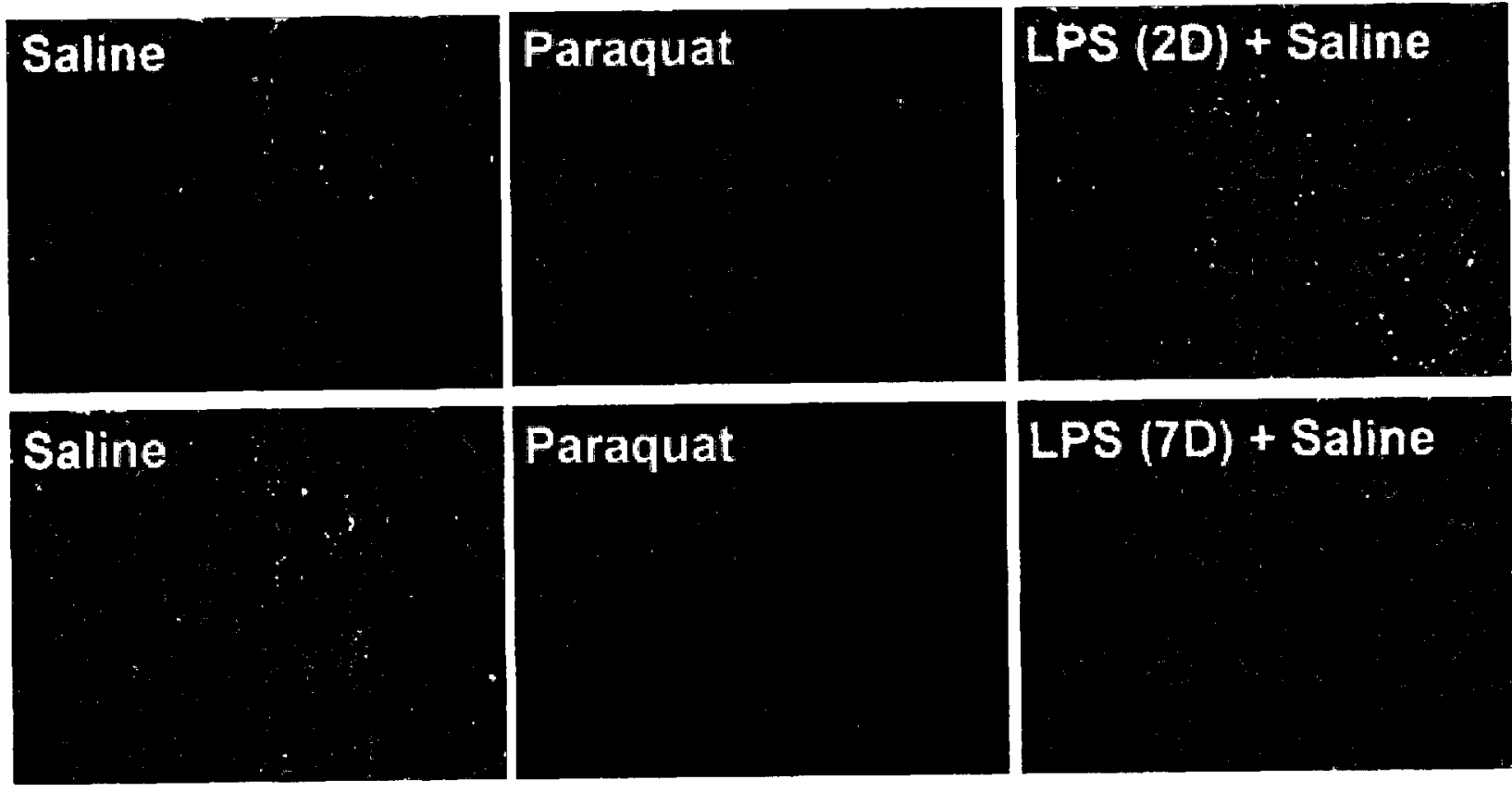

LPS (7D) + Rarauqat

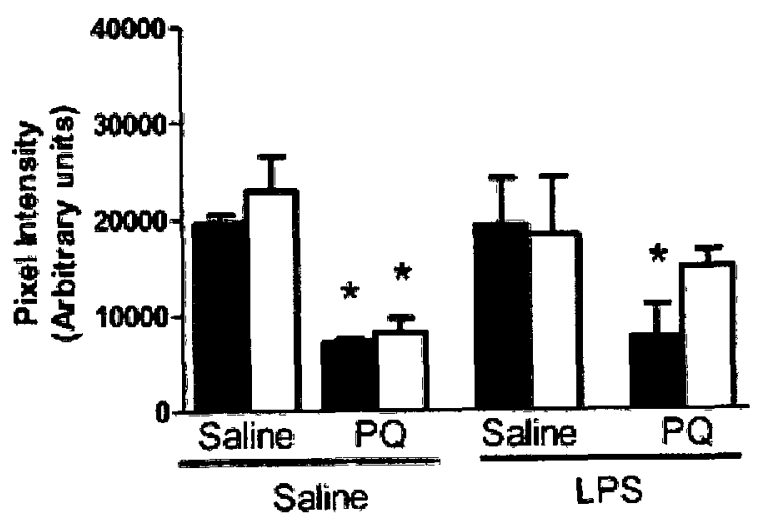

2 Day Delay

口 7 Day Delay

LPS (2D) + Paraquat

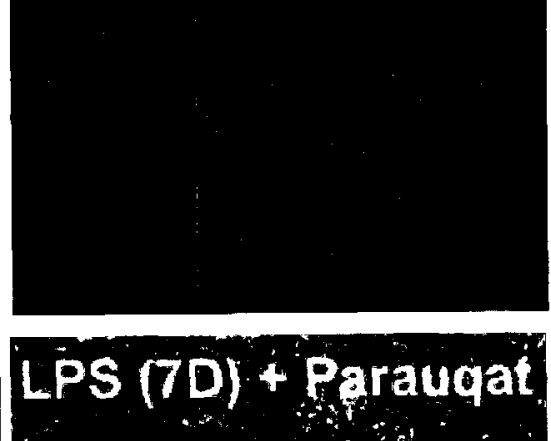

(7)

. 
of SNc microglial reactivity to later paraquat exposure that was restricted to the 2-day interval.

In contrast to the elevation of microglial staining provoked by paraquat treatment, astrocyte expression within the $\mathrm{SNc}$, as indicated by $\mathrm{GFAP}^{+}$immunoreactivity, was actually reduced in mice exposed to the pesticide (Figure 3.6). Moreover, mice that were pre-treated with LPS 2-days prior to paraquat displayed an even greater reduction of $\mathrm{GFAP}^{+}$labelling, but no such effect was apparent after the 7-day interval (Figure 3.6). Since the $\mathrm{GFAP}^{+}$reduction was quite profound and not entirely expected, this finding was further confirmed by Western blot analyses. As shown in Figure 3.6, the extent of the $\mathrm{GFAP}^{+}$reduction was so profound that the astrocyte marker was virtually undetectable in the LPS primed mice that received paraquat 2-days later. Thus, it seems that a desensitization of astrocyte expression accompanied the LPS-paraquat sensitized microglial response. However, it is underscored that reduced $\mathrm{GFAP}^{+}$expression does not necessarily imply that astrocyte functioning was impaired. Indeed, substantial heterogeneity of astrocyte responses has been reported following several types of CNS injury, such as stab-wounds, axotomy and ischemia. Moreover, besides GFAP ${ }^{+}$, assessment of other phenotypic characteristics of reactive astrocytes, including morphological aspects and expression of inflammatory factors would provide a more complete picture of the functional state of these glia (Faulkner et al., 2004; Gordon et al., 1997; Krum et al., 2002; McKinnon and Margolskee, 1996).

Insert Figure 3.6 about here 
Figure 3.6 Intra-SNc infusion of LPS and systemic paraquat time-dependently affected glial expression in the $S N C$.

The photomicrographs depict $\mathrm{CD}_{1} \mathrm{~b}^{+}$and $\mathrm{GFAP}^{+}$immunostaining within the $\mathrm{SNc}$ of mice that received either saline or LPS $(0.01 \mu \mathrm{g})$ followed by saline or paraquat treatments (i.p.; $10 \mathrm{mg} / \mathrm{kg} 3$ times a week for 3 weeks). Paraquat but not LPS treated mice displayed modestly elevated $\mathrm{CD}_{1} 1 \mathrm{~b}^{+}$immunostaining, relative animals that received only saline. However, marked $\mathrm{SNc} C D 11 b^{+}$immunostaining was evident in mice that received paraquat 2-days after LPS exposure. Yet, $\mathrm{CD} 11 \mathrm{~b}^{+}$staining resembled that of saline treated mice if LPS pre-treatment occurred 7-days prior to paraquat. In contrast $\mathrm{GFAP}^{+}$immunoreactivity within the $\mathrm{SNc}$ was diminished by paraquat exposure but was unaffected by LPS, relative saline treatment. However, the most pronounced changes occurred in mice that were primed with LPS and then exposed to paraquat 2days later, such that GFAP ${ }^{+}$staining was markedly reduced. $\mathrm{GFAP}^{+}$was still moderately reduced when paraquat exposure commenced 7-days following administration of the endotoxin. The profound $\mathrm{GFAP}^{+}$reduction observed in the LPS-paraquat (2-day) treated mice was further confirmed by Western blot analyses, such that GFAP ${ }^{+}$expression was virtually undetectable in these animals. 


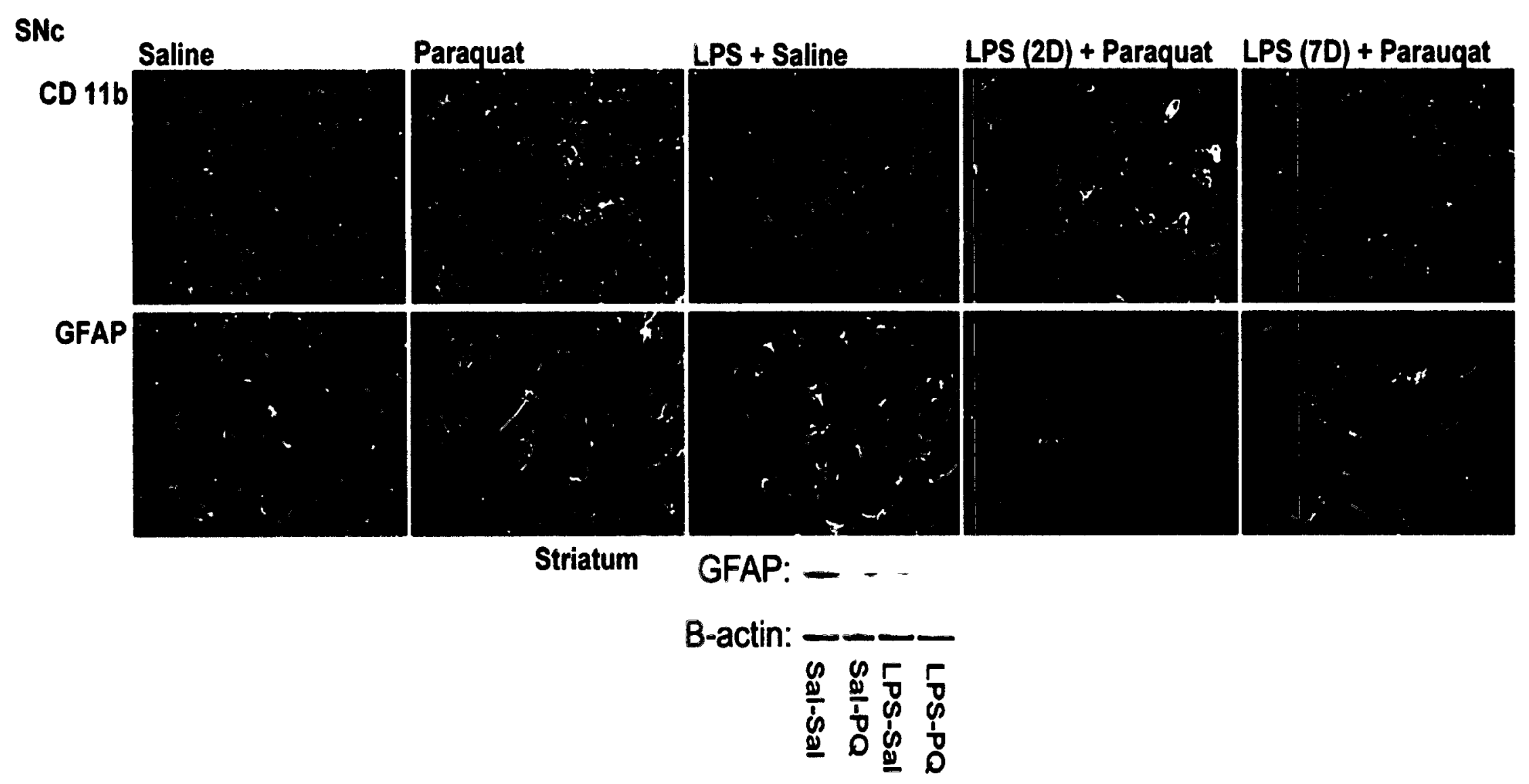


Striatal glial and FGF changes accompany the LPS-paraquat treatments

In contrast to the marked elevation of $\mathrm{CD} 11 \mathrm{~b}^{+}$staining observed within the $\mathrm{SNc}$, the LPS and paraquat treatments had little effect on this microglial marker within the striatum. However, paralleling the $\mathrm{GFAP}^{+}$reduction observed in the SNc, Western blot analyses indicated that following exposure to paraquat the expression of this astrocyte marker was likewise diminished within the striatum (Figure 3.7). Interestingly, LPS pretreatment 7-days prior to paraquat actually attenuated the impact of the pesticide, such that $\mathrm{GFAP}^{+}$expression was now comparable to that of saline treated controls. Striatal $\mathrm{FGF}^{+}$immunoreactivity was also assessed in order to determine whether this growth factor might have been associated with the preservation of $\mathrm{TH}^{+}$terminals promoted by LPS pre-treatment. Indeed, mice that received LPS pre-treatment 7-days before paraquat displayed markedly greater $\mathrm{FGF}^{+}$immunoreactivity within the ipsi-lateral striatum (Figure 7) compared to that evident in the remaining groups. The majority of $\mathrm{FGF}^{+}$cells were punctate in appearance (see inset Figure 3.7); however, cytoplasmic staining was evident in a few cells resembling neurons that were scattered sparsely throughout the striatum. Thus, it seems that endotoxin pre-treatment 7-days prior to paraquat ameliorated the $\mathrm{GFAP}^{+}$reduction associated with the pesticide and promoted enhanced striatal expression of FGF in the absence of any $\mathrm{CD} 11 \mathrm{~b}^{+}$changes.

Insert Figure 3.7 about here

\section{LPS and paraquat treatments provoked behavioural impairment}

Assessment of posture, gait and muscle tone was made by 2 independent raters, blind to the treatment conditions, $1-\mathrm{hr}$ following the final experimental injection. The 


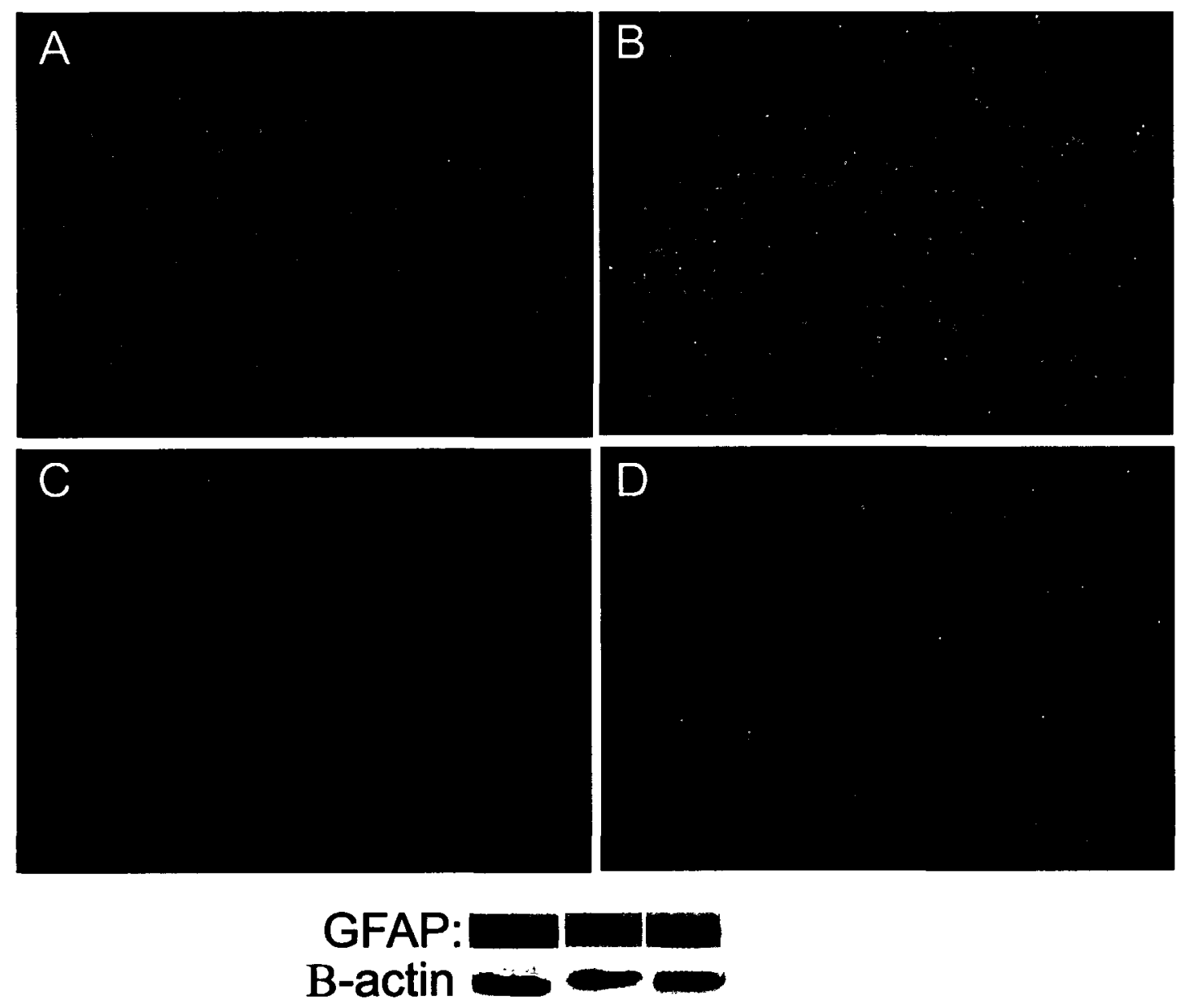

Figure 3.7 A 7-day delay between LPS and paraquat modulated glial response within the striatum to promote trophic support.

$\mathrm{CD} 11 \mathrm{~b}^{+}$immunostaining within the striatum was not appreciable influenced by the LPS or paraquat treatments. For instance, saline treated mice (A) did not differ in CD11 $\mathrm{b}^{+}$staining compared to those that received the LPS and paraquat treatments 7-days apart (B). However, mice that were administered paraquat 7-days following the endotoxin did display elevated levels of the growth factor, FGF (D), relative to their saline injected counterparts (C). Striatal GFAP expression (E) was significantly diminished following a 2-day delay between LPS and paraquat (lane 2), relative to animals that were administered saline only (lane 1). However, a 7-day delay between LPS and paraquat exposure attenuated the impact of the pesticide (lane 3), such that GFAP expression in these mice was returned to control levels. 
ANOVAs of these outcome variables revealed significant main effects for LPS infusion, paraquat injection and time between these treatments, $F s_{1,82}=17.54,26.91$ and $6.33, p<$ 0.01 , respectively. Moreover, the LPS infusion $\times$ time interaction was just shy of significance, $F_{1,82}=3.41, p=0.06$. Essentially, animals that received the paraquat or LPS infusion alone had modest signs of motor impairment, receiving intermediate, but statistically significant, ratings of disturbed gait, posture and muscle tone, relative to saline treated controls $(p<0.05$; Figure 8$)$. However, LPS pre-treated mice that were later exposed to paraquat appeared the most severely compromised group, with hunched posture, profoundly splayed hind-limbs and when placed in an open field had a clearly slow shuffling gait, characterized by a start-stop pattern of movement and dragging of hind limbs. Of these LPS pre-treated mice, those that received the paraquat regimen 2days after the endotoxin had the highest ratings of motor impairment, with ratings exceeding that of animals that received the individual LPS or paraquat treatments $(p<$ 0.05; Figure 3.8).

Insert Figure 3.8 about here 
Figure 3.8 Toxin induced behavioral changes.

Among saline pre-treated mice (left half of the graph), later paraquat (PQ) administration provoked elevated ratings of overall motor impairment. Similarly, mice that received intra-SNc LPS infusion followed by saline treatment also had ratings of motor impairment that were significantly above those of control animals (those receiving saline on both occasions). However, the most pronounced signs of motor dysfunction were evident in mice pre-treated with LPS and later exposed to paraquat (far right bars), such that these animals displayed marked postural instability, disturbances of gait (startstop shuffling pattern of movement) and abnormal muscle tone. Moreover, these motoric disturbances were most pronounced in LPS pre-treated mice that received the paraquat treatments 2 days later (black bars). ${ }^{*} p<0.05$, relative to saline-saline treated controls, $p$ $<0.05$, relative to the respective saline-paraquat treated mice. 
Proinflammatory cytokines and Parkinson's disease, 123
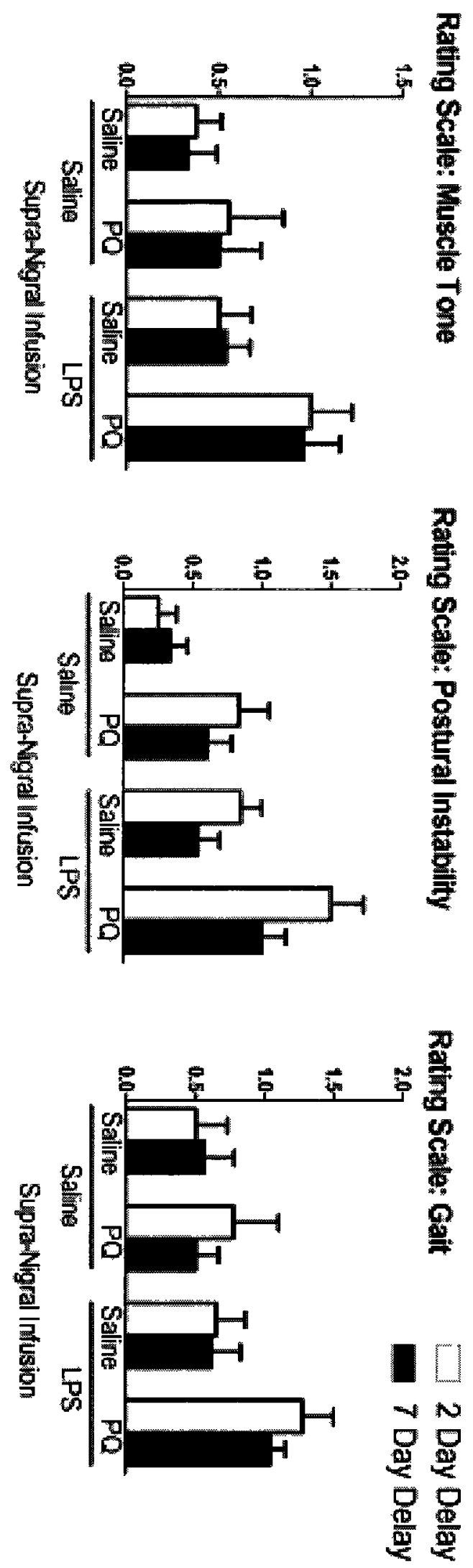


\section{Discussion}

Sensitization of neurodegenerative effects of LPS and paraquat (2 days)

Consistent with the notion those environmental toxins may contribute to PD

(Betarbet et al., 2002; Di Monte, 2003; McCormack et al., 2002), paraquat provoked a loss of SNc TH+ neurons. As previously reported (Cicchetti et al., 2005; Ossowska et al., 2005b; Peng et al., 2004; Saint-Pierre et al., 2006), the loss of $\mathrm{TH}^{+}$neurons of the SNc, although statistically significant, was relatively modest (approximately $15-30 \%$ across multiple SNc levels) compared to the marked loss of DA neurons ordinarily evident in PD patients. However, when mice were pre-treated with the inflammatory agent, LPS, 2days prior to paraquat exposure, the degree of $\mathrm{TH}^{+}$soma loss was greatly augmented (by $<40-60 \%$ ), and marked behavioural impairment was apparent. The LPS-paraquat sensitization appeared to be relatively specific for DA neurons, as these treatments did not significantly affect non- $\mathrm{TH}^{+}$mature neurons.

Moreover, the LPS-paraquat (2-day) treatment also induced FluoroJade B staining of the SNc, raising the possibility of ongoing degeneration in surviving neurons. Hence, an even greater degree of degeneration might have been evident after more protracted intervals. In effect, endotoxin priming sensitized SNc DA neurons to the impact of the later paraquat challenge. This is in keeping with the concept of a "multiple hit" hypothesis, in which exposure to an array of environmental or inflammatory insults may precipitate pro-death pathways in nigrostriatal neurons (Carvey et al., 2006; Ling et al., 2006). As reported following MPTP exposure (Eberling et al., 1997; Saporito et al., 1999;

Sriram et al., 2006), paraquat induced a loss of $\mathrm{TH}^{+}$striatal terminals that was appreciably greater than that observed for SNc cell bodies. However, unlike the 
sensitized loss of SNc soma promoted by LPS, pre-treatment with the endotoxin 2-days earlier did not further enhance the impact of paraquat on $\mathrm{TH}^{+}$striatal terminals.

It seems that although DA terminals were more sensitive to paraquat itself, the cell bodies were especially vulnerable to the pesticide only after endotoxin priming. Such differences in sensitivity may stem from the route of administration of the toxins (i.e. systemic paraquat vs. intra-SNc LPS), as well as the fact that, like MPTP, paraquat appears to affect the DA transporter located on $\mathrm{TH}^{+}$striatal terminals, whereas LPS interacts with toll like receptor 4 (TLR4) and CD14 that are selectively found on microglia (Lehnardt et al., 2003; Ossowska et al., 2005a). Given the particularly high density of microglia found at the SNc (in the direct vicinity of the LPS infusion), it might be expected that the endotoxin would have particularly marked inflammatory effects at the level of the soma.

Sensitized and de-sensitized glial responses associated with LPS and paraquat

Microglial activation accompanied the neurodegenerative effects of LPS and paraquat, as reported following exposure to MPTP (Czlonkowska et al., 2002; Teismann et al., 2003). Indeed, the LPS sensitized loss of SNc DA neurons was associated with enhanced staining of amoeboid-like microglia (indicative of a highly active state) expressing the complement 3 receptor component (CD11b) and the immunoproteasome subunits (LMP-2 and -7). Since these LMP subunits ordinarily organize the proteasome for processing of antigens following viral or endotoxin exposure (Fehling et al., 1994; Marques et al., 2004; Van Kaer et al., 1994), it is possible that the LPS pre-treatment may have augmented microglial responses to or processing of paraquat (as an antigen). In fact, microglia that co-express CD11b and LMPs may be in a highly activated state primed to release a host of inflammatory and oxidative (e.g. superoxide) factors that may 
be damaging to local DA neurons (Hanisch, 2002; Herrera et al., 2005; Montine et al., 2002; Shie, 2005). Indeed, aberrant LMP-2 and LMP-7 expression was reported in Huntington's and Alzheimer's disease brains (Diaz-Hernadez et al., 2003; Mishto et al., 2006) . However, this is the first report showing their induction within the SNc, and specifically within microglia.

In contrast to the robust microglia response evident following LPS-paraquat treatment, astrocyte reactivity (GFAP) was reduced within the SNc of these mice, raising the possibility that these different glial populations might have opposing effects upon DA neuronal vulnerability. Indeed, unlike microglia, astrocyte derived growth factors were reported to protect midbrain DA neurons from LPS and the MPTP metabolite, MPP ${ }^{+}$ (Chen et al., 2006), and an anti-inflammatory drug protected DA neurons from LPS by stimulating astrocytes while concomitantly having inhibitory actions upon microglia (Zhang, 2005). However, reactive astrocytes have also been associated with negative consequences, including impairment of re-myelination, propagation of seizures and in certain instances; treatments aligned with a down-regulation of the astrocyte response may have neuroprotective consequences (Fawcett and Asher, 1999; Kawahara et al., 2002). Nevertheless, we speculate that the sensitized microglial response, coupled with a down-regulation of astrocytes (GFAP expression) could potentially enhance DA neuronal vulnerability.

\section{Cytokine variations provoked by LPS pre-treatment}

Consistent with divergent cytokine and microglial responses previously reported (Ling et al., 2006), intra-SNc LPS infusion induced peak IL-6 and TNF- $\alpha$ responses much earlier than the glial variations, suggesting that the cytokines may have been acting early in the inflammatory response, however, IL-6 was still modestly (but significantly) 
elevated within the striatum at 2-and 7-days following LPS. Genetic deletion of this cytokine was previously shown to enhance vulnerability to the neurodegenerative, as well as microglial and iNOS responses, provoked by MPTP (Bolin et al., 2002; Cardenas and Bolin, 2003), suggesting a protective role for IL-6. In contrast, TNF- $\alpha$ contributed to nigrostriatal neurodegeneration provoked by several DA insults (Ferger et al., 2004; Lee da et al., 2005; Sriram et al., 2002; Sriram et al., 2006; Takeuchi et al., 2006; Wu et al., 2002). Given that LPS can disrupt BBB permeability and recruit circulating monocytes, some brain cytokine changes might stem from infiltrating peripheral immune cells (Hartz et al., 2006; Montero-Menei et al., 1996; Xiao et al., 2001). In this regard, we found that LPS enhanced staining of CD3, LMP and CD1 lb positive cells around several ventricular and meningeal areas, presumably reflecting enhanced mobilization of immune cells. Once present within the brain, these immune cells can influence the inflammatory microenvironment by releasing cytokines or directly interacting with microglia.

\section{LPS pre-treatment diminishes the impact of paraquat 7 days later}

In contrast to the effects of LPS pre-treatment 2-days prior to paraquat, infusion of the endotoxin 7-days prior to paraquat did not influence the loss of SNc neurons associated with the pesticide, suggesting that the sensitizing effects of LPS were transient or that compensatory anti-inflammatory, metabolic or other protective processes may have been up-regulated over time. Interestingly, within the striatum, LPS pre-treatment actually attenuated the impact of paraquat administered 7-days later, suggesting that endotoxin priming at this interval may have had a neuroprotective effect upon DA terminals. The LPS pre-treatment may have acted to either prevent the paraquat-induced loss of $\mathrm{TH}^{+}$terminals, or alternatively, promoted some compensatory sprouting from 
non-degenerating terminals, as reported in MPTP-treated mice that received IL-1 $\beta$ (Ho and Blum, 1998).

Surprisingly, the enhanced striatal TH staining was not associated with any behavioural improvement, raising the possibility that the behavioural analyses reflected processes independent of DA functioning or that the degree of neuronal loss was insufficient to provoke motor impairment. Notably, however, many of the surviving $\mathrm{TH}^{+}$ SNc neurons of paraquat treated mice appeared morphologically impaired, often containing numerous intracellular vacuoles, characteristic of "sick" neurons, as reported in monkeys exposed to MPTP (Forno et al., 1994). Thus, it is possible that the $\mathrm{TH}^{+}$ terminals may have been preserved, but with a diminished functionality. Indeed, 15 days after MPTP treatment, (Bezard et al., 2000) reported that DA levels were reduced despite the fact that striatal $\mathrm{TH}^{+}$terminals remained unchanged. Others have also reported a "mismatch" between behavioural functioning and loss of $\mathrm{TH}^{+}$terminals, which may stem from compensatory time-dependent DA changes (Bezard et al., 2000; Collier et al., 2007; Robertson et al., 2004) or other neuroplastic processes following inflammatory or toxin induced injury.

As in the case of ischemic pre-conditioning, where in inflammatory insults (e.g. LPS, TNF- $\alpha$ ) elevate anti-inflammatory and growth factors, as well as protective heatshock proteins over time (Barone, 2005; Rosenzweig et al., 2004; Wang et al., 2004), similar processes might impart protective actions against paraquat exposure. Indeed, besides the enhanced striatal $\mathrm{TH}^{+}$staining, the 7-day LPS pre-treatment prevented the reduction of striatal GFAP engendered by paraquat and at the same time, enhanced expression of the growth factor, FGF, within the striatum. These data are consistent with 
the well-known positive actions astrocyte derived growth factors may have on neurons (Gomez-Pinilla et al., 1992; Gottlieb and Matute, 1999). In fact, FGF had neuroprotective consequences in DA neurons exposed to 6-OHDA and MPTP (McLay et al., 2001; Timmer et al., 2007). Besides astrocytes, immune cells, including lymphocytes and macrophages, also produce FGF (Rossini et al., 2005; Tanaka et al., 2004), raising the possibility that any infiltrating immune cells, as well as the elevated levels of the $\mathrm{T}$ cell elevations and have positive effects on DA neurons.

\section{Conclusions}

A role for inflammatory immune factors in the degeneration of DA neurons of the nigrostriatal system is supported by studies showing that anti-inflammatory compounds were neuroprotective against exposure to MPTP or pesticides (Cassarejos et al., 2006; Sriram et al., 2006), as well as intra-SNc infusion of LPS (Peng et al., 2005a; Peng et al., 2005b; Wang et al., 2004; Zhou et al., 2005). The present study suggests that modulating the inflammatory response by central immune priming may either enhance or protect against future damage associated with known DA toxins. In this regard, the timing of exposure to paraquat following LPS priming was critical in determining whether a sensitization or de-sensitization of inflammatory processes occurred. In effect, these data suggest a novel means of influencing DA neuron survival that involves modulation of glial activation, immune cell infiltration, cytokine alterations and changes in expression of LMPs and FGF. 


\section{Chapter 4}

Granulocyte macrophage-colony stimulating factor protects against substantia nigra dopaminergic cell loss in an environmental toxin model of Parkinson's disease 
Abstract

Parkinson's disease (PD) has been linked to exposure to a variety of chemical (e.g., pesticides) and inflammatory agents, which may act cumulatively over time. Finding novel means of limiting pathology associated with toxin exposure would have tremendous clinical importance. To this end, we assessed whether the hematopoietic trophic cytokine, granulocyte macrophage colony stimulating factor (GM-CSF), would inhibit the neurodegenerative effects of the pesticide, paraquat, administered either alone or following priming with the bacterial endotoxin, lipopolysaccharide (LPS). As previously observed, paraquat provoked a modest but significant neurodegenerative effect that was markedly augmented with LPS priming. Central infusion of GM-CSF into the substantia nigra pars compacta $(\mathrm{SNc})$ prevented the loss of SNc dopamine neurons to a degree comparable to that of glial derived neurotrophic factor. Importantly, systemic administration of GM-CSF also had neuroprotective consequences, suggesting that the trophic cytokine can cross the blood brain barrier to promote neuronal survival. Indeed, GM-CSF acted to inhibit the LPS and paraquat induced microglial response, while augmenting astrocyte immunoreactivity within the SNc. Moreover, GM-CSF blunted the paraquat induced reduction of brain derived neurotrophic factor within both the midbrain and hippocampus. Although paraquat reduced midbrain levels of the anti-apoptotic protein, Bcl-2, GM-CSF had no effect in this regard. Hence, GM-CSF appears to affect inflammatory and/or neuroplastic factors within the SNc that may be linked to neurodegeneration, as well as in other brain regions (hippocampus), which could be important for co-morbid non-motor symptoms in PD. These data suggest that peripheral GM-CSF administration might hold promise as a treatment of PD. 


\section{Introduction}

Epidemiological studies have revealed that chronic exposure to pesticides and other environmental toxins (e.g., heavy metals and even immune infections) are involved in the progression of Parkinson's disease (PD) (Carvey et al., 2006; Cicchetti et al., 2009; Liu et al., 2003). Parallel work in rodents has demonstrated that administration of the pesticides, paraquat and rotenone, provoked a loss of substantia nigra pars compacta (SNc) dopamine (DA) neurons, coupled with histological and behavioral symptoms reminiscent of PD (Cannon et al., 2009; Di Monte, 2003; Litteljohn et al., 2009; Sherer et al., 2007; Somayajulu-Nitu et al., 2009). The neurodegenerative process induced by paraquat, as well as other well-established DA toxins, such as MPTP, involves the activation of microglial cells and liberation of oxidative radicals, as well as proinflammatory cytokines, such as interferon- $\gamma$ (IFN- $\gamma$ ) and tumor necrosis factor- $\alpha$ (TNF$\alpha$ ) (McCoy et al., 2008; Mount et al., 2007). Indeed, the deleterious impact of paraquat was augmented in rodents pre-treated with the bacterial endotoxin, lipopolysaccharide (LPS), and this effect was associated with a heightened microglial-dependent neuroinflammatory response (Mangano and Hayley, 2009; Purisai et al., 2007). These studies are in line with post-mortem analyses of PD brain, which revealed signs of neuroinflammation and oxidative damage, coupled with mitochondrial dysfunction, and alterations of apoptotic regulators within the SNc (Fitzmaurice et al., 2003; Hartmann et al., 2002; Jenner, 1998; Knott et al., 2000; Nagatsu and Sawada, 2007; Tatton et al., 2003).

Given that the neurodegenerative process in PD occurs over a prolonged period of time, there may be a critical window wherein therapeutic intervention may halt or even reverse disease progression. In particular, the trophic cytokines, glial derived 
neurotrophic factor (GDNF) and brain derived neurotrophic factor (BDNF), have been considered possible therapeutic agents for PD (Howells et al., 2000; Porritt et al., 2005; Yasuhara et al., 2007). In fact, both BDNF and GDNF have been shown to be neuroprotective in virtually all animal models of PD (Eslamboli, 2005; Huang et al.; Hung and Lee, 1996; Levivier et al., 1995; Shults et al., 1995; Zhang et al., 2007). Unfortunately, recent clinical trials using GDNF have failed to consistently yield beneficial clinical outcomes, stemming perhaps from surgical complications and sideeffects [GDNF is unable to cross the blood brain barrier (BBB)] (Lang et al., 2006), and the limited diffusion of GDNF in human brain (i.e., leading to reduced bioavailability in nigrostriatal brain regions) (Nutt et al., 2003; Salvatore et al., 2006).

Granulocyte-macrophage colony stimulating factor (GM-CSF) is a hematopoietic cytokine produced mainly by peripheral immune cells (T-lymphocytes, macrophages, and NK cells) and also by central astrocytes (Davignon et al., 1988; Guillemin et al., 1996; Hercus et al., 2009). Its basic function is to promote the differentiation and maturation of peripheral innate immune cells, including neutrophils and dendritic cells. The role of GM-CSF in the brain is not fully appreciated; however, unlike GDNF, GM-CSF is able to cross the $\mathrm{BBB}$ and accumulate at physiologically relevant levels within the brain parenchyma (Franzen et al., 2004; McLay et al., 1997; Thomson and Lotze, 2003). Additionally, systemic exposure to GM-CSF can promote functional recovery following traumatic spinal cord injury (Bouhy et al., 2006; Huang et al., 2009) and ischemia (Nakagawa et al., 2006; Schabitz et al., 2008).

It has been suggested that GM-CSF may be neuroprotective by promoting microglia to adopt a dendritic cell-like state (Fischer and Reichmann, 2001; 
Santambrogio et al., 2001; Schermer and Humpel, 2002), facilitating the release of BDNF (Hayashi et al., 2009) and increasing the expression of anti-apoptotic proteins such as Bcl-2 (Huang et al., 2007; Kim et al., 2009a). To this end, elucidating the pro-survival mechanisms of GM-CSF against DA-targeting toxins may offer new therapeutic targets for PD. Thus, we sought to assess whether central or peripheral administration of GMCSF would prevent the neurodegenerative consequences induced by exposure to LPS followed by paraquat and whether GM-CSF's pro-survival effects are linked to changes in the glial microenvironment.

\section{Materials and methods}

C57BL/6 male mice were purchased from Charles River Laboratories (LaPrairie, Quebec, Canada) at 10-12 weeks of age and subsequently single-housed in standard polypropylene cages $(27 \times 21 \times 14 \mathrm{~cm})$, and maintained on a 12 -hr light/dark cycle. Mice were housed in a temperature $\left(21^{\circ} \mathrm{C}\right)$ and humidity controlled room, and provided with an ad libitum diet of Ralston Purina mouse chow and water. Animals were acclimatized for a period of 1 week prior to the commencement of experimental procedures. All experimental test paradigms were approved by the Carleton University Committee for Animal Care and adhered to the guidelines outlined by the Canadian Council for the Use and Care of Animals in Research.

Experiment 1: Assessment of the beneficial effects of supra-nigral administration of GDNF and GM-CSF following LPS-paraquat induced pathology.

Given that GM-CSF can diminish apoptotic cell death and stimulate the release of

BDNF, we sought to determine if a direct infusion of GM-CSF into the SNc would protect DA neurons from the neurodegenerative effects of LPS and paraquat to a degree comparable to GDNF. To this end, mice underwent stereotaxic surgery (described in an 
ensuing section) and cannulae were implanted just above the SNc. Following 1 week of convalescence, animals were infused with a single dose of either saline or LPS $(0.1 \mu \mathrm{g} / 2.0 \mu \mathrm{l})$ and given a 2 day rest period. Thereafter, mice received intraperitoneal (i.p.) injections of saline or paraquat (Sigma Aldrich; 10mg/kg), 3 times a week for 3 consecutive weeks (a paradigm previously found to reliably impact the survival of SNc DA neurons; Mangano and Hayley, 2009). At the beginning of each week (i.e., immediately prior to the $1^{\text {st }}, 4^{\text {th }}$ and $7^{\text {th }}$ paraquat or saline injection), mice $(n=16$ 20/group) received supra-SNc infusion (using the same cannulae that previously delivered LPS or saline) of saline, GDNF (R\&D Systems; $1 \mu \mathrm{g} / 2 \mu \mathrm{l}$ ) or GM-CSF (R\&D Systems; 10ng/2 $\mu \mathrm{l}$ ) into the SNc. Finally, in order to assess SNc DA neuronal survival, 5 days following the final paraquat or saline injection, a subset of mice $(n=8-10 /$ group) were perfused with $4 \%$ paraformaldehyde (PFA), brains post-fixed for 24-hrs and then cryoprotected for 3 days in a solution comprising 20\% sucrose, $0.1 \mathrm{M}$ PBS and $0.02 \%$ sodium azide ( $\mathrm{pH} 7.4)$.

\section{Immunohistochemical procedures: SNc DA neuronal survival and microglial reactivity}

Frozen brains were cryostat-sectioned and free-floating coronal sections $(20 \mu \mathrm{m})$

of the SNc were mounted onto gelatin-coated slides. Anti-tyrosine hydroxylase (TH) antibody along with $1 \%$ cresyl violet (Sigma-Aldrich) as a counter-stain was used to detect SNc DA neurons. The primary antibody (TH) was diluted in a solution containing $1 \%$ BSA, $0.5 \%$ Triton $\mathrm{X}-100,0.05 \%$ Tween 20 , and $0.05 \%$ sodium azide in $0.01 \mathrm{M}$ PBS, pH 7.3; and sections were incubated overnight at room temperature $(1: 3000$, ImmunoStar, Hudson, WI, USA). The primary antibodies were visualized using a biotin rabbit anti-mouse (1:500, Cedarlane) secondary and horseradish peroxidase-conjugated 
streptavidin tertiary (1:500, Cedarlane). Sections were then further incubated with diaminobenzidine (DAB; Sigma-Aldrich) for 10 minutes, counterstained with cresyl violet (Sigma-Aldrich) and dehydrated with serial alcohol washes before cover-slipping with clearene (Surgipath).

Survival of SNc DA-producing cells was determined by way of serial section analysis of the number of $\mathrm{TH}^{+}$cells within the $\mathrm{SNc}$, at bregma levels ranging from -2.70 to -3.88 . Using a double blind procedure, 8-10 animals were analyzed per group and the number of $\mathrm{TH}^{+}$cells was counted across multiple bregma levels. Midbrain sections from each bregma level were compared across treatment groups to assess the extent of DA neuronal loss induced by paraquat alone or primed with LPS, and to determine if GMCSF or GDNF prevented such effects. In order to ensure that a genuine loss of DA neurons occurred rather than simply a phenotypic suppression, the total number of surviving $\mathrm{SNc}$ neurons $\left(\mathrm{TH}^{+}\right.$and $\left.\mathrm{TH}^{-}\right)$was counted using cresyl violet staining. Striatal sections were stained using $\mathrm{TH}^{+}$antibody $(1: 1000)$ and photomicrographs were obtained for each animal using the same exposure time. Image $J$ software was used to determine the background threshold for each striatal section and the total number of white (background) and black $\left(\mathrm{TH}^{+}\right)$pixels.

Morphological changes of microglia are generally taken to indicate some degree of change in their activation states (Mangano and Hayley, 2009). Indeed, differing degrees of compacted soma, thickening of processes, and enhanced proliferation are normally taken to reflect a state of activation, wherein microglia are capable of substantial release of inflammatory and oxidative factors (Nimmerjahn et al., 2005). Hence, the morphological state of microglia was assessed histologically using CD11b 
(complement receptor marker present on microglia) antiserum (1:1000; AbD Serotec, Raleigh, NC, USA; overnight incubation at $4^{\circ} \mathrm{C}$ ). The primary antibody was diluted in $1 \%$ BSA, $0.03 \%$ Triton $\mathrm{X}-100,0.05 \%$ Tween 20 and $0.05 \%$ sodium azide in $0.01 \mathrm{M}$ PBS (pH 7.3). Thereafter, sections were washed with $0.01 \mathrm{M}$ PBS and incubated with biotin rabbit anti-rat secondary (1:500, Cedarlane) and horseradish peroxidase-conjugated streptavidin tertiary (1:500, Cedarlane) antibodies. Sections were once again visualized by incubation with DAB (Sigma-Aldrich) for 10 minutes and dehydrated with serial alcohol washes before cover-slipping with clearene (Surgipath).

Western Blot analyses of hippocampal BDNF levels

It has become clear that, besides the frank nigrostriatal neuronal damage, PD is associated with pathology involving brain regions important for neuroplasticity (Bruck et al., 2004; Jokinen et al., 2009). Hence, it was of interest to assess whether paraquat would affect hippocampal BDNF levels. Moreover, it is of clinical importance to evaluate whether GM-CSF administration can reverse such effects. To this end, a subset of the mice from Exp. 1 ( $n=8$-10/group) were euthanized by rapid decapitation (again 5 days after the final injection) followed by micropunch dissection of the hippocampus. Tissue samples were stored at $-80^{\circ} \mathrm{C}$ until Western blot analyses (Hayley et al., 1999; Hayley et al., 2004b).

All chemicals were obtained from Sigma-Aldrich (St. Louis, MO) and all antibodies were obtained from Santa Cruz Biotechnology (Santa Cruz, CA) unless otherwise indicated. Samples were diluted with lysis and protease inhibitor buffer up to the desired protein concentration, yielding whole cell lysate $(50 \mu \mathrm{g})$ in $20 \mu \mathrm{L}$ and $20 \mu \mathrm{L}$ loading buffer (5\% glycerol, $5 \% \beta$-mercaptoethanol, $3 \%$ SDS and $0.05 \%$ bromophenol 
blue). To denature the proteins, the $40 \mu \mathrm{L}$ sample was heated in boiling water for 5 minutes. Sodium dodecyl sulphate-polyacrylamide gel electrophoresis (SDS-PAGE), the separating buffer [370 mM Tris-base (pH 8.8), 3.5 mM SDS], and the stacking buffer [124 mM Tris-base (pH 6.8), $3.5 \mathrm{mM} \mathrm{SDS}$ ], were placed in running buffer (25 mM Trisbase, $190 \mathrm{mM}$ glycine, $3.5 \mathrm{mM}$ SDS); and samples, along with the Precision Plus Protein $^{\text {TM }}$ Standards Dual Color (Bio-Rad, Hercules, CA, Cat\#161-0374), were loaded into the Acrylamide gel (12.5\%) for molecular weight determination at $120 \mathrm{~V}$.

After electrophoresis, proteins were transferred overnight, at $4^{\circ} \mathrm{C}$ and $180 \mathrm{~mA}$, in transfer buffer (25 mM Tris-base, $192 \mathrm{mM}$ Glycine, 20\% methanol) onto PVDF (BioRad, Cat\#162-177). Thereafter, membranes were blocked for 1-hr with gentle shaking in a solution of non-fat dry milk (5\% w/v) dissolved in TBS-T buffer $(10 \mathrm{mM}$ Tris-base (pH 8.0), $150 \mathrm{mM}$ sodium chloride, $0.5 \%$ Tween-20). The membranes were then incubated with a rabbit anti-BDNF primary antibody (1:500) diluted in blocking solution at room temperature for 1-hr. Any unbound antibody was removed using three washes of $15 \mathrm{~mL}$ TBS-T at room temperature. Membranes were incubated on a shaker for $1-\mathrm{hr}$ at room temperature with HRP (horseradish peroxidase) conjugated anti-rabbit (1:2000) secondary antibody and washed again with TBS-T. Finally, BDNF was visualized with a chemiluminescent substrate (Perkin Elmer, Waltham, MA, cat\# NEL102001EA; for 1 minute) and briefly exposed on a Kodak film.

Experiment 2: Assessment of the beneficial effects of systemic administration of GM-CSF following LPS-paraquat induced pathology.

Mice underwent stereotaxic surgery identical to Experiment 1 and received a single supra-nigral infusion of either saline or LPS (same dose used in Experiment 1), followed 2 days later with i.p. saline or paraquat treatment $(10 \mathrm{mg} / \mathrm{kg} ; 3$ times per week 
for 3 weeks). Paralleling the intra-SNc infusions delivered in the previous experiment, mice received i.p. injections of GM-CSF (R\&D Systems; $2 \mu \mathrm{g}$ ) once per week for the duration of the 3 week paraquat regimen. The injection procedures are visually presented below. All mice were sacrificed 5 days after the final paraquat or saline injection and brains were perfused for immunohistochemical analyses.

\begin{tabular}{|c|c|c|c|c|c|c|c|c|}
\hline I & 6-days Rest & I & 2-days Rest & 1-day Rest & I & 1-day & 5-days & I \\
\hline $\begin{array}{l}\text { Cannulae: } \\
\text { Supra-SNc }\end{array}$ & $\begin{array}{l}\text { In } \\
\text { LI }\end{array}$ & $\begin{array}{l}\text { sion: } \\
\text { / Saline }\end{array}$ & $\left\{\begin{array}{l}\text { GM-CSF/ } \\
\text { Saline (i.p.) }\end{array}+\right.$ & $\left.\begin{array}{c}\mathrm{PQ} / \text { saline } \\
\text { (i.p.) }\end{array}\right\}$ & $\begin{array}{c}\mathrm{PQ} / \text { Saline } \\
\text { (i.p.) }\end{array}$ & $\begin{array}{c}\mathrm{PQ} / \text { Saline } \\
\text { (i.p.) }\end{array}$ & & Euthanize \\
\hline
\end{tabular}

Immunohistochemical analyses of SNc neuronal loss

Stereological analysis of SNc sections $(60 \mu \mathrm{m})$ was used to determine DA degeneration for Experiment 2. Briefly, the SNc was outlined under 2.5X magnification and $\mathrm{TH}^{+}$neurons counted using a $60 \mathrm{X}$ oil immersion objective. The SNc was sampled in a systematic random fashion according to the optical fractionator method outlined by MicroBrightField Inc. Cells were quantified in 3-dimensional counting frames using a counting grid size of $90 \times 90 \mu \mathrm{m}$ and a counting frame size of $60 \times 60 \mu \mathrm{m}$ with a $15 \mu \mathrm{m}$ dissector height and $3 \mu \mathrm{m}$ upper and lower guard zones. Only the portion of the SNc ipsilateral to the infusion site was quantified. All analyses were conducted with the counter blind to the treatment conditions.

DA neurons were sampled throughout the rostral-caudal axis of the SNc in every $2^{\text {nd }}$ section, labelled with $\mathrm{TH}$ and counterstained with $1 \%$ cresyl violet. Free floating sections were blocked in $0.3 \%$ hydrogen peroxide solution for 30 minutes, washed in 
$10 \mathrm{mM}$ PBS and then incubated overnight at $4^{\circ} \mathrm{C}$ in mouse anti-TH primary antibody (1:500 in $10 \mathrm{mM}$ PBS with $2 \% \mathrm{BSA}, 1 \%$ sodium azide and $0.5 \%$ Triton-X, Immunostar Inc.). This was followed by incubation in secondary biotin SP-conjugated Affinipure goat anti-mouse IgG (1:200 in 10mM PBS with $2 \%$ BSA, $1 \%$ sodium azide and $0.5 \%$ TritonX, Jackson Immunoresearch Laboratories) for 2-hrs at room temperature and, lastly, incubation in tertiary peroxidase-conjugated streptavidin (1:200 in $10 \mathrm{mM}$ PBS with $2 \%$ BSA and 0.3\% Triton-X, Jackson Immunoresearch Laboratories).

Following antibody incubations, sections were rinsed 3 times for 5 minutes in $10 \mathrm{mM}$ PBS. To visualize the antibody complex, sections were incubated for 5 minutes in $1 \mathrm{ml}$ (per well) of DAB $(0.2 \mathrm{mg} / \mathrm{ml})$ in $50 \mathrm{mM}$ Tris $\mathrm{HCl}$ solution. Sections were incubated for an additional 15 minutes following addition of $50 \mu \mathrm{l}$ of $6 \%$ hydrogen peroxide (in distilled water) per well. Sections were mounted on glass slides, air-dried overnight, counterstained with $1 \%$ cresyl violet, and dehydrated with serial alcohol washes. Slides were then cover-slipped and stored at room temperature. $\mathrm{TH}^{+}$cells were quantified as outlined above and representative photomicrographs depicting $\mathrm{TH}^{+}$neurons in the $\mathrm{SNc}$ were taken using an Olympus BX52 microscope under magnification of the 10X objective for qualitative analysis. The same free-floating sections used to determine $\mathrm{TH}$ loss were also used to confirm that neurons were actually lost and $\mathrm{TH}$ expression not simply reduced. Hence, the total number of SNc neurons was determined using the cresyl violet counterstain.

\section{Assessment of glial changes}

Free-floating sections were incubated overnight at $4^{\circ} \mathrm{C}$ with either mouse antiGFAP (1:1000; Chemicon) to visualize astrocytes or rat anti-mouse CD11b (1:1000, Serotec) to visualize microglia. All antibodies were diluted in a solution containing 
$10 \mathrm{mM}$ PBS with $1 \%$ sodium azide and $0.3 \%$ Triton-X. Sections were then washed with $10 \mathrm{mM}$ PBS 3 times prior to incubating with their respective secondary biotinylated antibodies, namely goat anti-mouse and goat anti-rat (1:200, Jackson Immunoresearch Laboratories), for 2-hrs at room temperature. Following 3 washes with $10 \mathrm{mM}$ of PBS, the sections were then incubated in tertiary peroxidase-conjugated streptavidin $(1: 200$, Jackson Immunoresearch Laboratories) for 2-hrs at room temperature. The antibody complexes were visualized by incubating with $\mathrm{DAB}$ for 10 minutes. Sections were mounted on glass slides, air-dried overnight, cover-slipped and stored at room temperature. Both astrocytic and microglial reactivity were qualitatively assessed using a double blind procedure. Representative photomicrographs were taken on an Olympus BX52 microscope using a $40 \mathrm{X}$ objective.

\section{General Stereotaxic Surgical procedures for Experiments 1 and 2}

All mice underwent stereotaxic surgery wherein indwelling cannulae (26-gage stainless steel) were implanted just above the SNc (bregma: anterior-posterior $-3.16 \mathrm{~mm}$, lateral $\pm 1.2 \mathrm{~mm}$, ventral $-4.0 \mathrm{~mm}$ ). Central administration of LPS or saline was achieved using a Harvard Apparatus Pico Plus syringe pump. A total of $2 \mu$ l of fluid was delivered to the SNc over a period of 5 minutes from polyethylene tubing connected to a Hamilton microliter syringe. Infusions and injections were administered between $0830 \mathrm{~h}$ and $1400 \mathrm{~h}$ to minimize the potentially confounding effects of circadian variation. It is important to note that animals were unrestrained during the infusion procedure, allowing the mice to move freely about their home cage in order to minimize stress. Also, mice had a one week convalescence period between cannulae implantation and administration of any treatment. This is an important point to address given that inflammatory processes are 
markedly affected by wounds resulting from surgical procedures and the stress associated with restraint.

Experiment 3: In vitro assessment of the effects of paraquat and GM-CSF

Primary mesencephalic neuron-glia cultures were prepared from brains of embryonic day 18 (E18) $\mathrm{C} 57 \mathrm{Bl} / 6$ mice. Whole brains were extracted aseptically and the mesencephalon was isolated. After removing the blood vessels and meninges, pooled midbrain tissue was dissociated using a papain dissociation kit (Worthington Biochemical Corp.). Briefly, $500 \mu 1$ of papain solution was added to pooled midbrain tissue and mechanically homogenized and placed in an incubator for 1 -hr at $37^{\circ} \mathrm{C}$. Cultures were maintained at $37^{\circ} \mathrm{C}$ in a humidified atmosphere of $5 \% \mathrm{CO}_{2}, 95 \%$ air. Following dissociation, the cells were triturated 5 times and centrifuged at $300 \times g$ for 5 minutes. The cells were then re-suspended in a papain/digestion inhibitor solution and counted using a hemacytometer (BrightLine; 394485). Cells were diluted in supplemented Neurobasal A media containing 2\% B27, 1\% N2, $416 \mu \mathrm{M}$ L-glutamine, $41.6 \mathrm{U} / \mathrm{ml}$ penicillin and $41.6 \mu \mathrm{g} / \mathrm{ml}$ streptomycin. Thereafter, cells were seeded in either 6-well plates $\left(25.0 \times 10^{4} / \mathrm{ml}\right)$ or 4 -well slides $(5000$ cells $/ \mathrm{ml})$ pre-coated for 1 -hr with poly- $\mathrm{D}^{-}$ lysine (20ug/ml; Sigma) and fibronectin $(20 \mu \mathrm{g} / \mu \mathrm{l}$; Sigma).

Two days following seeding, the media was replaced with fresh supplemented Neurobasal A media alone or containing GM-CSF $(250 \mathrm{ng} / \mathrm{ml})$, paraquat $(30 \mu \mathrm{M})$ or paraquat $(30 \mu \mathrm{M})+\mathrm{GM}-\mathrm{CSF}(250 \mathrm{ng} / \mathrm{ml})$, and incubated for 6-hrs. Importantly, the optimal paraquat concentration and exposure time were empirically determined by performing dose (0.3-300 $\mu \mathrm{M})$ and time (1-24 hrs) course analyses. A dose and exposure time that reliably induced $30-40 \%$ loss of $\mathrm{TH}^{+}$neurons was chosen as this degree of cell 
death paralleled that observed under in vivo conditions in the aforementioned experiments. Immediately following the drug exposure, all treated cells were replaced with fresh supplemented media and those that had been incubated with GM-CSF received fresh GM-CSF for a further 24-hrs. Thereafter, mesencephalic cultures were scraped (for western blot analysis) or fixed with 4\% PFA for 24-hrs for quantification of cell death. Hence, midbrain neurons were exposed to paraquat for 6-hrs and GM-CSF for 30-hrs in total.

\section{Immunocytochemistry}

Cells were fixed with 4\% PFA for 1 -hr at room temperature in sterile PBS. Nonspecific staining was blocked using $10 \%$ normal serum diluent (containing $0.5 \%$ Triton $\mathrm{X}-100,0.03 \%$ sodium azide in PBS) for $1-\mathrm{hr}$. The total neurons were visualized using NeuN (1:800; Chemicon) and DA neurons using TH (1:800; Chemicon) antibodies. The cultures were incubated with these primary antibodies (diluted in the blocking solution) overnight at $4^{\circ} \mathrm{C}$. The following day the cultures were washed 3 times with PBS for 10 minutes and incubated for 1-hr with secondary antibodies (1:200; Alexa 488 and Alexa 555). All images were analyzed using an up-right Nikon microscope.

\section{Western Blot}

Western blot procedures were identical to those already described for Experiment 1, with two exceptions: 1) midbrain instead of hippocampal tissue was processed, and 2) in addition to whole cell lysate fractions for BDNF, sub-cellar fractions were obtained for Bax and Bcl-2 proteins. Given the evidence that suggests GM-CSF can affect apoptotic mitochondrial proteins, it was of interest to determine any changes in pro-and antiapoptotic factors within the cytosolic and mitochondrial fractions. Specifically, the pro- 
apoptotic factor, Bax, is translocated from the cytosol to the mitochondrial membrane following activation, whereas the anti-apoptotic Bcl-2 is permanently associated with the mitochondrial membrane. To this end, subcellular fractionation was performed using a kit purchased from Calbiochem (QIA88), which separated midbrain cultures into cytosol and mitochondrial sub-fractions in order to determine Bcl-2 and Bax protein concentrations. Briefly, midbrain cell pellets were suspended in $200 \mu \mathrm{l}$ of $1 \mathrm{X}$ cytosol extraction buffer containing protease inhibitor cocktail and DTT. The cells were homogenized using a tissue grinder, centrifuged $(700 \times g$ for $10 \mathrm{~min}$ and $10000 \times g$ for $30 \mathrm{~min}$ ) to obtain the cytosolic fraction. The pellets were re-suspended in $100 \mu 1$ of mitochondrial extraction buffer containing protease inhibitors and sonicated in order to obtain the mitochondrial faction. Thereafter, samples were diluted with lysis and protease inhibitor buffer (as described earlier) up to the desired protein concentration: cytosolic fractions $(50 \mu \mathrm{g})$ and mitochondrial fraction $(50 \mu \mathrm{g})$ in $20 \mu \mathrm{L}$ and $20 \mu \mathrm{L}$ loading buffer ( $5 \%$ glycerol, $5 \% \beta$ mercaptoethanol, $3 \%$ SDS and $0.05 \%$ bromophenol blue). Finally, all samples were loaded onto an acrylamide gel, transferred to PDVF and visualized using chemiluminescence procedures that were once again identical to those of the earlier BDNF experiment. Table 1 provides a list of the antibody dilutions and gel specifics. All antibodies were obtained from Santa Cruz Biotechnology (Santa Cruz, CA) except for anti-rabbit-HRP, which was purchased from Sigma Aldrich.

Table 1.

$\begin{array}{llll}\text { Primary Antibody } & \text { Catalogue \# } & \% \text { SDS-PAGE Gel } & \text { Dilutions } \\ \text { anti-Bcl-2 HRP } & \text { sc-492 HRP } & 15 & 1: 250 \\ \text { anti- Bax HRP } & \text { sc-493 HRP } & 15 & 1: 100 \\ \text { anti-BDNF } & \text { sc-546 } & 12.5 & 1: 500 \\ \text { anti-Actin - HRP } & \text { sc-47778 HRP } & - & 1: 5000 \\ \text { anti-rabbit-HRP } & \text { A6154 } & - & 1: 2000\end{array}$


Statistical Data Analysis:

All data were analyzed by ANOVA followed by Fisher's planned comparisons where appropriate. Data were evaluated using a StatView (version 6.0) statistical software package available from the SAS Institute, Inc. For the in vitro study, protein expression was quantified by densitometry (AlphaEase FC v.3.1.2, Alpha Innotech, Co., San Leandro, CA) and, unless otherwise indicated, all results are expressed as means \pm S.E.M of at least three independent experiments.

\section{Results}

Experiment 1: Supra-nigral injection of GM-CSF or GDNF attenuated LPS-paraquat induced degeneration of SNc dopamine neurons and striatal terminals.

The ANOVAs revealed that the number of surviving $\mathrm{TH}^{+}$neurons at several levels of the SNc varied as a function of the LPS, paraquat, and growth factor (GM-CSF or GDNF) treatments $\left\{F_{7,52}=6.727, F_{7,43}=3.048 p<0.001\right.$; bregma levels -3.08 and 3.28, respectively\}. As depicted in Figure 4.1, paraquat treatment alone caused approximately $30 \%$ loss of $\mathrm{TH}^{+}$neurons across $\mathrm{SNc}$ levels. Consistent with our previous report (Mangano and Hayley, 2009), priming the SNc with LPS 2-days prior to commencing the pesticide regimen resulted in an appreciably greater loss of $\mathrm{TH}$ expressing neurons $(\sim 50 \%)$. Moreover, central administration of either GM-CSF or GDNF completely prevented the neuronal loss induced by paraquat alone or following LPS priming. Indeed, post-hoc statistical analyses confirmed that paraquat and LPS + paraquat administration significantly reduced the survival of $\mathrm{TH}^{+}$neurons and individual infusion of either GM-CSF or GDNF prevented these neurodegenerative consequences ( $p$ $<0.01)$

To determine whether the loss of $\mathrm{TH}^{+}$neurons reflected a genuine neurodegenerative effect or simply a phenotypic suppression, all SNc sections were 
counterstained with cresyl violet and the total number of surviving neurons was quantified across bregma levels for each of the treatment groups. Not surprisingly, the ANOVA revealed a significant difference in the total number of cresyl violet stained neurons as a function of the treatments $\left\{F_{7,52}=5.104, F_{7,43}=3.048 p<0.001\right.$; bregma levels -3.08 and -3.28 , respectively . Specifically, in a manner identical to that observed for $\mathrm{TH}^{+}$neurons, paraquat reduced the total number of SNc neurons and once again, this effect was augmented by LPS priming and was absent in mice that received the GM-CSF or GDNF treatments ( $p<0.01$; data not shown). However, this effect appeared to be restricted to the $\mathrm{TH}^{+}$neurons, as the number of $\mathrm{TH}^{-}$cresyl violet stained neurons were not significantly affected by any of the treatments ( $p>0.05$; data not shown). Thus, it appears that there was a genuine DA neuronal loss induced by the toxins and that the trophic cytokines had protective actions.

Insert Figure 4.1 about here

As was the case for the SNc, the LPS, paraquat and growth factor treatments altered $\mathrm{TH}^{+}$immunoreactivity within the striatum $\left\{F_{7,33}=32.195 p<0.001\right\}$. Specifically, as determined using densitometry, mice exposed to paraquat alone or primed with LPS displayed reduced striatal $\mathrm{TH}^{+}$staining $(p<0.05)$; and, once again, infusion of either GM-CSF of GDNF prevented this effect, see Figure 4.2.

Insert Figure 4.2 about here 
Figure 4.1 Central administration of GM-CSF and GDNF protected SNc neurons. Dopaminergic neuron loss was assessed using TH (1:1000) antibody staining combined with cresyl violet counter-stain through several levels of the SNc. The top representative photomicrographs illustrate the degree of dopaminergic neuron loss induced by LPS and paraquat, as well as the effects of GDNF and GM-CSF. Priming the SNc with a single supra-nigral injection of LPS $(0.01 \mu \mathrm{g} / 2 \mu \mathrm{l}) 2$ days prior to beginning the paraquat regimen $\left(10 \mathrm{mg} / \mathrm{kg} ; 3\right.$ per week for 3-weeks) (LPS-Paraquat) enhanced the $\mathrm{TH}^{+}$cell loss, relative to paraquat treatment alone $\left({ }^{*} \mathrm{p}<0.05\right)$. However, a single supra-nigral injection of GDNF $(1 \mu \mathrm{g} / 2 \mu \mathrm{l})$ or GM-CSF $(10 \mathrm{ng} / 2 \mu \mathrm{l})$ once per week during the paraquat regimen prevented the SNc neuron loss induced by paraquat alone and with the addition of LPS. Data are expressed as mean $\pm \mathrm{SEM} ; \mathrm{n}=8-10$. 


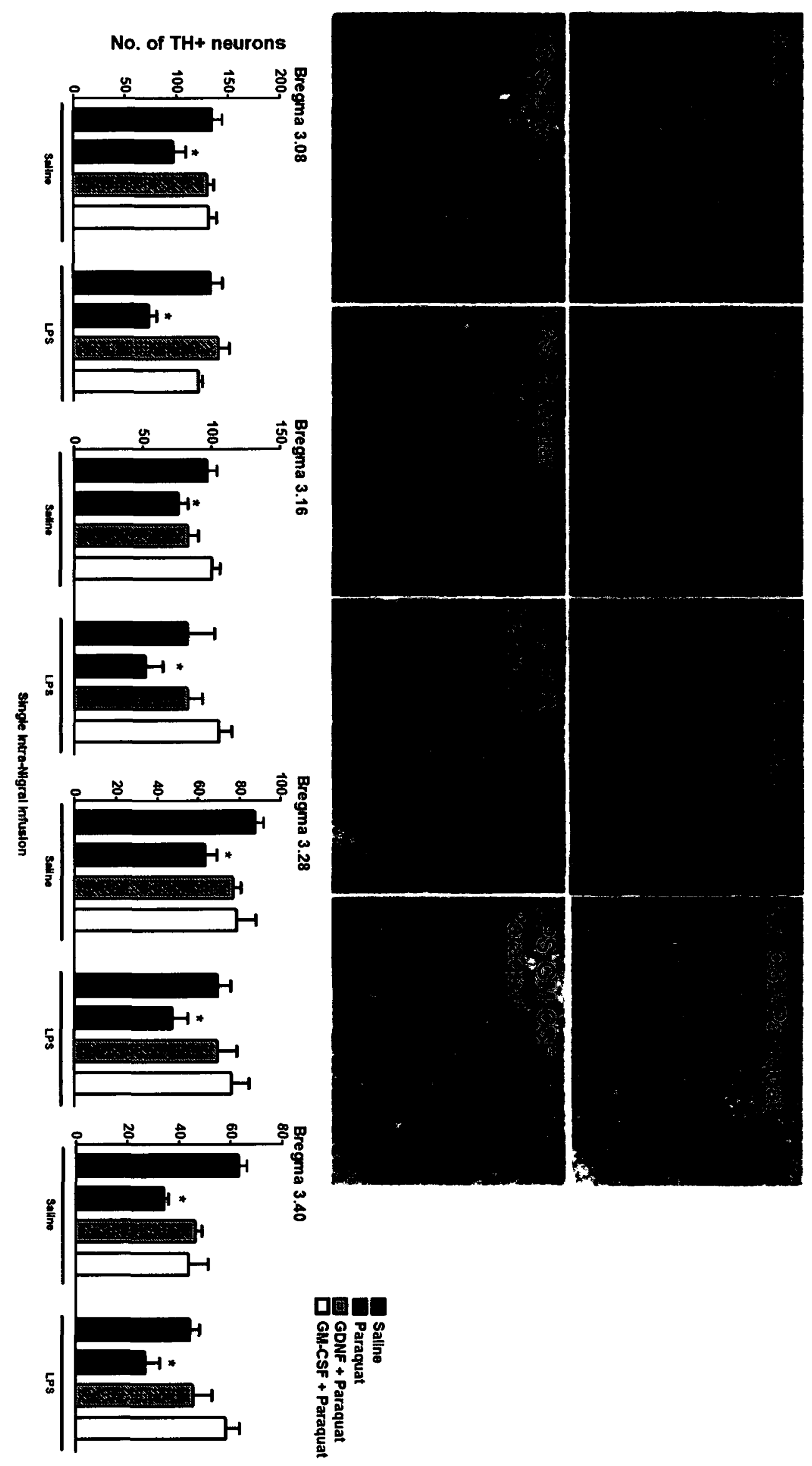


Figure 4.2 Central administration of GM-CSF and GDNF attenuated the impact of LPS and paraquat upon striatal dopaminergic terminals.

Striatal DA fiber loss was determined by analyzing pixel intensity of sections stained with TH (1:1000). The representative photomicrographs illustrate that paraquat with or without LPS priming induced a reduction of $\mathrm{TH}^{+}$fiber staining. Indeed, mice exposed to paraquat $(10 \mathrm{mg} / \mathrm{kg} ; 3$ per week for 3-weeks) alone or following priming 2-days earlier with LPS $(0.01 \mu \mathrm{g} / 2 \mu \mathrm{l})$ displayed approximately $35 \%$ loss of striatal terminals $\left({ }^{*} \mathrm{p}<\right.$ $0.05)$. A single supra-nigral injection of GDNF $(1 \mu \mathrm{g} / 2 \mu \mathrm{l})$ or GM-CSF $(10 \mathrm{ng} / 2 \mu \mathrm{l})$ once per week during the paraquat regimen totally prevented this loss of $\mathrm{TH}^{+}$fiber immunoreactivity. Data are expressed as mean $\pm \mathrm{SEM} ; \mathrm{n}=8-10$ 
Proinflammatory cytokines and Parkinson's disease, 150
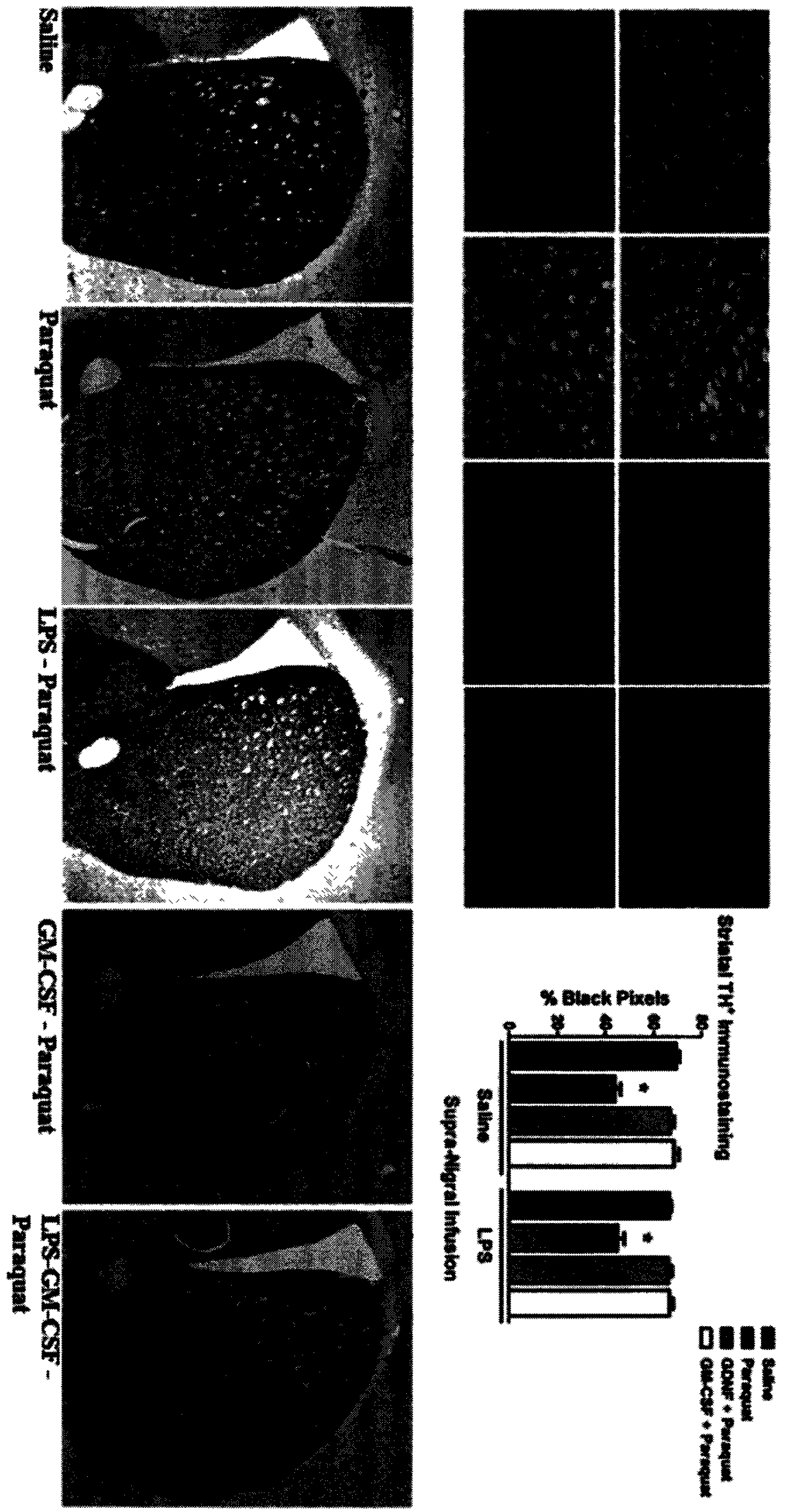
Supra-nigral injection of GM-CSF influenced LPS and paraquat induced glial changes in the $S N c$.

LPS and paraquat induced changes in microglial morphology that paralleled the loss of $\mathrm{TH}^{+}$neurons within the SNc. As previously observed (Mangano and Hayley, 2009), paraquat administered alone or in LPS primed mice enhanced microglial reactivity (as indicated by morphological changes and increased CD11b density) within the SNc. Specifically, paraquat induced a modest elevation of $\mathrm{CD} 11 \mathrm{~b}^{+}$staining in comparison with mice treated only with saline, and prior LPS infusion further enhanced this effect. The majority of "activated" microglia displayed an intermediate level of reactivity characterized by shortened, thick dendritic processes, while a few cells, particularly in response to LPS and paraquat treatment, appeared to be clumped together with more compact soma characteristic of a reactive state, Figure 4.3. Surprisingly, a single supranigral injection of GDNF at the beginning of each week of the paraquat regimen did not appreciably influence the impact of paraquat or LPS upon CD11 $\mathrm{b}^{+}$immunoreactivity. However, the weekly GM-CSF infusions did cause a change in the state of microglial activation, wherein the trophic cytokine greatly attenuated the morphological changes of microglia that were provoked by paraquat and LPS, see Figure 4.3.

In contrast to the augmented microglial response (at least in terms of morphology), astrocytic expression within the $\mathrm{SNc}$ (as indicated by $\mathrm{GFAP}^{+}$ immunoreactivity) was reduced in mice exposed to paraquat, see Figure 4.3. Moreover, as we previously reported (Mangano and Hayley, 2009), this GFAP reduction was greatly augmented in mice that had been primed with LPS prior to exposure to the pesticide. Importantly, a single infusion of either of the growth factors, GDNF or GM-CSF, once 
per week throughout the paraquat regimen normalized the LPS/paraquat induced reduction of GFAP staining, see Figure 4.3.

Insert Figure 4.3 about here

Supra-nigral infusion of GM-CSF modified the paraquat induced reduction of hippocampal BDNF.

As already alluded to, disturbances of neurotrophin-mediated plasticity might be important not only for the cognitive deficits observed in $\mathrm{PD}$, but also the other frequent co-morbid psychiatric symptoms, such as anxiety and depression (Litteljohn et al., 2009). To this end, it is important that the paraquat and GM-CSF treatments provoked alterations of hippocampal BDNF $\left\{F_{2,10}=8.597 p<0.01\right\}$, see Figure 4.4. Specifically, BDNF was reduced within the hippocampus following paraquat administration, relative to vehicle treatment $(p<0.05)$; however, GM-CSF infusion attenuated this effect, such that the growth factor did not differ from controls. Owing to tissue limitations, the Western blot analyses were restricted to only the vehicle, paraquat and GM-CSF + paraquat treatment groups.

Insert Figure 4.4 about here

Experiment 2: Systemic administration of GM-CSF had neuroprotective consequences. As in Experiments 1, the ANOVA revealed a significant difference between the treatment groups in terms of the number of surviving $\mathrm{TH}^{+}$neurons within the $\mathrm{SNc}$ 
Figure 4.3 Central GDNF and GM-CSF modulated the impact of LPS and paraquat upon $S N c$ astrocytes and microglia.

The representative photomicrographs depict astrocyte $\left(\mathrm{GFAP}^{+} ; 1: 1000\right)$ and microglia $\left(\mathrm{CD} 11 \mathrm{~b}^{+} ; 1: 1000\right)$ immunostaining within the SNc of mice that received supra-nigral saline or LPS infusion together with systemic paraquat treatment, in presence or absence of GDNF $(1 \mu \mathrm{g} / 2 \mu \mathrm{l})$ or GM-CSF $(10 \mathrm{ng} / 2 \mu \mathrm{l})$. The top panels show that GFAP immunoreactivity was modestly diminished by paraquat alone, whereas a more robust reduction was apparent with the combined exposure to LPS and paraquat, relative to saline treatment. Once again, the GDNF and GM-CSF infusions appeared to reverse the effects of LPS and paraquat and in fact, GM-CSF treatment appeared to increase GFAP staining slightly above that of saline treated controls. As shown in the bottom panels, paraquat elevated $\mathrm{CD} 1 \mathrm{lb}^{+}$immunostaining within the $\mathrm{SNc}$ and this effects was more pronounced in the LPS primed mice. Interestingly, although GM-CSF infusion blunted the impact of LPS and paraquat (in terms of CD1lb+ staining intensity), GDNF had no such influence on the microglial marker. 
$\stackrel{8}{3}$

\section{5 \\ 足}
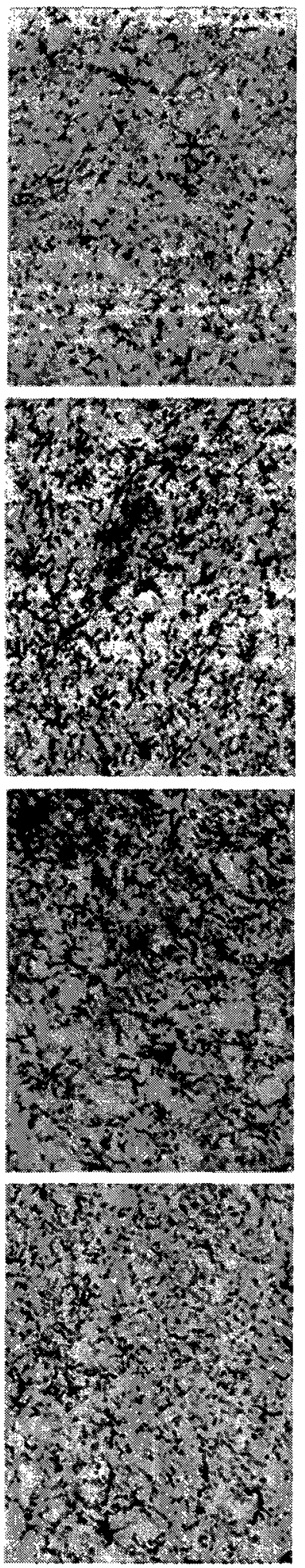

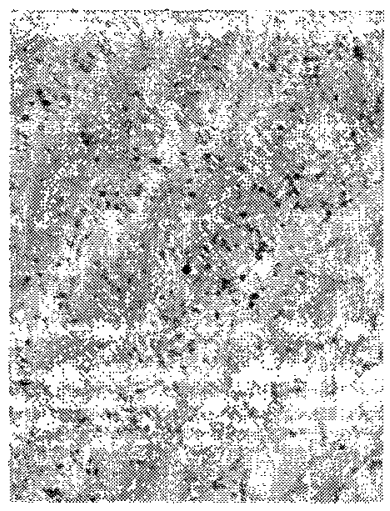

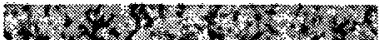

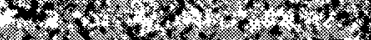

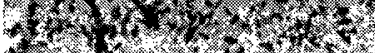

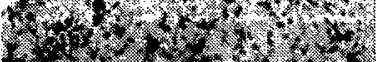

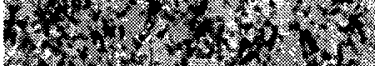

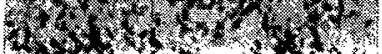

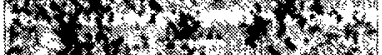

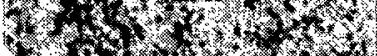
W0 30 , wrot 62 30 s
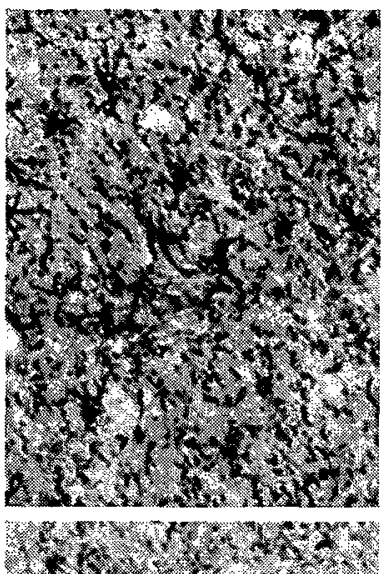

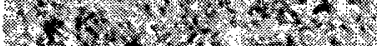

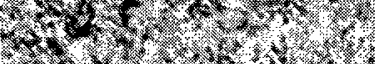

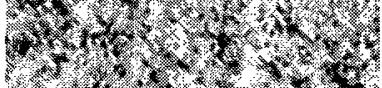

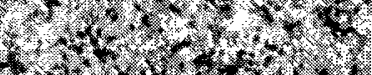

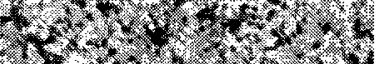

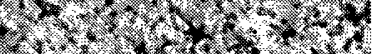

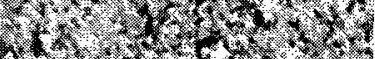

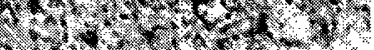

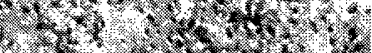

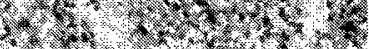

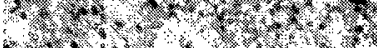
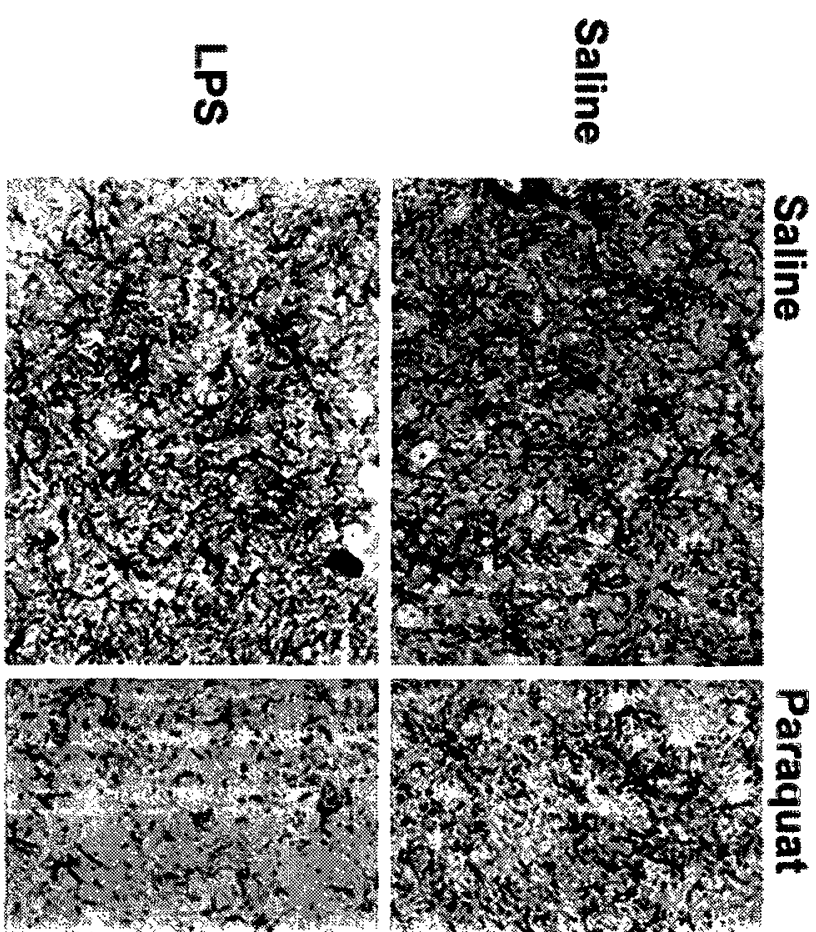

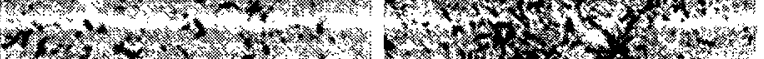

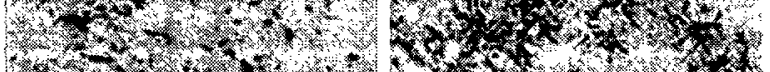

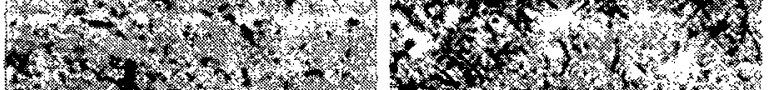

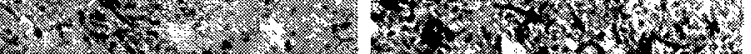
(2)
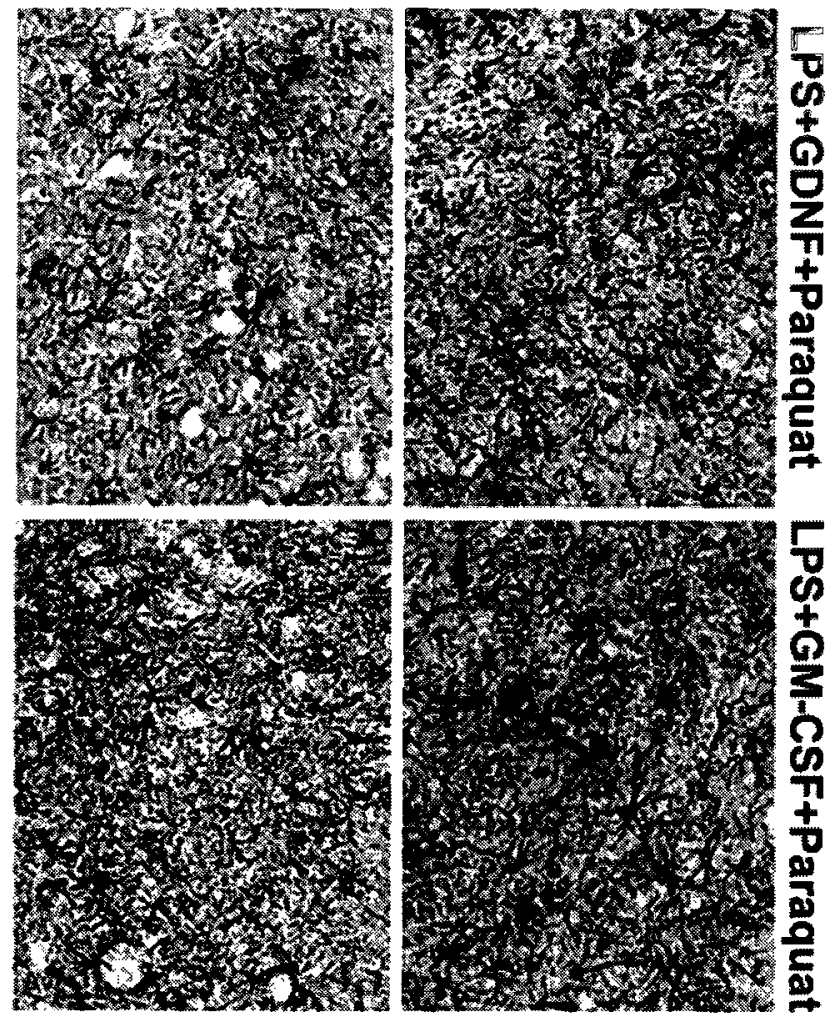


\section{Hippocampus: BDNF}
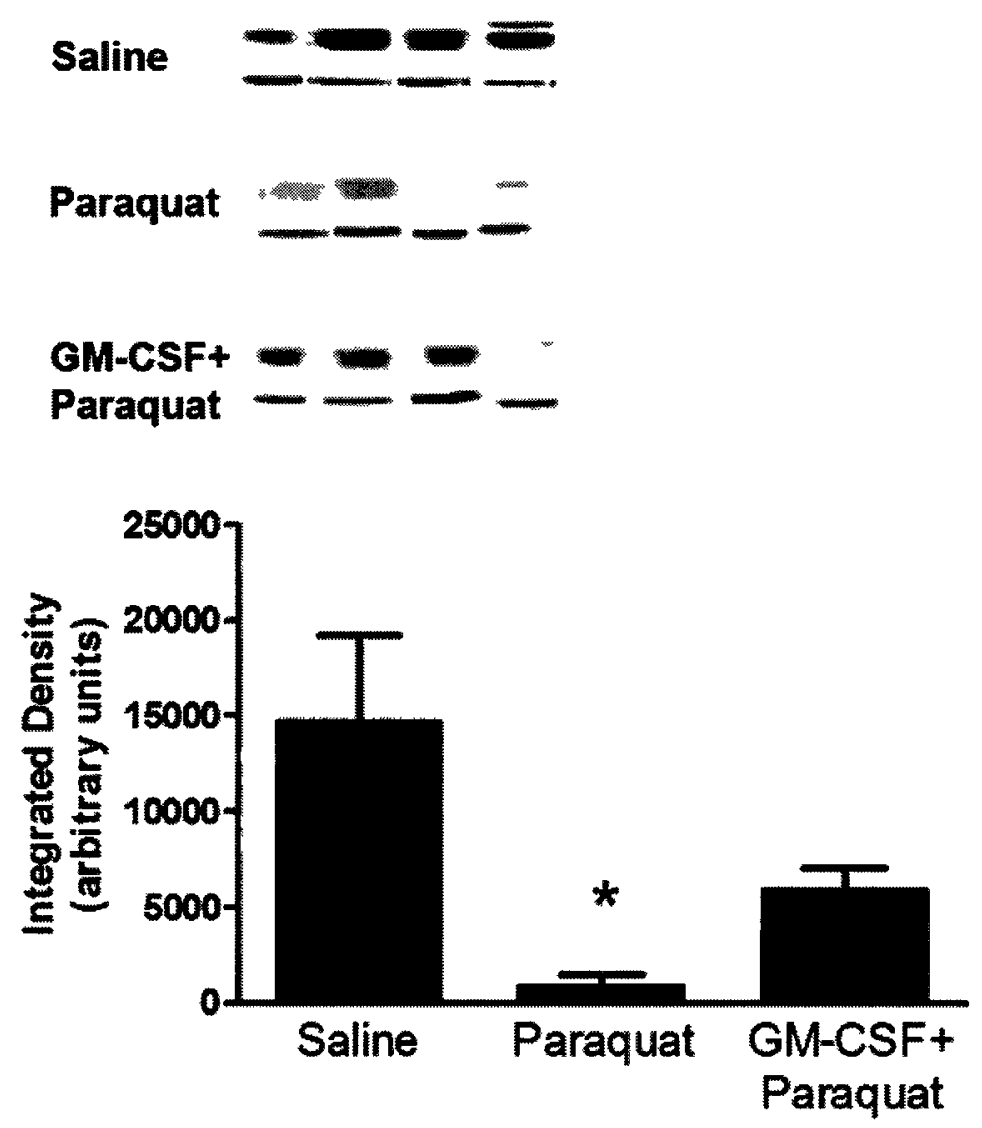

Figure 4.4 Central administration of GM-CSF influenced the effects of paraquat on hippocampal BDNF expression.

The Western blots revealed that paraquat promoted a marked reduction of hippocampal BDNF protein. Four representative bands are shown from the three treatment groups. Clearly, paraquat caused a reduction of BDNF in each of the animals exposed, whereas the addition of GM-CSF $(10 \mathrm{ng} / 2 \mu \mathrm{l})$ infusion generally attenuated this effect (at least in 3 of the 4 animals). The bar graph on the bottom confirmed that these effects were statistically significant ( ${ }^{*} p<0.05$, relative to saline treated). All integrated density scores were normalized against $b$-actin to control for any variations in loading. Data are expressed as mean $\pm \mathrm{SEM} ; \mathrm{n}=4$. 
$\left\{F_{3,12}=79.701 p<0.0001\right\}$. The follow-up comparisons indicated that chronic paraquat exposure significantly reduced the number of $\mathrm{TH}^{+} \mathrm{SNc}$ neurons $(\sim 30 \%$ reduction) relative to vehicle-treated mice $(p<0.01)$, and this effect was further amplified when animals were pre-treated with LPS before exposure to the pesticide ( $>40 \%$ reduction). Paralleling the results of supra-nigral infusion in Experiment 1, i.p. GM-CSF injection appeared to attenuated the impact of LPS and paraquat, such that the number of surviving SNc $\mathrm{TH}^{+}$neurons did not significantly differ from those that received vehicle alone, see Figure 4.5. Once again, the impact of paraquat appeared to be restricted to DA neurons; indeed, although the total number of cresyl violet stained neurons was reduced, no such reduction was observed for specific $\mathrm{TH}^{-}$cresyl violet neurons (data not shown).

Again paralleling the earlier findings, the LPS, paraquat and GM-CSF treatments affected $\mathrm{TH}^{+}$immunoreactivity within the striatum $\left\{F_{3,14}=31.592 p<0.0001\right\}$. As depicted in Figure 4.5, mice exposed to paraquat alone or primed with LPS displayed reduced striatal $\mathrm{TH}^{+}$immunostaining $(p<0.05)$. Moreover, systemically applied GMCSF appeared to, once again, preserve striatal DA nerve terminals following LPS/paraquat exposure.

Insert Figure 4.5 about here

As was the case for central infusion of GM-CSF, systemic administration of the trophic cytokine appeared to blunt the $\mathrm{CD} 11 \mathrm{~b}^{+}$changes induced by LPS and paraquat (Figure 4.6). Specifically, both the LPS and chronic paraquat treatments increased 
Figure 4.5 Systemic GM-CSF treatment protected nigrostriatal neurons (both SNc soma and striatal terminals) from LPS and paraquat induced dopaminergic cell death.

The loss of dopamine (DA) neurons was determined using stereological cell counts of $\mathrm{TH}^{+}(1: 1000)$ neurons through the SNc. The top left representative photomicrographs illustrate the degree of DA cell loss induced by LPS and paraquat, as well as the protective effects of GM-CSF. Once again, priming the SNc with a single supra-nigral injection LPS $(0.01 \mu \mathrm{g} / 2 \mu \mathrm{l}) 2$ days prior to commencing the paraquat regimen (10 $\mathrm{mg} / \mathrm{kg}$; $3 \mathrm{x}$ per week for 3-weeks) enhanced the degree of neuronal loss from approximately $25 \%$ (paraquat alone) to $40 \%(* \mathrm{p}<0.05)$. Moreover, as shown in the top right photomicrographs, mice exposed to the paraquat regimen displayed a significant loss of striatal fibers $(* p<0.05)$, however, LPS priming did not further augment this effect. The bottom bar graphs show the quantification of SNc soma and striatal terminal loss and the apparent protective effects of systemic (i.p.) administration of GM-CSF (2 $\mu \mathrm{g})$. Data are expressed as mean $\pm \mathrm{SEM} ; \mathrm{n}=5$. 
SNC
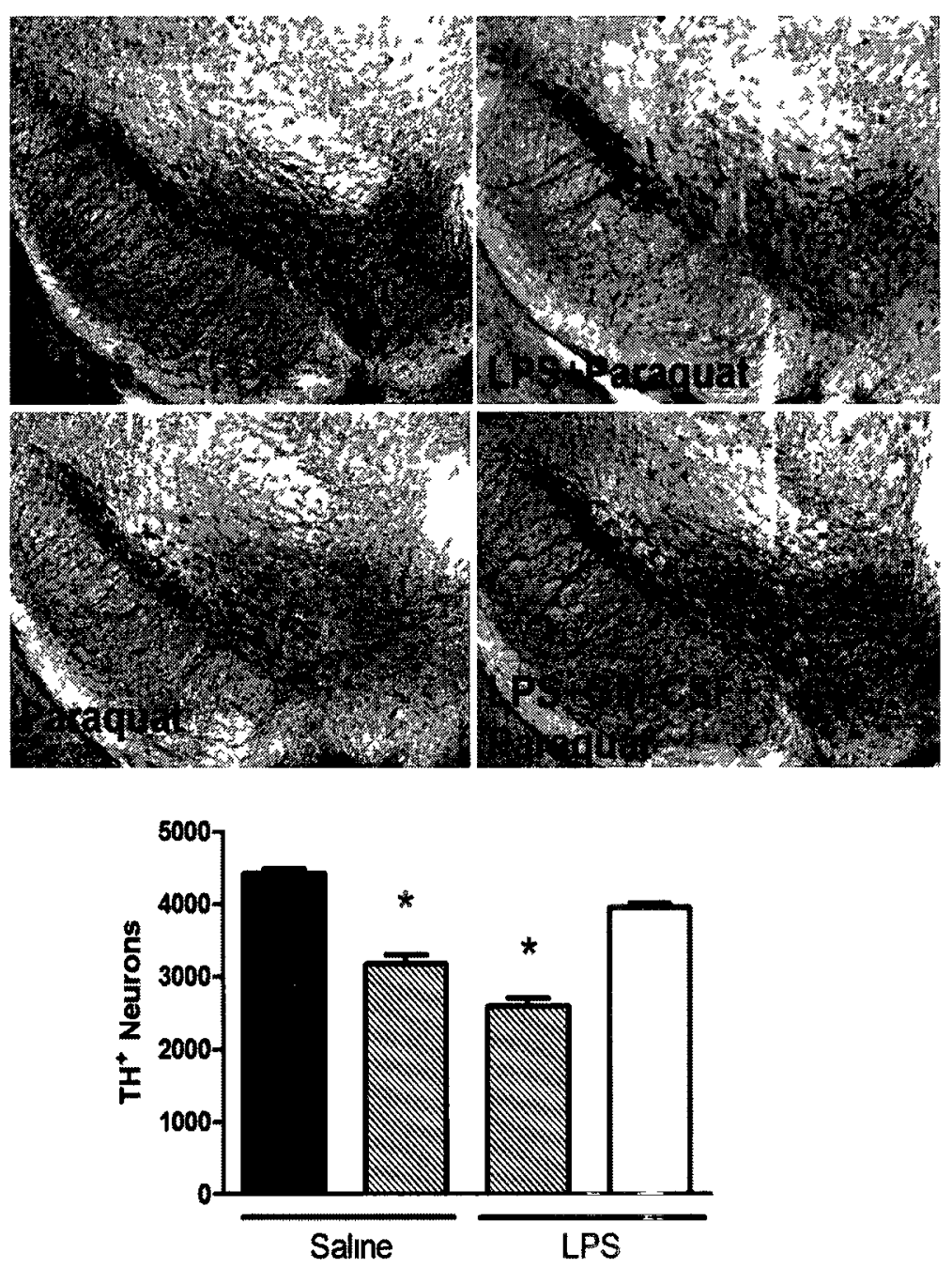

\section{Striatum}
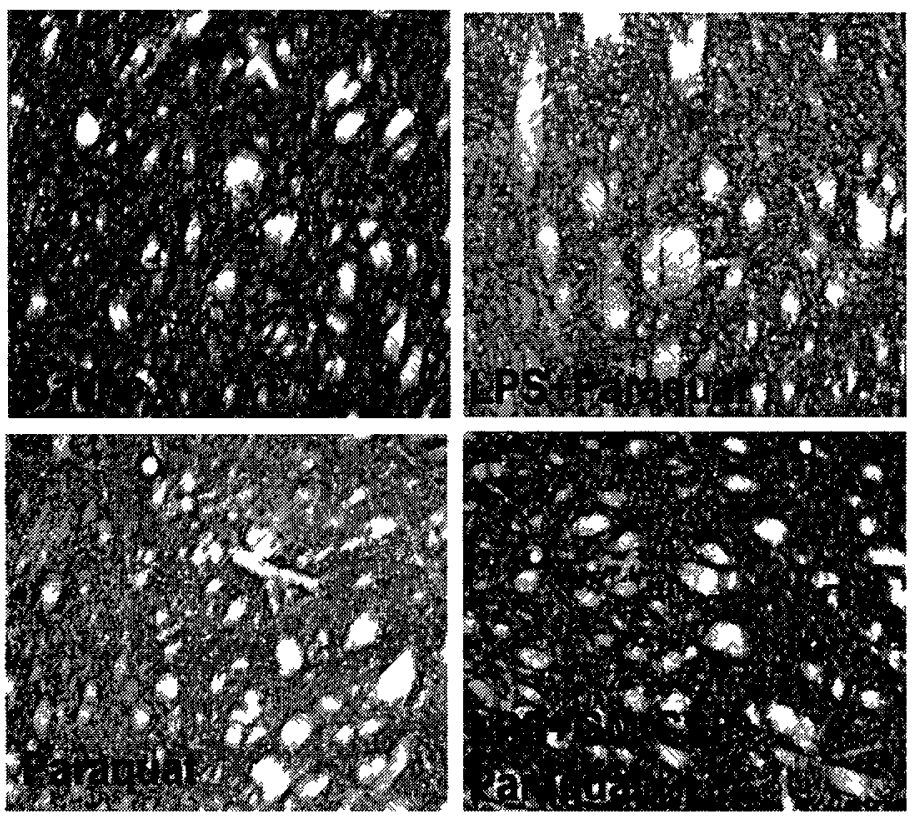

Saline

GM-CSF + Paraquat

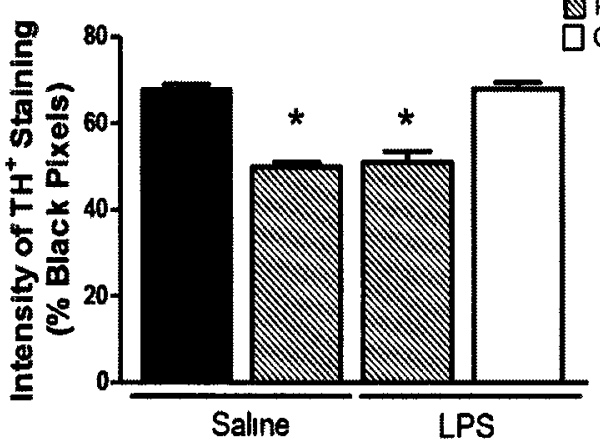




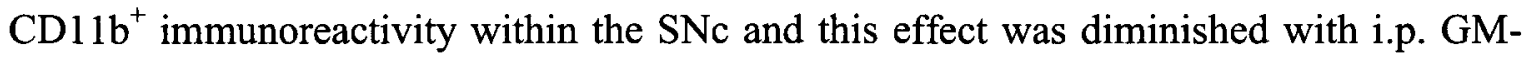
SCF administration. Once again, as was observed with central GM-CSF infusion, systemic exposure to the cytokine augmented GFAP immunoreactivity, whereas LPS and paraquat appeared to moderately suppress the astrocytic marker.

\section{Insert Figure 4.6 about here}

Experiment 3: GM-CSF neuroprotection was associated with normalized BDNF but not Bcl-2 or Bax levels

To evaluate the mechanism utilized by GM-CSF to protect SNc DA neurons from paraquat toxicity, primary mesencephalic neuron-glia mixed cultures were assessed following paraquat exposure. A dose response curve and time dependent evaluation of paraquat toxicity revealed the dose that provoked a degree of cell death similar to that observed in vivo (data not shown). Specifically, the dose (0.3-300 $\mu \mathrm{M})$ and time (1-24 hrs) course analyses revealed that exposure to $30 \mu \mathrm{M}$ of paraquat for 6 -hrs provided optimal conditions, reliably inducing $30-40 \%$ loss of $\mathrm{TH}^{+}$neurons. Indeed, further experiments conducted using these parameters revealed significant differences in the number of surviving $\mathrm{TH}^{+}$neurons of the midbrain cultures as a function of pesticide exposure $\left\{F_{3,16}=13.048 p<0.0001\right\}$. As shown in Figure 4.7, paraquat provoked a significant loss of midbrain $\mathrm{TH}^{+}$neurons $(p<0.01)$; however, again paralleling the in vivo findings, this effect was completely prevented by co-treatment with GM-CSF (250 $\mathrm{ng} / \mathrm{ml})$.

Given that GM-CSF has been reported to impart protective consequences through its effects upon trophic and apoptotic pathways (Huang et al., 2007), the trophic factor, 
Figure 4.6 Systemic GM-CSF administration modulated the effects of LPS and paraquat upon $S N c$ astrocytes and microglia.

The top photomicrographs revealed that paraquat and LPS + paraquat robustly induced

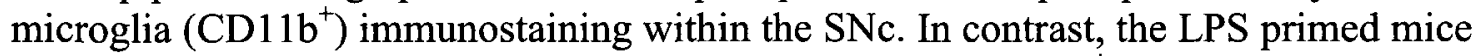
that received paraquat displayed a reduction of astrocyte $\left(\mathrm{GFAP}^{+}\right)$staining, whereas paraquat alone had little effect. Paralleling the aforementioned effects apparent with central GM-CSF administration, systemic injection of the trophic cytokine blunted the impact of LPS and paraquat upon the glial populations. 


\section{署高}
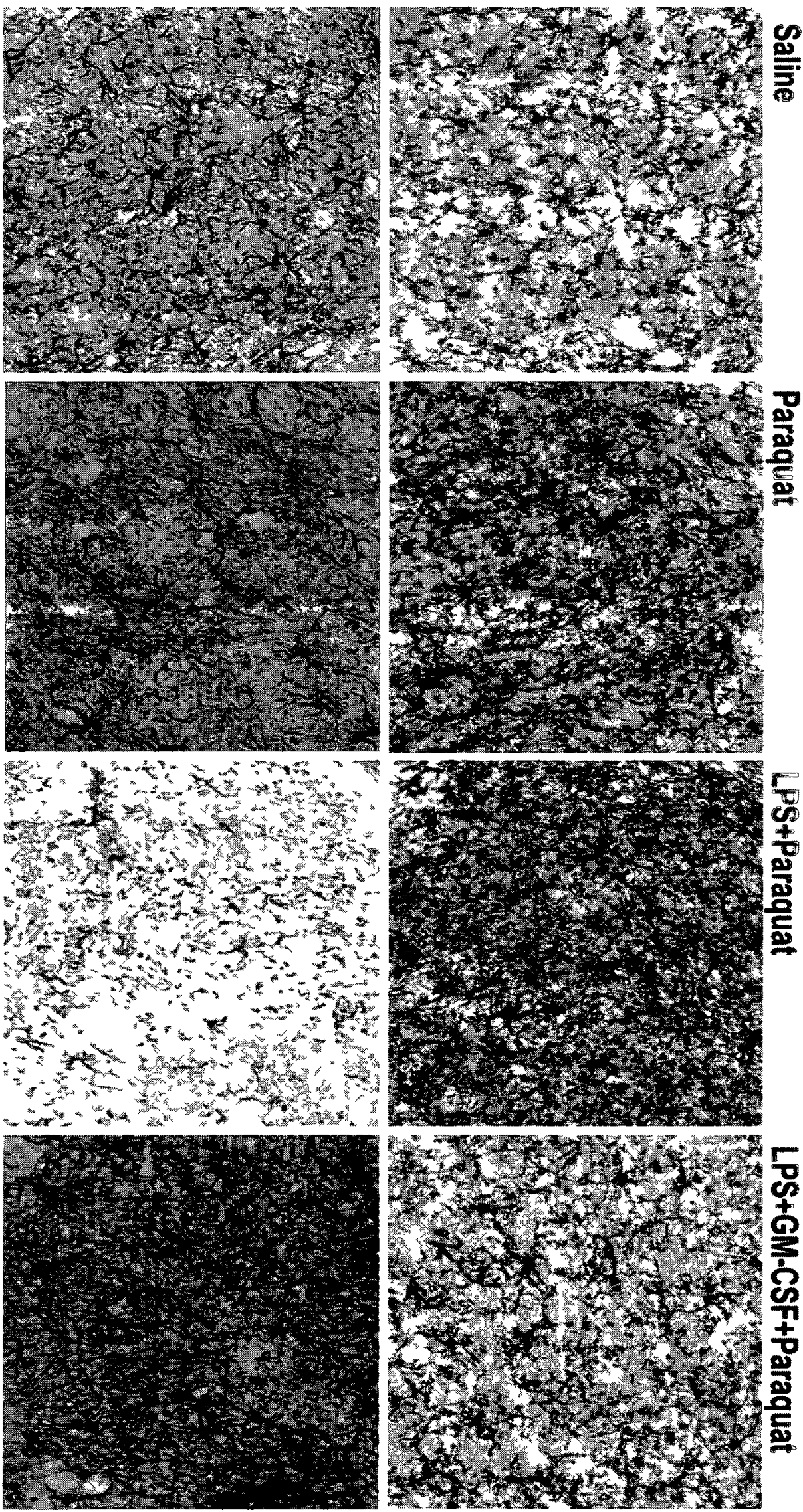
$\mathrm{BDNF}$, and the pro- and anti-apoptotic factors, Bax and Bcl-2, respectively, were assessed using Western blot. To this end, the ANOVAs revealed significant differences between the treatment groups for BDNF and Bcl-2 (but not Bax) protein levels within the midbrain cultures $\left\{F_{2,4}=6.412, F_{2,9}=3.881\right.$, respectively, $\left.p<0.05\right\}$. As depicted in Figure 4.7 and confirmed by the follow up comparisons, paraquat significantly reduced midbrain levels of BDNF and Bcl-2 $(p<0.05)$. Co-treatment with GM-CSF restored BDNF protein expression within the midbrain cultures; however, the growth factor did not appear to affect Bcl-2 protein expression.

Insert Figure 4.7 about here

\section{Discussion}

Inflammatory and oxidative processes, together with reduced trophic support, are widely considered to be essential players in the pathological processes of PD (Bossers et al., 2009; Chauhan et al., 2001; Guerini et al., 2009; Howells et al., 2000; Masaki et al., 2003). Indeed, a plethora of evidence suggests that an augmented microglial response, possibly driven by pro-inflammatory cytokines such as IFN- $\gamma$ and TNF- $\alpha$ contributes to the progression of PD (Hirsch and Hunot, 2009; Whitton, 2007). In this regard, microglial release of oxidative species, coupled with enhanced inflammatory enzyme activity (e.g., COX-2) and induction of various MAP kinase pathways represent proximal mediators of DA neuronal pathology (Gao et al., 2003b; Hunot et al., 2004). In contrast to the more pro-inflammatory role of microglia, astrocytes have more frequently been associated with a protective, buffering capacity and the release of trophic factors 
Figure 4.7 GM-CSF restored hippocampal BDNF expression in midbrain cultures exposed to paraquat.

As depicted by the above representative blots and confirmed by densitometry paraquat $(30 \mu \mathrm{M})$ exposure for 6-hrs induced a significant reduction in BDNF and Bcl-2 levels in primary midbrain tissue $\left({ }^{*} \mathrm{p}<0.05\right)$. When applied concomitant with paraquat, GM-CSF $(250 \mathrm{ng} / \mathrm{ml})$ partially prevented the reduction of BDNF but had no effect on Bcl-2 levels within the midbrain. Paralleling the aforementioned in vivo data, paraquat exposure reduced the number of $\mathrm{TH}^{+}$neurons within the midbrain cultures $(\mathrm{p}<0.05)$ and once again, GM-CSF reversed this effect (bottom bar graph). Data are expressed as mean \pm $\mathrm{SEM} ; \mathrm{n}=2-3(\mathrm{BDNF})$ and $\mathrm{n}=4-5(\mathrm{Bcl}-2)$. 


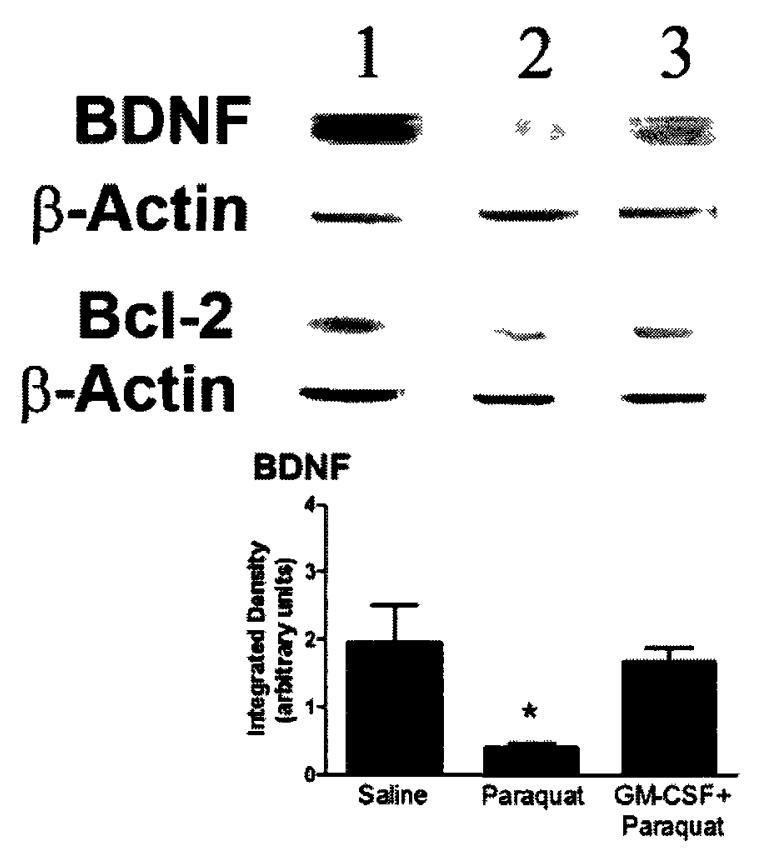

\section{Legend \\ 1: Saline \\ 2: Paraquat}

3: GM-CSF + Paraqua

Primary Mesencephalic cultures
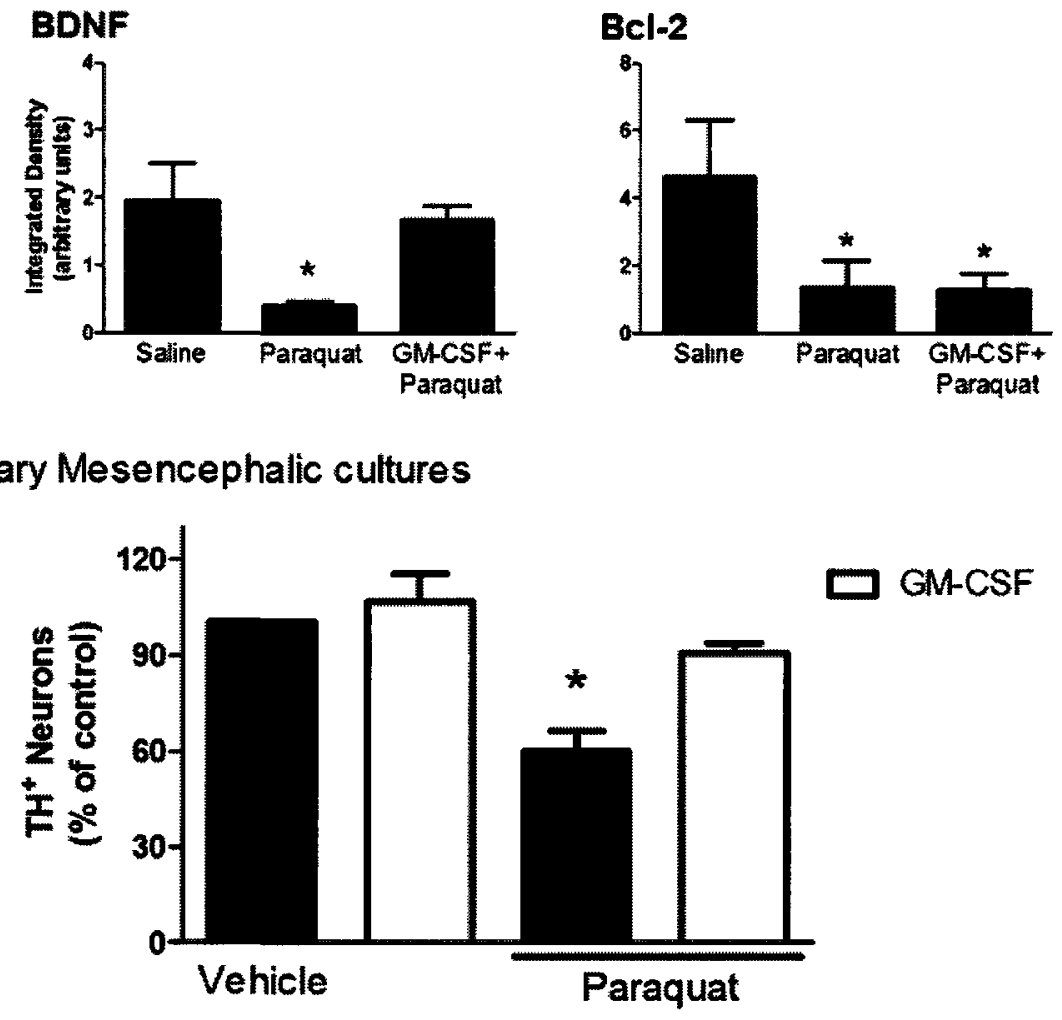
(Chen et al., 2006). At the same time, several reports detected reductions of anti-oxidants, particularly glutathione, as well as the trophic factor, BDNF, in PD brains (Fitzmaurice et al., 2003; Murer et al., 2001). Whatever the case, increasing attention has been devoted to utilizing novel means of interfering with the pro-death process in animal models of PD. To this end, we report that both central and peripheral administration of the trophic cytokine, GM-CSF, protected SNc DA neurons from LPS and paraquat toxicity, and modulated BDNF, microglia and astrocyte responses to these toxins.

Neurotrophic factors are essential for the maintenance and survival of neuronal populations. Consequently, both BDNF and GDNF have been extensively studied as potential therapeutic agents for PD patients (Peterson and Nutt, 2008). BDNF is normally present in relatively high concentrations within SNc DA neurons and is responsible for supplying nutritive support, as well as dampening inflammatory and oxidative pathways (Baquet et al., 2005; Murer et al., 2001). The trophic factor can also promote the survival and differentiation of mesencephalic DA neurons, as well as protect against MPTP and 6OHDA insults (Hung and Lee, 1996; Shults et al., 1995). In agreement with the finding that BDNF mRNA levels are reduced within the SNc of post-mortem PD brain (Mogi et al., 1999; Salehi and Mashayekhi, 2009) we presently report that paraquat diminished midbrain BDNF levels in mice. Moreover, in vitro application of GM-CSF reversed this effect, just as it prevented the loss of cultured midbrain $\mathrm{TH}^{+}$neurons induced by paraquat. These findings are consistent with reports showing that GM-CSF can augment BDNF to combat spinal cord injury (Bouhy et al., 2006; Hayashi et al., 2009); and, taken together, suggest that BDNF could be contributing to some of the neuroprotective actions of GM-CSF in response to diverse injury-inducing stimuli. 
In addition to BDNF, GDNF acts on dopaminergic neurons to enhance their morphological differentiation and survival (Lin et al., 1993), which has led to much excitement regarding the potential of GDNF as a therapeutic agent for PD. Indeed, numerous rodent and primate studies reported that GDNF attenuated the neurodegenerative effects of MPTP and further boosted DA functioning of the existing neurons (Gash et al., 1996; Kearns and Gash, 1995; Tomac et al., 1995). Importantly, the GDNF-induced restoration of DA functioning was apparent even months after trophic support was terminated (Grondin et al., 2002; Kirik et al., 2004; Maswood et al., 2002). Despite the overwhelming evidence supporting the benefits of GDNF, it has largely failed in human clinical trials due to complications arising from the fact that GDNF is unable to cross the BBB. Indeed, intracerebroventricular injection of GDNF was reported to cause problems related to (limited) diffusion into the brain parenchyma and the manifestation of debilitating side-effects (Kordower et al., 1999; Nutt et al., 2003).

To circumvent the fact that trophic factors generally do not cross the BBB, new techniques are currently being developed to facilitate central penetration (JuilleratJeanneret and Schmitt, 2007; Pardridge, 2002); these include encapsulation or breaking GDNF into small bioactive fragments (Peleshok and Saragovi, 2006). However, finding novel neurotrophic factors that easily cross the BBB (and thus avoid such complications entirely) may provide a new class of therapeutic agents for PD. In this regard, GM-CSF is one of three cytokines belonging to a family of colony stimulating factors that have trophic effects and are capable of crossing the BBB and accumulating in the brain parenchyma at reasonable levels (Franzen et al., 2004; McLay et al., 1997; Thomson and Lotze, 2003). The exact mechanism by which GM-CSF permeates the BBB is presently 
unclear; however, based on experiments which tagged GM-CSF prior to systemic administration to rodents, it is clear that this cytokine can, indeed, gain entry into the brain (McLay et al., 1997).

GM-CSF has well documented clinical efficacy in the periphery, including its use as an immune restorative agent in certain cancer treatments (owing to its ability to stimulate leukocyte production); however, minimal attention has been devoted to elucidating the trophic cytokine's potential as a neurotrophic agent. Albeit, a few reports have indicated that GM-CSF had neurorestorative functions in the spinal cord and even assisted in cerebral ischemic recovery (Ha et al., 2005; Hayashi et al., 2009; Nakagawa et al., 2006; Schabitz et al., 2008). There is also some evidence to suggest that GM-CSF can promote the release of BDNF from microglia (Bouhy et al., 2006; Hayashi et al., 2009). In addition, GM-CSF has been shown to down-regulate the IFN- $\gamma$ mediated induction of MHC-II expression on microglia (Hayashi et al., 1993). This is important since IFN- $\gamma$ was recently shown to play a primary role in the degeneration of DA neurons following paraquat or MPTP exposure (Mount et al., 2007). These findings, in combination with the fact that GM-CSF can promote a phenotypic shift in microglial functioning towards a more supportive and less inflammatory role (Bouhy et al., 2006; Re et al., 2002), prompted us to hypothesize that GM-CSF might protect against DA acting toxins by modulating the inflammatory balance between microglial and astrocytic cells.

Consistent with reports showing that GM-CSF might affect neuronal survival by inducing microglia to adopt a dendritic cell-like morphology (Reddy et al., 2009), exposure to the growth factor in the current study appreciably diminished the impact of LPS and paraquat upon microglia (as indicated by staining intensity). However, the well 
established neurotrophin, GDNF, did not appreciably affect microglia staining (at least according to $\mathrm{CD} 11 \mathrm{~b}^{+}$cell morphology and proliferation), despite the fact that the growth factor prevented DA neuron loss to a degree comparable to that of GM-CSF. Thus, the two trophic factors appear to be promoting DA neuronal survival through different processes, at least with regards to microglial involvement.

In contrast to the heightened microglia response induced by LPS and paraquat, these insults caused a significant reduction in the SNc astrocytic response (as indicated by $\mathrm{GFAP}^{+}$immunostaining). Interestingly, both GM-CSF and GDNF reversed the astrocytic reduction induced by the toxins. This is particularly important given that an astrocyte reduction would likely result in diminished availability of trophic support (e.g., BDNF and GDNF release) and reduced glutamate buffering capabilities (Malipiero et al., 1990). Although GM-CSF receptors are certainly expressed on astrocytes (Guillemin et al., 1996; Malipiero et al., 1990), they have also been found on neurons throughout the brain, including DA neurons of the SNc (Kim et al., 2009a). Hence, while GM-CSF might conceivably be promoting neuronal survival through its anti-inflammatory-like effects upon microglia and the restoration of astrocyte functioning, it is also possible that these glial changes are simply secondary to the direct impact of the trophic cytokine upon DA neurons (or other yet to be identified processes involved in the neurodegenerative response).

Although GM-CSF's neuroprotective properties likely stem, in part, from its ability to affect glial activity and BDNF levels, the capacity of the cytokine to promote anti-apoptotic signalling might also be important (Schabitz et al., 2008). Indeed, a recent study found that GM-CSF promoted Bcl-2 expression following MPTP exposure (Kim et 
al., 2009a). However, in the present investigation, the growth factor did not influence Bcl-2 expression in primary mesencephalic cultures. This discrepancy may stem from the fact that the previous study assessed Bcl-2 within PC12 cells (Kim et al., 2009a), whereas we presently assessed primary midbrain levels of the anti-apoptotic factor. It also might be the case that the nature of toxin used (MPTP vs. paraquat) is relevant in this regard.

In light of the fact that depression and other co-morbid symptoms typically occur together with the motor disturbances in PD (Farabaugh et al., 2009), it was particularly interesting that paraquat reduced hippocampal BDNF expression. Indeed, substantial evidence has indicated that reduced BDNF levels can contribute to depressive-like behavioral effects, as well as deficits in neuroplasticity and cognitive functioning (Anisman et al., 2008; Heldt et al., 2007; Schmidt and Duman, 2007). Similarly, other recent studies showed that BDNF administration in mice promoted a range of antidepressant and anti-anxiety-like cellular (e.g., hippocampal neurogenesis) and behavioral (e.g., forced swim, anhedonia) responses (Gourley et al., 2008; Schmidt and Duman, 2010). While the precise mechanisms and neural substrates underlying the co-morbid non-motor symptoms in PD have yet to be fully established, it seems likely that inflammatory and neurodegenerative processes in stressor-sensitive cognitive and emotional brain regions (e.g., hippocampus, prefrontal cortex, locus coeruleus) are important.

Indeed, histological pathology, including accumulation of $\alpha$-synuclein, occurred within the hippocampus of PD patients (Bertrand et al., 2003; Galvin et al., 1999). Moreover, paraquat altered hippocampal monoamine activity, and promoted depressiveand- anxiety-like responses in mice (Litteljohn et al., 2009). The present investigation 
provides evidence that paraquat, and possibly other environmental toxins, could potentially affect emotional and cognitive processing by causing trophic factor reductions in hippocampal pathways acting in parallel with the nigrostriatal system. Importantly, GM-CSF appeared to reverse the paraquat-induced hippocampal BDNF reduction, suggesting that its protective effects were not specific for nigrostriatal functioning but also translated across brain regions.

The present study provides early evidence in favor of future work focused on the potential use of GM-CSF as a therapeutic agent for PD. Although these findings do not fully elucidate a mechanism of action, it is clear that the neuroprotective effects of both systemic and central GM-CSF administration are accompanied by a blunting of the neuroinflammatory glial response provoked by LPS and paraquat. This is important in light of the accumulating evidence suggesting a primary role for microglial-driven inflammation in the death of DA neurons. However, disturbances of mitochondrial functioning, generation of oxidative radicals and promotion of apoptotic factors are probably the most proximal events in the neurodegenerative process. In this regard, it is apparent that GM-CSF is likely acting independently of the classical Bax and Bcl-2 apoptotic pathways. Yet, it is particularly notable that paraquat reduced BDNF within both the SNc and hippocampus, and that GM-CSF moderately reversed both of these effects. In summary, paraquat could conceivably contribute to motor and non-motor (e.g., depression, cognitive disturbance) PD symptoms by enhancing inflammatory processes and altering neuroplasticity, and GM-CSF might have important mitigating effects in this regard. Whatever the case, inflammatory priming (using LPS) of the SNc clearly increased DA neuronal vulnerability to the impact of later paraquat exposure and GM- 
CSF administration can prevent these effects when delivered either centrally or systemically. 
Proinflammatory cytokines and Parkinson's disease, 172

Chapter 5

General Discussion 


\section{General Discussion}

Over the past decade, numerous epidemiological and animal studies have implicated environmental insults, particularly pesticides, heavy metals and immune infections in the pathophysiology of Parkinson's disease (PD) (Dick et al., 2007; Weisskopf et al., 2010). Systemic exposures to the pesticide paraquat was reported to induce a dose-dependent loss of dopamine (DA) neurons in the substantia nigra pars compacta (SNc) of mice (McCormack et al., 2002), coupled with a reduction in the density of striatal DA fibres (Brooks et al., 1999). In agreement, the present thesis found that paraquat induced marked nigrostriatal degeneration in mice; however, these effects varied as a function of time and were greatly modified by manipulating the inflammatory immune system.

Paraquat has been shown to diminish striatal DA concentration and reproduce aspects of the PD phenotype (e.g. bradykinesia and deficits in motor coordination) in mice (Brooks et al., 1999; Chanyachukul et al., 2004; Chen et al., 2008). However, it is worth noting that the impact of paraquat upon the striatum appears to be somewhat less pronounced than its effects upon the SNc DA soma, thus some of the discrepancies between SNc pathology and striatal functioning have been described. Specifically, some studies failed to find a reduction in striatal DA levels or behavioural impairments despite a loss of DA soma (Thiruchelvam et al., 2003). It is conceivable that compensatory downstream processes provoked by soma loss (e.g. changes in dendritic branching patterns, up-regulation of neurotrophins, or alterations of brain monoamine systems) could account for such discrepancies. 
In addition to having greater epidemiological validity than the more classical DA toxin models of PD (6-OHDA and MPTP) paraquat and rotenone can induce histopathological changes (deposition of $\alpha$-synuclein aggregates) that more closely resemble the disease (Goers et al., 2003; Manning-Bog et al., 2002). Combinations of heavy metals and pesticides have been shown to synergistically provoke conformational changes in $\alpha$-synuclein, favouring the development of PD-like pathology in mice (Uversky et al., 2002). In fact, recent work revealed that exposure to a combination of iron and paraquat synergistically increased $\alpha$-synuclein aggregation and fibrillization, and augmented the extent of microglia-induced oxidative stress and neurodegeneration (Peng et al., 2010). Similarly, although the fungicide maneb had no effect on SNc DA neurons alone, when co-administered with paraquat it synergistically enhanced nigrostriatal damage and associated glial reactivity (Thiruchelvam et al., 2000b). Collectively these studies are in line with a recent hypothesis (referred to as the "multi-hit hypothesis") which suggests that idiopathic PD stems from multiple exposures to differing types of toxins superimposed upon some intrinsic genetic vulnerability.

The experiments described in the present thesis support the notion that microglia orchestrate a central inflammatory reaction within the nigrostriatal region in response to immunological and environmental insults, thus creating a pro-inflammatory and prooxidative state that can facilitate neurodegeneration. To this end, the ensuing sections of this discussion will describe the principle findings of these experiments.

Essentially, three separate manipulations were introduced that all support a role for microglia in modulating dopamine neuronal survival in the face a chemical or immune toxin: 1 . Genetic deletion of IFN- $\gamma$ (see Chapter 2) reduced the pro-inflammatory 
state (i.e. M1) of microglia and the corresponding loss of DA neurons; 2. The timing between inflammatory priming (LPS) and paraquat exposure determines the degree of microglial reactivity and nigrostriatal lesion (see Chapter 3); and 3. The trophic cytokine (GM-CSF) likewise promoted a more M2-like protective microglial state, coupled with reduced DA neuron loss (see Chapter 4). It should be noted that, besides its trophic actions, GM-CSF has well documented pro-inflammatory effects. However, proinflammatory effects within the CNS following GM-CSF treatment are usually not observed until doses much higher than ours are used (Hamilton, 2002)

\section{Paraquat and clinical aspects of Parkinson's disease}

Traditionally, clinical signs of the disease are thought to manifest only after approximately $80 \%$ of DA neurons have been lost (Bernheimer et al 1973); however, more recent findings have challenged this position. In fact, a number of reports suggest that mild and more subtle forms of the cognitive and motor symptoms may be evident in PD patients long before clinical diagnosis (Brooks et al., 1999). We recently found that paraquat in mice exposed to the toxin for 1-week ( 3 injections) was not sufficient to induce motor deficits in the pole test and open field, which are indicative of PD-like syndrome, whereas 3 -weeks (9 injections) was sufficient to provoke such outcomes (Litteljohn et al., 2009). These behavioural results corresponded to the amount of time required for paraquat to cause $\mathrm{SNc}$ degeneration (see Chapter 2).

Several additional signs of motor deficits illustrated using a rating scale were evident following 3-weeks of paraquat (see Chapter 3). It may be argued that a subset of these symptoms (e.g. impaired gait, reduced locomotion, and poor posture) stem from a systemic toxicity following paraquat, given that paraquat does affect peripheral organ functioning, most notable the lungs (Shopova et al., 2009; Suntres, 2002). However, if 
the current paraquat paradigm induced such systemic toxicity, it would be expected that mice would display signs of general illness and respiratory dysfunction. To assess this possibility, all mice were rated for signs of sickness (e.g. ptosis, piloerection) and breathing patterns were evaluated prior to motor testing/rating using a standard rating scale validated in mice (Cicchetti et al., 2005; Mangano and Hayley, 2009). To this end, no obvious signs of sickness were evident in the present investigation nor were there any indications of pulmonary disturbances upon visual inspection of mice. Thus, the present results strongly support the hypothesis that paraquat produces a Parkinsonian-like syndrome in mice.

\section{Parkinson's disease and inflammation}

Until fairly recently the brain was believed to function more or less independently of the immune system. However, it is now accepted that circulating T-lymphocytes, macrophages and other peripheral immune cells routinely enter the brain, albeit in limited concentrations and perform a variety of "housekeeping" tasks essential for immunosurveillance (Wilson et al., 2010). In the case of inflammatory diseases such as multiple sclerosis (MS), which is characterized by profound disturbances in brain-immune communication, it is hypothesized that there is a breakdown in immunosurveillance. Consequently, through a faulty blood brain barrier (BBB) CNS defences are weakened resulting in the infiltration of excessive numbers of cytotoxic T-lymphocytes into the brain parenchyma. In contrast to MS, PD is likely not a primary inflammatory disease in the same classical sense. In fact, changes in BBB functioning or trafficking of peripheral immune cells appear to be more subtle in PD. However, several recent reports have detected some degree of immune infiltration (albeit less than that of MS) and it has even 
been posited that $\mathrm{M} 1$ reactive microglia might recruit circulating (in the blood and CSF) $\mathrm{CD} 4^{+}$T-lymphocytes to the SNc to modulate dopaminergic survival (Appel et al., 2010; Brochard et al., 2009; Reynolds et al., 2009). Whatever the case, it has become unequivocally clear that marked changes in immunocompetent microglial cells occur in the midbrain region of PD patients and in animals treated with DA targeting toxins.

\section{Microglia are primary mediators of nigrostriatal damage}

Two days after a single supra-SNc infusion of the bacterial endotoxin, LPS, we show that microglial cells undergo profound morphological changes and express a proinflammatory phenotype, indicated by compacted and amoeboid-like shape (Chapter 3 ). This morphological change indicates that these cells have reached their most extreme activated state, wherein they produce potentially deleterious oxidative and inflammatory factors. This change from a resting / anti-inflammatory to pro-inflammatory has recently been characterized as a phenotypic shift from a baseline neuroprotective role (M2) to a neurotoxic role (M1) for microglia. Indeed, anti-inflammatory (M2) microglial cells are typically associated with angiogenesis and the release of anti-inflammatory cytokines and trophic factors, (Michelucci et al., 2009). In contrast, pro-inflammatory (M1) microglial cells are normally characterized by the production of oxidative metabolites (e.g., nitric oxide, superoxide) and pro-inflammatory cytokines that are essential for innate immunity and have been associated with surrounding damage to healthy cells/tissue (Pawate et al., 2004; Qin et al., 2004).

Further experiments revealed that when mice commenced paraquat treatments at a time of heightened microglial (M1) activity, the magnitude of the nigrostriatal lesion was significantly magnified (Chapter 3 ). This finding clearly supports the contention that a 
pro-inflammatory state can sensitize DA neurons to the impact of later environmental toxin exposures. Interestingly, however, when paraquat exposure commenced 7-days after LPS priming (when microglia reactivity began to return to control levels) there actually was some degree of neuroprotection (Chapter 3). Specifically, nigrostriatal DA neurons appeared to be protected from the impact of paraquat both at the level of the SNc and striatum. We hypothesized that this 7-day delay allows time for compensatory processes to be mounted. For instance, microglia may now become refractory while astrocytes and even neurons may begin to up-regulate heat shock proteins and growth factors, such as GDNF and BDNF. In short, it appears that the timing between a proinflammatory insult, which involves the recruitment of microglia and subsequent exposure to environmental toxicants, is critical in determining the nature of the neurodegenerative properties.

The concept of time-dependent sensitization and desensitization effects influencing neuronal survival is widely studied in stroke research. Indeed, it has become well established that a brief mild cerebral ischemic event can actually protect neurons from subsequent larger ischemic challenges, a phenomenon referred to as ischemic tolerance (Gidday, 2006). Interestingly, ischemic tolerance is highly time-dependent, such that although protection is observed when relatively short intervals of 1-2 days are interspersed between the ischemic events, longer intervals (e.g. 7-14 days) actually resulted in an augmented cerebral damage (Gidday, 2006). Thus, much like the timing between the employed LPS and paraquat regimen, certain time intervals resulted in a sensitized response (increased damage), while other intervals favoured a desensitization (protective effect). 
It is useful to note that ischemic tolerance has been associated with the release of anti-inflammatory factors, whereas pro-inflammatory factors are associated with exacerbation of cerebral damage (i.e. after longer re-exposure interval). For instance, the pro-inflammatory cytokine $\mathrm{TNF}-\alpha$ dose-dependently exacerbated the degree of cortical neuronal loss caused by middle cerebral artery occlusion and this damage was prevented when TNF- $\alpha$ receptors were blocked prior to the stroke (Barone et al., 1997). Timedependent changes in BBB permeability can differentially affect the degree of damage caused by an ischemic attack with prolonged and/or delayed BBB permeability promoting an influx of numerous peripheral leukocytes over time, which can facilitate damage to neuronal populations (Fisher 2008).

Importantly, M1 microglial cells have been hypothesized to recruit peripheral antigen presenting cells (e.g. macrophages and dendritic cells) into the CNS following injury (Glass et al., 2010) and recruitment of these peripheral immune cells would likely enhance the degree of neurodegeneration by their ability to produce oxidative and other deleterious factors (Glass et al., 2010). On the other hand, it is also possible that the recruitment of peripheral antigen presenting cells would serve protective functions at certain times by scavenging debris, removing pathogens, releasing trophic factors and buffering ionic disturbances in the extracellular space. Hence, it is reasonable to suggest that microglia might exert a myriad of time-dependent effects, some of which might be beneficial and others more detrimental.

As in the case of cerebral ischemia, microglia may have distinct time-dependent functions relative to chronic paraquat exposures. As reported in Chapter 2, microglia only begins to express oxidative factors (NADPH oxidase and iNOS) 3 weeks into the 
paraquat regimen. In contrast, the trophic factor BDNF and its upstream transcription factor CREB were reduced earlier, at 1-week during the paraquat regimen. Although we did not directly assess BDNF and CREB within microglia, it is known that these factors are produced by both microglia and astrocytes (Murer et al., 2001). It is also interesting to note that pro-inflammatory factors cytokines (e.g. TNF- $\alpha$, IL-6 and IL-1 $\beta$ ) and enzymes (e.g. COX-2) were enhanced after 3 weeks, but not after 1 week of paraquat exposure. It is well known that microglia are the main CNS reservoir of these inflammatory factors and as such, we speculate that only at later stages of the paraquat regimen do microglia really adopt the most deleterious pro-inflammatory M1-like phenotype. It is important to note that the microglia changes induced in Chapter 2 were evident with paraquat exposure in the absence of LPS inflammatory priming. Thus, the pesticide itself had marked inflammatory and oxidative effects and the LPS priming used in subsequent chapters served to further reinforce such effects.

While the intermediate signalling stages of microglia are still not fully resolved, it is clear that oxidative radicals are a critical proximal mediator of cell death induced by paraquat. Since, paraquat is a redox cycler capable of generating large amounts of superoxide by electron transfer reactions with microglial-NADPH oxidase (Day et al., 1999; Pawate et al., 2004; Ramachandiran et al., 2007) and the degenerative effects of most DA neurotoxins can be completely prevented using mice lacking a functional NADPH oxidase or by administering pharmacological inhibitors for iNOS and NADPH prior to the administration of a toxin (Delgado, 2003; Gao et al., 2003b; Gupta et al., 2010; Iravani et al., 2005; Le et al., 2001; Liberatore et al., 1999), these pro-oxidant factors are likely to be involved in the degenerative processes caused by paraquat. Taken 
together, these data support a primary rather a secondary role for microglia in responding to environmental insults.

Cytokines and nigrostriatal degeneration: $I L-1 \beta$ and $T N F-\alpha$

The current thesis revealed that LPS induced a transient increase (within 90 minutes) of the pro-inflammatory cytokines IL-1 $\beta$ and TNF- $\alpha$ within the plasma and SNc (Chapter 3). Not unexpectedly, this spike in protein concentration was short-lived, returning to basal levels by 2-days. We postulate that this early rise may contribute to the initiation of a phenotypic shift of SNc microglial cells from their basal anti-inflammatory (M2) phenotype to a more pro-inflammatory (M1) (Chapters 3 and 4). Hence, these cytokines may facilitate neurodegeneration indirectly through their ability to modulate microglia's phenotypic state.

It has been proposed that $\mathrm{TNF}-\alpha$ is a "master inflammatory regulator", influencing the production of pro-inflammatory cytokines, microgliosis, BBB dysfunction, cell adhesion and neuroinflammation (Kraft et al., 2009). Given this and the fact that microglia are the primary source of TNF- $\alpha$, we postulate that TNF- $\alpha$ is necessary for maintaining a chronic pro-inflammatory state and is necessary for promoting ongoing degeneration. Along these lines, repeated exposure to paraquat for 3-weeks but not 1week was associated with delayed activation of the pro-inflammatory cytokines TNF- $\alpha$ and IL-1 $\beta$ as well as the pro-oxidants iNOS and NADPH oxidase (Chapter 2). It is relevant to recall that that 1 -week of paraquat was not sufficient to elevate these proinflammatory mediators or cause degeneration; only after 3-weeks of paraquat were the DA neurons lost (Chapter 2). 
It remains possible that the delayed increase in the aforementioned proinflammatory cytokines is the result of repeated paraquat exposures. Specifically, chronic pesticide exposures may eventually overwhelm endogenous anti-oxidants causing an accumulation of reactive oxygen species (ROS) within the SNc causing damage to DA neurons. Subsequently, damaged DA neurons can recruit further local microglia to the site of injury to assist in either the restoration or removal of these damaged neurons. Upon recruiting local microglia, TNF- $\alpha$ and IL-1 $\beta$ may be released in an attempt to remove the sick/dying DA neurons. In effect, although we expect that an initial primary microglia response might be engendered by the toxins themselves, secondary responses by these cells to deal with the ongoing pathology are unavoidable. Thus, the nature of the glial response (e.g. neuroprotective versus neurodestructive) may vary over time following the insult. It might be actually be viewed as being to overly simplistic in stated a simple binary "good" versus "bad" role for microglia. Whatever the case, complex temporal cytokine variations would ultimately be expected to shape the functioning of microglia and alter the inflammatory milieu.

It has been postulated that the amount of TNF- $\alpha$ released from microglia and the neuronal expression pattern of TNF-Rs can be a determining factor for neuronal death and survival (Kraft et al., 2009). Essentially, in addition to acting directly upon microglia and promoting an M1 phenotype, TNF- $\alpha$ may directly bind to DA neurons and promote apoptotic cell death. Indeed, immunohistochemical analysis of human brains revealed that TNF-R1 but not TNF-R2, are expressed on SNc dopaminergic neurons (McCoy and Tansey, 2008). Even though the precise function of TNF- $\alpha$ is not completely understood, several in vivo and in vitro studies using TNF-R1 and TNF-R2 KO mice indicate that 
TNF-R1 can be toxic to neurons while TNF-R2 is neuroprotective (Fontaine et al., 2002; Kassiotis and Kollias, 2001).

Despite the fact that the data presented in the thesis regarding the function of TNF- $\alpha$ are correlative, several studies have demonstrated that activation of this cytokine occurs prior to ischemia-induced cellular death in vitro models using LPS (Botchkina et al., 1999; Mander and Brown, 2005; Shibata et al., 2003). Nonetheless, to fully confirm our hypothesis that TNF- $\alpha$ is instrumental to the neurodegenerative response induced by LPS and paraquat further studies will be necessary to characterize the activational timecourse of TNF- $\alpha$. Future studies illustrating that TNF- $\alpha$ is activated prior to DA cell death together with studies showing that blocking TNF- $\alpha$ can prevent ongoing cell death would provide compelling evidence for the involvement of TNF- $\alpha$ in the neurodegenerative response.

Given that the downstream signalling pathway for IL-1 $\beta$ converges with the proapoptotic TNF-R1 pathway using the pro-apoptotic transcription factors NF-kB and cJun, we postulate that IL-1 $\beta$ is working in conjunction with TNF- $\alpha$ to induce the death of DA neurons caused by paraquat. Indeed, neutralizing antibodies to either TNF- $\alpha$ or IL-1 $\beta$ can protect dopaminergic neurons from the neurotoxic effects of LPS in vitro, thus supporting the hypothesis that TNF- $\alpha$ and IL-1 $\beta$ are working together to promote cell death (Gayle et al., 2002). Delayed elevations in these pro-inflammatory cytokines following 3-weeks of paraquat were accompanied by an increase in the transcription factors $\mathrm{NK}$ and NFkB (Chapter 2). This is observation is particularly intriguing given that one of the critical signalling factors required for TNF- $\alpha$ induced apoptosis is JNK (Hayley et al., 2004a; Sakon et al., 2003). 
Cytokines and nigrostriatal degeneration: IFN- $\gamma$

Although IL-1 $\beta$ and TNF- $\alpha$ are the cytokines probably most commonly implicated in neuropathological processes, IFN- $\gamma$ is actually the most potent in provoking a pro-inflammatory microglial response. IFN- $\gamma$ predominately up-regulates anti-microbial mechanisms as well as antigen presentation pathways and can facilitate the recruitment of leukocytes, direct growth, maturation and differentiation of many cell types involved in innate and adaptive immune responses (Schroder et al., 2004). Many of the known microglial-dependent pro-inflammatory and pro-oxidative genes are subjected to regulatory control by IFN- $\gamma$ (Glass et al., 2010). For instance, IFN- $\gamma$-associated microglial JAK/STAT signalling mediates the up-regulated or de novo synthesis of several proteins critical for microglial antigen presentation and generation of the superoxide respiratory burst (Kawanokuchi et al., 2006).

It will be recalled that IFN- $\gamma$ acts primarily through the JAK/STAT signalling pathways causing homodimeric STAT complexes (primarily STAT1) to translocate to the nucleus and induce the transcription of myriad IFN- $\gamma$-responsive genes (Briscoe et al., 1996). Correspondingly, we found that paraquat increased the SNc expression of STAT1 in a time-dependent fashion (Chapter 2). The majority of available evidence suggests that IFN- $\gamma$ likely influences neuronal survival and promotes oxidative stress through its actions on microglia. Indeed, we found that IFN- $\gamma$ deficient mice displayed reduced microglial activation, which corresponded to neuroprotection from the impact of paraquat. However, one recent study did report that IFN- $\gamma$ can trigger glutamate excitotoxic neuronal damage (e.g., dendritic beading) by activating a unique complex consisting of an IFN- $\gamma$ receptor and an AMPA receptor GluR1, which together form a 
$\mathrm{Ca}^{2+}$-permeable receptor complex, which subsequently, allows enhanced $\mathrm{Ca}^{2+}$ entry into cortical neurons leading to nitric oxide production and eventual reduction of intracellular ATP levels by inhibition of mitochondrial respiration (Mizuno et al., 2008).

The genes encoding for the pro-oxidants iNOS and NADPH oxidase, as well as the dsRNA protein kinase (PKR) gene, which is necessary component in antiviral defence are all governed by IFN- $\gamma$ induced JAK/STAT signalling (Pawate et al., 2004; Pyo et al., 2008; Zamanian-Daryoush et al., 2000). Specifically, IFN- $\gamma$ induced translocation of STAT1 homodimers to up-regulate the genes encoding for NADPH oxidase and iNOS, which will promote oxidative stress by enhancing the production of superoxide and nitric oxide, respectively. Similarly, up-regulation of the PKR gene will induce microglial-COX-2 activation through IFN- $\gamma$ 's actions on the NF- $\mathrm{KB}$ signalling pathway (Zamanian-Daryoush et al., 2000). Our findings in Chapter 2 are in agreement given that genetic deletion of the cytokine attenuated the effects of paraquat upon iNOS, $\mathrm{NADPH}$ subunits and COX-2. Moreover, pre-treatment with the hormone melatonin, attenuated the induction of iNOS and COX-2 by LPS/IFN- $\gamma$, and this effect was attributed to the inhibition of NF-אB activation (Deng et al., 2006). Accordingly, IFN- $\gamma$ may impact neuronal survival by way of its downstream effects on key microglial enzymes implicated in the elaboration of deleterious inflammatory factors (i.e. nitric oxide, ROS and prostanoids).

It has been postulated that IFN- $\gamma$ plays an important role in the initial stages of infection recognition (during the innate response). It is likely that early activation of IFN$\gamma$ by antigen presenting cells, such as macrophages, dendritic cells and microglia, acts as an early initiator of a pro-inflammatory cascade, allowing these immune cells to respond 
to invading pathogens (Schroder et al., 2004). The pro-inflammatory cytokines, IL-12 and IL-18 are up-stream of IFN- $\gamma$-associated JAK/STAT signalling, where they facilitate the transition of an innate to adaptive immune response through IFN- $\gamma$ (Frucht et al., 2001). Indeed, each of these cytokines (including IFN- $\gamma$ itself) can promote an adaptive immune response skewing $\mathrm{CD}^{+}$T-lymphocyte development towards a Th1/proinflammatory phenotype, which is, in fact, consistent with post-mortem PD brains (Baba et al., 2005; Brochard et al., 2009).

Lastly, IFN- $\gamma$-associated JAK/STAT signalling has also been shown to downregulate several ostensibly neuroprotective species in microglial cells. For instance, IFN$\gamma$ dampened microglial expression of the secretory protein osteopontin, which has antiapoptotic properties (Moran et al., 2007). Interestingly, osteopontin has been shown to attenuate the neurodegenerative consequences of stroke and various neurotoxins (Doyle et al., 2008; Iczkiewicz et al., 2006; Iczkiewicz et al., 2005; Meller et al., 2005). Whatever the case, the present thesis furthers the notion that IFN- $\gamma$ might be a critical player in controlling the microglial phenotype in response to DA toxins, and hence, has important implications for PD.

\section{Anti-inflammatory and trophic cytokines protect nigrostriatal neurons}

Healthy individuals typically exhibit a more anti-inflammatory microglial phenotype (M2) that plays a role in maintaining a homeostatic equilibrium within the brain. In contrast to M1 state microglia, M2 cells typically secrete anti-inflammatory cytokines such as IL-4 and IL-10, which together with TGF-B and glucocorticoids have been recently demonstrated to be negative regulators of IFN- $\gamma$ (Schroder et al., 2004). 
In addition to the involvement of M1 microglia and pro-inflammatory cytokines, paraquat also induced a reduction in astrocytic support (Chapters 2-4). A reduction in astrocytic support may increase the vulnerability of DA neurons to repeated neurotoxic insults by reducing the availability of trophic support (Howells et al., 2000; Murer et al., 2001). Prior to the death of DA neurons, paraquat caused an early reduction in both CREB and BDNF (Chapter 2). This early reduction may provide an explanation as to why DA neurons become vulnerable to the continued administration of paraquat. However, the molecular mechanism by which this trophic factor influences (if at all) PD progression is poorly understood (Zuccato and Cattaneo, 2009), and whether a reduction of BDNF and its downstream consequences is a causative factor in PD remains inconclusive. It is possible that this reduction is simply the outcome caused by a reduction in the number of DA neurons. This is a reasonable hypothesis given that $\mathrm{SNc}$ DA neurons have been shown to contain BDNF (Baquet et al., 2005).

The current thesis demonstrated that paraquat-induced reduction in SNc CREB and BDNF occurred prior to the degeneration of DA neurons, see Chapter 2. Moreover, the neuroprotective properties of GM-CSF following paraquat were associated with a partial restoration SNc BDNF (Chapter 4). We postulate that paraquat caused a reduction of astrocytic support, which results in a decrease in the amount of BDNF released. Hence, it would be useful to conduct future studies to determine if restoring the astrocytic population would in effect restore BDNF levels and protect the SNc.

One of the major obstacles with translating neurotrophic factor research from the bench to the bedside involves the difficulties in the delivery approach since penetrating the $\mathrm{BBB}$ is extremely challenging. Increasing endogenous BDNF or introducing 
exogenous BDNF may be a valid treatment option for PD. However, clinical trials using BDNF in Amyotrophic Lateral Sclerosis (ALS) demonstrated that many problems, including low BBB permeability, short in vivo half-life and limited diffusion (Zuccato and Cattaneo, 2009). Before a viable BDNF therapy can be developed for PD, new protocols accurately quantifying the amount of BDNF proteins levels in human brains need to be established. One major obstacle is that the procedures presently used to assess BDNF in post-mortem brains may not reflect BDNF in vivo processing. Because BDNF is sensitive to a wide range of stimuli including dietary restrictions, physical exercise, circadian rhythms and stress (Zuccato and Cattaneo, 2009), a full understanding of BDNF physiology is critical for understanding bioavailability requirements of a BDNF treatment. It is also important to note that administration of an "incorrect" dose of BDNF can be detrimental, given that excessive amounts of BDNF can have deleterious effects on neural circuitry, learning and memory (Croll et al., 1999).

The trophic cytokine, GM-CSF, likely promotes microglial to adopt a more neuroprotective and anti-inflammatory phenotype, likely inducing the release of BDNF (Bouhy et al., 2006; Hayashi et al., 2009; and see Chapter 4). However, several recent studies have also demonstrated an anti-apoptotic role for GM-CSF in several CNS pathologies. This is not surprising given that GM-CSF has been shown in several CNS disorders (i.e. animal model of PD, stroke and spinal cord injury) to enhance gene expression of the anti-apoptotic protein Bcl-2 (Choi et al.; Kim et al., 2009b; Kong et al., 2009). In a more recent report, GM-CSF has also been shown to inhibit the expression of pro-apoptotic proteins p53, Bax and caspases-3 following cerebral ischemia (Kong et al., 2009). These reports are in agreement with the current thesis, as GM-CSF not only 
attenuated the effects of LPS and paraquat, but these results were correlated with an enhanced gene expression of Bcl-2 (Chapter 4). For the most part, long-term neuroprotective effects of GM-CSF and the mechanism through which GM-CSF is capable of regulating these pro-apoptotic proteins have yet to be evaluated.

GM-CSF has been shown to cause a phenotypic shift in the activational state of microglia, from a more pro-inflammatory $\mathrm{Ml}$ to resemble dendritic-like peripheral immune cells (Liva et al., 1999). Indeed, we found that GM-CSF did appear to alter the morphological state of microglia following LPS and paraquat; however, whether they were more dendritic-like requires further investigation. Nonetheless, this hypothesis is interesting given that microglia can easily alter their activation state.

The signal transduction pathways meditating GM-CSF's neuroprotective effects are not fully understood. However, the JAK/ STAT pathway seems to be crucial for GMCSF induced hematopoietic proliferation. Specifically, it is clear that GM-CSF will activate the intracellular adaptor protein, JAK2, and signal through the transcription factor, STAT5 (Liva et al., 1999). In addition to promoting proliferation, JAK2 and STAT5A/B have been shown to induce Bcl-2 and delay cell death in a hematopoietic cell line (Sakai and Kraft, 1997). Therefore, it is possible that GM-CSF may prevent paraquat induced apoptosis through a JAK2 dependent pathway.

On the other hand, the ERK1/2 MAP kinase pathway may also be essential in mediating a proliferative response to GM-CSF. Recent reports have suggested that GMCSF will also activate the MAP kinase ERK1/2, in addition to a few other signalling molecules (Liva et al., 1999; Montenegro et al., 2009; Sakamoto et al., 1994). This is in 
agreement with our preliminary data, which demonstrates that GM-CSF can increase ERK1/2 alone and in response to LPS and paraquat, see Appendix A, Figure 1. This is interesting given that several reports have suggested that GM-CSF can reduce neutrophil and eosinophil induced apoptotic cell death through ERK1/2, as well as phosphatidylinositol 3-kinase (PI3)-3 kinase/ATK (Klein et al., 2000; Kotone-Miyahara et al., 2004). In contrast, Choi et al. (2010) demonstrated that exposure of GM-CSF to neural progenitor cells will up-regulate anti-apoptotic proteins (e.g. Bcl-2 and $\mathrm{Bcl-xL}$ ) using the JAK2/STAT5 pathway and not PI3-kinase/AKT or ERK1/2. These conflicting results may be a result of the cell types used (neural progenitor cells versus neutrophils and eosinophils).

Given that either administration of either GM-CSF or knocking out IFN- $\gamma$ resulted in neuroprotection (but likely through very different mechanisms) demonstrates the complexity of PD and suggests the potential for many avenues of intervention. Yet, both of these neuroprotective strategies had similar outcomes with regards to the inflammatory glial milieu. This is in accord with our contention that the neurotoxins used in the present thesis (LPS and paraquat) activate common inflammatory signalling pathways within the CNS, and these likely contribute to DA neuronal pathology. This is also in line with epidemiological reports that indicate chronic exposure to multiple environmental toxins over a prolonged period of time increase risk to developing PD (Abbott et al., 2003; Gorell et al., 2004; Priyadarshi et al., 2001).

\section{Conclusions}

The present thesis highlighted the importance of cytokine signalling not only as regulators of immune processes, but also as messengers involved in toxin induced 
nigrostriatal degeneration. Given that PD likely originates from the interactive effects of multiple environmental insults upon a possibly compromised genetic background, it is necessary to evaluate the joint effects of more than one causative agent. In this regard, the present data support the notion that a pre-existing neuroinflammatory state (induced by a virus or a bacterial infection) may promote some of the deleterious consequences of later toxin exposure(s). Finally, the assessment of multiple cytokines simultaneously, as conducted in the present thesis, may provide important clues as to how these pleiotropic immunotransmitters may interact over time to ultimately shape the evolution of disease states such as PD. 
Proinflammatory cytokines and Parkinson's disease, 192

Appendix A 


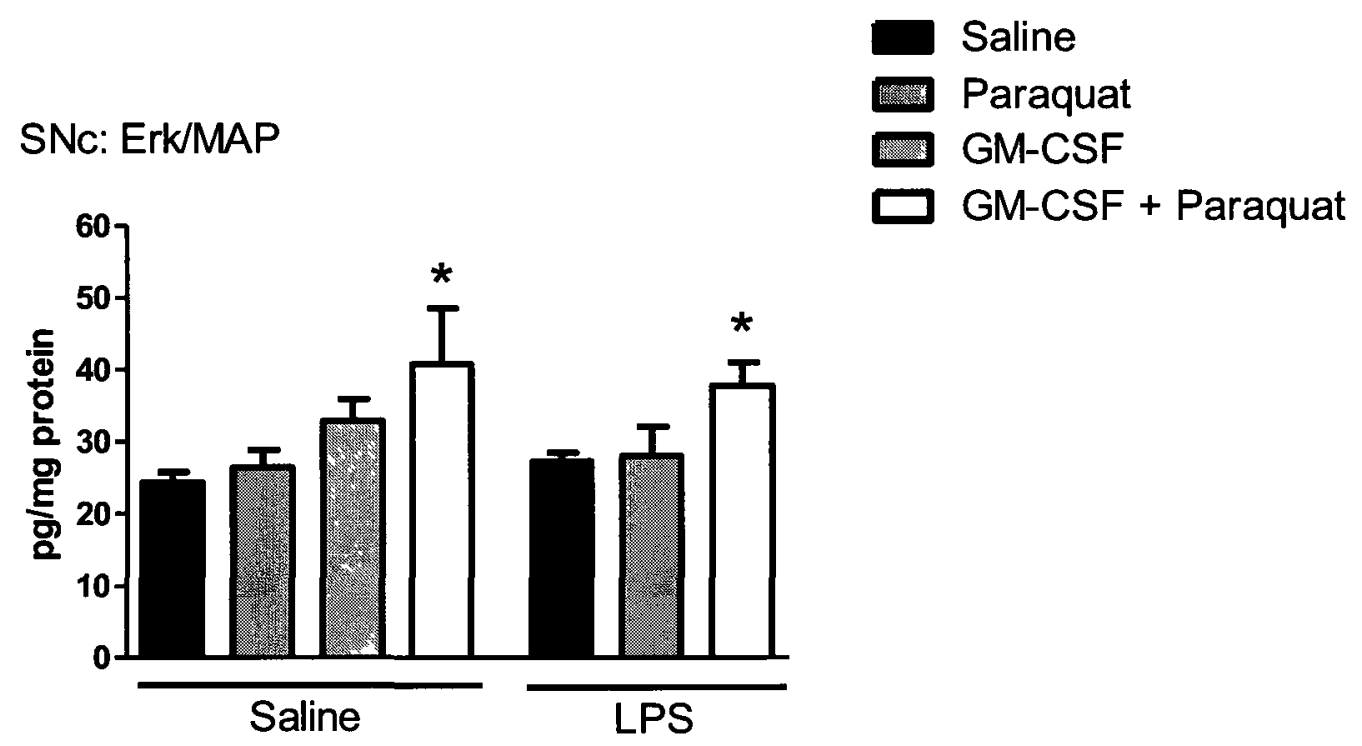

Figure 6.1 Central administration of GM-CSF elevated Erk1/2 within the SNc following LPS and paraquat.

The following mice were infused with a single supra-nigral injection of LPS $(0.01 \mu \mathrm{g} / 2$ l) 2 days prior to beginning the paraquat regimen $(10 \mathrm{mg} / \mathrm{kg}$; 3 per week for 3-weeks). A single supra-nigral injection of GM-CSF $(10 \mathrm{ng} / 2 \mu \mathrm{l})$ once per week during the paraquat regimen elevated phosphorylated Erk $1 / 2$ protein levels. Data are expressed as mean \pm SEM; $\mathrm{n}=8-10$. 


\section{References}

2007. Mixed lineage kinase inhibitor CEP-1347 fails to delay disability in early Parkinson disease. Neurology. 69, 1480-90.

Abbott, R. D., Ross, G. W., White, L. R., Sanderson, W. T., Burchfiel, C. M., Kashon, M., Sharp, D. S., Masaki, K. H., Curb, J. D., Petrovitch, H., 2003. Environmental, life-style, and physical precursors of clinical Parkinson's disease: recent findings from the Honolulu-Asia Aging Study. J Neurol. 250 Suppl 3, III30-9.

Ahmadi, F. A., Grammatopoulos, T. N., Poczobutt, A. M., Jones, S. M., Snell, L. D., Das, M., Zawada, W. M., 2008. Dopamine selectively sensitizes dopaminergic neurons to rotenone-induced apoptosis. Neurochem Res. 33, 886-901.

Ai, Y., Markesbery, W., Zhang, Z., Grondin, R., Elseberry, D., Gerhardt, G. A., Gash, D. M., 2003. Intraputamenal infusion of GDNF in aged rhesus monkeys: distribution and dopaminergic effects. J Comp Neurol. 461, 250-61.

Alam, Z. I., Jenner, A., Daniel, S. E., Lees, A. J., Cairns, N., Marsden, C. D., Jenner, P., Halliwell, B., 1997. Oxidative DNA damage in the parkinsonian brain: an apparent selective increase in 8-hydroxyguanine levels in substantia nigra. J Neurochem. 69, 1196-203.

Aloisi, F., 2001. Immune function of microglia. Glia. 36, 165-79.

Andersen, J. K., 2003a. Paraquat and iron exposure as possible synergistic environmental risk factors in Parkinson's disease. Neurotox Res. 5, 307-13.

Andersen, J. K., 2003b. Paraquat and iron exposure as possible synergistic environmental risk factors in parkinson's disease. Neurotox Res. 5, 307-313. 
Anisman, H., Merali, Z., Hayley, S., 2008. Neurotransmitter, peptide and cytokine processes in relation to depressive disorder: comorbidity between depression and neurodegenerative disorders. Prog Neurobiol. 85, 1-74.

Anrather, J., Racchumi, G., Iadecola, C., 2006. NF-kappaB regulates phagocytic NADPH oxidase by inducing the expression of gp91phox. J Biol Chem. 281, 5657-67.

Aoki, E., Yano, R., Yokoyama, H., Kato, H., Araki, T., 2009. Role of nuclear transcription factor kappa B (NF-kappaB) for MPTP (1-methyl-4-phenyl-1,2,3,6tetrahyropyridine)-induced apoptosis in nigral neurons of mice. Exp Mol Pathol. $86,57-64$.

Appel, S. H., Beers, D. R., Henkel, J. S., 2010. T cell-microglial dialogue in Parkinson's disease and amyotrophic lateral sclerosis: are we listening? Trends Immunol. 31, 7-17.

Arimoto, T., Choi, D. Y., Lu, X., Liu, M., Nguyen, X. V., Zheng, N., Stewart, C. A., Kim, H. C., Bing, G., 2006. Interleukin-10 protects against inflammationmediated degeneration of dopaminergic neurons in substantia nigra. Neurobiol Aging.

Audoy-Remus, J., Richard, J. F., Soulet, D., Zhou, H., Kubes, P., Vallieres, L., 2008. Rod-Shaped monocytes patrol the brain vasculature and give rise to perivascular macrophages under the influence of proinflammatory cytokines and angiopoietin2. J Neurosci. 28, 10187-99.

Baba, Y., Kuroiwa, A., Uitti, R. J., Wszolek, Z. K., Yamada, T., 2005. Alterations of Tlymphocyte populations in Parkinson disease. Parkinsonism \& related disorders. $11,493-498$. 
Baldi, I., Cantagrel, A., Lebailly, P., Tison, F., Dubroca, B., Chrysostome, V., Dartigues, J. F., Brochard, P., 2003. Association between Parkinson's disease and exposure to pesticides in southwestern France. Neuroepidemiology. 22, 305-10.

Baquet, Z. C., Bickford, P. C., Jones, K. R., 2005. Brain-derived neurotrophic factor is required for the establishment of the proper number of dopaminergic neurons in the substantia nigra pars compacta. J Neurosci. 25, 6251-9.

Barcia, C., Sanchez Bahillo, A., Fernandez-Villalba, E., Bautista, V., Poza, Y. P. M., Fernandez-Barreiro, A., Hirsch, E. C., Herrero, M. T., 2004. Evidence of active microglia in substantia nigra pars compacta of parkinsonian monkeys 1 year after MPTP exposure. Glia. 46, 402-9.

Barone, F. C., 2005. Endogenous brain protection: models, gene expression, and mechanisms. Methods Mol Med. 104, 105-84.

Barone, F. C., Arvin, B., White, R. F., Miller, A., Webb, C. L., Willette, R. N., Lysko, P. G., Feuerstein, G. Z., 1997. Tumor necrosis factor-alpha. A mediator of focal ischemic brain injury. Stroke. $28,1233-44$.

Barone, F. C., White, R. F., Spera, P. A., Ellison, J., Currie, R. W., Wang, X., Feuerstein, G. Z., 1998. Ischemic preconditioning and brain tolerance: temporal histological and functional outcomes, protein synthesis requirement, and interleukin-1 receptor antagonist and early gene expression. Stroke; a journal of cerebral circulation. 29, 1937-50; discussion 1950-1.

Bartels, A. L., Kortekaas, R., Bart, J., Willemsen, A. T., de Klerk, O. L., de Vries, J. J., van Oostrom, J. C., Leenders, K. L., 2009. Blood-brain barrier P-glycoprotein 
function decreases in specific brain regions with aging: a possible role in progressive neurodegeneration. Neurobiol Aging. 30, 1818-24.

Bartels, A. L., van Berckel, B. N., Lubberink, M., Luurtsema, G., Lammertsma, A. A., Leenders, K. L., 2008a. Blood-brain barrier P-glycoprotein function is not impaired in early Parkinson's disease. Parkinsonism Relat Disord. 14, 505-8.

Bartels, A. L., Willemsen, A. T., Kortekaas, R., de Jong, B. M., de Vries, R., de Klerk, O., van Oostrom, J. C., Portman, A., Leenders, K. L., 2008b. Decreased bloodbrain barrier P-glycoprotein function in the progression of Parkinson's disease, PSP and MSA. J Neural Transm. 115, 1001-9.

Basic Kes, V., Simundic, A. M., Nikolac, N., Topic, E., Demarin, V., 2008. Proinflammatory and anti-inflammatory cytokines in acute ischemic stroke and their relation to early neurological deficit and stroke outcome. Clin Biochem. 41, 13304.

Behrstock, S., Ebert, A., McHugh, J., Vosberg, S., Moore, J., Schneider, B., Capowski, E., Hei, D., Kordower, J., Aebischer, P., Svendsen, C. N., 2006. Human neural progenitors deliver glial cell line-derived neurotrophic factor to parkinsonian rodents and aged primates. Gene Ther. 13, 379-88.

Bell, R. D., Zlokovic, B. V., 2009. Neurovascular mechanisms and blood-brain barrier disorder in Alzheimer's disease. Acta Neuropathol. 118, 103-13.

Bennani-Baiti, N., Davis, M. P., 2008. Cytokines and cancer anorexia cachexia syndrome. Am J Hosp Palliat Care. 25, 407-11.

Benner, E. J., Mosley, R. L., Destache, C. J., Lewis, T. B., Jackson-Lewis, V., Gorantla, S., Nemachek, C., Green, S. R., Przedborski, S., Gendelman, H. E., 2004. 
Therapeutic immunization protects dopaminergic neurons in a mouse model of Parkinson's disease. Proc Natl Acad Sci USA. 101, 9435-9440.

Bergman, H., Raz, A., Feingold, A., Nini, A., Nelken, I., Hansel, D., Ben-Pazi, H., Reches, A., 1998. Physiology of MPTP tremor. Mov Disord. 13 Supp1 3, 29-34.

Bernacki, J., Dobrowolska, A., Nierwinska, K., Malecki, A., 2008. Physiology and pharmacological role of the blood-brain barrier. Pharmacol Rep. 60, 600-22.

Bertrand, E., Lechowicz, W., Lewandowska, E., Szpak, G. M., Dymecki, J., KosnoKruszewska, E., Wierzba-Bobrowicz, T., 2003. Degenerative axonal changes in the hippocampus and amygdala in Parkinson's disease. Folia Neuropathol. 41, 197-207.

Bessis, A., Bechade, C., Bernard, D., Roumier, A., 2007. Microglial control of neuronal death and synaptic properties. Glia. 55, 233-8.

Betarbet, R., Canet-Aviles, R. M., Sherer, T. B., Mastroberardino, P. G., McLendon, C., Kim, J. H., Lund, S., Na, H. M., Taylor, G., Bence, N. F., Kopito, R., Seo, B. B., Yagi, T., Yagi, A., Klinefelter, G., Cookson, M. R., Greenamyre, J. T., $2006 a$. Intersecting pathways to neurodegeneration in Parkinson's disease: effects of the pesticide rotenone on DJ-1, alpha-synuclein, and the ubiquitin-proteasome system. Neurobiol Dis. 22, 404-420.

Betarbet, R., Canet-Aviles, R. M., Sherer, T. B., Mastroberardino, P. G., McLendon, C., Kim, J. H., Lund, S., Na, H. M., Taylor, G., Bence, N. F., Kopito, R., Seo, B. B., Yagi, T., Yagi, A., Klinefelter, G., Cookson, M. R., Greenamyre, J. T., $2006 \mathrm{~b}$. Intersecting pathways to neurodegeneration in Parkinson's disease: effects of the 
pesticide rotenone on $\mathrm{DJ}-1$, alpha-synuclein, and the ubiquitin-proteasome system. Neurobiol Dis. 22, 404-20.

Betarbet, R., Sherer, T. B., Di Monte, D. A., Greenamyre, J. T., 2002. Mechanistic approaches to Parkinson's disease pathogensis. Brain Pathol. 12, 499-510.

Betarbet, R., Sherer, T. B., MacKenzie, G., Garcia-Osuna, M., Panov, A. V., Greenamyre, J. T., 2000. Chronic systemic pesticide exposure reproduces features of Parkinson's disease. Nature neuroscience. 3, 1301-1306.

Bezard, E., Jaber, M., Gonon, F., Boireau, A., Bloch, B., Gross, C. E., 2000. Adaptive changes in the nigrostriatal pathway in response to increased 1-methyl-4-phenyl1,2,3,6-tetrahydropyridine-induced neurodegeneration in the mouse. Eur $\mathbf{J}$ Neurosci. 12, 2892-900.

Bharath, S., Hsu, M., Kaur, D., Rajagopalan, S., Andersen, J. K., 2002. Glutathione, iron and Parkinson's disease. Biochem Pharmacol. 64, 1037-48.

Bian, M. J., Li, L. M., Yu, M., Fei, J., Huang, F., 2009. Elevated interleukin-1beta induced by 1-methyl-4-phenyl-1,2,3,6-tetrahydropyridine aggravating dopaminergic neurodegeneration in old male mice. Brain Res. 1302, 256-64.

Blandini, F., Nappi, G., Tassorelli, C., Martignoni, E., 2000. Functional changes of the basal ganglia circuitry in Parkinson's disease. Progress in neurobiology. 62, 6388.

Block, M. L., Hong, J. S., 2005. Microglia and inflammation-mediated neurodegeneration: multiple triggers with a common mechanism. Prog Neurobiol. $76,77-98$. 
Block, M. L., Zecca, L., Hong, J. S., 2007. Microglia-mediated neurotoxicity: uncovering the molecular mechanisms. Nat Rev Neurosci. 8, 57-69.

Blum, D., Torch, S., Lambeng, N., Nissou, M., Benabid, A. L., Sadoul, R., Verna, J. M., 2001. Molecular pathways involved in the neurotoxicity of 6-OHDA, dopamine and MPTP: contribution to the apoptotic theory in Parkinson's disease. Progress in neurobiology. 65, 135-172.

Bolin, L. M., Strycharska-Orczyk, I., Murray, R., Langston, J. W., Di Monte, D., 2002. Increased vulnerability of dopaminergic neurons in MPTP-lesioned interleukin-6 deficient mice. Journal of neurochemistry. 83, 167-175.

Bolin, L. M., Zhaung, A., Strychkarska-Orczyk, I., Nelson, E., Huang, I., Malit, M., Nguyen, Q., 2005. Differential inflammatory activation of IL-6 (-/) astrocytes. Cytokine. 30, 47-55.

Bolli, R., Dawn, B., Xuan, Y. T., 2003. Role of the JAK-STAT pathway in protection against myocardial ischemia/reperfusion injury. Trends Cardiovasc Med. 13, 729.

Bossers, K., Meerhoff, G., Balesar, R., van Dongen, J. W., Kruse, C. G., Swaab, D. F., Verhaagen, J., 2009. Analysis of gene expression in Parkinson's disease: possible involvement of neurotrophic support and axon guidance in dopaminergic cell death. Brain Pathol. 19, 91-107.

Botchkina, G. I., Geimonen, E., Bilof, M. L., Villarreal, O., Tracey, K. J., 1999. Loss of NF-kappaB activity during cerebral ischemia and TNF cytotoxicity. Mol Med. 5, $372-81$. 
Bouhy, D., Malgrange, B., Multon, S., Poirrier, A. L., Scholtes, F., Schoenen, J., Franzen, R., 2006. Delayed GM-CSF treatment stimulates axonal regeneration and functional recovery in paraplegic rats via an increased BDNF expression by endogenous macrophages. FASEB J. 20, 1239-41.

Boulet, S., Mounayar, S., Poupard, A., Bertrand, A., Jan, C., Pessiglione, M., Hirsch, E. C., Feuerstein, C., Francois, C., Feger, J., Savasta, M., Tremblay, L., 2008. Behavioral recovery in MPTP-treated monkeys: neurochemical mechanisms studied by intrastriatal microdialysis. J Neurosci. 28, 9575-84.

Bove, J., Prou, D., Perier, C., Przedborski, S., 2005. Toxin-induced models of Parkinson's disease. NeuroRx. 2, 484-94.

Briscoe, J., Guschin, D., Rogers, N. C., Watling, D., Muller, M., Horn, F., Heinrich, P., Stark, G. R., Kerr, I. M., 1996. JAKs, STATs and signal transduction in response to the interferons and other cytokines. Philos Trans R Soc Lond B Biol Sci. 351, $167-71$.

Brochard, V., Combadiere, B., Prigent, A., Laouar, Y., Perrin, A., Beray-Berthat, V., Bonduelle, O., Alvarez-Fischer, D., Callebert, J., Launay, J. M., Duyckaerts, C., Flavell, R. A., Hirsch, E. C., Hunot, S., 2009. Infiltration of CD4+ lymphocytes into the brain contributes to neurodegeneration in a mouse model of Parkinson disease. J Clin Invest. 119, 182-92.

Brodacki, B., Staszewski, J., Toczylowska, B., Kozlowska, E., Drela, N., Chalimoniuk, M., Stepien, A., 2008. Serum interleukin (IL-2, IL-10, IL-6, IL-4), TNFalpha, and INFgamma concentrations are elevated in patients with atypical and idiopathic parkinsonism. Neurosci Lett. 441, 158-62. 
Bronstein, J. M., Tagliati, M., Alterman, R. L., Lozano, A. M., Volkmann, J., Stefani, A., Horak, F. B., Okun, M. S., Foote, K. D., Krack, P., Pahwa, R., Henderson, J. M., Hariz, M. I., Bakay, R. A., Rezai, A., Marks, W. J., Jr., Moro, E., Vitek, J. L., Weaver, F. M., Gross, R. E., Delong, M. R., Deep Brain Stimulation for Parkinson Disease: An Expert Consensus and Review of Key Issues. Arch Neurol.

Brooks, A. I., Chadwick, C. A., Gelbard, H. A., Cory-Slechta, D. A., Federoff, H. J., 1999a. Paraquat elicited neurobehavioral syndrome caused by dopaminergic neuron loss. Brain Res. 823, 1-10.

Brown, D. S., Belfield, A. J., Brown, G. R., Campbell, D., Foubister, A., Masters, D. J., Pike, K. G., Snelson, W. L., Wells, S. L., 2004. A novel series of p38 MAP kinase inhibitors for the potential treatment of rheumatoid arthritis. Bioorg Med Chem Lett. 14, 5383-7.

Brown, T. P., Rumsby, P. C., Capleton, A. C., Rushton, L., Levy, L. S., 2006. Pesticides and Parkinson's disease--is there a link? Environ Health Perspect. 114, 156-64.

Bruce, A. J., Boling, W., Kindy, M. S., Peschon, J., Kraemer, P. J., Carpenter, M. K., Holtsberg, F. W., Mattson, M. P., 1996. Altered neuronal and microglial responses to excitotoxic and ischemic brain injury in mice lacking TNF receptors. Nat Med. 2, 788-94.

Bruck, A., Kurki, T., Kaasinen, V., Vahlberg, T., Rinne, J. O., 2004. Hippocampal and prefrontal atrophy in patients with early non-demented Parkinson's disease is related to cognitive impairment. J Neurol Neurosurg Psychiatry. 75, 1467-9. 
Butcher, E. C., 1991. Leukocyte-endothelial cell recognition: three (or more) steps to specificity and diversity. Cell. 67, 1033-6.

Cannon, J. R., Tapias, V., Na, H. M., Honick, A. S., Drolet, R. E., Greenamyre, J. T., 2009. A highly reproducible rotenone model of Parkinson's disease. Neurobiol Dis. $34,279-90$.

Cardenas, H., Bolin, L. M., 2003. Compromised reactive microgliosis in MPTP-lesioned IL-6 KO mice. Brain Res. 985, 89-97.

Carrasco, E., Casper, D., Werner, P., 2005. Dopaminergic neurotoxicity by 6-OHDA and MPP+: differential requirement for neuronal cyclooxygenase activity. Journal of neuroscience research. 81, 121-131.

Carvey, P. M., Chang, Q., Lipton, J. W., Ling, Z., 2003. Prenatal exposure to the bacteriotoxin lipopolysaccharide leads to long-term losses of dopamine neurons in offspring: a potential, new model of Parkinson's disease. Frontiers in bioscience [computer file] : a journal and virtual library. 8, s826-37.

Carvey, P. M., Punati, A., Newman, M. B., 2006. Progressive dopamine neuron loss in Parkinon's disease: the multiple hit hypothesis. Cell Transplant. 15, 239-250.

Casals, J., Elizan, T. S., Yahr, M. D., 1998. Postencephalitic parkinsonism--a review. Journal of neural transmission (Vienna, Austria : 1996). 105, 645-676.

Cassarejos, M. J., Menendez, J., Solano, R. M., Rodriguez-Navarro, J. A., Garcia de Yebenes, J., Mena, M. A., 2006. Susceptibility to rotenone is increased in neurons from parkin null mice and is reduced by minocycline. J Neurochem. 97, 934-946.

Castano, A., Herrera, A. J., Cano, J., Machado, A., 2002. The degenerative effect of a single intranigral injection of LPS on the dopaminergic system is prevented by 
dexamethasone, and not mimicked by rh-TNF-alpha, IL-1beta and IFN-gamma. Journal of neurochemistry. $81,150-157$.

Caudle, W. M., Richardson, J. R., Wang, M. Z., Taylor, T. N., Guillot, T. S., McCormack, A. L., Colebrooke, R. E., Di Monte, D. A., Emson, P. C., Miller, G. W., 2007. Reduced vesicular storage of dopamine causes progressive nigrostriatal neurodegeneration. J Neurosci. 27, 8138-48.

Chanyachukul, T., Yoovathaworn, K., Thongsaard, W., Chongthammakun, S., Navasumrit, P., Satayavivad, J., 2004. Attenuation of paraquat-induced motor behavior and neurochemical disturbances by L-valine in vivo. Toxicol Lett. 150, $259-69$.

Chauhan, N. B., Siegel, G. J., Lee, J. M., 2001. Depletion of glial cell line-derived neurotrophic factor in substantia nigra neurons of Parkinson's disease brain. $\mathbf{J}$ Chem Neuroanat. 21, 277-88.

Chen, C. M., Liu, J. L., Wu, Y. R., Chen, Y. C., Cheng, H. S., Cheng, M. L., Chiu, D. T., 2009. Increased oxidative damage in peripheral blood correlates with severity of Parkinson's disease. Neurobiol Dis. 33, 429-35.

Chen, P., Chen, Z., Li, A., Lou, X. C., Wu, X. K., Zhao, C. J., Wang, S. L., Liang, L. P., 2008. Catalytic metalloporphyrin protects against paraquat neurotoxicity in vivo. Biomed Environ Sci. 21, 233-8.

Chen, P. S., Peng, G. S., Li, G., Yang, S., Wu, X., Wang, C. C., Wilson, B., Lu, R. B., Gean, P. W., Chuang, D. M., Hong, J. S., 2006. Valproate protects dopaminergic neurons in midbrain neuron/glia cultures by stimulating the release of neurotrophic factors from astrocytes. Mol Psychiatry. 11, 1116-25. 
Chen, Q., Niu, Y., Zhang, R., Guo, H., Gao, Y., Li, Y., Liu, R., 2010. The toxic influence of paraquat on hippocampus of mice: involvement of oxidative stress. Neurotoxicology. 31, 310-6.

Cheret, C., Gervais, A., Lelli, A., Colin, C., Amar, L., Ravassard, P., Mallet, J., Cumano, A., Krause, K. H., Mallat, M., 2008. Neurotoxic activation of microglia is promoted by a nox 1-dependent NADPH oxidase. J Neurosci. 28, 12039-51.

Chesler, D. A., McCutcheon, J. A., Reiss, C. S., 2004. Posttranscriptional regulation of neuronal nitric oxide synthase expression by IFN-gamma. J Interferon Cytokine Res. 24, 141-9.

Choi, J. K., Kim, K. H., Park, H., Park, S. R., Choi, B. H., Granulocyte macrophagecolony stimulating factor shows anti-apoptotic activity in neural progenitor cells via JAK/STAT5-Bcl-2 pathway. Apoptosis.

Choi, S., Yeum, C. H., Kim, Y. D., Park, C. G., Kim, M. Y., Park, J. S., Jeong, H. S., Kim, B. J., So, I., Kim, K. W., 2010. Receptor tyrosine and MAP kinase are involved in effects of $\mathrm{H}(2) \mathrm{O}(2)$ on interstitial cells of Cajal in murine intestine. J Cell Mol Med. 14, 257-66.

Choi, W. S., Abel, G., Klintworth, H., Flavell, R. A., Xia, Z., JNK3 mediates paraquatand rotenone-induced dopaminergic neuron death. J Neuropathol Exp Neurol. 69, $511-20$.

Cicchetti, F., Drouin-Ouellet, J., Gross, R. E., 2009. Environmental toxins and Parkinson's disease: what have we learned from pesticide-induced animal models? Trends Pharmacol Sci. 30, 475-83. 
Cicchetti, F., Lapointe, N., Roberge-Tremblay, A., Saint-Pierre, M., Jimenez, L., Ficke, B. W., Gross, R. E., 2005. Systemic exposure to paraquat and maneb models early Parkinson's disease in young adult rats. Neurobiology of Disease. 20, 360-371.

Ciesielska, A., Joniec, I., Przybylkowski, A., Gromadzka, G., Kurkowska-Jastrzebska, I., Czlonkowska, A., Czlonkowski, A., 2003. Dynamics of expression of the mRNA for cytokines and inducible nitric synthase in a murine model of the Parkinson's disease. Acta Neurobiol Exp. 63, 117-126.

Collier, T. J., Lipton, J., Daley, B. F., Palfi, S., Chu, Y., Sortwell, C., Bakay, R. A., Sladek, J. R., Jr., Kordower, J. H., 2007. Aging-related changes in the nigrostriatal dopamine system and the response to MPTP in nonhuman primates: diminished compensatory mechanisms as a prelude to parkinsonism. Neurobiol Dis. 26, 56-65.

Conde, J. R., Streit, W. J., 2006a. Effect of aging on the microglial response to peripheral nerve injury. Neurobiol Aging. 27, 1451-61.

Conde, J. R., Streit, W. J., 2006b. Microglia in the aging brain. J Neuropathol Exp Neurol. 65, 199-203.

Cory-Slechta, D. A., Thiruchelvam, M., Barlow, B. K., Richfield, E. K., 2005. Developmental pesticide models of the Parkinson disease phenotype. Environ Health Perspect. 113, 1263-70.

Costello, S., Cockburn, M., Bronstein, J., Zhang, X., Ritz, B., 2009. Parkinson's disease and residential exposure to maneb and paraquat from agricultural applications in the central valley of California. Am J Epidemiol. 169, 919-26. 
Cotrina, M. L., Lin, J. H., Lopez-Garcia, J. C., Naus, C. C., Nedergaard, M., 2000. ATPmediated glia signaling. J Neurosci. 20, 2835-44.

Croll, S. D., Suri, C., Compton, D. L., Simmons, M. V., Yancopoulos, G. D., Lindsay, R. M., Wiegand, S. J., Rudge, J. S., Scharfman, H. E., 1999. Brain-derived neurotrophic factor transgenic mice exhibit passive avoidance deficits, increased seizure severity and in vitro hyperexcitability in the hippocampus and entorhinal cortex. Neuroscience. 93, 1491-506.

Cunningham, C., Wilcockson, D. C., Campion, S., Lunnon, K., Perry, V. H., 2005. Central and systemic endotoxin challenges exacerbate the local inflammatory response and increase neuronal death during chronic neurodegenation. J Neurosci. $25,9245-9284$.

Czlonkowska, A., Kurkowska-Jastrzebska, I., Czlonkowski, A., Peter, D., Stefano, G. B., 2002. Immune processes in the pathogenesis of Parkinson's disease - a potential role for microglia and nitric oxide. Medical science monitor : international medical journal of experimental and clinical research. 8, RA165-77.

Czlonkowska, M., Knhutnicka, M., Kurowska-Jastrzebska, I., Czlonkowski, A., 1996. Microglial reaction in MPTP (1-methyl-4-1,2,3,6-tetrahydropyridine) induced Parkinson's disease mice model. Neurodegeneration. 5, 137-143.

Dale, D. C., 2002. Colony-stimulating factors for the management of neutropenia in cancer patients. Drugs. 62 Suppl 1, 1-15.

Dalton, D. K., Pitts-Meek, S., Keshav, S., Figari, I. S., Bradley, A., Stewart, T. A., 1993. Multiple defects of immune cell function in mice with disrupted interferongamma genes. Science. 259, 1739-42. 
Dang, P. M., Morel, F., Gougerot-Pocidalo, M. A., El Benna, J., 2003. Phosphorylation of the NADPH oxidase component p67(PHOX) by ERK2 and P38MAPK: selectivity of phosphorylated sites and existence of an intramolecular regulatory domain in the tetratricopeptide-rich region. Biochemistry. 42, 4520-6.

Davalos, D., Grutzendler, J., Yang, G., Kim, J. V., Zuo, Y., Jung, S., Littman, D. R., Dustin, M. L., Gan, W. B., 2005. ATP mediates rapid microglial response to local brain injury in vivo. Nat Neurosci. $8,752-8$.

Davignon, J. L., Kimoto, M., Kindler, V., De Kossodo, S., Vassalli, P., Izui, S., 1988. Selective production of interleukin 3 (IL3) and granulocyte-macrophage colonystimulating factor (GM-CSF) in vitro by murine L3T4+ T cells: lack of spontaneous IL3 and GM-CSF production by Ly-2-/L3T4- lpr subset. Eur J Immunol. 18, 1367-72.

Davis, R. J., 2000. Signal transduction by the JNK group of MAP kinases. Cell. 103, 23952.

Dawson, T. M., Dawson, V. L., 2003. Rare genetic mutations shed light on the pathogenesis of Parkinson's disease. J Clin Invest. 111, 145-51.

Day, B. J., Patel, M., Calavetta, L., Chang, L. Y., Stamler, J. S., 1999. A mechanism of paraquat toxicity involving nitric oxide synthase. Proceedings of the National Academy of Sciences of the United States of America. 96, 12760-12765.

de Boer, A. G., Gaillard, P. J., 2006. Blood-brain barrier dysfunction and recovery. Journal of neural transmission (Vienna, Austria : 1996). 113, 455-462.

De Lella Ezcurra, A. L., Chertoff, M., Ferrari, C., Graciarena, M., Pitossi, F., Chronic expression of low levels of tumor necrosis factor-alpha in the substantia nigra 
elicits progressive neurodegeneration, delayed motor symptoms and microglia/macrophage activation. Neurobiol Dis. 37, 630-40.

de Prati, A. C., Ciampa, A. R., Cavalieri, E., Zaffini, R., Darra, E., Menegazzi, M., Suzuki, H., Mariotto, S., 2005. STAT1 as a new molecular target of antiinflammatory treatment. Curr Med Chem. 12, 1819-28.

Delgado, M., 2003. Inhibition of interferon (IFN) gamma-induced Jak-STAT1 activation in microglia by vasoactive intestinal peptide: inhibitory effect on CD40, IFNinduced protein-10, and inducible nitric-oxide synthase expression. J Biol Chem. $278,27620-9$.

DeLong, M. R., Wichmann, T., 2007. Circuits and circuit disorders of the basal ganglia. Arch Neurol. 64, $20-4$.

Deng, W. G., Tang, S. T., Tseng, H. P., Wu, K. K., 2006. Melatonin suppresses macrophage cyclooxygenase- 2 and inducible nitric oxide synthase expression by inhibiting p52 acetylation and binding. Blood. 108, 518-24.

Depino, A. M., Earl, C., Kaczmarczyk, E., Ferrari, C., Besedovsky, H., del Rey, A., Pitossi, F. J., Oertel, W. H., 2003. Microglial activation with atypical proinflammatory cytokine expression in a rat model of Parkinson's disease. The European journal of neuroscience. 18, 2731-2742.

Dexter, D. T., Carter, C. J., Wells, F. R., Javoy-Agid, F., Agid, Y., Lees, A., Jenner, P., Marsden, C. D., 1989. Basal lipid peroxidation in substantia nigra is increased in Parkinson's disease. J Neurochem. 52, 381-9. 
Dexter, D. T., Holley, A. E., Flitter, W. D., Slater, T. F., Wells, F. R., Daniel, S. E., Lees, A. J., Jenner, P., Marsden, C. D., 1994. Increased levels of lipid hydroperoxides in the parkinsonian substantia nigra: an HPLC and ESR study. Mov Disord. 9, 92-7.

Dhillon, A. S., Tarbutton, G. L., Levin, J. L., Plotkin, G. M., Lowry, L. K., Nalbone, J. T., Shepherd, S., 2008. Pesticide/environmental exposures and Parkinson's disease in East Texas. J Agromedicine. 13, 37-48.

Di Monte, D. A., 2003. The environment and Parkinson's disease: is the nigrostriatal system preferentially targeted by neurotoxins? Lancet Neurol. 2, 531-8.

Diaz-Hernadez, M., Hernandez, F., Martin-Aparicio, E., Gomez-Ramos, P., Moran, M. A., Castano, J. G., Ferrer, I., Avila, J., Lucas, J. J., 2003. Neuronal induction of the immunoproteasome in Huntington's disease. J Neurosci. 23, 11653-11661.

Dick, F. D., De Palma, G., Ahmadi, A., Scott, N. W., Prescott, G. J., Bennett, J., Semple, S., Dick, S., Counsell, C., Mozzoni, P., Haites, N., Wettinger, S. B., Mutti, A., Otelea, M., Seaton, A., Soderkvist, P., Felice, A., 2007. Environmental risk factors for Parkinson's disease and parkinsonism: the Geoparkinson study. Occup Environ Med. 64, 666-72.

Dickman, M. S., 2001. Von economo enchephalitis. Arch Neurol. 2001, 10.

Dickstein, J. B., Moldofsky, H., Hay, J. B., 2000. Brain-blood permeability: TNF-alpha promotes escape of protein tracer from CSF to blood. Am J Physiol Regul Integr Comp Physiol. 279, R148-51.

Dinarello, C. A., 1988. Biology of interleukin 1. FASEB J. 2, 108-15.

Dobbie, M. S., Hurst, R. D., Klein, N. J., Surtees, R. A., 1999. Upregulation of intercellular adhesion molecule-1 expression on human endothelial cells by 
tumour necrosis factor-alpha in an in vitro model of the blood-brain barrier. Brain Res. 830, 330-6.

Doherty, F. J., Dawson, S., Mayer, R. J., 2002. The ubiquitin-proteasome pathway of intracellular proteolysis. Essays Biochem. 38, 51-63.

Doolittle, N. D., Miner, M. E., Hall, W. A., Siegal, T., Jerome, E., Osztie, E., McAllister, L. D., Bubalo, J. S., Kraemer, D. F., Fortin, D., Nixon, R., Muldoon, L. L., Neuwelt, E. A., 2000. Safety and efficacy of a multicenter study using intraarterial chemotherapy in conjunction with osmotic opening of the blood-brain barrier for the treatment of patients with malignant brain tumors. Cancer. 88, 63747.

Dore-Duffy, P., 2008. Pericytes: pluripotent cells of the blood brain barrier. Curr Pharm Des. 14, 1581-93.

Doyle, K. P., Yang, T., Lessov, N. S., Ciesielski, T. M., Stevens, S. L., Simon, R. P., King, J. S., Stenzel-Poore, M. P., 2008. Nasal administration of osteopontin peptide mimetics confers neuroprotection in stroke. J Cereb Blood Flow Metab. $28,1235-48$

Dringen, R., 2005. Oxidative and antioxidative potential of brain microglial cells. Antioxid Redox Signal. 7, 1223-33.

Du, Y., Ma, Z., Lin, S., Dodel, R. C., Gao, F., Bales, K. R., Triarhou, L. C., Chernet, E., Perry, K. W., Nelson, D. L., Luecke, S., Phebus, L. A., Bymaster, F. P., Paul, S. M., 2001. Minocycline prevents nigrostriatal dopaminergic neurodegeneration in the MPTP model of Parkinson's disease. Proceedings of the National Academy of Sciences of the United States of America. 98, 14669-14674. 
Dunnett, S. B., Bjorklund, A., 1999. Prospects for new restorative and neuroprotective treatments in Parkinson's disease. Nature. 399, A32-9.

Dutheil, F., Beaune, P., Tzourio, C., Loriot, M. A., Elbaz, A., Interaction between $\mathrm{ABCB} 1$ and professional exposure to organochlorine insecticides in Parkinson disease. Arch Neurol. 67, 739-45.

Eberling, J. L., Bankiewicz, K. S., Jordan, S., VanBrocklin, H. F., Jagust, W. J., 1997. PET studies of functional compensation in a primate model of Parkinson's disease. Neuroreport. 8, 2727-33.

Eigler, A., Sinha, B., Hartmann, G., Endres, S., 1997. Taming TNF: strategies to restrain this proinflammatory cytokine. Immunol Today. 18, 487-92.

Eklund, E. A., Kakar, R., 1999. Recruitment of CREB-binding protein by PU.1, IFNregulatory factor-1, and the IFN consensus sequence-binding protein is necessary for IFN-gamma-induced p67phox and gp9lphox expression. J Immunol. 163, 6095-105.

Elbaz, A., Clavel, J., Rathouz, P. J., Moisan, F., Galanaud, J. P., Delemotte, B., Alperovitch, A., Tzourio, C., 2009. Professional exposure to pesticides and Parkinson disease. Ann Neurol. 66, 494-504.

Eslamboli, A., 2005. Assessment of GDNF in primate models of Parkinson's disease: comparison with human studies. Rev Neurosci. 16, 303-10.

Farabaugh, A. H., Locascio, J. J., Yap, L., Weintraub, D., McDonald, W. M., Agoston, M., Alpert, J. E., Growdon, J., Fava, M., 2009. Pattern of depressive symptoms in Parkinson's disease. Psychosomatics. 50, 448-54. 
Farkas, I. G., Czigner, A., Farkas, E., Dobo, E., Soos, K., Penke, B., Endresz, V., Mihaly, A., 2003. Beta-amyloid peptide-induced blood-brain barrier disruption facilitates T-cell entry into the rat brain. Acta Histochem. 105, 115-125.

Farrall, A. J., Wardlaw, J. M., 2009. Blood-brain barrier: ageing and microvascular disease--systematic review and meta-analysis. Neurobiol Aging. 30, 337-52.

Faulkner, J. R., Herrmann, J. E., Woo, M. J., Tansey, K. E., Doan, N. B., Sofroniew, M. V., 2004. Reactive astrocytes protect tissue and preserve function after spinal cord injury. J Neurosci. 24, 2143-55.

Fawcett, J. W., Asher, R. A., 1999. The glial scar and central nervous system repair. Brain Res Bull. 49, 377-91.

Fehling, H. J., Swat, W., Laplace, C., Kuhn, R., Rajewsky, K., Muller, U., von Boehmer, H., 1994. MHC class I expression in mice lacking the proteasome subunit LMP-7. Science. 265, 1234-1237.

Fei, Q., Ethell, D. W., 2008. Maneb potentiates paraquat neurotoxicity by inducing key Bcl-2 family members. J Neurochem.

Feng, Z., Li, D., Fung, P. C., Pei, Z., Ramsden, D. B., Ho, S. L., 2003. COX-2-deficient mice are less prone to MPTP-neurotoxicity than wild-type mice. Neuroreport. 14, $1927-9$.

Ferger, B., Leng, A., Mura, A., Hengerer, B., Feldon, J., 2004. Genetic ablation of turmor necrosis factor-alpha (TNF-a) and pharmacological inhibition of TNF-a synthesis attenuates MPTP toxicity in mouse striatum. J Neurochem. 89, 822-833.

Fernagut, P. O., Hutson, C. B., Fleming, S. M., Tetreaut, N. A., Salcedo, J., Masliah, E., Chesselet, M. F., 2007. Behavioral and histopathological consequences of 
paraquat intoxication in mice: effects of alpha-synuclein over-expression. Synapse. 61, 991-1001.

Fischer, H. G., Reichmann, G., 2001. Brain dendritic cells and macrophages/microglia in central nervous system inflammation. J Immunol. 166, 2717-26.

Fischer, M, 2008. Injuries to the vascular endothelium: vascular wall and endothelial dysfunction. Rev Neurol Disease. 5 Suppl 1:S4-11.

Fitzmaurice, P. S., Ang, L., Guttman, M., Rajput, A. H., Furukawa, Y., Kish, S. J., 2003. Nigral glutathione deficiency is not specific for idiopathic Parkinson's disease. Mov Disord. 18, 969-76.

Fontaine, V., Mohand-Said, S., Hanoteau, N., Fuchs, C., Pfizenmaier, K., Eisel, U., 2002. Neurodegenerative and neuroprotective effects of tumor Necrosis factor (TNF) in retinal ischemia: opposite roles of TNF receptor 1 and TNF receptor 2 . $J$ Neurosci. 22, RC216.

Forno, L. S., DeLanney, L. E., Irwin, I., Langston, J. W., 1994. Evolution of nerve fiber degeneration in the striatum in the MPTP-treated squirrel monkey. Mol Neurobiol. 9, 163-70.

Fox, C. M., Gash, D. M., Smoot, M. K., Cass, W. A., 2001. Neuroprotective effects of GDNF against 6-OHDA in young and aged rats. Brain Res. 896, 56-63.

Franzen, R., Bouhy, D., Schoenen, J., 2004. Nervous system injury: focus on the inflammatory cytokine 'granulocyte-macrophage colony stimulating factor'. Neurosci Lett. 361, 76-8. 
Frucht, D. M., Fukao, T., Bogdan, C., Schindler, H., O'Shea, J. J., Koyasu, S., 2001. IFNgamma production by antigen-presenting cells: mechanisms emerge. Trends Immunol. 22, 556-60.

Funayama, M., Hattori, N., 2007. [Molecular genetics of PINK1]. Brain Nerve. 59, 8318.

Galvin, J. E., Uryu, K., Lee, V. M., Trojanowski, J. Q., 1999. Axon pathology in Parkinson's disease and Lewy body dementia hippocampus contains alpha-, beta-, and gamma-synuclein. Proc Natl Acad Sci U S A. 96, 13450-5.

Gao, H. M., Hong, J. S., 2008. Why neurodegenerative diseases are progressive: uncontrolled inflammation drives disease progression. Trends Immunol. 29, 35765.

Gao, H. M., Hong, J. S., Zhang, W., Liu, B., 2002a. Distinct role for microglia in rotenone-induced degeneration of dopaminergic neurons. The Journal of neuroscience : the official journal of the Society for Neuroscience. 22, 782-790.

Gao, H. M., Hong, J. S., Zhang, W., Liu, B., 2003a. Synergistic dopaminergic neurotoxicity of the pesticide rotenone and inflammation lipopolysaccharide: relevance to the etiology of Parkinson's disease. J Neurosci. 23, 1228-1236.

Gao, H. M., Jiang, J., Wilson, B., Zhang, W., Hong, J. S., Liu, B., 2002b. Microglial activation-mediated delayed and progressive degeneration of rat nigral dopaminergic neurons: relevance to Parkinson's disease. Journal of neurochemistry. $81,1285-1297$. 
Gao, H. M., Liu, B., Hong, J. S., 2003b. Critical role for microglial NADPH oxidase in rotenone-induced degeneration of dopaminergic neurons. The Journal of neuroscience : the official journal of the Society for Neuroscience. 23, 6181-6187.

Gao, H. M., Liu, B., Zhang, W., Hong, J. S., 2003c. Synergistic dopaminergic neurotoxicity of MPTP and inflammogen lipopolysaccharide: relevance to the etiology of Parkinson's disease. The FASEB journal : official publication of the Federation of American Societies for Experimental Biology. 17, 1957-1959.

Gash, D. M., Zhang, Z., Gerhardt, G., 1998. Neuroprotective and neurorestorative properties of GDNF. Ann Neurol. 44, S121-5.

Gash, D. M., Zhang, Z., Ovadia, A., Cass, W. A., Yi, A., Simmerman, L., Russell, D., Martin, D., Lapchak, P. A., Collins, F., Hoffer, B. J., Gerhardt, G. A., 1996. Functional recovery in parkinsonian monkeys treated with GDNF. Nature. 380, $252-5$.

Gatto, N. M., Cockburn, M., Bronstein, J., Manthripragada, A. D., Ritz, B., 2009. Wellwater consumption and Parkinson's disease in rural California. Environ Health Perspect. 117, 1912-8.

Gayle, D. A., Ling, Z., Tong, C., Landers, T., Lipton, J. W., Carvey, P. M., 2002. Lipopolysaccharide (LPS)-induced dopamine cell loss in culture: roles of tumor necrosis factor-alpha, interleukin-lbeta, and nitric oxide. Brain research. Developmental brain research. 133, 27-35.

German, D. C., Nelson, E. L., Liang, C. L., Speciale, S. G., Sinton, C. M., Sonsalla, P. K., 1996. The neurotoxin MPTP causes degeneration of specific nucleus A8, A9 and A10 dopaminergic neurons in the mouse. Neurodegeneration. 5, 299-312. 
Gidday, J. M., 2006. Cerebral preconditioning and ischaemic tolerance. Nature Reviews Neuroscience. 7, 437-448.

Gill, S. S., Patel, N. K., Hotton, G. R., O'Sullivan, K., McCarter, R., Bunnage, M., Brooks, D. J., Svendsen, C. N., Heywood, P., 2003. Direct brain infusion of glial cell line-derived neurotrophic factor in Parkinson disease. Nat Med. 9, 589-95.

Glass, C. K., Saijo, K., Winner, B., Marchetto, M. C., Gage, F. H., 2010. Mechanisms Underlying Inflammation in Neurodegeneration. Cell. 140, 918-934.

Glezer, I., Simard, A. R., Rivest, S., 2007. Neuroprotective role of the innate immune system by microglia. Neuroscience. 147, 867-83.

Goers, J., Manning-Bog, A. B., McCormack, A. L., Millett, I. S., Doniach, S., Di Monte, D. A., Uversky, V. N., Fink, A. L., 2003. Nuclear localization of alpha-synuclein and its interaction with histones. Biochemistry. 42, 8465-71.

Gomez-Lazaro, M., Galindo, M. F., Concannon, C. G., Segura, M. F., Fernandez-Gomez, F. J., Llecha, N., Comella, J. X., Prehn, J. H., Jordan, J., 2008. 6Hydroxydopamine activates the mitochondrial apoptosis pathway through p38 MAPK-mediated, p53-independent activation of Bax and PUMA. J Neurochem. $104,1599-612$.

Gomez-Pinilla, F., Lee, J. W., Cotman, C. W., 1992. Basic FGF in adult rat brain: cellular distribution and response to entorhinal lesion and fimbria-fornix transection. $\mathbf{J}$ Neurosci. 12, 345-55.

Goodman, J. C., Van, M., Gopinath, S. P., Robertson, C. S., 2008. Pro-inflammatory and pro-apoptotic elements of the neuroinflammatory response are activated in traumatic brain injury. Acta Neurochir Suppl. 102, 437-9. 
Goodwill, M. H., Lawrence, D. A., Seegal, R. F., 2007. Polychlorinated biphenyls induce proinflammatory cytokine release and dopaminergic dysfunction: protection in interleukin-6 knockout mice. J Neuroimmunol. 183, 125-32.

Gordon, M. N., Schreier, W. A., Ou, X., Holcomb, L. A., Morgan, D. G., 1997. Exaggerated astrocyte reactivity after nigrostriatal deafferentation in the aged rat. J Comp Neurol. 388, 106-19.

Gorell, J. M., Peterson, E. L., Rybicki, B. A., Johnson, C. C., 2004. Multiple risk factors for Parkinson's disease. J Neurol Sci. 217, 169-74.

Gottlieb, M., Matute, C., 1999. Expression of nerve growth factor in astrocytes of the hippocampal CA1 area following transient forebrain ischemia. Neuroscience. 91, 1027-34.

Gourley, S. L., Kiraly, D. D., Howell, J. L., Olausson, P., Taylor, J. R., 2008. Acute hippocampal brain-derived neurotrophic factor restores motivational and forced swim performance after corticosterone. Biol Psychiatry. 64, 884-90.

Greenamyre, J. T., Betarbet, R., Sherer, T. B., 2003. The rotenone model of Parkinson's disease: genes, environment and mitochondria. Parkinsonism Relat Disord. 9 Suppl 2, S59-64.

Gribova, I. E., Gnedenko, B. B., Poleshchuk, V. V., Morozov, S. G., 2003. [Level of interferon-gamma, tumor necrosis factor alpha, and antibodies to them in blood serum from Parkinson disease patients]. Biomed Khim. 49, 208-12.

Groettrup, M., Khan, S., Schwarz, K., Schmidtke, G., 2001. Interferon-gamma inducible exchanges of 20 S proteasome active site subunits: why? Biochimie. 83, 367-372. 
Grondin, R., Zhang, Z., Yi, A., Cass, W. A., Maswood, N., Andersen, A. H., Elsberry, D. D., Klein, M. C., Gerhardt, G. A., Gash, D. M., 2002. Chronic, controlled GDNF infusion promotes structural and functional recovery in advanced parkinsonian monkeys. Brain. 125, 2191-201.

Guerini, F. R., Beghi, E., Riboldazzi, G., Zangaglia, R., Pianezzola, C., Bono, G., Casali, C., Di Lorenzo, C., Agliardi, C., Nappi, G., Clerici, M., Martignoni, E., 2009. BDNF Val66Met polymorphism is associated with cognitive impairment in Italian patients with Parkinson's disease. Eur J Neurol. 16, 1240-5.

Guillemin, G., Boussin, F. D., Le Grand, R., Croitoru, J., Coffigny, H., Dormont, D., 1996. Granulocyte macrophage colony stimulating factor stimulates in vitro proliferation of astrocytes derived from simian mature brains. Glia. 16, 71-80.

Gupta, S. P., Patel, S., Yadav, S., Singh, A. K., Singh, S., Singh, M. P., 2010. Involvement of Nitric Oxide in Maneb- and Paraquat-Induced Parkinson's Disease Phenotype in Mouse: Is There Any Link with Lipid Peroxidation? Neurochem Res.

Ha, Y., Park, H. S., Park, C. W., Yoon, S. H., Park, S. R., Hyun, D. K., Kim, E. Y., Park, H. C., 2005. Synthes Award for Resident Research on Spinal Cord and Spinal Column Injury: granulocyte macrophage colony stimulating factor (GM-CSF) prevents apoptosis and improves functional outcome in experimental spinal cord contusion injury. Clin Neurosurg. 52, 341-7.

Hald, A., Lotharius, J., 2005. Oxidative stress and inflammation in Parkinson's disease: is there a causal link? Experimental Neurology. 193, 279-290.

Hanisch, U. K., 2002. Microglia as a source and target of cytokines. Glia. 40, 140-55. 
Harling-Berg, C. J., Park, T. J., Knopf, P. M., 1999. Role of the cervical lymphatics in the Th2-type hierarchy of CNS immune regulation. J Neuroimmunol. 101, 111-27.

Hartlage-Rubsamen, M., Lemke, R., Schliebs, R., 1999. Interleukin-1beta, inducible nitric oxide synthase, and nuclear factor-kappaB are induced in morphologically distinct microglia after rat hippocampal lipopolysaccharide/interferon-gamma injection. J Neurosci Res. 57, 388-98.

Hartmann, A., Michel, P. P., Troadec, J. D., Mouatt-Prigent, A., Faucheux, B. A., Ruberg, M., Agid, Y., Hirsch, E. C., 2001. Is Bax a mitochondrial mediator in apoptotic death of dopaminergic neurons in Parkinson's disease? J Neurochem. $76,1785-93$.

Hartmann, A., Mouatt-Prigent, A., Faucheux, B. A., Agid, Y., Hirsch, E. C., 2002. FADD: A link between TNF family receptors and caspases in Parkinson's disease. Neurology. 58, 308-10.

Hartz, A. M., Bauer, B., Fricker, G., Miller, D. S., 2006. Rapid modulation of Pglycoprotein-mediated transport at the blood-brain barrier by tumor necrosis factor-alpha and lipopolysaccharide. Mol Pharmacol. 69, 462-470.

Hawkins, B. T., Davis, T. P., 2005. The blood-brain barrier/neurovascular unit in health and disease. Pharmacol Rev. 57, 173-85.

Hayashi, K., Ohta, S., Kawakami, Y., Toda, M., 2009. Activation of dendritic-like cells and neural stem/progenitor cells in injured spinal cord by GM-CSF. Neurosci Res. 64, 96-103. 
Hayashi, M., Dorf, M. E., Abromson-Leeman, S., 1993. Granulocyte-macrophage colony stimulating factor inhibits class II major histocompatibility complex expression and antigen presentation by microglia. J Neuroimmunol. 48, 23-32.

Hayley, S., Anisman, H., 2005. Multiple mechanisms of cytokine action in neurodegenerative and psychiatric states: neurochemical and molecular substrates. Curr Pharm Des. 11, 947-62.

Hayley, S., Brebner, K., Lacosta, S., Merali, Z., Anisman, H., 1999. Sensitization to the effects of tumor necrosis factor-alpha: neuroendocrine, central monoamine, and behavioral variations. J Neurosci. 19, 5654-65.

Hayley, S., Crocker, S. J., Smith, P., Shree, T., Park, D. S., 2004a. Mice lacking the tumor necrosis factor- $\alpha$ or Fas receptors are resistant to MPTP induced death of dopaminergic neurons. J Neurosci. 24, 2045-2053.

Hayley, S., Crocker, S. J., Smith, P. D., Shree, T., Jackson-Lewis, V., Przedborski, S., Mount, M., Slack, R., Anisman, H., Park, D. S., 2004b. Regulation of dopaminergic loss by Fas in a 1-methyl-4-phenyl-1,2,3,6-tetrahydropyridine model of Parkinson's disease. The Journal of neuroscience : the official journal of the Society for Neuroscience. 24, 2045-2053.

Hayley, S., Lacosta, S., Merali, Z., Van Rooijen, N., Anisman, H., 2001. Central Monoamine and Plasma Corticosterone Changes Induced by a Bacterial Endotoxin: Sensitization and Cross-sensitization Effects. Eur J Neurosci. 13, $1155-1165$. 
Heldt, S. A., Stanek, L., Chhatwal, J. P., Ressler, K. J., 2007. Hippocampus-specific deletion of BDNF in adult mice impairs spatial memory and extinction of aversive memories. Mol Psychiatry. 12, 656-70.

Hercus, T. R., Thomas, D., Guthridge, M. A., Ekert, P. G., King-Scott, J., Parker, M. W., Lopez, A. F., 2009. The granulocyte-macrophage colony-stimulating factor receptor: linking its structure to cell signaling and its role in disease. Blood. 114, 1289-98.

Herrera, A. J., Tomas-Camardiel, M., Venero, J. L., Cano, J., Machado, A., 2005. Inflammatory process as a determinant factor for the degeneration of substantia nigra dopaminergic neurons. Journal of neural transmission (Vienna, Austria : 1996). 112, 111-119.

Hirsch, E., Graybiel, A. M., Agid, Y. A., 1988. Melanized dopaminergic neurons are differentially susceptible to degeneration in Parkinson's disease. Nature. 334, 3458.

Hirsch, E. C., Hunot, S., 2009. Neuroinflammation in Parkinson's disease: a target for neuroprotection? Lancet Neurol. 8, 382-97.

Hirsch, E. C., Hunot, S., Hartmann, A., 2005. Neuroinflammatory processes in Parkinson's disease. Parkinsonism \& related disorders. 11 Suppl 1, S9-S15.

Ho, A., Blum, M., 1998. Induction of interleukin-1 associated with compensatory dopaminergic sprouting in the denervated striatum of young mice: model of aging and neurodegenerative disease. J Neurosci. 18, 5614-29.

Hoglinger, G. U., Feger, J., Prigent, A., Michel, P. P., Parain, K., Champy, P., Ruberg, M., Oertel, W. H., Hirsch, E. C., 2003. Chronic systemic complex I inhibition 
induces a hypokinetic multisystem degeneration in rats. J Neurochem. 84, 491502.

Hooper, D. C., Scott, G. S., Zborek, A., Mikheeva, T., Kean, R. B., Koprowski, H., Spitsin, S. V., 2000. Uric acid, a peroxynitrite scavenger, inhibits CNS inflammation, blood-CNS barrier permeability changes, and tissue damage in a mouse model of multiple sclerosis. FASEB J. 14, 691-8.

Howells, D. W., Porritt, M. J., Wong, J. Y., Batchelor, P. E., Kalnins, R., Hughes, A. J., Donnan, G. A., 2000. Reduced BDNF mRNA expression in the Parkinson's disease substantia nigra. Exp Neurol. 166, 127-35.

Huang, C. F., Li, G., Ma, R., Sun, S. G., Chen, J. G., 2008. Thrombin-induced microglial activation contributes to the degeneration of nigral dopaminergic neurons in vivo. Neurosci Bull. 24, 66-72.

Hung, H. C., Lee, E. H., 1996. The mesolimbic dopaminergic pathway is more resistant than the nigrostriatal dopaminergic pathway to MPTP and MPP+ toxicity: role of BDNF gene expression. Brain Res Mol Brain Res. 41, 14-26

Huang, R., Ke, W., Liu, Y., Wu, D., Feng, L., Jiang, C., Pei, Y., Gene therapy using lactoferrin-modified nanoparticles in a rotenone-induced chronic Parkinson model. J Neurol Sci. 290, 123-30.

Huang, X., Choi, J. K., Park, S. R., Ha, Y., Park, H., Yoon, S. H., Park, H. C., Park, J. O., Choi, B. H., 2007. GM-CSF inhibits apoptosis of neural cells via regulating the expression of apoptosis-related proteins. Neurosci Res. 58, 50-7.

Huang, X., Kim, J. M., Kong, T. H., Park, S. R., Ha, Y., Kim, M. H., Park, H., Yoon, S. H., Park, H. C., Park, J. O., Min, B. H., Choi, B. H., 2009. GM-CSF inhibits glial 
scar formation and shows long-term protective effect after spinal cord injury. $\mathbf{J}$ Neurol Sci. 277, 87-97.

Hulse, R. E., Kunkler, P. E., Fedynshyn, J. P., Kraig, R. P., 2004. Optimization of multiplexed bead-based cytokine immunoassays for rat serum and brain tissue. $\mathrm{J}$ Neurosci Methods. 136, 87-98.

Hung, H. C., Lee, E. H., 1996. The mesolimbic dopaminergic pathway is more resistant than the nigrostriatal dopaminergic pathway to MPTP and MPP+ toxicity: role of BDNF gene expression. Brain Res Mol Brain Res. 41, 14-26.

Hunot, S., Boissiere, F., Faucheux, B., Brugg, B., Mouatt-Prigent, A., Agid, Y., Hirsch, E. C., 1996. Nitric oxide synthase and neuronal vulnerability in Parkinson's disease. Neuroscience. $72,355-363$.

Hunot, S., Dugas, N., Faucheux, B., Hartmann, A., Tardieu, M., Debre, P., Agid, Y., Dugas, B., Hirsch, E. C., 1999. FcepsilonRII/CD23 is expressed in Parkinson's disease and induces, in vitro, production of nitric oxide and tumor necrosis factoralpha in glial cells. J Neurosci. 19, 3440-7.

Hunot, S., Vila, M., Teismann, P., Davis, R. J., Hirsch, E. C., Przedborski, S., Rakic, P., Flavell, R. A., 2004. JNK-mediated induction of cyclooxygenase 2 is required for neurodegeneration in a mouse model of Parkinson's disease. Proceedings of the National Academy of Sciences of the United States of America. 101, 665-670.

Iczkiewicz, J., Jackson, M. J., Smith, L. A., Rose, S., Jenner, P., 2006. Osteopontin expression in substantia nigra in MPTP-treated primates and in Parkinson's disease. Brain Res. 1118, 239-50. 
Iczkiewicz, J., Rose, S., Jenner, P., 2005. Increased osteopontin expression following intranigral lipopolysaccharide injection in the rat. Eur J Neurosci. 21, 1911-20.

Iravani, M. M., Leung, C. C., Sadeghian, M., Haddon, C. O., Rose, S., Jenner, P., 2005. The acute and the long-term effects of nigral lipopolysaccharide administration on dopaminergic dysfunction and glial cell activation. The European journal of neuroscience. 22, 317-330.

Ischiropoulos, H., Beckman, J. S., 2003. Oxidative stress and nitration in neurodegeneration: cause, effect, or association? J Clin Invest. 111, 163-9.

Jang, H., Boltz, D. A., Webster, R. G., Smeyne, R. J., 2008. Viral parkinsonism. Biochim Biophys Acta.

Jenner, P., 1998. Oxidative mechanisms in nigral cell death in Parkinson's disease. Mov Disord. 13 Suppl 1, 24-34.

Jeohn, G. H., Kim, W. G., Hong, J. S., 2000. Time dependency of the action of nitric oxide in lipopolysaccharide-interferon-gamma-induced neuronal cell death in murine primary neuron-glia co-cultures. Brain Res. 880, $173-7$.

Jin, F., Wu, Q., Lu, Y. F., Gong, Q. H., Shi, J. S., 2008. Neuroprotective effect of resveratrol on 6-OHDA-induced Parkinson's disease in rats. Eur J Pharmacol. $600,78-82$.

Jokinen, P., Bruck, A., Aalto, S., Forsback, S., Parkkola, R., Rinne, J. O., 2009. Impaired cognitive performance in Parkinson's disease is related to caudate dopaminergic hypofunction and hippocampal atrophy. Parkinsonism Relat Disord. 15, 88-93. 
Juillerat-Jeanneret, L., Schmitt, F., 2007. Chemical modification of therapeutic drugs or drug vector systems to achieve targeted therapy: looking for the grail. Med Res Rev. 27, 574-90.

Juliano, R. L., Ling, V., 1976. A surface glycoprotein modulating drug permeability in Chinese hamster ovary cell mutants. Biochim Biophys Acta. 455, 152-62.

Jung, J. S., Kim, D. H., Kim, H. S., Ginsenoside Rh1 suppresses inducible nitric oxide synthase gene expression in IFN-gamma-stimulated microglia via modulation of JAK/STAT and ERK signaling pathways. Biochem Biophys Res Commun.

Kakar, R., Kautz, B., Eklund, E. A., 2005. JAK2 is necessary and sufficient for interferon-gamma-induced transcription of the gene encoding gp91PHOX. J Leukoc Biol. 77, 120-7.

Kassiotis, G., Kollias, G., 2001. Uncoupling the proinflammatory from the immunosuppressive properties of tumor necrosis factor (TNF) at the p55 TNF receptor level: implications for pathogenesis and therapy of autoimmune demyelination. J Exp Med. 193, 427-34.

Kawada, H., Takizawa, S., Takanashi, T., Morita, Y., Fujita, J., Fukuda, K., Takagi, S., Okano, H., Ando, K., Hotta, T., 2006. Administration of hematopoietic cytokines in the subacute phase after cerebral infarction is effective for functional recovery facilitating proliferation of intrinsic neural stem/progenitor cells and transition of bone marrow-derived neuronal cells. Circulation. 113, 701-10.

Kawahara, K., Hosoya, R., Sato, H., Tanaka, M., Nakajima, T., Iwabuchi, S., 2002. Selective blockade of astrocytic glutamate transporter GLT-1 with dihydrokainate 
prevents neuronal death during ouabain treatment of astrocyte/neuron cocultures. Glia. 40, 337-49.

Kawanokuchi, J., Mizuno, T., Takeuchi, H., Kato, H., Wang, J., Mitsuma, N., Suzumura, A., 2006. Production of interferon-gamma by microglia. Mult Scler. 12, 558-64.

Kean, R. B., Spitsin, S. V., Mikheeva, T., Scott, G. S., Hooper, D. C., 2000. The peroxynitrite scavenger uric acid prevents inflammatory cell invasion into the central nervous system in experimental allergic encephalomyelitis through maintenance of blood-central nervous system barrier integrity. J Immunol. 165, $6511-8$.

Kearns, C. M., Cass, W. A., Smoot, K., Kryscio, R., Gash, D. M., 1997. GDNF protection against 6-OHDA: time dependence and requirement for protein synthesis. J Neurosci. 17, 7111-8.

Kearns, C. M., Gash, D. M., 1995. GDNF protects nigral dopamine neurons against 6hydroxydopamine in vivo. Brain Res. 672, 104-11.

Kelada, S. N., Checkoway, H., Kardia, S. L., Carlson, C. S., Costa-Mallen, P., Eaton, D. L., Firestone, J., Powers, K. M., Swanson, P. D., Franklin, G. M., Longstreth, W. T., Jr., Weller, T. S., Afsharinejad, Z., Costa, L. G., 2006. 5' and 3' region variability in the dopamine transporter gene (SLC6A3), pesticide exposure and Parkinson's disease risk: a hypothesis-generating study. Hum Mol Genet. 15, $3055-62$.

Kim, J. K., Choi, B. H., Park, H. C., Park, S. R., Kim, Y. S., Yoon, S. H., Park, H. S., Kim, E. Y., Ha, Y., 2004. Effects of GM-CSF on the neural progenitor cells. Neuroreport. 15, 2161-5. 
Kim, N. K., Choi, B. H., Huang, X., Snyder, B. J., Bukhari, S., Kong, T. H., Park, H., Park, H. C., Park, S. R., Ha, Y., 2009a. Granulocyte-macrophage colonystimulating factor promotes survival of dopaminergic neurons in the 1-methyl-4phenyl-1,2,3,6-tetrahydropyridine-induced murine Parkinson's disease model. Eur J Neurosci. 29, 891-900.

Kim, W. G., Mohney, R. P., Wilson, B., Jeohn, G. H., Liu, B., Hong, J. S., 2000. Regional difference in susceptibility to lipopolysaccharide-induced neurotoxicity in the rat brain: role of microglia. The Journal of neuroscience : the official journal of the Society for Neuroscience. 20, 6309-6316.

Kim, Y. J., Shin, J. I., Park, K. W., Lee, H. Y., Kang, H. J., Koo, B. K., Park, B. J., Sohn, D. W., Oh, B. H., Park, Y. B., Kim, H. S., 2009b. The effect of granulocytecolony stimulating factor on endothelial function in patients with myocardial infarction. Heart. 95, 1320-5.

Kirik, D., Georgievska, B., Bjorklund, A., 2004. Localized striatal delivery of GDNF as a treatment for Parkinson disease. Nat Neurosci. 7, 105-10.

Klein, C., Lohmann-Hedrich, K., 2007. Impact of recent genetic findings in Parkinson's disease. Curr Opin Neurol. 20, 453-64.

Klein, J. B., Rane, M. J., Scherzer, J. A., Coxon, P. Y., Kettritz, R., Mathiesen, J. M., Buridi, A., McLeish, K. R., 2000. Granulocyte-macrophage colony-stimulating factor delays neutrophil constitutive apoptosis through phosphoinositide 3-kinase and extracellular signal-regulated kinase pathways. J Immunol. 164, 4286-91. 
Klintworth, H., Newhouse, K., Li, T., Choi, W. S., Faigle, R., Xia, Z., 2007. Activation of c-Jun N-terminal protein kinase is a common mechanism underlying paraquatand rotenone-induced dopaminergic cell apoptosis. Toxicol Sci. 97, 149-62.

Knott, C., Stern, G., Wilkin, G. P., 2000. Inflammatory regulators in Parkinson's disease: iNOS, lipocortin-1, and cyclooxygenases-1 and -2. Mol Cell Neurosci. 16, 72439.

Koizumi, S., Fujishita, K., 2007. [Gliotransmitter ATP-mediated cell-to-cell communication]. Brain Nerve. 59, 707-15.

Kong, T., Choi, J. K., Park, H., Choi, B. H., Snyder, B. J., Bukhari, S., Kim, N. K., Huang, X., Park, S. R., Park, H. C., Ha, Y., 2009. Reduction in programmed cell death and improvement in functional outcome of transient focal cerebral ischemia after administration of granulocyte-macrophage colony-stimulating factor in rats. Laboratory investigation. J Neurosurg. 111, 155-63.

Konsman, J. P., Tridon, V., Dantzer, R., 2000. Diffusion and action of intracerebroventricularly injected interleukin-1 in the CNS. Neuroscience. 101, 957-67.

Koprich, J. B., Reske-Nielsen, C., Mithal, P., Isacson, O., 2008. Neuroinflammation mediated by IL-1beta increases susceptibility of dopamine neurons to degeneration in an animal model of Parkinson's disease. J Neuroinflammation. 5, 8.

Kordower, J. H., Palfi, S., Chen, E. Y., Ma, S. Y., Sendera, T., Cochran, E. J., Mufson, E. J., Penn, R., Goetz, C. G., Comella, C. D., 1999. Clinicopathological findings 
following intraventricular glial-derived neurotrophic factor treatment in a patient with Parkinson's disease. Ann Neurol. 46, 419-24.

Kotone-Miyahara, Y., Yamashita, K., Lee, K. K., Yonehara, S., Uchiyama, T., Sasada, M., Takahashi, A., 2004. Short-term delay of Fas-stimulated apoptosis by GMCSF as a result of temporary suppression of FADD recruitment in neutrophils: evidence implicating phosphatidylinositol 3-kinase and MEK1-ERK1/2 pathways downstream of classical protein kinase C. J Leukoc Biol. 76, 1047-56.

Koustilieri, E., Sopper, S., Scheller, C., ter Meulen, V., Riederer, P., 2002. Parkinsonism in HIV dementia. J Neural Transm. 109, 767-75.

Kraft, A. D., McPherson, C. A., Harry, G. J., 2009. Heterogeneity of microglia and TNF signaling as determinants for neuronal death or survival. Neurotoxicology. 30 , 785-93.

Kreutzberg, G. W., 1996. Microglia: a sensor for pathological events in the CNS. Trends Neurosci. 19, 312-8.

Krum, J. M., Phillips, T. M., Rosenstein, J. M., 2002. Changes in astroglial GLT-1 expression after neural transplantation or stab wounds. Exp Neurol. 174, 137-49.

Kuan, Y. H., Lin, R. H., Tsao, L. T., Lin, C. N., Wang, J. P., 2005. Artocarpol A stimulation of superoxide anion generation in neutrophils involved the activation of PLC, PKC and p38 mitogen-activated PK signaling pathways. Br J Pharmacol. $145,460-8$

Kuhnke, D., Jedlitschky, G., Grube, M., Krohn, M., Jucker, M., Mosyagin, I., Cascorbi, I., Walker, L. C., Kroemer, H. K., Warzok, R. W., Vogelgesang, S., 2007. MDR1P-Glycoprotein (ABCB1) Mediates Transport of Alzheimer's amyloid-beta 
peptides--implications for the mechanisms of Abeta clearance at the blood-brain barrier. Brain Pathol. 17, 347-53.

Kumar, S., Jack, R., 2006. Origin of monocytes and their differentiation to macrophages and dendritic cells. J Endotoxin Res. 12, 278-84.

Kurkowska-Jastrzebska, I., Litwin, T., Joniec, I., Ciesielska, A., Przybylkowski, A., Czlonkowski, A., Czlonkowska, A., 2004. Dexamethasone protects against dopaminergic neurons damage in a mouse model of Parkinson's disease. Int Immunopharmacol. 4, 1307-18.

Kurkowska-Jastrzebska, I., Wronska, A., Kohutnicka, M., Czlonkowski, A., Czlonkowska, A., 1999a. The inflammatory reaction following 1-methyl-4phenyl-1,2,3, 6-tetrahydropyridine intoxication in mouse. Experimental Neurology. 156, 50-61.

Kurkowska-Jastrzebska, I., Wronska, A., Kohutnicka, M., Czlonkowski, A., Czlonkowska, A., 1999b. MHC class II positive microglia and lymphocytic infiltration are present in the substantia nigra and striatum and in mouse model of Parkinson's disease. Acta Neurobiol Exp. 59, 1-8.

L' Episcopo, F., Tirolo, C., Testa, N., Caniglia, S., Morale, M. C., Marchetti, B., 2010. Glia as a turning point in the therapeutic strategy of Parkinson's disease. CNS Neurol Disord Drug Targets. 9, 349-72.

Lafortune, L., Nalbantoglu, J., Antel, J. P., 1996. Expression of tumor necrosis factor alpha (TNF alpha) and interleukin 6 (IL-6) mRNA in adult human astrocytes: comparison with adult microglia and fetal astrocytes. J Neuropathol Exp Neurol. $55,515-21$. 
Landrigan, P. J., Sonawane, B., Butler, R. N., Trasande, L., Callan, R., Droller, D., 2005. Early environmental origins of neurodegenerative disease in later life. Environmental health perspectives. 113, 1230-1233.

Lang, A. E., Gill, S., Patel, N. K., Lozano, A., Nutt, J. G., Penn, R., Brooks, D. J., Hotton, G., Moro, E., Heywood, P., Brodsky, M. A., Burchiel, K., Kelly, P., Dalvi, A., Scott, B., Stacy, M., Turner, D., Wooten, V. G., Elias, W. J., Laws, E. R., Dhawan, V., Stoessl, A. J., Matcham, J., Coffey, R. J., Traub, M., 2006. Randomized controlled trial of intraputamenal glial cell line-derived neurotrophic factor infusion in Parkinson disease. Ann Neurol. 59, 459-66.

Langston, J. W., 2006. Parkinson's complex: parkinsonism is just the tip of the iceberg. Ann Neurol. 59, 591-6.

Langston, J. W., Forno, L. S., Tetrud, J., Reeves, A. G., Kaplan, J. A., Karluk, D., 1999. Evidence of active nerve cell degeneration in the substantia nigra of humans years after 1-methyl-4-phenyl-1,2,3,6-tetrahydropyridine exposure. Ann Neurol. 46, $598-605$.

Laurie, C., Reynolds, A., Coskun, O., Bowman, E., Gendelman, H. E., Mosley, R. L., 2007. CD4+ $T$ cells from Copolymer-1 immunized mice protect dopaminergic neurons in the 1-methy-4-phenyl-1,2,3,6-tetrahydropyridine model of Parkinson's disease. J Neuroimmunol. 183, 60-68.

Le, W., Rowe, D., Xie, W., Ortiz, I., He, Y., Appel, S. H., 2001. Microglial activation and dopaminergic cell injury: an in vitro model relevant to Parkinson's disease. The Journal of neuroscience : the official journal of the Society for Neuroscience. $21,8447-8455$. 
Lee da, Y., Oh, Y. J., Jin, B. K., 2005. Thrombin-activated microglia contribute to death of dopaminergic neurons in rat mesencephalic cultures: dual roles of mitogenactivated protein kinase signaling pathways. Glia. 51, 98-110.

Lehnardt, S., Massillon, L., Follett, P., Jensen, F. E., Ratan, R., Rosenberg, P. A., Volpe, J. J., Vartanian, T., 2003. Activation of innate immunity in the CNS triggers neurodegeneration through a Toll-like receptor 4-dependent pathway. Proceedings of the National Academy of Sciences of the United States of America. 100, 85148519.

Levivier, M., Przedborski, S., Bencsics, C., Kang, U. J., 1995. Intrastriatal implantation of fibroblasts genetically engineered to produce brain-derived neurotrophic factor prevents degeneration of dopaminergic neurons in a rat model of Parkinson's disease. J Neurosci. 15, 7810-20.

Li, X., Yin, J., Cheng, C. M., Sun, J. L., Li, Z., Wu, Y. L., 2005. Paraquat induces selective dopaminergic nigrostriatal degeneration in aging C57BL/6 mice. Chin Med J (Engl). 118, 1357-61.

Liberatore, G. T., Jackson-Lewis, V., Vukosavic, 1999. Inducible nitric oxide synthase stimulates dopaminergic neurodegeneration in the MPTP model of Parkinson's disese. Nature medicine. 5, 1403-1439.

Lin, L. F., Doherty, D. H., Lile, J. D., Bektesh, S., Collins, F., 1993. GDNF: a glial cell line-derived neurotrophic factor for midbrain dopaminergic neurons. Science. $260,1130-2$. 
Ling, Z., Chang, Q. A., Tong, C. W., Leurgans, S. E., Lipton, J. W., Carvey, P. M., 2004a. Rotenone potentiates dopamine neuron loss in animals exposed to lipopolysaccharide prenatally. Experimental Neurology. 190, 373-383.

Ling, Z., Gayle, D. A., Ma, S. Y., Lipton, J. W., Tong, C. W., Hong, J. S., Carvey, P. M., 2002. In utero bacterial endotoxin exposure causes loss of tyrosine hydroxylase neurons in the postnatal rat midbrain. Mov Disord. 17, 116-24.

Ling, Z., Zhu, Y., Tong, C. W., Snyder, J. A., Carvey, P. M., 2006. Progressive dopamine neurons loss following supra-nigral lipopolysaccharide (LPS) infusion into rats exposed to LPS prenatally. Exp Neurol. 24.

Ling, Z. D., Chang, Q., Lipton, J. W., Tong, C. W., Landers, T. M., Carvey, P. M., 2004b. Combined toxicity of prenatal bacterial endotoxin exposure and postnatal 6-hydroxydopamine in the adult rat midbrain. Neuroscience. 124, 619-628.

Liou, H. H., Tsai, M. C., Chen, C. J., Jeng, J. S., Chang, Y. C., Chen, S. Y., Chen, R. C., 1997. Environmental risk factors and Parkinson's disease: a case-control study in Taiwan. Neurology. 48, 1583-8.

Litteljohn, D., Mangano, E., Shukla, N., Hayley, S., 2009. Interferon-gamma deficiency modifies the motor and co-morbid behavioral pathology and neurochemical changes provoked by the pesticide paraquat. Neuroscience. 164, 1894-906.

Litteljohn, D., Mangano, E. N., Hayley, S., 2008. Cyclooxygenase-2 deficiency modifies the neurochemical effects, motor impairment and co-morbid anxiety provoked by paraquat administration in mice. Eur J Neurosci. 28, 707-16. 
Liu, B., Gao, H. M., Hong, J. S., 2003. Parkinson's disease and exposure to infectious agents and pesticides and the occurrence of brain injuries: role of neuroinflammation. Environ Health Perspect. 111, 1065-1073.

Liva, S. M., Kahn, M. A., Dopp, J. M., de Vellis, J., 1999. Signal transduction pathways induced by GM-CSF in microglia: significance in the control of proliferation. Glia. 26, 344-52.

Livak, K. J., Schmittgen, T. D., 2001. Analysis of relative gene expression data using real-time quantitative PCR and the 2(-Delta Delta C(T)) Method. Methods. 25, $402-8$.

Logroscino, G., 2005. The role of early life environmental risk factors in Parkinson disease: what is the evidence? Environmental health perspectives. 113, 12341238.

Love, S., Plaha, P., Patel, N. K., Hotton, G. R., Brooks, D. J., Gill, S. S., 2005. Glial cell line-derived neurotrophic factor induces neuronal sprouting in human brain. Nat Med. 11, 703-4.

Lucas, P. C., McAllister-Lucas, L. M., Nunez, G., 2004. NF-kappaB signaling in lymphocytes: a new cast of characters. J Cell Sci. 117, 31-9.

Madrigal, J. L., Garcia-Bueno, B., Caso, J. R., Perez-Nievas, B. G., Leza, J. C., 2006. Stress-induced oxidative changes in brain. CNS Neurol Disord Drug Targets. 5, $561-8$

Mak, S. K., McCormack, A. L., Manning-Bog, A. B., Cuervo, A. M., Di Monte, D. A., 2010. Lysosomal degradation of alpha-synuclein in vivo. J Biol Chem. 285, $13621-9$. 
Malipiero, U. V., Frei, K., Fontana, A., 1990. Production of hemopoietic colonystimulating factors by astrocytes. J Immunol. 144, 3816-21.

Man, S., Ubogu, E. E., Ransohoff, R. M., 2007. Inflammatory cell migration into the central nervous system: a few new twists on an old tale. Brain Pathol. 17, 243-50.

Mana, P., Linares, D., Fordham, S., Staykova, M., Willenborg, D., 2006. Deleterious role of IFNgamma in a toxic model of central nervous system demyelination. Am J Pathol. 168, 1464-73.

Mandel, S., Grunblatt, E., Youdim, M., 2000a. cDNA microarray to study gene expression of dopaminergic neurodegeneration and neuroprotection in MPTP and 6-hydroxydopamine models: implications for idiopathic Parkinson's disease. Journal of neural transmission. Supplementum. (60), 117-124.

Mandel, S., Grunblatt, E., Youdim, M., 2000b. cDNA microarray to study gene expression of dopaminergic neurodegeneration and neuroprotection in MPTP and 6-hydroxydopamine models: implications for idiopathic Parkinson's disease. J Neural Transm Suppl. 117-24.

Mander, P., Brown, G. C., 2005. Activation of microglial NADPH oxidase is synergistic with glial iNOS expression in inducing neuronal death: a dual-key mechanism of inflammatory neurodegeneration. J.Neuroinflammation. 2, 20.

Mangano, E. N., Hayley, S., 2009. Inflammatory priming of the substantia nigra influences the impact of later paraquat exposure: Neuroimmune sensitization of neurodegeneration. Neurobiol Aging. 30, 1361-78.

Manning-Bog, A. B., McCormack, A. L., Li, J., Uversky, V. N., Fink, A. L., Di Monte, D. A., 2002. The herbicide paraquat causes up-regulation and aggregation of 
alpha-synuclein in mice: paraquat and alpha-synuclein. Journal of Biological Chemistry. 277, 1641-1644.

Manning-Bog, A. B., McCormack, A. L., Purisai, M. G., Bolin, L. M., Di Monte, D. A., 2003. Alpha-synuclein overexpression protects against paraquat-induced neurodegeneration. The Journal of neuroscience : the official journal of the Society for Neuroscience. 23, 3095-3099.

Marques, L., Brucet, M., Lloberas, J., Celada, A., 2004. STAT1 regulates lipopolysaccharide and TNF-alpha-dependent expression of transport associated with antigen processing 1 and low molecular mass polypeptide 2 genes in macrophages by distinct mechanisms. J Immunol. 173, 1103-110.

Marsden, C. D., Linazasoro, G., Obeso, J. A., 1997. An introduction to the new surgery for Parkinson's disease. Past and present problems. Adv Neurol. 74, 143-7.

Marsden, C. D., Parkes, J. D., 1977. Success and problems of long-term levodopa therapy in Parkinson's disease. Lancet. 1, 345-9.

Martin, S., Gee, J. R., Bruce-Keller, A. J., Keller, J. N., 2004. Loss of an individual proteasome subunit alters motor function but not cognitive function or ambulation in mice. Neurosci Lett. 357, 76-78.

Masaki, T., Matsushita, S., Arai, H., Takeda, A., Itoyama, Y., Mochizuki, H., Kamakura, K., Ohara, S., Higuchi, S., 2003. Association between a polymorphism of brainderived neurotrophic factor gene and sporadic Parkinson's disease. Ann Neurol. $54,276-7$

Maswood, N., Grondin, R., Zhang, Z., Stanford, J. A., Surgener, S. P., Gash, D. M., Gerhardt, G. A., 2002. Effects of chronic intraputamenal infusion of glial cell 
line-derived neurotrophic factor (GDNF) in aged Rhesus monkeys. Neurobiol Aging. 23, 881-9.

Mattson, M. P., 2005. NF-kappaB in the survival and plasticity of neurons. Neurochem Res. 30, 883-93.

Matzinger, P., 2002. The danger model: a renewed sense of self. Science. 296, 301-5.

McCormack, A. L., Atienza, J. G., Johnston, L. C., Andersen, J. K., Vu, S., Di Monte, D. A., 2005. Role of oxidative stress in paraquat-induced dopaminergic cell degeneration. Journal of neurochemistry. 93, 1030-1037.

McCormack, A. L., Di Monte, D. A., 2003. Effects of L-dopa and other amino acids against paraquat-induced nigrostriatal degeneration. Journal of neurochemistry. $85,82-86$.

McCormack, A. L., Thiruchelvam, M., Manning-Bog, A. B., Thiffault, C., Langston, J. W., Cory-Slechta, D. A., Di Monte, D. A., 2002. Enviromental risk factors and Parkinson's disease: selective degeneration of nigral dopaminergic neurons caused by the herbicide paraquat. Neurobiol Dis. 10, 119-127.

McCoy, M. K., Ruhn, K. A., Martinez, T. N., McAlpine, F. E., Blesch, A., Tansey, M. G., 2008. Intranigral lentiviral delivery of dominant-negative TNF attenuates neurodegeneration and behavioral deficits in hemiparkinsonian rats. Mol Ther. 16, $1572-9$.

McCoy, M. K., Tansey, M. G., 2008. TNF signaling inhibition in the CNS: implications for normal brain function and neurodegenerative disease. J Neuroinflammation. 5, 45. 
McGeer, E. G., Klegeris, A., McGeer, P. L., 2005. Inflammation, the complement system and the diseases of aging. Neurobiol Aging. 26 Suppl 1, 94-7.

McGeer, P. L., Itagaki, S., Boyes, B. E., McGeer, E. G., 1988. Reactive microglia are positive for HLA-DR in the substantia nigra of Parkinson's and Alzheimer's disease brains. Neurology. 38, 1285-91.

McGeer, P. L., Schwab, C., Parent, A., Doudet, D., 2003. Presence of reactive microglia in monkey substantia nigra years after 1-methyl-4-phenyl-1,2,3,6tetrahydropyridine administration. Annals of Neurology. 54, 599-604.

McKinnon, P. J., Margolskee, R. F., 1996. SC1: a marker for astrocytes in the adult rodent brain is upregulated during reactive astrocytosis. Brain Res. 709, 27-36.

McLay, R. N., Freeman, S. M., Zadina, J. E., 2001. Administration of FGF-1 through transfected cells alleviates MPTP toxicity in mice. Neurotox Res. 3, 249-253.

McLay, R. N., Kimura, M., Banks, W. A., Kastin, A. J., 1997. Granulocyte-macrophage colony-stimulating factor crosses the blood--brain and blood--spinal cord barriers. Brain. 120 ( Pt 11), 2083-91.

McNaught, K. S., Belizaire, R., Jenner, P., Olanow, C. W., Isacson, O., 2002. Selective loss of $20 \mathrm{~S}$ proteasome alpha-subunits in the substantia nigra pars compacta in Parkinson's disease. Neurosci Lett. 326, 155-158.

Meller, R., Stevens, S. L., Minami, M., Cameron, J. A., King, S., Rosenzweig, H., Doyle, K., Lessov, N. S., Simon, R. P., Stenzel-Poore, M. P., 2005. Neuroprotection by osteopontin in stroke. J Cereb Blood Flow Metab. 25, 217-25.

Michelucci, A., Heurtaux, T., Grandbarbe, L., Morga, E., Heuschling, P., 2009. Characterization of the microglial phenotype under specific pro-inflammatory and 
anti-inflammatory conditions: Effects of oligomeric and fibrillar amyloid-beta. J Neuroimmunol. 210, 3-12.

Miklossy, J., Doudet, D. D., Schwab, C., Yu, S., McGeer, E. G., McGeer, P. L., 2006. Role of ICAM-1 in persisting inflammation in Parkinson disease and MPTP monkeys. Exp Neurol. 197, 275-83.

Miller, R. L., James-Kracke, M., Sun, G. Y., Sun, A. Y., 2009. Oxidative and inflammatory pathways in Parkinson's disease. Neurochem Res. 34, 55-65.

Mishto, M., Bellavista, E., Santoro, A., Stolzing, A., Ligorio, C., Ohm, T., Grune, T., Franceschi, C., 2006. Immunoproteasome and LMP2 polymorphism in aged and Alzheimer's disease brains. Neurobiol Aging. 27, 54-66.

Mizuno, T., Zhang, G., Takeuchi, H., Kawanokuchi, J., Wang, J., Sonobe, Y., Jin, S., Takada, N., Komatsu, Y., Suzumura, A., 2008. Interferon-gamma directly induces neurotoxicity through a neuron specific, calcium-permeable complex of IFNgamma receptor and AMPA GluR1 receptor. FASEB J. 22, 1797-806.

Mladenovic, A., Perovic, M., Raicevic, N., Kanazir, S., Rakic, L., Ruzdijic, S., 2004. 6Hydroxydopamine increases the level of TNFalpha and bax mRNA in the striatum and induces apoptosis of dopaminergic neurons in hemiparkinsonian rats. Brain research. 996, 237-245.

Mogi, M., Harada, M., Narabayashi, H., Inagaki, H., Minami, M., Nagatsu, T., 1996. Interleukin (IL)-1beta, IL-2, IL-4, IL-6 and transforming growth factor-alpha levels are elevated in ventricular cerebrospinal fluid in juvenile parkinsonism and Parkinson's disease. Neurosci Lett. 211, 13-16. 
Mogi, M., Harada, M., Riederer, P., Narabayashi, H., Fuitia, K., Nagatsu, T., 1994. Tumor necrosis factor-alpha (TNF- $\alpha)$ increases both in the brain and in the cerebrospinal fluid from parkinsonian patients. Neurosci Lett. 165, 208-10.

Mogi, M., Kondo, T., Mizuno, Y., Nagatsu, T., 2007. p53 protein, interferon-gamma, and NF-kappaB levels are elevated in the parkinsonian brain. Neurosci Lett. 414, 947.

Mogi, M., Togari, A., Kondo, T., Mizuno, Y., Kogure, O., Kuno, S., Ichinose, H., Nagatsu, T., 2001. Glial cell line-derived neurotrophic factor in the substantia nigra from control and parkinsonian brains. Neurosci Lett. 300, 179-81.

Mogi, M., Togari, A., Kondo, T., Mizuno, Y., Komure, O., Kuno, S., Ichinose, H., Nagatsu, T., 1999. Brain-derived growth factor and nerve growth factor concentrations are decreased in the substantia nigra in Parkinson's disease. Neurosci Lett. 270, 45-8.

Montenegro, D. E., Franklin, T., Moscinski, L. C., Zuckerman, K. S., Hu, X. T., 2009. TGFbeta inhibits GM-CSF-induced phosphorylation of ERK and MEK in human myeloid leukaemia cell lines via inhibition of phosphatidylinositol 3-kinase (PI3k). Cell Prolif. 42, 1-9.

Montero-Menei, C. N., Sindji, L., Garcion, E., Mege, M., Couez, D., Gamelin, E., Darcy, F., 1996. Early events of the inflammaroty reaction induced in rat brain by lipopolysaccharide intracerebral injection: relative contribution of peripheral monocytes and activated microglia. Brain Res. 724, 55-66. 
Montine, T. J., Milatovic, D., Gupta, R. C., Valyi-Nagy, T., Morrow, J. D., Breyer, R. M., 2002. Neuronal oxidative damage from activated innate immunity is EP2 receptor-dependent. J Neurochem. 83, 463-470.

Moran, L. B., Duke, D. C., Graeber, M. B., 2007. The microglial gene regulatory network activated by interferon-gamma. J Neuroimmunol. 183, 1-6.

Morris, H. R., 2005. Genetics of Parkinson's disease. Ann Med. 37, 86-96.

Mosley, R. L., Benner, E. J., Kadiu, I., Thomas, M., Boska, M. D., Hasan, K., Laurie, C., Gendelman, H. E., 2006. Neuroinflammation, Oxidative Stress and the Pathogenesis of Parkinson's Disease. Clin Neurosci Res. 6, 261-281.

Mounayar, S., Boulet, S., Tande, D., Jan, C., Pessiglione, M., Hirsch, E. C., Feger, J., Savasta, M., Francois, C., Tremblay, L., 2007. A new model to study compensatory mechanisms in MPTP-treated monkeys exhibiting recovery. Brain. $130,2898-914$.

Mount, M. P., Lira, A., Grimes, D., Smith, P. D., Faucher, S., Slack, R., Anisman, H., Hayley, S., Park, D. S., 2007. Involvement of interferon-gamma in microglialmediated loss of dopaminergic neurons. J Neurosci. 27, 3328-37.

Murer, M. G., Yan, Q., Raisman-Vozari, R., 2001. Brain-derived neurotrophic factor in the control human brain, and in Alzheimer's disease and Parkinson's disease. Prog Neurobiol. 63, 71-124.

Murray, P. J., 2007. The JAK-STAT signaling pathway: input and output integration. J Immunol. 178, 2623-9.

Nagatsu, T., Mogi, M., Ichinose, H., Togari, A., 2000. Changes in cytokine and neurotrophinsin Parkinson's disease. J Neural Transm Suppl. 60, 277-290. 
Nagatsu, T., Sawada, M., 2005. Inflammatory process in Parkinson's disease: role for cytokines. Current pharmaceutical design. 11, 999-1016.

Nagatsu, T., Sawada, M., 2007. Biochemistry of postmortem brains in Parkinson's disease: historical overview and future prospects. J Neural Transm Suppl. 113-20.

Nakagawa, T., Suga, S., Kawase, T., Toda, M., 2006. Intracarotid injection of granulocyte-macrophage colony-stimulating factor induces neuroprotection in a rat transient middle cerebral artery occlusion model. Brain Res. 1089, 179-85.

Nakamura, A., Kitami, T., Mori, H., Mizuno, Y., Hattori, N., 2006. Nuclear localization of the 20S proteasome subunit in Parkinson's disease. Neurosci Lett. 406, 43-48.

Neumann, H., Misgeld, T., Matsumuro, K., Wekerle, H., 1998. Neurotrophins inhibit major histocompatibility class II inducibility of microglia: involvement of the p75 neurotrophin receptor. Proc Natl Acad Sci U S A. 95, 5779-84.

Newhouse, K., Hsuan, S. L., Chang, S. H., Cai, B., Wang, Y., Xia, Z., 2004. Rotenoneinduced apoptosis is mediated by p38 and JNK MAP kinases in human dopaminergic SH-SY5Y cells. Toxicol Sci. 79, 137-46.

Nicklas, W. J., Vyas, I., Heikkila, R. E., 1985. Inhibition of NADH-linked oxidation in brain mitochondria by 1-methyl-4-phenyl-pyridine, a metabolite of the neurotoxin, 1-methyl-4-phenyl-1,2,5,6-tetrahydropyridine. Life Sci. 36, 2503-8.

Nimmerjahn, A., Kirchhoff, F., Helmchen, F., 2005. Resting microglial cells are highly dynamic surveillants of brain parenchyma in vivo. Science. $308,1314-8$.

Niwa, M., Hotta, K., Kanamori, Y., Kumada, M., Hirota, M., Kozawa, O., Fujimoto, S., 2004. p38 MAPK associated with stereoselective priming by grepafloxacin on O2- production in neutrophils. Free Radic Biol Med. 36, 1259-69. 
Nutt, J. G., Burchiel, K. J., Comella, C. L., Jankovic, J., Lang, A. E., Laws, E. R., Jr., Lozano, A. M., Penn, R. D., Simpson, R. K., Jr., Stacy, M., Wooten, G. F., 2003. Randomized, double-blind trial of glial cell line-derived neurotrophic factor (GDNF) in PD. Neurology. 60, 69-73.

Offen, D., Beart, P. M., Cheung, N. S., Pascoe, C. J., Hochman, A., Gorodin, S., Melamed, E., Bernard, R., Bernard, O., 1998. Transgenic mice expressing human Bcl-2 in their neurons are resistant to 6-hydroxydopamine and 1-methyl-4-phenyl1,2,3,6- tetrahydropyridine neurotoxicity. Proc Natl Acad Sci U S A. 95, 5789-94.

Ogata, A., Tashiro, K., Nukuzuma, S., Nagashima, K., Hall, W. W., 1997. A rat model of Parkinson's disease induced by Japanese encephalitis virus. J Neurovirol. 3, 1417.

Okuno, T., Nakatsuji, Y., Kumanogoh, A., Moriya, M., Ichinose, H., Sumi, H., Fujimura, H., Kikutani, H., Sakoda, S., 2005. Loss of dopaminergic neurons by the induction of inducible nitric oxide synthase and cyclooxygenase-2 via CD 40: relevance to Parkinson's disease. J Neurosci Res. 81, 874-82.

Ossowska, K., Wardas, J., Kuter, K., Nowak, P., Dabrowska, J., Bortel, A., Labus, L., Kwiecinski, A., Krygowska-Wajs, A., Wolfarth, S., 2005a. Influence of paraquat on dopaminergic transporter in the rat brain. Pharmacol Rep. 57, 330-5.

Ossowska, K., Wardas, J., Smialowska, M., Kunter, K., Lenda, T., Wieronska, J. M., Zieba, B., Nowak, P., Dabrowska, J., Bortel, A., Kwiecinski, A., Wolfarth, S., 2005b. A slowly devoloping dysfunction of dopaminergic nigrostriatal neurons induced by long-term paraquat administration in rats: an animal model of preclinical stages of Parkinson's disease? Eur J Neurosci. 22, 1294-1304. 
Pahan, K., Sheikh, F. G., Liu, X., Hilger, S., McKinney, M., Petro, T. M., 2001. Induction of nitric-oxide synthase and activation of NF-kappaB by interleukin-12 p40 in microglial cells. J Biol Chem. 276, 7899-905.

Paik, J., Lee, J. Y., Hwang, D., 2002. Signaling pathways for TNFa-induced COX-2 expression: mediation through MAP kinases and NFkB, and inhibition by certain nonsteroidal anti-inflammatory drugs. Adv Exp Med Biol. 507, 503-8.

Parain, K., Murer, M. G., Yan, Q., Faucheux, B., Agid, Y., Hirsch, E., Raisman-Vozari, R., 1999. Reduced expression of brain-derived neurotrophic factor protein in Parkinson's disease substantia nigra. Neuroreport. 10, 557-61.

Pardridge, W. M., 2002. Targeting neurotherapeutic agents through the blood-brain barrier. Arch Neurol. 59, 35-40.

Patel, N. K., Bunnage, M., Plaha, P., Svendsen, C. N., Heywood, P., Gill, S. S., 2005. Intraputamenal infusion of glial cell line-derived neurotrophic factor in PD: a two-year outcome study. Ann Neurol. 57, 298-302.

Pawate, S., Shen, Q., Fan, F., Bhat, N. R., 2004. Redox regulation of glial inflammatory response to lipopolysaccharide and interferongamma. Journal of neuroscience research. $77,540-551$.

Peleshok, J., Saragovi, H. U., 2006. Functional mimetics of neurotrophins and their receptors. Biochem Soc Trans. 34, 612-7.

Peng, G. S., Li, G., Tzeng, N. S., Chen, P. S., Chuang, D. M., Hsu, Y. D., Yang, S., Hong, J. S., 2005a. Valproate pretreatment protects dopaminergic neurons from LPS-induced neurotoxicity in rat primary midbrain cultures: role of microglia. Brain Res Mol Brain Res. 134, 162-9. 
Peng, J., Mao, X. O., Stevenson, F. F., Hsu, M., Andersen, J. K., 2004. The herbicide paraquat induces dopaminergic nigral apoptosis through sustained activation of the JNK pathway. Journal of Biological Chemistry. 279, 32626-32632.

Peng, J., Oo, M. L., Andersen, J. K., 2010. Synergistic effects of environmental risk factors and gene mutations in Parkinson's disease accelerate age-related neurodegeneration. J Neurochem 115, 1363-73.

Peng, J., Peng, L., Stevenson, F. F., Doctrow, S. R., Andersen, J. K., 2007. Iron and paraquat as synergistic environmental risk factors in sporadic Parkinson's disease accelerate age-related neurodegeneration. J Neurosci. 27, 6914-22.

Peng, J., Stevenson, F. F., Doctrow, S. R., Andersen, J. K., 2005b. Superoxide dismutase/catalase mimetics are neuroprotective against selective paraquatmediated dopaminergic neuron death in the substantia nigra: implications for Parkinson disease. J Biol Chem. 280, 29194-29198.

Peterson, A. L., Nutt, J. G., 2008. Treatment of Parkinson's disease with trophic factors. Neurotherapeutics. 5, 270-80.

Phares, T. W., Fabis, M. J., Brimer, C. M., Kean, R. B., Hooper, D. C., 2007. A peroxynitrite-dependent pathway is responsible for blood-brain barrier permeability changes during a central nervous system inflammatory response: TNF-alpha is neither necessary nor sufficient. J Immunol. 178, 7334-43.

Planas, A. M., Gorina, R., Chamorro, A., 2006. Signalling pathways mediating inflammatory responses in brain ischaemia. Biochem Soc Trans. 34, 1267-70.

Plata-Salaman, C. R., 1994. Meal patterns in response to the intracerebroventricular administration of interleukin-1 beta in rats. Physiol Behav. 55, 727-33. 
Popovich, P. G., Longbrake, E. E., 2008. Can the immune system be harnessed to repair the CNS? Nat Rev Neurosci. 9, 481-93.

Porritt, M. J., Batchelor, P. E., Howells, D. W., 2005. Inhibiting BDNF expression by antisense oligonucleotide infusion causes loss of nigral dopaminergic neurons. Exp Neurol. 192, 226-34.

Pott Godoy, M. C., Ferrari, C. C., Pitossi, F. J., Nigral neurodegeneration triggered by striatal AdIL-1 administration can be exacerbated by systemic IL-1 expression. J Neuroimmunol. 222, 29-39.

Pott Godoy, M. C., Tarelli, R., Ferrari, C. C., Sarchi, M. I., Pitossi, F. J., 2008. Central and systemic IL-1 exacerbates neurodegeneration and motor symptoms in a model of Parkinson's disease. Brain. 131, 1880-94.

Poynter, M. E., Cloots, R., van Woerkom, T., Butnor, K. J., Vacek, P., Taatjes, D. J., Irvin, C. G., Janssen-Heininger, Y. M., 2004. NF-kappa B activation in airways modulates allergic inflammation but not hyperresponsiveness. J Immunol. 173, 7003-9.

Prasad, K., Tarasewicz, E., Mathew, J., Strickland, P. A., Buckley, B., Richardson, J. R., Richfield, E. K., 2009. Toxicokinetics and toxicodynamics of paraquat accumulation in mouse brain. Exp Neurol. 215, 358-67.

Prasad, K., Winnik, B., Thiruchelvam, M., Buckley, B., Mirochnitchenko, O., Richfield, E. K., 2007. Prolonged Toxicokinetics and Toxicodynamics of Paraquat in Mouse Brain. Environ Health Perspect. 115, 1448-1453.

Priyadarshi, A., Khuder, S. A., Schaub, E. A., Priyadarshi, S. S., 2001. Environmental risk factors and Parkinson's disease: a metaanalysis. Environ Res. 86, 122-7. 
Przedborski, S., Jackson-Lewis, V., Yokoyama, R., Shibata, T., Dawson, V. L., Dawson, T. M., 1996. Role of neuronal nitric oxide in 1-methyl-4-phenyl-1,2,3,6tetrahydropyridine (MPTP)-induced dopaminergic neurotoxicity. Proceedings of the National Academy of Sciences of the United States of America. 93, 45654571.

Purisai, M. G., McCormack, A. L., Cumine, S., Li, J., Isla, M. Z., Di Monte, D. A., 2007. Microglial activation as a priming event leading to paraquat-induced dopaminergic cell degeneration. Neurobiol Dis. 25, 392-400.

Pyo, C. W., Lee, S. H., Choi, S. Y., 2008. Oxidative stress induces PKR-dependent apoptosis via IFN-gamma activation signaling in Jurkat $\mathrm{T}$ cells. Biochem Biophys Res Commun. 377, 1001-6.

Qian, L., Block, M. L., Wei, S. J., Lin, C. F., Reece, J., Pang, H., Wilson, B., Hong, J. S., Flood, P. M., 2006. Interleukin-10 protects lipopolysaccharide-induced neurotoxicity in primary midbrain cultures by inhibiting the function of NADPH oxidase. J Pharmacol Exp Ther. 319, 44-52.

Qian, L., Gao, X., Pei, Z., Wu, X., Block, M., Wilson, B., Hong, J. S., Flood, P. M., 2007. NADPH oxidase inhibitor DPI is neuroprotective at femtomolar concentrations through inhibition of microglia over-activation. Parkinsonism Relat Disord. 13 Suppl 3, S316-20.

Qian, L., Hu, X., Zhang, D., Snyder, A., Wu, H. M., Li, Y., Wilson, B., Lu, R. B., Hong, J. S., Flood, P. M., 2009. beta2 Adrenergic receptor activation induces microglial NADPH oxidase activation and dopaminergic neurotoxicity through an ERKdependent/protein kinase A-independent pathway. Glia. 57, 1600-9. 
Qian, L., Wei, S. J., Zhang, D., Hu, X., Xu, Z., Wilson, B., El-Benna, J., Hong, J. S., Flood, P. M., 2008. Potent anti-inflammatory and neuroprotective effects of TGFbetal are mediated through the inhibition of ERK and p47phox-Ser345 phosphorylation and translocation in microglia. J Immunol. 181, 660-8.

Qin, L., Liu, Y., Wang, T., Wei, S. J., Block, M. L., Wilson, B., Liu, B., Hong, J. S., 2004. NADPH oxidase mediates lipopolysaccharide-induced neurotoxicity and proinflammatory gene expression in activated microglia. J Biol Chem. 279, 141521.

Quintero, E. M., Willis, L., Singleton, R., Harris, N., Huang, P., Bhat, N., Granholm, A. C., 2006. Behavioral and morphological effects of minocycline in the 6hydroxydopamine rat model of Parkinson's disease. Brain Res. 1093, 198-207.

Radad, K., Moldzio, R., Rausch, W. D., 2010. Minocycline protects dopaminergic neurons against long-term rotenone toxicity. Can J Neurol Sci. 37, 81-5.

Ramachandiran, S., Hansen, J. M., Jones, D. P., Richardson, J. R., Miller, G. W., 2007. Divergent Mechanisms of Paraquat, MPP+, and Rotenone Toxicity: Oxidation of Thioredoxin and Caspase-3 Activation. Toxicological Sciences. 95, 163.

Re, D. B., Przedborski, S., 2006. Fractalkine: moving from chemotaxis to neuroprotection. Nat Neurosci. 9, 859-61.

Re, F., Belyanskaya, S. L., Riese, R. J., Cipriani, B., Fischer, F. R., Granucci, F., Ricciardi-Castagnoli, P., Brosnan, C., Stern, L. J., Strominger, J. L., Santambrogio, L., 2002. Granulocyte-macrophage colony-stimulating factor induces an expression program in neonatal microglia that primes them for antigen presentation. J Immunol. 169, 2264-73. 
Reale, M., Greig, N. H., Kamal, M. A., 2009. Peripheral chemo-cytokine profiles in Alzheimer's and Parkinson's diseases. Mini Rev Med Chem. 9, 1229-41.

Reddy, P. H., Manczak, M., Zhao, W., Nakamura, K., Bebbington, C., Yarranton, G., Mao, P., 2009. Granulocyte-macrophage colony-stimulating factor antibody suppresses microglial activity: implications for anti-inflammatory effects in Alzheimer's disease and multiple sclerosis. J Neurochem. 111, 1514-28.

Reynolds, A. D., Stone, D. K., Mosley, R. L., Gendelman, H. E., 2009. Nitrated \{alpha\}synuclein-induced alterations in microglial immunity are regulated by CD4+ $\mathrm{T}$ cell subsets. J Immunol. 182, 4137-49.

Richardson, J. R., Quan, Y., Sherer, T. B., Greenamyre, J. T., Miller, G. W., 2005. Paraquat neurotoxicity is distinct from that of MPTP and rotenone. Toxicological sciences : an official journal of the Society of Toxicology. 88, 193-201.

Ritz, B., Yu, F., 2000. Parkinson's disease mortality and pesticide exposure in California 1984-1994. Int J Epidemiol. 29, 323-9.

Rivest, S., 2009. Regulation of innate immune responses in the brain. Nat Rev Immunol. 9, 429-39.

Robertson, D. C., Schmidt, O., Ninkina, N., Jones, P. A., Sharkey, J., Buchman, V. L., 2004. Developmental loss and resistance to MPTP toxicity of dopaminergic neurones in substantia nigra pars compacta of gamma-synuclein, alpha-synuclein and double alpha/gamma-synuclein null mutant mice. J Neurochem. 89, 1126-36.

Rochet, J. C., Outeiro, T. F., Conway, K. A., Ding, T. T., Volles, M. J., Lashuel, H. A., Bieganski, R. M., Lindquist, S. L., Lansbury, P. T., 2004. Interactions among alpha-synuclein, dopamine, and biomembranes: some clues for understanding 
neurodegeneration in Parkinson's disease. Journal of Molecular Neuroscience : JMN. 23, 23-34.

Rodriguez-Oroz, M. C., Obeso, J. A., Lang, A. E., Houeto, J. L., Pollak, P., Rehncrona, S., Kulisevsky, J., Albanese, A., Volkmann, J., Hariz, M. I., Quinn, N. P., Speelman, J. D., Guridi, J., Zamarbide, I., Gironell, A., Molet, J., Pascual-Sedano, B., Pidoux, B., Bonnet, A. M., Agid, Y., Xie, J., Benabid, A. L., Lozano, A. M., Saint-Cyr, J., Romito, L., Contarino, M. F., Scerrati, M., Fraix, V., Van Blercom, N., 2005. Bilateral deep brain stimulation in Parkinson's disease: a multicentre study with 4 years follow-up. Brain. 128, 2240-9.

Rodriguez-Pallares, J., Parga, J. A., Munoz, A., Rey, P., Guerra, M. J., LabandeiraGarcia, J. L., 2007. Mechanism of 6-hydroxydopamine neurotoxicity: the role of NADPH oxidase and microglial activation in 6-hydroxydopamine-induced degeneration of dopaminergic neurons. J Neurochem. 103, 145-56.

Rogers, R. J., Monnier, J. M., Nick, H. S., 2001. Tumor necrosis factor-alpha selectively induces MnSOD expression via mitochondria-to-nucleus signaling, whereas interleukin-1 beta utilizes an alternative pathway. J Biol Chem. 276, 20419-27.

Rosenzweig, H. L., Lessov, N. S., Henshall, D. C., Minami, M., Simon, R. P., StenzelPoore, M. P., 2004. Endotoxin preconditioning prevents cellular inflammatory response during ischemic neuroprotection in mice. Stroke. 35, 2576-2581.

Rossini, M., Cheunsuchon, B., Donnert, E., Ma, L. J., Thomas, J. W., Neilson, E. G., Fogo, A. B., 2005. Immunolocalization of fibroblast growth factor-1 (FGF-1), its receptor (FGFR-1), and fibroblast-specific protein-1 (FSP-1) in inflammatory renal disease. Kidney Int. 68, 2621-2628. 
Rothwell, N. J., Hopkins, S. J., 1995. Cytokines and the nervous system II: Actions and mechanisms of action. Trends Neurosci. 18, 130-6.

Rousselet, E., Callebert, J., Parain, K., Joubert, C., Hunot, S., Hartmann, A., Jacque, C., Perez-Diaz, F., Cohen-Salmon, C., Launay, J. M., Hirsch, E. C., 2002. Role of TNF-a receptors in mice intoxicated with the parkinsonian toxin MPTP. Exp Neurol. 177, 183-192.

Saint-Pierre, M., Tremblay, M. E., Sik, A., Gross, R. E., Cicchetti, F., 2006. Temporal effects of paraquat/maneb on microglia activation and dopamine neuronal loss in older rats. J Biol Chem. 98, 760-772.

Sakai, I., and Kraft, A.S., 1997. The kinase domain of Jak2 mediates induction of bcl-2 and delays cell death in hematopoietic cells. J Biol Chem. 272, 12350-8.

Sakamoto, K. M., Mignacca, R. C., Gasson, J. C., 1994. Signal transduction by granulocyte-macrophage colony-stimulating factor and interleukin-3 receptors. Receptors Channels. 2, 175-81.

Sakon, S., Xue, X., Takekawa, M., Sasazuki, T., Okazaki, T., Kojima, Y., Piao, J. H., Yagita, H., Okumura, K., Doi, T., Nakano, H., 2003. NF-kappaB inhibits TNFinduced accumulation of ROS that mediate prolonged MAPK activation and necrotic cell death. EMBO J. 22, 3898-909.

Salach, J. I., Singer, T. P., Castagnoli, N., Jr., Trevor, A., 1984. Oxidation of the neurotoxic amine 1-methyl-4-phenyl-1,2,3,6-tetrahydropyridine (MPTP) by monoamine oxidases A and B and suicide inactivation of the enzymes by MPTP. Biochemical and biophysical research communications. 125, 831-835. 
Salehi, Z., Mashayekhi, F., 2009. Brain-derived neurotrophic factor concentrations in the cerebrospinal fluid of patients with Parkinson's disease. J Clin Neurosci. 16, 90-3.

Salkeni, M. A., Lynch, J. L., Otamis-Price, T., Banks, W. A., 2009. Lipopolysaccharide impairs blood-brain barrier P-glycoprotein function in mice through prostaglandin- and nitric oxide-independent pathways. J Neuroimmune Pharmacol. 4, 276-82.

Salvatore, M. F., Ai, Y., Fischer, B., Zhang, A. M., Grondin, R. C., Zhang, Z., Gerhardt, G. A., Gash, D. M., 2006. Point source concentration of GDNF may explain failure of phase II clinical trial. Exp Neurol. 202, 497-505.

Santambrogio, L., Belyanskaya, S. L., Fischer, F. R., Cipriani, B., Brosnan, C. F., Ricciardi-Castagnoli, P., Stern, L. J., Strominger, J. L., Riese, R., 2001. Developmental plasticity of CNS microglia. Proc Natl Acad Sci U S A. 98, 6295300.

Saporito, M. S., Brown, E. M., Miller, M. S., Carswell, S., 1999. CEP-1347/KT-7515, an inhibitor of c-jun N-terminal kinase activation, attenuates the 1-methyl-4-phenyl tetrahydropyridine-mediated loss of nigrostriatal dopaminergic neurons In vivo. J Pharmacol Exp Ther. 288, 421-7.

Saporito, M. S., Thomas, B. A., Scott, R. W., 2000. MPTP activates c-Jun NH(2)terminal kinase $(\mathrm{JNK})$ and its upstream regulatory kinase MKK4 in nigrostriatal neurons in vivo. J Neurochem. $75,1200-8$.

Saura, J., Pares, M., Bove, J., Pezzi, S., Alberch, J., Marin, C., Tolosa, E., Marti, M. J., 2003. Intranigral infusion of interleukin-lb activates astrocytes and protects from subsequent 6-hydroxydopamine neurotoxicity. J Neurochem. 85, 651-61. 
Scatton, B., Rouquier, L., Javoy-Agid, F., Agid, Y., 1982. Dopamine deficiency in the cerebral cortex in Parkinson disease. Neurology. 32, 1039-40.

Schabitz, W. R., Kruger, C., Pitzer, C., Weber, D., Laage, R., Gassler, N., Aronowski, J., Mier, W., Kirsch, F., Dittgen, T., Bach, A., Sommer, C., Schneider, A., 2008. A neuroprotective function for the hematopoietic protein granulocyte-macrophage colony stimulating factor (GM-CSF). J Cereb Blood Flow Metab. 28, 29-43.

Schermer, C., Humpel, C., 2002. Granulocyte macrophage-colony stimulating factor activates microglia in rat cortex organotypic brain slices. Neurosci Lett. 328, 180 4.

Schmidt, E. D., Janszen, A. W., Wouterlood, F. G., Tilders, F. J., 1995. Interleukin-1induced long-lasting changes in hypothalamic corticotropin-releasing hormone (CRH)--neurons and hyperresponsiveness of the hypothalamus-pituitary-adrenal axis. J Neurosci. 15, 7417-26.

Schmidt, H. D., Duman, R. S., 2007. The role of neurotrophic factors in adult hippocampal neurogenesis, antidepressant treatments and animal models of depressive-like behavior. Behav Pharmacol. 18, 391-418.

Schmidt, H. D., Duman, R. S., 2010. Peripheral BDNF Produces Antidepressant-Like Effects in Cellular and Behavioral Models. Neuropsychopharmacology.

Schmittgen, T. D., Livak, K. J., 2008. Analyzing real-time PCR data by the comparative C(T) method. Nat Protoc. 3, 1101-8.

Schroder, K., Hertzog, P. J., Ravasi, T., Hume, D. A., 2004. Interferon-gamma: an overview of signals, mechanisms and functions. J Leukoc Biol. 75, 163-89. 
Schulz-Schaeffer, W. J., 2010. The synaptic pathology of alpha-synuclein aggregation in dementia with Lewy bodies, Parkinson's disease and Parkinson's disease dementia. Acta Neuropathol. 120, 131-43.

Seroogy, K. B., Lundgren, K. H., Tran, T. M., Guthrie, K. M., Isackson, P. J., Gall, C. M., 1994. Dopaminergic neurons in rat ventral midbrain express brain-derived neurotrophic factor and neurotrophin-3 mRNAs. J Comp Neurol. 342, 321-34.

Sherer, T. B., Betarbet, R., Greenamyre, J. T., 2002a. Environment, mitochondria, and Parkinson's disease. Neuroscientist. 8, 192-7.

Sherer, T. B., Betarbet, R., Kim, J. H., Greenamyre, J. T., 2003a. Selective microglial activation in the rat rotenone model of Parkinson's disease. Neurosci Lett. 341, 87-90.

Sherer, T. B., Betarbet, R., Stout, A. K., Lund, S., Baptista, M., Panov, A. V., Cookson, M. R., Greenamyre, J. T., 2002b. An in vitro model of Parkinson's disease: linking mitochondrial impairment to altered alpha-synuclein metabolism and oxidative damage. J Neurosci. 22, 7006-15.

Sherer, T. B., Betarbet, R., Testa, C. M., Seo, B. B., Richardson, J. R., Kim, J. H., Miller, G. W., Yagi, T., Matsuno-Yagi, A., Greenamyre, J. T., 2003b. Mechanism of toxicity in rotenone models of Parkinson's disease. The Journal of neuroscience : the official journal of the Society for Neuroscience. 23, 10756-10764.

Sherer, T. B., Richardson, J. R., Testa, C. M., Seo, B. B., Panov, A. V., Yagi, T., Matsuno-Yagi, A., Miller, G. W., Greenamyre, J. T., 2007. Mechanism of toxicity of pesticides acting at complex I: relevance to environmental etiologies of Parkinson's disease. J Neurochem. 100, 1469-79. 
Shibata, H., Katsuki, H., Nishiwaki, M., Kume, T., Kaneko, S., Akaike, A., 2003. Lipopolysaccharide-induced dopaminergic cell death in rat midbrain silce cultures: role of inducible nitric oxide synthase and protection by indomethacin. $\mathrm{J}$ Neurochem. 86, 1201-1212.

Shie, F. S., Montine, K.S., Breyer, R.M., Montine, T.J.,, 2005. Microglial EP2 is critical to neurotoxicity from activated cerebral innate immunity. Glia. 52, 70-7.

Shimizu, K., Matsubara, K., Ohtaki, K., Fujimaru, S., Saito, O., Shiono, H., 2003. Paraquat induces long-lasting dopamine overflow through the excitotoxic pathway in the striatum of freely moving rats. Brain research. 976, 243-252.

Shimizu, K., Ohtaki, K., Matsubara, K., Aoyama, K., Uezono, T., Saito, O., Suno, M., Ogawa, K., Hayase, N., Kimura, K., Shiono, H., 2001. Carrier-mediated processes in blood-brain barrier penetration and neural uptake of paraquat. Brain Res. 906, $135-142$.

Shopova, V. L., Dancheva, V. Y., Salovsky, P. T., Stoyanova, A. M., 2009. Protective effects of a superoxide dismutase/catalase mimetic compound against paraquat pneumotoxicity in rat lung. Respirology. 14, 504-10.

Shults, C. W., Kimber, T., Altar, C. A., 1995. BDNF attenuates the effects of intrastriatal injection of 6-hydroxydopamine. Neuroreport. 6, 1109-12.

Sindhu, K. M., Saravanan, K. S., Mohanakumar, K. P., 2005. Behavioral differences in a rotenone-induced hemiparkinsonian rat model developed following intranigral or median forebrain bundle infusion. Brain research. 1051, 25-34.

Slevin, J. T., Gerhardt, G. A., Smith, C. D., Gash, D. M., Kryscio, R., Young, B., 2005. Improvement of bilateral motor functions in patients with Parkinson disease 
through the unilateral intraputaminal infusion of glial cell line-derived neurotrophic factor. J Neurosurg. 102, 216-22.

Smith, W. W., Pei, Z., Jiang, H., Moore, D. J., Liang, Y., West, A. B., Dawson, V. L., Dawson, T. M., Ross, C. A., 2005. Leucine-rich repeat kinase 2 (LRRK2) interacts with parkin, and mutant LRRK2 induces neuronal degeneration. Proc Natl Acad Sci USA. 102, 18676-18681.

Somayajulu-Nitu, M., Sandhu, J. K., Cohen, J., Sikorska, M., Sridhar, T. S., Matei, A., Borowy-Borowski, H., Pandey, S., 2009. Paraquat induces oxidative stress, neuronal loss in substantia nigra region and parkinsonism in adult rats: neuroprotection and amelioration of symptoms by water-soluble formulation of coenzyme Q10. BMC Neurosci. 10,88.

Sorce, S., Krause, K. H., 2009. NOX enzymes in the central nervous system: from signaling to disease. Antioxid Redox Signal. 11, 2481-504.

Soulet, D., Rivest, S., 2008. Microglia. Curr Biol. 18, R506-8.

Sozzani, S., Bonecchi, R., D'Amico, G., Luini, W., Bernasconi, S., Allavena, P., Mantovani, A., 1998. Old and new chemokines. Pharmacological regulation of chemokine production and receptor expression: mini-review. J Chemother. 10, $142-5$.

Springer, T. A., 1994. Traffic signals for lymphocyte recirculation and leukocyte emigration: the multistep paradigm. Cell. 76, 301-14.

Sriram, K., Matheson, J. M., Benkovic, S. A., Miller, D. B., Luster, M. I., O'Callaghan, J. P., 2002. Mice deficient in TNF receptors are protected against dopaminergic neurotoxicity: implications for Parkinson's disease. The FASEB journal : official 
publication of the Federation of American Societies for Experimental Biology. 16, $1474-1476$.

Sriram, K., Matheson, J. M., Benkovic, S. A., Miller, D. B., Luster, M. I., O'Callaghan, J. P., 2006. Deficiency of TNF receptors suppresses microglial activation and alters the susceptibility of brain regions to MPTP-induced neurotoxicity: role of TNFalpha. FASEB J. 20, 670-82.

Streit, W. J., Conde, J. R., Fendrick, S. E., Flanary, B. E., Mariani, C. L., 2005. Role of microglia in the central nervous system's immune response. Neurol Res. 27, 68591.

Sugama, S., Takenouchi, T., Cho, B. P., Joh, T. H., Hashimoto, M., Kitani, H., 2009. Possible roles of microglial cells for neurotoxicity in clinical neurodegenerative diseases and experimental animal models. Inflamm Allergy Drug Targets. 8, 27784.

Sulahian, T. H., Hogger, P., Wahner, A. E., Wardwell, K., Goulding, N. J., Sorg, C., Droste, A., Stehling, M., Wallace, P. K., Morganelli, P. M., Guyre, P. M., 2000. Human monocytes express CD163, which is upregulated by IL-10 and identical to p155. Cytokine. 12, 1312-21.

Suntres, Z. E., 2002. Role of antioxidants in paraquat toxicity. Toxicology. 180, 65-77.

Takahashi, M., Yamada, T., 1999. Viral etiology for Parkinson's disease--a possible role of influenza A virus infection. Japanese journal of infectious diseases. 52, 89-98.

Takeuchi, H., Jin, S., Wang, J., Zhang, G., Kawanokuchi, J., Kuno, R., Sonobe, Y., Mizuno, T., Suzumura, A., 2006. Tumor necrosis factor- $\alpha$ induces neurotoxicity 
via glutamate release from hemichannels of activated microglia in an autocrine manner. J Biol. Chem. 281, 21362-8.

Tan, E. K., Skipper, L. M., 2007. Pathogenic mutations in Parkinson disease. Hum Mutat. $28,641-53$.

Tanaka, S. K., Sugiura, S., Matsuoka-Omura, E., Sasaki, T., Yagita, Y., Hori, M., 2004. Infiltrating macrophages as in vivo targets for intravenous gene delivery in cerebral infarction. Stroke. 35, 1968-1973.

Tanner, C. M., Goldman, S. M., 1996. Epidemiology of Parkinson's disease. Neurol Clin. $14,317-35$.

Tatton, N. A., 2000. Increased caspase 3 and Bax immunoreactivity accompany nuclear GAPDH translocation and neuronal apoptosis in Parkinson's disease. Exp Neurol. $166,29-43$.

Tatton, W. G., Chalmers-Redman, R., Brown, D., Tatton, N., 2003. Apoptosis in Parkinson's disease: signals for neuronal degradation. Ann Neurol. 53 Suppl 3, S61-70; discussion S70-2.

Teismann, P., Ferger, B., 2001. Inhibition of the cyclooxygenase isoenzymes COX-1 and COX-2 provide neuroprotection in the MPTP-mouse model of Parkinson's disease. Synapse. 39, 167-74.

Teismann, P., Tieu, K., Cohen, O., Choi, D. K., Wu du, C., Marks, D., Vila, M., JacksonLewis, V., Przedborski, S., 2003. Pathogenic role of glial cells in Parkinson's disease. Mov Disord. 18, 121-129.

Terasaki, T., Hosoya, K., 1999. The blood-brain barrier efflux transporters as a detoxifying system for the brain. Adv Drug Deliv Rev. 36, 195-209. 
Thiruchelvam, M., Brockel, B. J., Richfield, E. K., Baggs, R. B., Cory-Slechta, D. A., 2000a. Potentiated and preferential effects of combined paraquat and maneb on nigrostriatal dopamine systems: environmental risk factors for Parkinson's disease? Brain research. 873, 225-234.

Thiruchelvam, M., McCormack, A., Richfield, E. K., Baggs, R. B., Tank, A. W., Di Monte, D. A., Cory-Slechta, D. A., 2003. Age-related irreversible progressive nigrostriatal dopaminergic neurotoxicity in the paraquat and maneb model of the Parkinson's disease phenotype. Eur J Neurosci. 18, 589-600.

Thiruchelvam, M., Prokopenko, O., Cory-Slechta, D. A., Richfield, E. K., Buckley, B., Mirochnitchenko, O., 2005. Overexpression of superoxide dismutase or glutathione peroxidase protects against the paraquat + maneb-induced Parkinson disease phenotype. Journal of Biological Chemistry. 280, 22530-22539.

Thiruchelvam, M., Richfield, E. K., Baggs, R. B., Tank, A. W., Cory-Slechta, D. A., 2000b. The nigrostriatal dopaminergic system as a preferential target of repeated exposures to combined paraquat and maneb: implications for Parkinson's disease. The Journal of neuroscience : the official journal of the Society for Neuroscience. $20,9207-9214$.

Thomas, B., Beal, M. F., 2007. Parkinson's disease. Hum Mol Genet. 16 Spec No. 2, R183-94.

Thomas, T., Timmer, M., Cesnulevicius, K., Hitti, E., Kotlyarov, A., Gaestel, M., 2008. MAPKAP kinase 2-deficiency prevents neurons from cell death by reducing neuroinflammation--relevance in a mouse model of Parkinson's disease. J Neurochem. 105, 2039-52. 
Thomson, A. W., Lotze, M. T. Eds.), 2003. The Cytokine Handbook. Academic Press, London.

Thornberry, N. A., Bull, H. G., Calaycay, J. R., Chapman, K. T., Howard, A. D., Kostura, M. J., Miller, D. K., Molineaux, S. M., Weidner, J. R., Aunins, J., et al., 1992. A novel heterodimeric cysteine protease is required for interleukin-1 beta processing in monocytes. Nature. $356,768-74$.

Timmer, M., Cesnulevicius, K., Winkler, C., Kolb, J., Lipokatic-Takacs, E., Jungnickel, J., 2007. Fibroblast growth factor (FGF)-2 and FGF receptor 3 are required for the development of the substantia nigra, and FGF-2 plays a crucial role for the rescue of dopaminergic neurons after 6-hydroxydopamine lesion. J Neurosci. 27, 459-71.

Tomac, A., Lindqvist, E., Lin, L. F., Ogren, S. O., Young, D., Hoffer, B. J., Olson, L., 1995. Protection and repair of the nigrostriatal dopaminergic system by GDNF in vivo. Nature. $373,335-9$.

Toulouse, A., Sullivan, A. M., 2008. Progress in Parkinson's disease-where do we stand? Prog Neurobiol. 85, 376-92.

Tournier, C., Hess, P., Yang, D. D., Xu, J., Turner, T. K., Nimnual, A., Bar-Sagi, D., Jones, S. N., Flavell, R. A., Davis, R. J., 2000. Requirement of JNK for stressinduced activation of the cytochrome c-mediated death pathway. Science. 288, $870-4$

Tsatsanis, C., Androulidaki, A., Venihaki, M., Margioris, A. N., 2006. Signalling networks regulating cyclooxygenase-2. Int J Biochem Cell Biol. 38, 1654-61.

Twig, G., Graf, S. A., Messerli, M. A., Smith, P. J., Yoo, S. H., Shirihai, O. S., 2005. Synergistic amplification of beta-amyloid- and interferon-gamma-induced 
microglial neurotoxic response by the senile plaque component chromogranin $\mathrm{A}$. Am J Physiol Cell Physiol. 288, C169-75.

Uversky, V. N., Li, J., Bower, K., Fink, A. L., 2002. Synergistic effects of pesticides and metals on the fibrillation of alpha-synuclein: implications for Parkinson's disease. Neurotoxicology. 23, 527-36.

Van Kaer, L., Ashton-Rickardt, P. G., Eichelberger, M., Gaczynska, M., Nagashima, K., Rock, K. L., Goldberg, A. L., Doherty, P. C., Tonegawa, S., 1994. Altered peptidase and viral-specific $\mathrm{T}$ cell response in LMP2 mutant mice. Immunity. 1, 533-41.

Vijitruth, R., Liu, M., Choi, D. Y., Nguyen, X. V., Hunter, R. L., Bing, G., 2006. Cyclooxygenase-2 mediates microglial activation and secondary dopaminergic cell death in the mouse MPTP model of Parkinson's disease. J Neuroinflammation. 3, 6 .

Vila, M., Jackson-Lewis, V., Vukosavic, S., Djaldetti, R., Liberatore, G., Offen, D., Korsmeyer, S. J., Przedborski, S., 2001. Bax ablation prevents dopaminergic neurodegeneration in the 1-methyl- 4-phenyl-1,2,3,6-tetrahydropyridine mouse model of Parkinson's disease. Proc Natl Acad Sci U S A. 98, 2837-42.

Vila, M., Przedborski, S., 2003. Targeting programmed cell death in neurodegenerative diseases. Nat Rev Neurosci. 4, 365-75.

Villadangos, J. A., Schnorrer, P., Wilson, N. S., 2005. Control of MHC class II antigen presentation in dendritic cells: a balance between creative and destructive forces. Immunological reviews. 207, 191-205. 
von Boyen, G. B., Steinkamp, M., Geerling, I., Reinshagen, M., Schafer, K. H., Adler, G., Kirsch, J., 2006. Proinflammatory cytokines induce neurotrophic factor expression in enteric glia: a key to the regulation of epithelial apoptosis in Crohn's disease. Inflamm Bowel Dis. 12, 346-54.

Waetzig, V., Czeloth, K., Hidding, U., Mielke, K., Kanzow, M., Brecht, S., Goetz, M., Lucius, R., Herdegen, T., Hanisch, U. K., 2005. c-Jun N-terminal kinases (JNKs) mediate pro-inflammatory actions of microglia. Glia. 50, 235-246.

Walker, D. G., Terai, K., Matsuo, A., Beach, T. G., McGeer, E. G., McGeer, P. L., 1998. Immunohistochemical analyses of fibroblast growth factor receptor-1 in the human sustantia nigra. Comparison between normal and Parkinson's disease cases. Brain Res. 794, 181-187.

Wang, J., Bankiewicz, K. S., Plunkett, R. J., Oldfield, E. H., 1994. Intrastriatal implantation of interleukin-1. Reduction of parkinsonism in rats by enhancing neuronal sprouting from residual dopaminergic neurons in the ventral tegmental area of the midbrain. J Neurosurg. 80, 484-90.

Wang, V., Chia, L. G., Ni, D. R., Cheng, L. J., Ho, Y. P., Cheng, F. C., Hong, J. S., 2004. Effects of the combined treatment of naloxone and indomethacin on catecholamines and behavior after intranigral lipopolysaccharide injection. Neurochem Res. 29, 341-6.

Wang, X., Suzuki, Y., 2007. Microglia produce IFN-gamma independently from T cells during acute toxoplasmosis in the brain. J Interferon Cytokine Res. 27, 599-605. 
Wang, Y., Zhang, Y., Wei, Z., Li, H., Zhou, H., Zhang, Z., 2009. JNK inhibitor protects dopaminergic neurons by reducing COX-2 expression in the MPTP mouse model of subacute Parkinson's disease. J Neurol Sci. 285, 172-7.

Watabe, M., Nakaki, T., 2008. Mitochondrial complex I inhibitor rotenone inhibits and redistributes vesicular monoamine transporter 2 via nitration in human dopaminergic SH-SY5Y cells. Mol Pharmacol. 74, 933-40.

Weisskopf, M. G., Weuve, J., Nie, H., Saint-Hilaire, M. H., Sudarsky, L., Simon, D. K., Hersh, B., Schwartz, J., Wright, R. O., Hu, H., Association of Cumulative Lead Exposure with Parkinson's Disease. Environ Health Perspect.

Weninger, W., von Andrian, U. H., 2003. Chemokine regulation of naive T cell traffic in health and disease. Semin Immunol. 15, 257-70.

Westerlund, M., Belin, A. C., Anvret, A., Hakansson, A., Nissbrandt, H., Lind, C., Sydow, O., Olson, L., Galter, D., 2009. Association of a polymorphism in the ABCB1 gene with Parkinson's disease. Parkinsonism Relat Disord. 15, 422-4.

Weston, C. R., Davis, R. J., 2007. The JNK signal transduction pathway. Curr Opin Cell Biol. 19, 142-9.

Whetton, A. D., Dexter, T. M., 1989. Myeloid haemopoietic growth factors. Biochim Biophys Acta. 989, 111-32.

Whitton, P. S., 2007. Inflammation as a causative factor in the aetiology of Parkinson's disease. Br J Pharmacol. 150, 963-76.

Wilson, E. H., Weninger, W., Hunter, C. A., Trafficking of immune cells in the central nervous system. J Clin Invest. 120, 1368-79. 
Wong, D., Dorovini-Zis, K., 1992. Upregulation of intercellular adhesion molecule-1 (ICAM-1) expression in primary cultures of human brain microvessel endothelial cells by cytokines and lipopolysaccharide. J Neuroimmunol. 39, 11-21.

Wu, D. C., Jackson-Lewis, V., Vila, M., Tieu, K., Teismann, P., Vadseth, C., Choi, D. K., Ischiropoulos, H., Przedborski, S., 2002. Blockade of microglial activation is neuroprotective in the 1-methyl-4-phenyl-1,2,3,6-tetrahydropyridine mouse model of Parkinson disease. J Neurosci. 22, 1763-71.

Wu, D. C., Teismann, P., Tieu, K., Vila, M., Jackson-Lewis, V., Ischiropoulos, H., Przedborski, S., 2003. NADPH oxidase mediates oxidative stress in the 1-methyl4-phenyl-1,2,3,6-tetrahydropyridine model of Parkinson's disease. Proceedings of the National Academy of Sciences of the United States of America. 100, 61456150.

Wu, L., Gu, J., Cui, H., Zhang, Q. Y., Behr, M., Fang, C., Weng, Y., Kluetzman, K., Swiatek, P. J., Yang, W., Kaminsky, L., Ding, X., 2005. Transgenic mice with a hypomorphic NADPH-cytochrome P450 reductase gene: effects on development, reproduction, and microsomal cytochrome P450. J Pharmacol Exp Ther. 312, 3543.

Xia, X. G., Harding, T., Weller, M., Bieneman, A., Uney, J. B., Schulz, J. B., 2001. Gene transfer of the JNK interacting protein-1 protects dopaminergic neurons in the MPTP model of Parkinson's disease. Proc Natl Acad Sci U S A. 98, 10433-8.

Xiao, H., Banks, W. A., Niehoff, M. L., Morley, J. E., 2001. Effect of LPS on the permeability of the blood-brain barrier to insulin. Brain Res. 896, 36-42. 
Yamamori, T., Inanami, O., Sumimoto, H., Akasaki, T., Nagahata, H., Kuwabara, M., 2002. Relationship between $\mathrm{p} 38$ mitogen-activated protein kinase and small GTPase Rac for the activation of NADPH oxidase in bovine neutrophils. Biochem Biophys Res Commun. 293, 1571-8.

Yang, L., Lindholm, K., Konishi, Y., Li, R., Shen, Y., 2002. Target depletion of distinct tumor necrosis factor receptor subtypes reveals hippocampal neuron death and survival through different signal transduction pathways. J Neurosci. 22, 3025-32.

Yang, L., Matthews, R. T., Schulz, J. B., Klockgether, T., Liao, A. W., Martinou, J. C., Penney, J. B., Jr., Hyman, B. T., Beal, M. F., 1998. 1-Methyl-4-phenyl-1,2,3,6tetrahydropyride neurotoxicity is attenuated in mice overexpressing $\mathrm{Bcl}-2$. $\mathrm{J}$ Neurosci. $18,8145-52$.

Yang, W., Chen, L., Ding, Y., Zhuang, X., Kang, U. J., 2007. Paraquat induces dopaminergic dysfunction and proteasome impairment in DJ-1-deficient mice. Hum Mol Genet. 16, 2900-10.

Yang, W., Tiffany-Castiglioni, E., 2008. Paraquat-induced apoptosis in human neuroblastoma SH-SY5Y cells: involvement of p53 and mitochondria. J Toxicol Environ Health A. 71, 289-99.

Yasuhara, T., Shingo, T., Date, I., 2007. Glial cell line-derived neurotrophic factor (GDNF) therapy for Parkinson's disease. Acta Med Okayama. 61, 51-6.

Yoon, S. H., Shim, Y. S., Park, Y. H., Chung, J. K., Nam, J. H., Kim, M. O., Park, H. C., Park, S. R., Min, B. H., Kim, E. Y., Choi, B. H., Park, H., Ha, Y., 2007. Complete spinal cord injury treatment using autologous bone marrow cell transplantation 
and bone marrow stimulation with granulocyte macrophage-colony stimulating factor: Phase I/II clinical trial. Stem Cells. 25, 2066-73.

Zamanian-Daryoush, M., Mogensen, T. H., DiDonato, J. A., Williams, B. R., 2000. NFkappaB activation by double-stranded-RNA-activated protein kinase (PKR) is mediated through NF-kappaB-inducing kinase and IkappaB kinase. Mol Cell Biol. 20, 1278-90.

Zhang, H. T., Li, L. Y., Zou, X. L., Song, X. B., Hu, Y. L., Feng, Z. T., Wang, T. T., 2007. Immunohistochemical distribution of NGF, BDNF, NT-3, and NT-4 in adult rhesus monkey brains. J Histochem Cytochem. 55, 1-19.

Zhang, W., Shin, E. J., Wang, T., Lee, P. H., Pang, H., Wie, M. B., Kim, W. K., Kim, S. J., Huang, W. H., Wang, Y., Hong, J. S., Kim, H. C., 2006. 3-Hydroxymorphinan, a metabolite of dextromethorphan, protects nigrostriatal pathway against MPTPelicited damage both in vivo and in vitro. FASEB J. 20, 2496-511.

Zhang, W., Qin, L., Wang, T., Wei, S. J., Gao, H. M., Liu, J., Zhang, W., Kim, H. C., 2005. 3-hydroxymorphinan is neurotrophic to dopaminergic neurons an is also neuroprotective against LPS-induced neurotoxicity. FASEB J. 19, 395-397.

Zheng, J. S., Tang, L. L., Zheng, S. S., Zhan, R. Y., Zhou, Y. Q., Goudreau, J., Kaufman, D., Chen, A. F., 2005. Delayed gene therapy of glial cell line-derived neurotrophic factor is efficacious in a rat model of Parkinson's disease. Brain Res Mol Brain Res. 134, 155-61.

Zhou, F., Wu, J. Y., Sun, X. L., Yao, H. H., Ding, J. H., Hu, G., 2007. Iptakalim alleviates rotenone-induced degeneration of dopaminergic neurons through 
inhibiting microglia-mediated neuroinflammation. Neuropsychopharmacology. $32,2570-80$.

Zhou, H. F., Liu, X. Y., Niu, D. B., Li, F. Q., He, O. H., Wang, X. M., 2005. Triptolide protects dopaminergic neurons from inflammation-mediated damage induced by lipopolysaccaride intranigral injection. Neurobiol Dis. 18, 441-9.

Zlokovic, B. V., 2008. The blood-brain barrier in health and chronic neurodegenerative disorders. Neuron. 57, 178-201.

Zuccato, C., Cattaneo, E., 2009. Brain-derived neurotrophic factor in neurodegenerative diseases. Nat Rev Neurol. 5, 311-22. 